\title{
Evaluation of Non-Imprinted Polymer Particles for Advanced Treatment of Water and Wastewater
}

\author{
by
}

Audrey Murray

\begin{abstract}
A thesis submitted to the Faculty of Graduate and Postdoctoral Affairs in partial fulfillment of the requirements for the degree of
\end{abstract}

Doctor of Philosophy

in

Environmental Engineering

Department of Civil and Environmental Engineering

Carleton University

Ottawa, Ontario

(C) 2015, Audrey Murray 


\begin{abstract}
This research evaluated the use of non-imprinted polymers (NIP) for treatment of micropollutants and heavy metals. NIP were also compared to powdered activated carbon in competition with humic acid and wastewater. Lastly, conventional separation methods for suspended solids such as centrifugation, filtration, and ballasted sand flocculation were evaluated for removal of NIP particles following treatment.
\end{abstract}

In Chapter 5, NIP were evaluated for removal of EDCs and pharmaceuticals from water and wastewater. NIP were highly effective at removing EDCs from single solute solutions, and were also able to remove EDCs from a $0.5 \mathrm{ppm}$ mixture of five EDCs. They were able to remove 9 out of 12 of the pharmaceuticals to some degree from deionized water and 3 out of 7 of the pharmaceuticals measured in wastewater. Overall, NIP were effective for removal of EDCs, but further study is required to determine whether they can remove pharmaceuticals. In Chapter 6, NIP were shown to be very effective for removal of lead from both river water and wastewater, but less effective for other heavy metal ions. Chapter 7 investigated mechanisms of competition for non-imprinted polymers (NIP) and activated carbon with humic acid and wastewater. Experiments were conducted for single-solute adsorption of methylene blue dye, simultaneous adsorption with humic acid and wastewater, and pre-loading with humic acid and wastewater followed by adsorption of the dye. The only decrease observed was for simultaneous adsorption with humic acid for NIP (for a $90 \%$ confidence limit). Humic acid and wastewater increased adsorption for the activated carbon, Norit PAC 200, for pre-loading with humic acid and simultaneous 
exposure to wastewater. Adsorbed humic acid or NOM from wastewater may have increased the negative surface charge of the activated carbon and increased adsorption, cancelling out decreases due to competition. In Chapter 8, removal of NIP following treatment was investigated using conventional separation methods for suspended solids such as filtration, centrifugation, and ballasted flocculation. Centrifugation was the most effective, followed by filtration, and ballasted flocculation, but all three separation methods effectively removed NIP particles. The results of this thesis showed that NIP have strong potential for water and wastewater treatment. 


\section{TABLE OF CONTENTS}

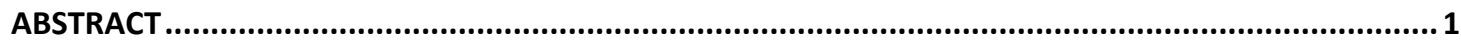

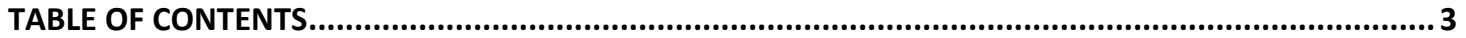

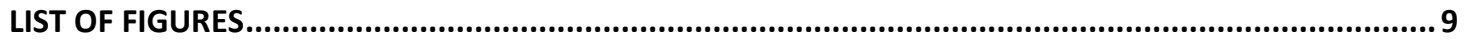

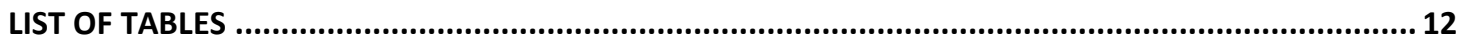

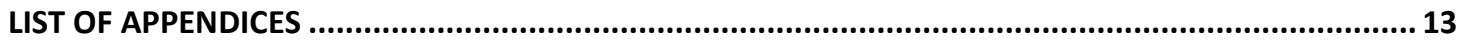

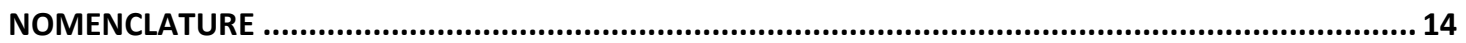

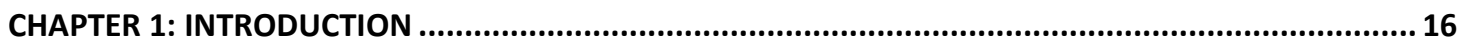

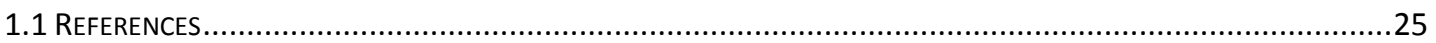

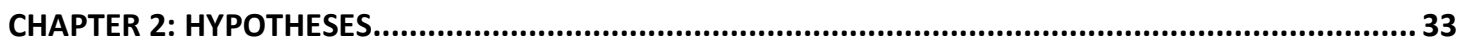

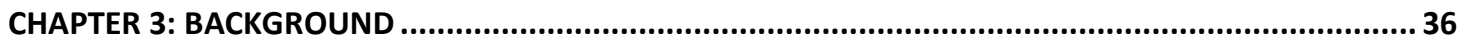

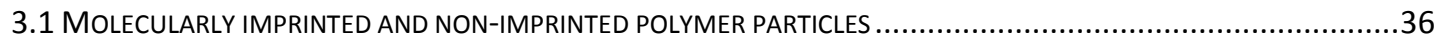

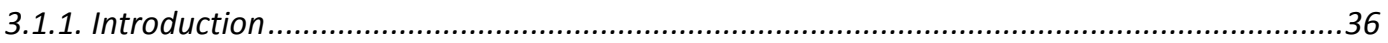

3.1.2 Design of molecularly imprinted and non-imprinted particles .........................................38

3.1.2.1 The covalent approach for design of molecularly imprinted and non-imprinted particles........ 38

3.1.2.2 The non-covalent approach for design of molecularly imprinted and non-imprinted polymers 38

3.1.2.3 The stochiometric non-covalent approach for design of molecularly imprinted and non-

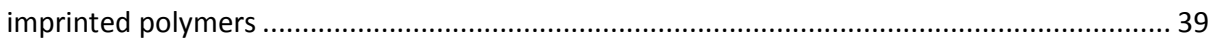

3.1.2.4 The semi-covalent approach for design of molecularly imprinted and non-imprinted polymers

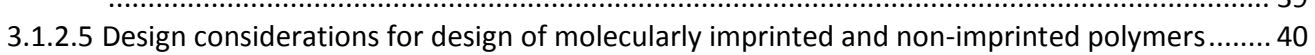

3.1.3 Types of binding and adsorption that may occur on molecularly imprinted and non-

imprinted polymers.

3.1.4 Thermodynamics of specific and non-specific binding for molecularly imprinted and non-

imprinted polymers .................................................................................................45

3.1.5 Molecularly imprinted particles for solid phase pre-concentration for analytical analysis....47

3.1.5.1 Bleeding of the template molecule during analytical pre-concentration and analysis .............. 48

3.2 APPLICATION OF MOLECULARLY IMPRINTED AND NON-IMPRINTED POLYMERS FOR REMOVAL OF EMERGING

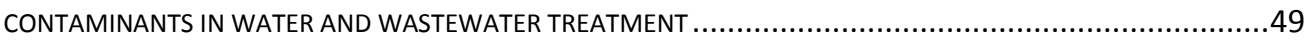

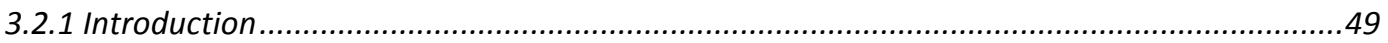

3.2.2 What are molecularly imprinted and non-imprinted polymers? ..........................................52

3.2.3 Potential use of MIP for water and wastewater treatment ............................................53

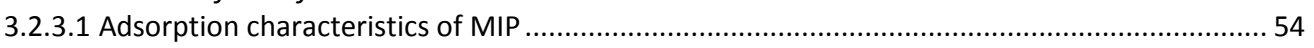

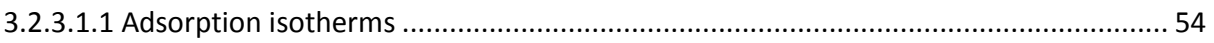

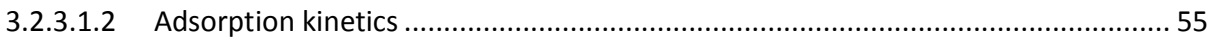

3.2.3.2 Effect of water characteristics on MIP performance..................................................... 56

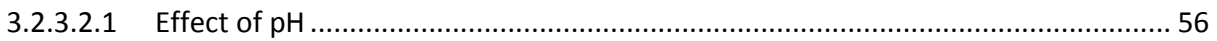

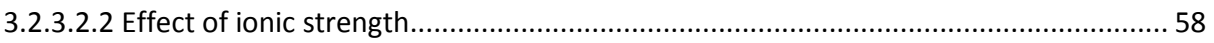

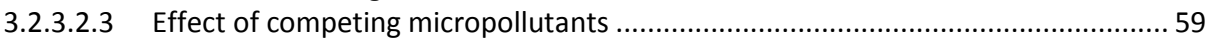

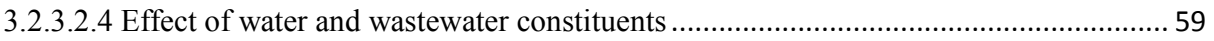

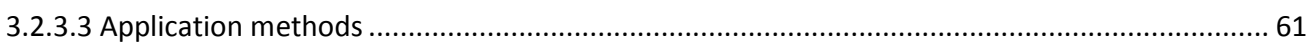

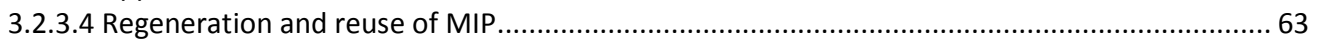

3.2.4 Potential use of NIP for water and wastewater treatment ............................................65

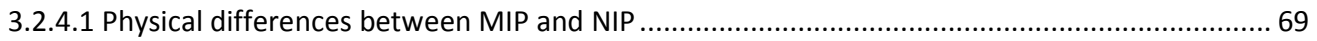

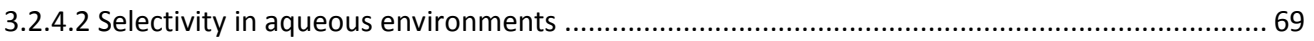

3.2.4.3 Effect of water and wastewater constituents on NIP........................................................ 70

3.2.4.4 Application of NIP for water and wastewater treatment................................................... 72 


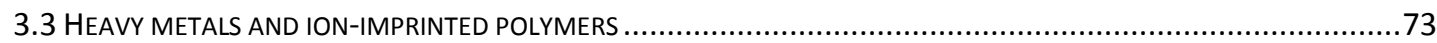

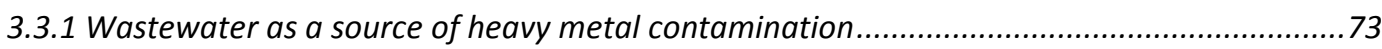

3.3.2 Heavy metal effects on crops and agriculture ................................................................. 74

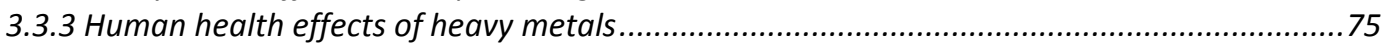

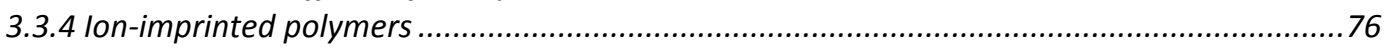

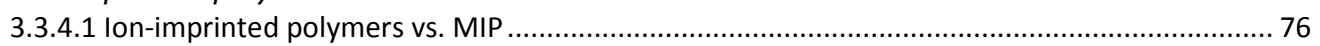

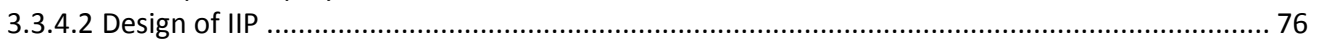

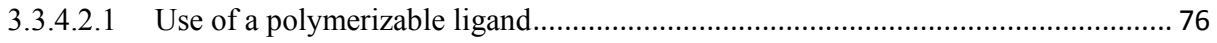

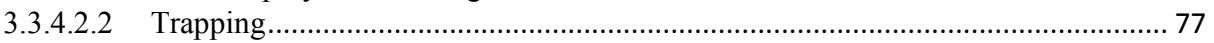

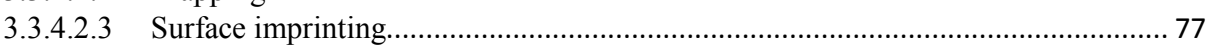

3.3.4.3 Specific and non-specific interactions ............................................................................... 78

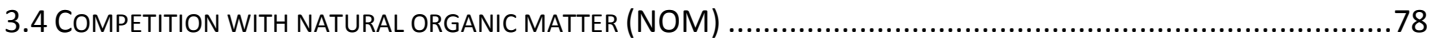

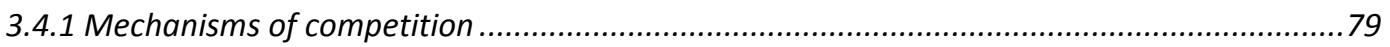

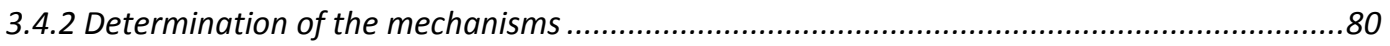

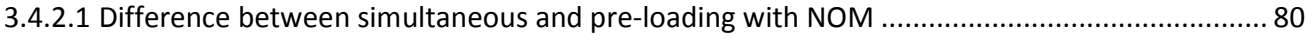

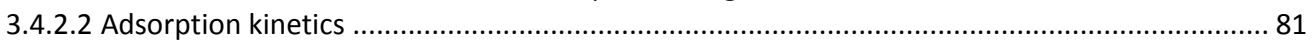

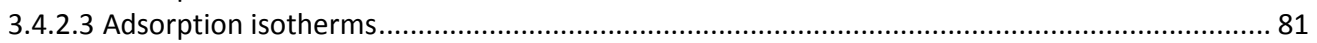

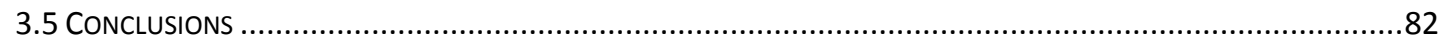

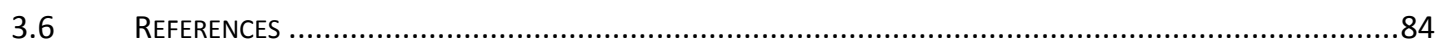

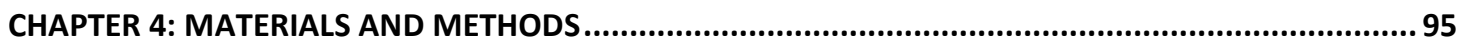

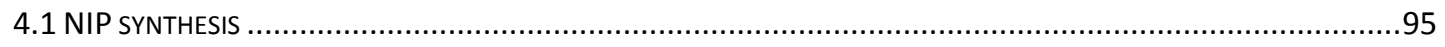

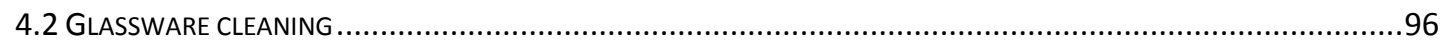

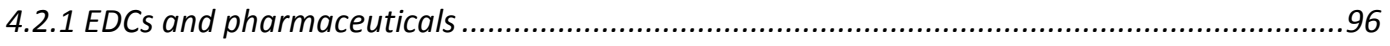

4.2.2 Natural organic matter (NOM) and heavy metals ...........................................................97

4.3 USE OF NON-IMPRINTED POLYMERS FOR REMOVAL OF ENDOCRINE DISRUPTINGCOMPOUNDS AND PHARMACEUTICALS

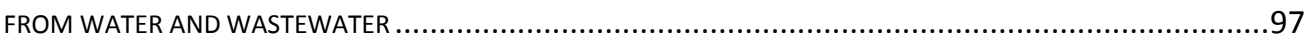

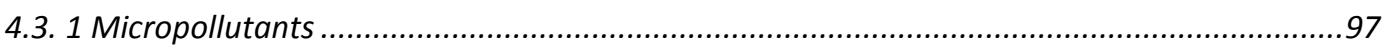

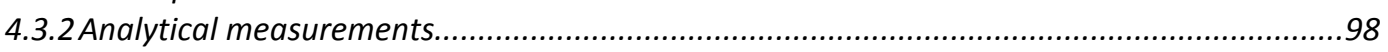

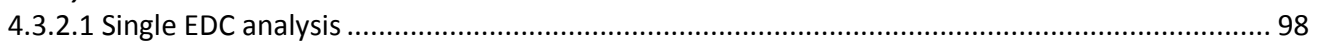

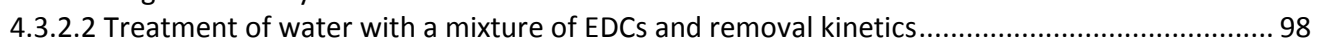

4.3.2.3 Removal of pharmaceuticals .......................................................................................... 100

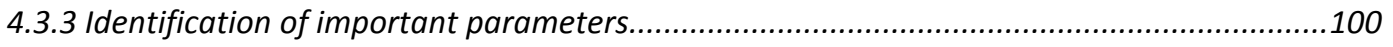

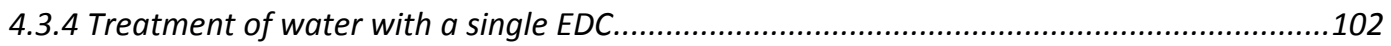

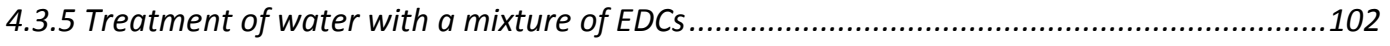

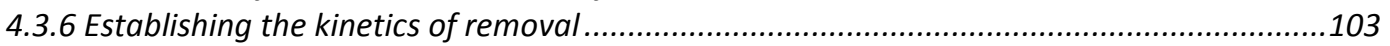

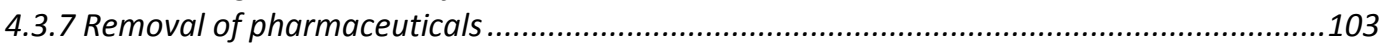

4.4 USE OF NON-IMPRINTED POLYMERS FOR REMOVAL OF HEAVY METAL IONS FROM WATER AND WASTEWATER.........104

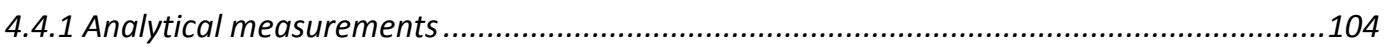

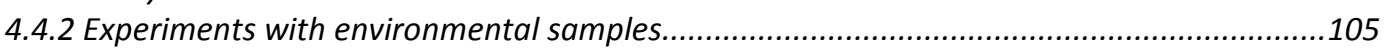

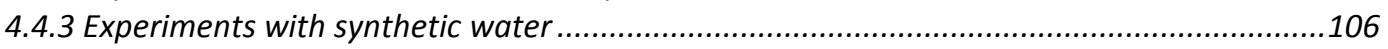

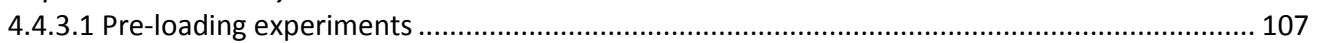

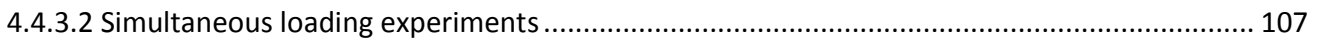

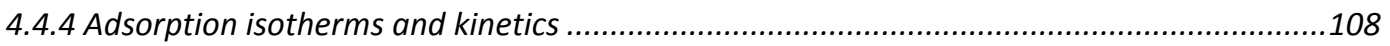

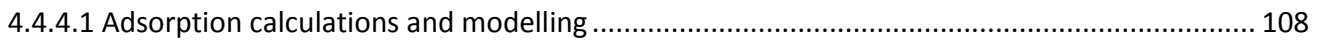

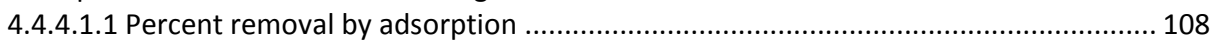

4.4.4.1.2 Percent removal by other mechanisms ............................................................. 108

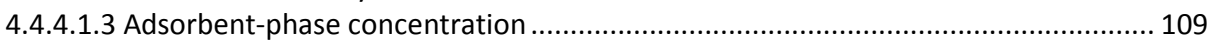

4.4.4.1.4 Langmuir adsorption isotherm ………………………...................................... 110

4.4.4.1.5 Freundlich adsorption isotherm …………………........................................ 111

4.4.5 Adsorption kinetics for adsorption of lead onto NIP ........................................................... 112

4.4.6 Isotherm experiments for adsorption of lead onto NIP ....................................................... 112

4.4.7 Isotherm experiments for adsorption of a mixture of heavy metals onto NIP............................ 112 
4.5 COMPETITIVE EFFECTS OF HUMIC ACID AND WASTEWATER ON ADSORPTION OF METHYLENE BLUE DYE BY ACTIVATED

CARBON AND NON-IMPRINTED POLYMERS ...................................................................... 113

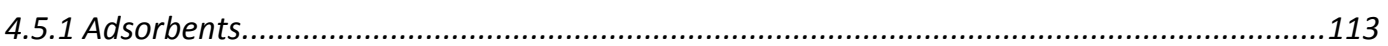

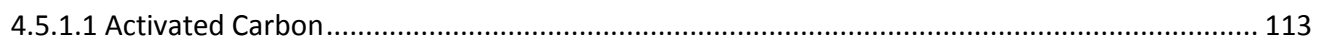

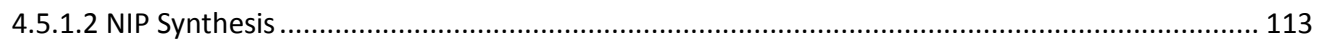

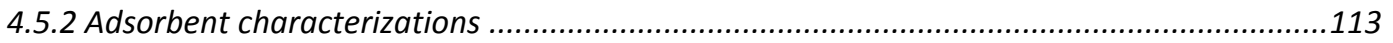

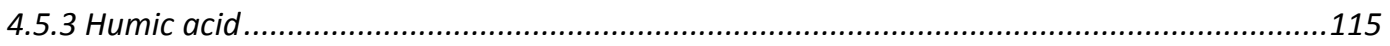

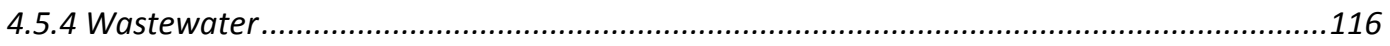

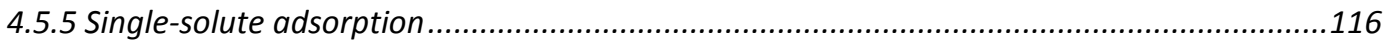

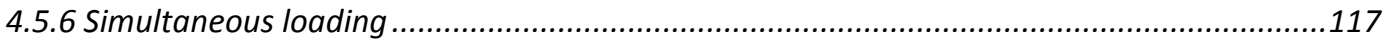

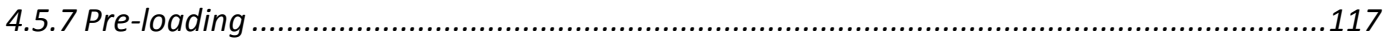

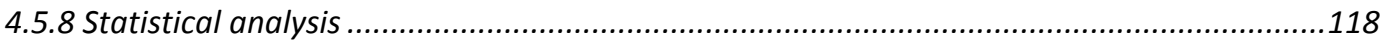

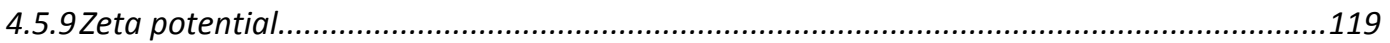

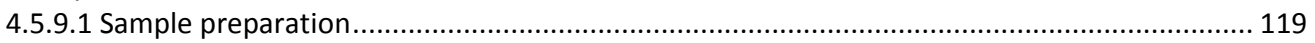

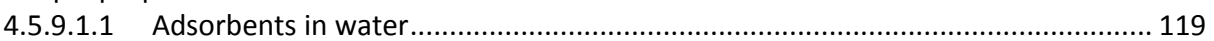

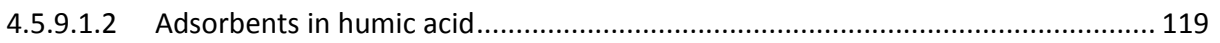

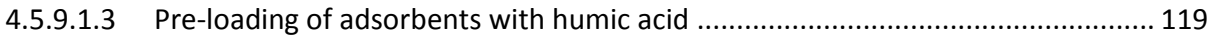

4.5.9.2 Zeta potential measurements and instrument ................................................................ 120

4.6 INVESTIGATION OF PHYSICAL SEPARATION PROCESSES FOR REMOVAL OF NON-IMPRINTED POLYMERS FOLLOWING

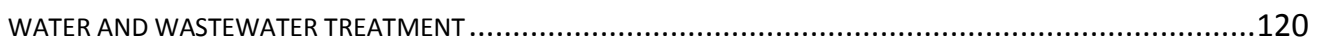

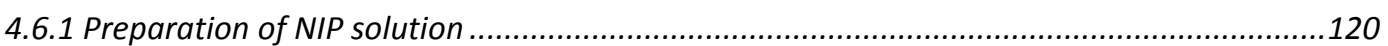

4.6.2 Removal of NIP with gravity settling ............................................................................. 121

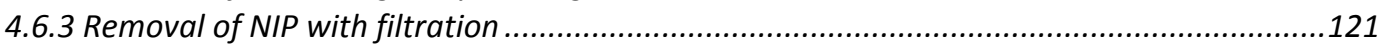

4.6.4 Removal of NIP with centrifugation ................................................................................121

4.6.5 Removal of NIP with ballasted flocculation................................................................. 122

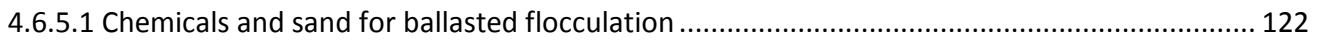

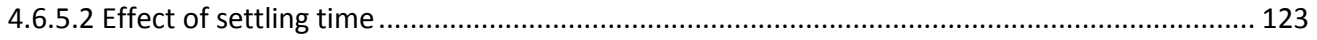

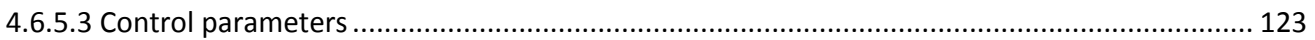

4.6.5.4 Optimization of ballasted flocculation conditions............................................................. 124

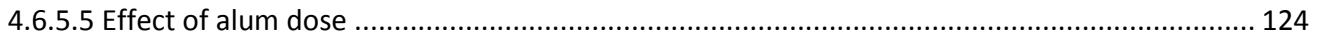

4.6.6 Scanning electron microscope (SEM) images ..............................................................125

4.6.7 Evaluation of removal efficiency with various analytical devices.....................................125

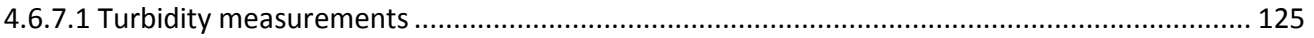

4.6.7.2 Particle size and distribution as measured by the DPA 4100 particle analysis system............. 125

4.6.7.3 Particle size as measured by the Nano ZS Zetasizer .............................................................. 126

4.6.8 Exposure of NIP to acidic and basic conditions.............................................................126

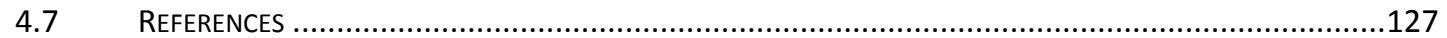

\section{CHAPTER 5: USE OF NON-IMPRINTED POLYMERS FOR REMOVAL OF ENDOCRINE DISRUPTING COMPOUNDS AND PHARMACEUTICALS FROM WATER AND WASTEWATER ...................................... 129}

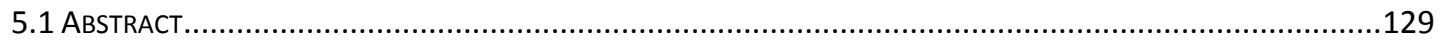

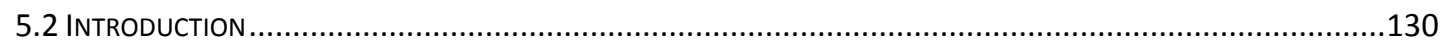

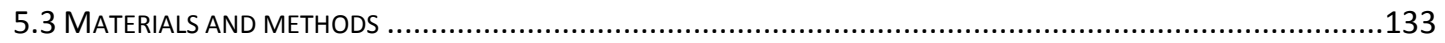

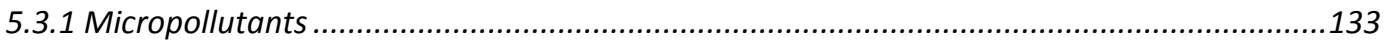

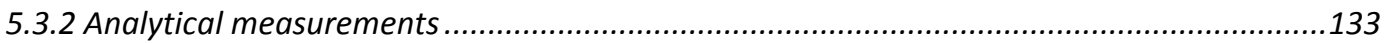

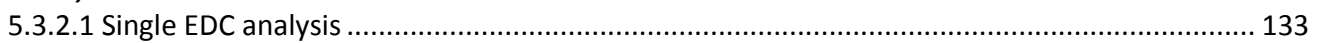

5.3.2.2 Treatment of water with a mixture of EDCs and removal kinetics........................................ 134

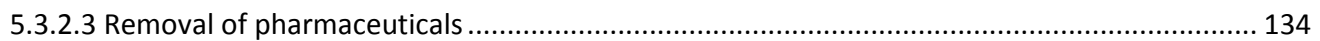

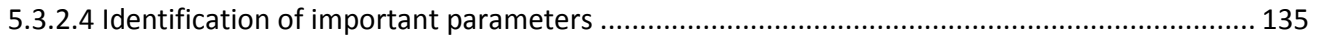

5.3.3 Treatment of water with a single EDC...................................................................... 136

5.3.4 Treatment of water with a mixture of EDCs ..............................................................136

5.3.5 Establishing the kinetics of removal .............................................................................137 


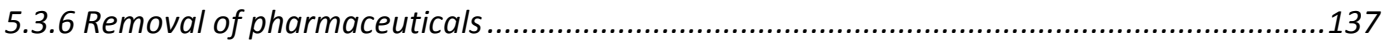

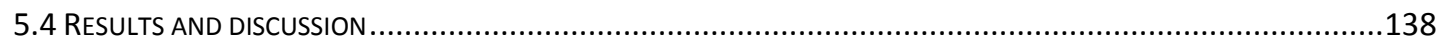

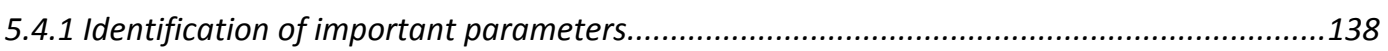

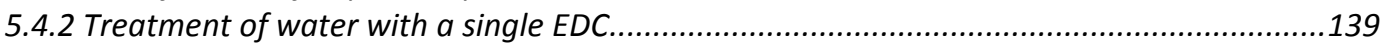

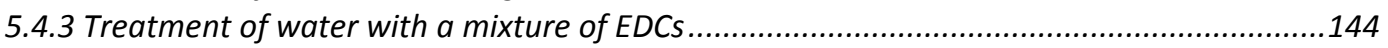

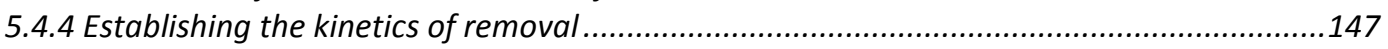

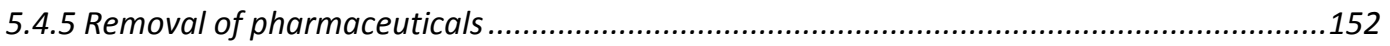

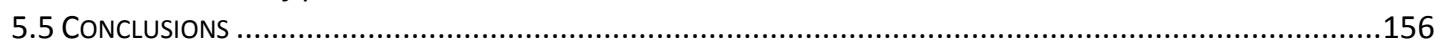

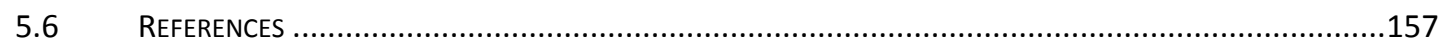

CHAPTER 6: USE OF NON-IMPRINTED POLYMERS FOR REMOVAL OF HEAVY METAL IONS FROM

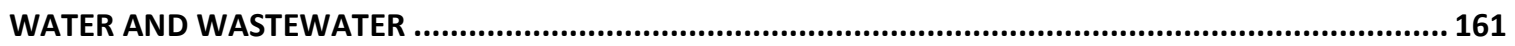

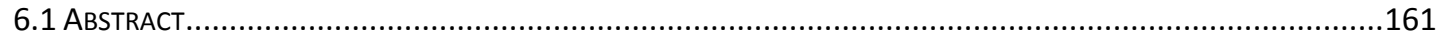

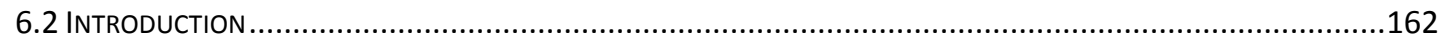

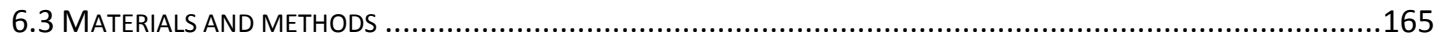

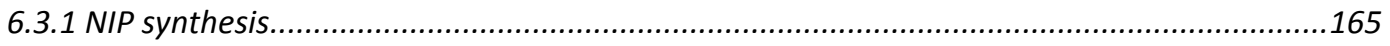

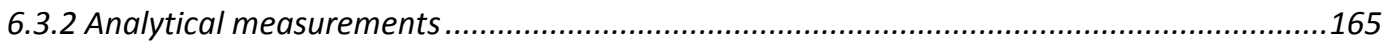

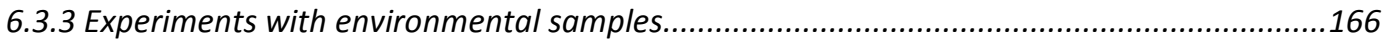

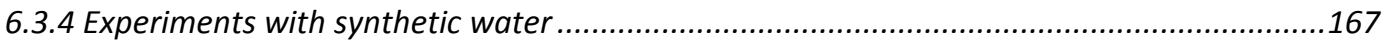

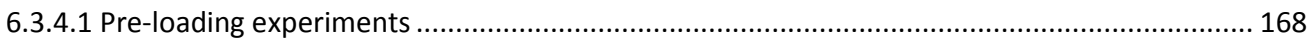

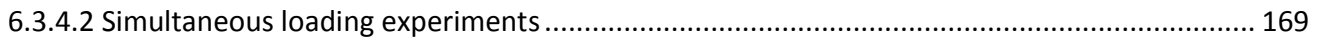

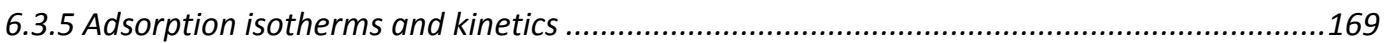

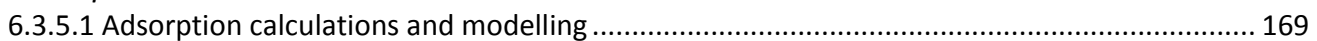

6.3.5.1.1 Percent removal by adsorption........................................................................... 169

6.3.5.1.2 Percent removal by other mechanisms ............................................................... 170

6.3.5.1.3 Adsorbent-phase concentration........................................................................ 170

6.3.5.1.4 Langmuir adsorption isotherm ....................................................................... 171

6.3.5.1.5 Freundlich adsorption isotherm........................................................................ 172

6.3.5.2 Adsorption kinetics for adsorption of lead onto NIP ..................................................... 173

6.3.5.3 Isotherm experiments for adsorption of lead onto NIP .................................................... 174

6.3.5.4 Isotherm experiments for adsorption of a mixture of heavy metals onto NIP........................ 174

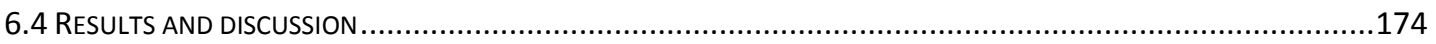

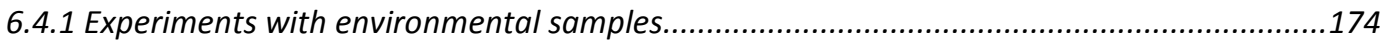

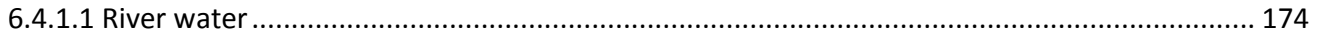

6.4.1.1.1 Water chemistry and adsorption of heavy metal ions ........................................... 179

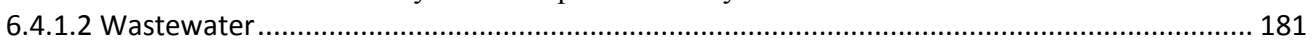

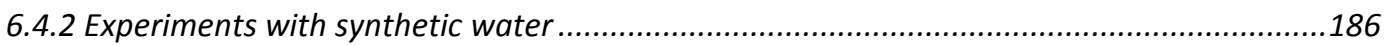

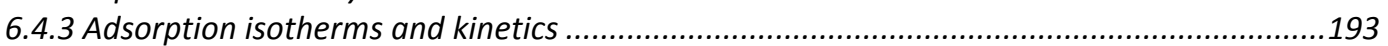

6.4.3.1 Adsorption kinetics for adsorption of a mixture of heavy metals onto NIP ............................ 193

6.4.3.2 Adsorption experiments for adsorption of lead onto NIP ................................................. 195

6.4.3.3 Adsorption experiments for adsorption of a mixture of heavy metals onto NIP .................... 199

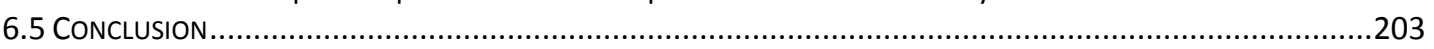

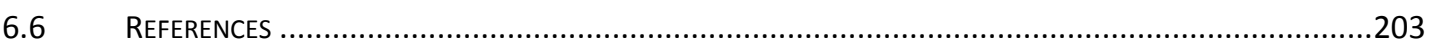

CHAPTER 7: COMPETITIVE EFFECTS OF HUMIC ACID AND WASTEWATER ON ADSORPTION OF METHYLENE BLUE DYE BY ACTIVATED CARBON AND NON-IMPRINTED POLYMERS............................. 208

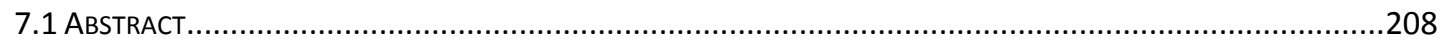

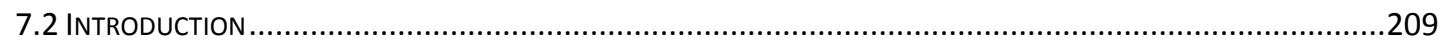

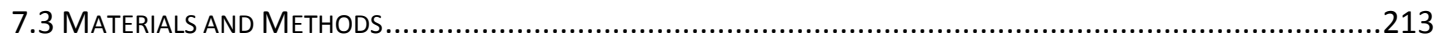

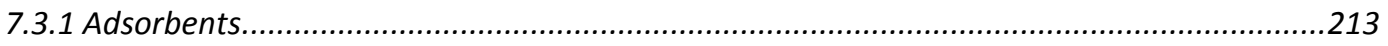

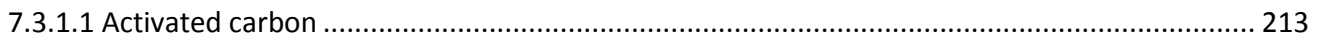

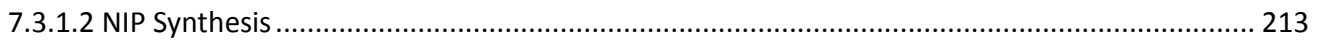




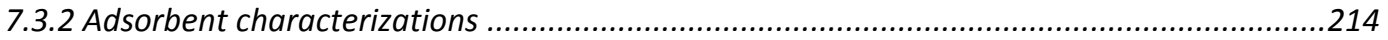

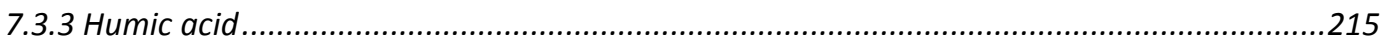

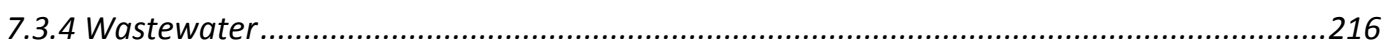

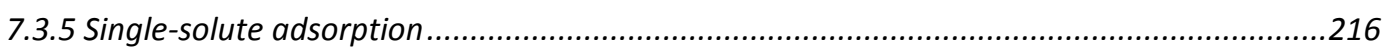

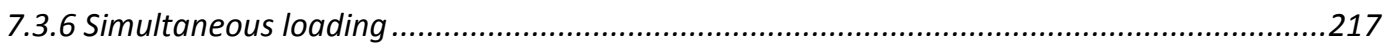

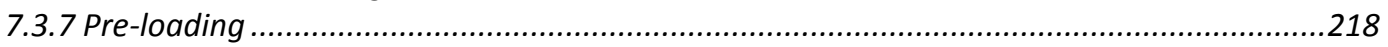

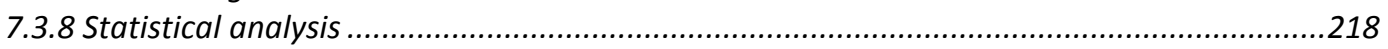

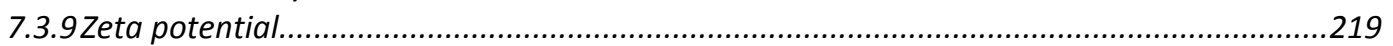

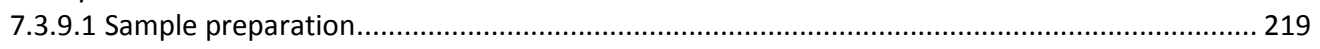

7.3.9.1.1 Adsorbents in water ...................................................................................... 219

7.3.9.1.2 Adsorbents in humic acid............................................................................. 219

7.3.9.1.3 Pre-loading of adsorbents with humic acid ...................................................... 220

7.3.9.1.4 Zeta potential measurements and instrument .................................................. 220

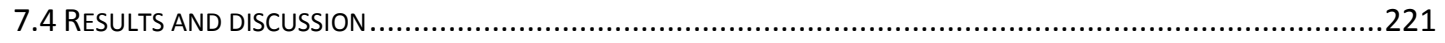

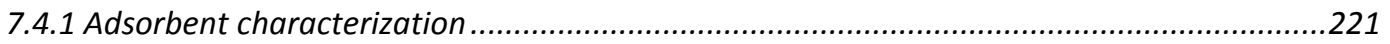

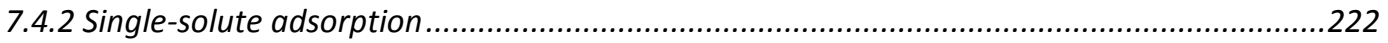

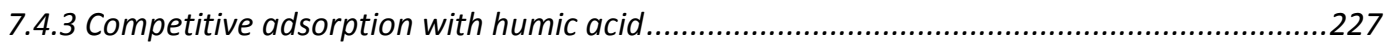

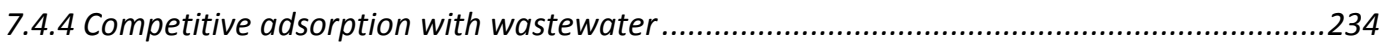

7.4.5 Effect of zeta potential on adsorption performance for NIP and PAC 200 ..........................238

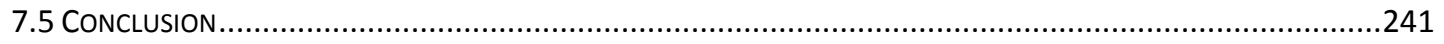

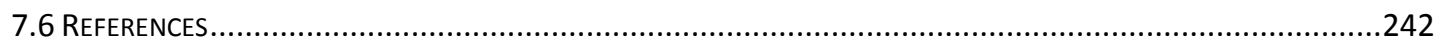

CHAPTER 8: INVESTIGATION OF PHYSICAL SEPARATION PROCESSES FOR REMOVAL OF NONIMPRINTED POLYMERS FOLLOWING WATER AND WASTEWATER TREATMENT .....................................250

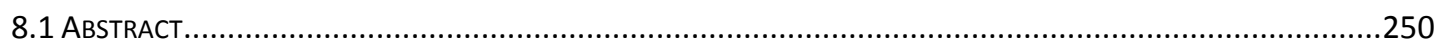

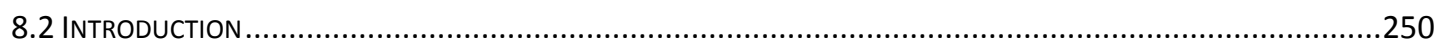

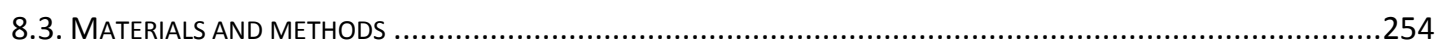

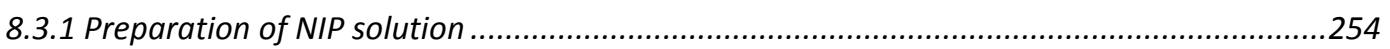

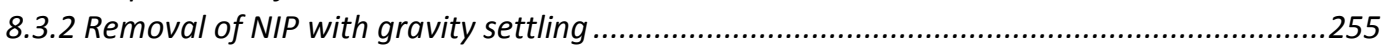

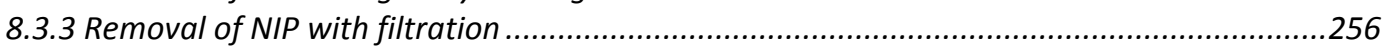

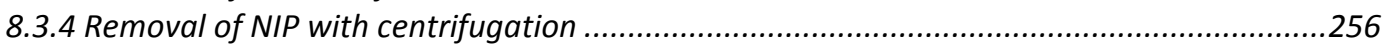

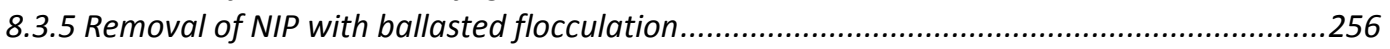

8.3.5.1 Chemicals and sand for ballasted flocculation ................................................................... 257

8.3.5.2 Effect of settling time ................................................................................................. 257

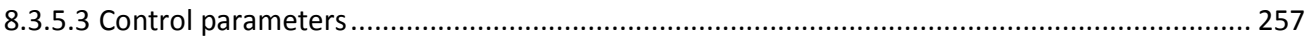

8.3.5.4 Optimization of ballasted flocculation conditions............................................................ 258

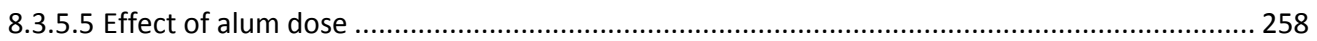

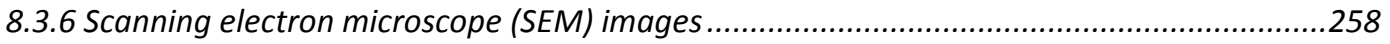

8.3.7 Evaluation of removal efficiency with various analytical devices.......................................259

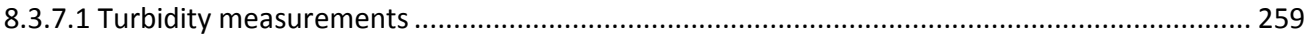

8.3.7.2 Particle size and distribution as measured by the DPA 4100 particle analysis system............. 259

8.3.8 Particle size as measured by the Nano ZS Zetasizer .........................................................259

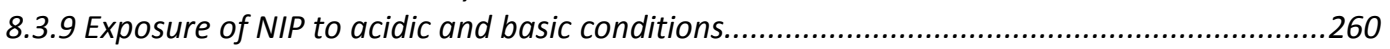

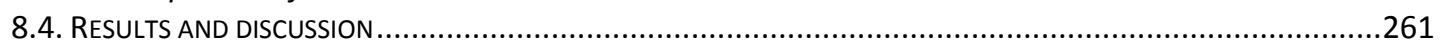

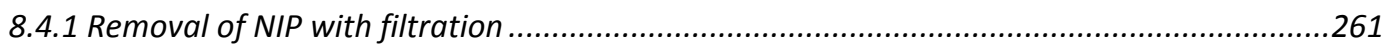

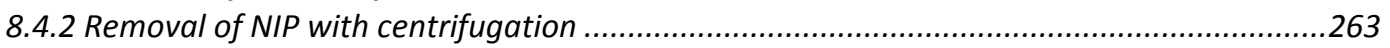

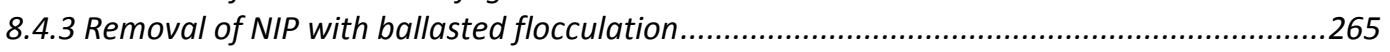

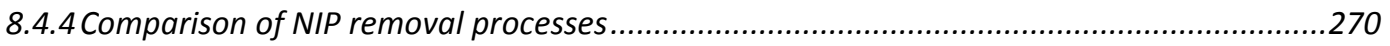

8.4.4.1 Comparison based on turbidity measurements ............................................................... 270

8.4.5 Comparison based on particle counter measurements ...................................................273

8.4.6 Evaluation of removal efficiency with different analytical devices .....................................276

8.4.6.1 Characterization of dry NIP ...................................................................................... 276 


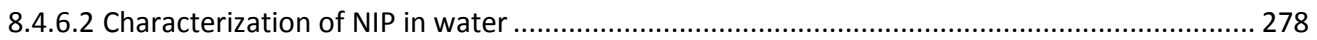

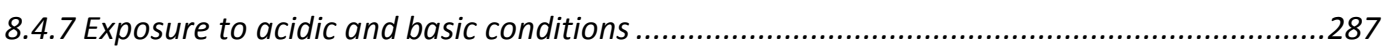

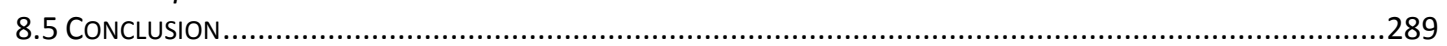

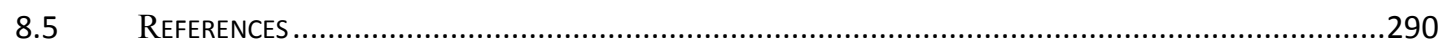

CHAPTER 9: CONCLUSIONS AND FUTURE WORK ..................................................................295

APPENDIX A: REPORTING LIMITS FOR PHARMACEUTICALS................................................ 301

APPENDIX B: WATER SAMPLE CHARACTERISTICS ............................................................. 302

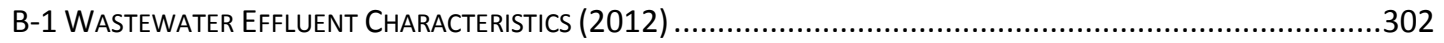

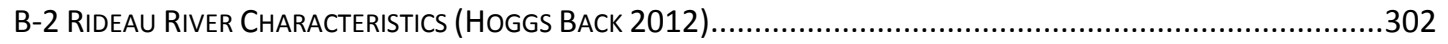

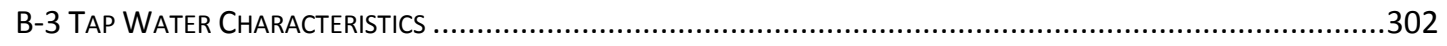

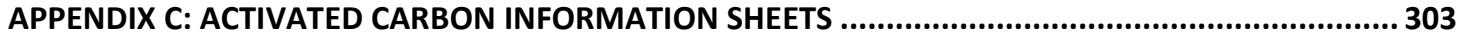

APPENDIX D: SUWANNEE RIVER HUMIC ACID STANDARD (II) DATA PROVIDED BY THE

INTERNATIONAL HUMIC SUBSTANCES SOCIETY .........................................................................306

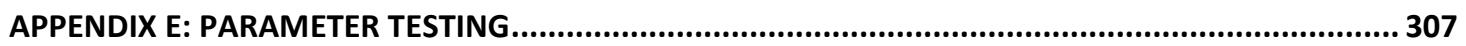

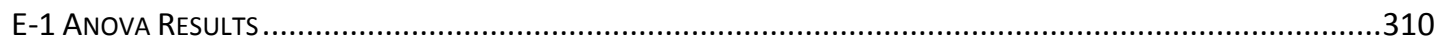

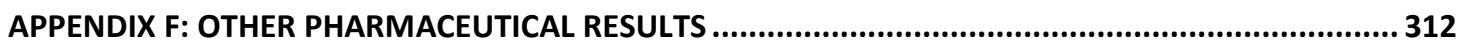

APPENDIX G: DISTRIBUTION OF HYDROLYSIS PRODUCTS FOR LEAD, COPPER, ZINC, AND NICKEL

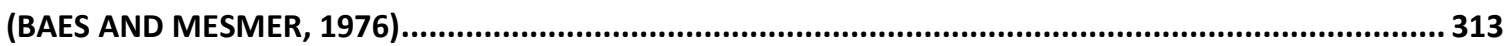

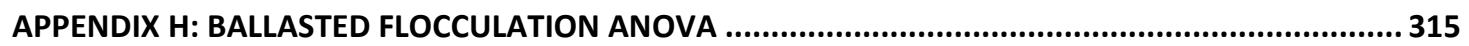




\section{LIST OF FIGURES}

FIGURE 3-1: SCHEMATIC SHOWING THE FORMATION, DESIGN, AND USE OF MIP PARTICLES (ALEXANDER, ET AL., 2006)

FIGURE 3-2: SPECIFIC, SEMI-SPECIFIC AND NON-SPECIFIC ADSORPTION OF ENDOCRINE DISRUPTORS TO MIP PREPARED WITH AN E2 TEMPLATE (ZHANG \& HU, 2008)........................ 43

FIGURE5-1: REMOVAL OF E2, EE2, AND BPA (2-14 PPB) WITH NIP.......................................... 141

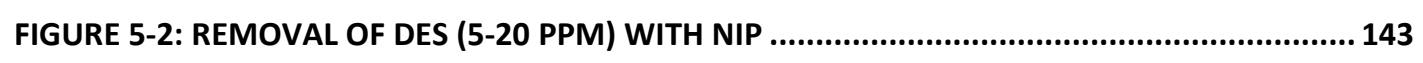

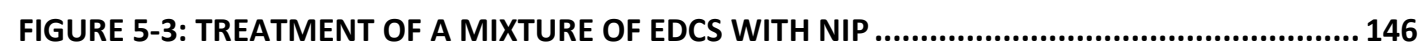

FIGURE 5-4 (A): KINETICS OF REMOVAL FOR A MIXTURE OF 5 EDCS WITH 50 MG/L OF NIP OVER A

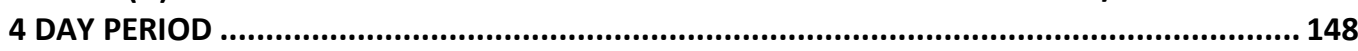

FIGURE 5-4 (B): KINETICS OF REMOVAL FOR A MIXTURE OF 5 EDCS WITH 500 MG/L OF NIP OVER

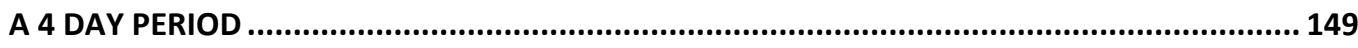

FIGURE 5-5: TREATMENT OF PHARMACEUTICALS IN DEIONIZED WATER WITH NIP..................... 154

FIGURE 5-6: TREATMENT OF PHARMACEUTICALS IN WASTEWATER WITH NIP.......................... 155

FIGURE 6-1 (A): PERCENT REMOVED VIA MECHANISMS OTHER THAN ADSORPTION BY NIP VERSUS INITIAL HEAVY METAL CONCENTRATION FOR LEAD, COPPER, ZINC, AND NICKEL IN RIVER WATER

FIGURE 6-1 (B): PERCENT REMOVAL BY NIP VERSUS INITIAL HEAVY METAL CONCENTRATION FOR LEAD, COPPER, ZINC, AND NICKEL IN RIVER WATER 176

FIGURE 6-1 (C): AMOUNT ADSORBED BY NIP VERSUS INITIAL HEAVY METAL CONCENTRATION FOR LEAD, COPPER, ZINC, AND NICKEL IN RIVER WATER.

FIGURE 6-2 (A): PERCENT REMOVAL VIA MECHANISMS OTHER THAN ADSORPTION BY NIP VERSUS INITIAL HEAVY METAL CONCENTRATION FOR LEAD, COPPER, ZINC AND NICKEL IN WASTEWATER.

FIGURE 6-2 (B) PERCENT REMOVAL BY NIP VERSUS INITIAL HEAVY METAL CONCENTRATION FOR LEAD, COPPER, ZINC, AND NICKEL IN WASTEWATER.

FIGURE 6-2 (C): AMOUNT ADSORBED BY NIP VERSUS INITIAL HEAVY METAL CONCENTRATION FOR LEAD, COPPER, ZINC AND NICKEL IN WASTEWATER.

FIGURE 6-3 (A): ADSORPTION OF LEAD, COPPER, ZINC, AND NICKEL ON NIP PRE-LOADED WITH HUMIC ACID.

FIGURE 6-3 (B): ADSORPTION OF LEAD, COPPER, ZINC, AND NICKEL ON NIP SIMULTANEOUSLY LOADED WITH HUMIC ACID

FIGURE 6-3 (C): PERCENT REMOVED VIA MECHANISMS OTHER THAN ADSORPTION BY NIP PARTICLES OR ACTIVATED CARBON VERSUS INITIAL HEAVY METAL CONCENTRATION FOR LEAD, COPPER, ZINC AND NICKEL IN WASTEWATER

FIGURE 6-4 (A): ADSORPTION OF LEAD, COPPER, ZINC, AND NICKEL ON ACTIVATED CARBON PRELOADED WITH HUMIC ACID

FIGURE 6-4 (B): ADSORPTION OF LEAD, COPPER, ZINC AND NICKEL ON ACTIVATED CARBON SIMULTANEOUSLY LOADED WITH HUMIC ACID 192

FIGURE 6-5: KINETICS FOR ADSORPTION OF LEAD FROM DEIONIZED WATER (PH 4) 195 
FIGURE 6-6 (A): LEAD ISOTHERM FOR ADSORPTION ONTO NIP IN DEIONIZED WATER (PH 4).... 197

FIGURE 6-6 (B): LINEARIZED FREUNDLICH MODEL FOR ADSORPTION OF LEAD FROM DEIONIZED WATER ONTO NIP (PH 4)

FIGURE 6-6 (C): LINEARIZED LANGMUIR MODEL FOR ADSORPTION OF LEAD FROM DEIONIZED WATER ONTO NIP (PH 4).

FIGURE 6-7 (A): LEAD ISOTHERM IN DEIONIZED WATER FOR ADSORPTION OF LEAD ONTO NIP WITH COMPETITION FROM COPPER, ZINC, AND NICKEL (PH 4) 200

FIGURE 6-7 (B): LINEARIZED FREUNDLICH MODEL FOR ADSORPTION OF LEAD ONTO NIP WITH COMPETITION FROM COPPER, ZINC, AND NICKEL IN DEIONIZED WATER (PH 4) .................... 200

FIGURE 6-7 (C): LINEARIZED LANGMUIR MODEL FOR ADSORPTION OF LEAD ONTO NIP WITH COMPETITION FROM COPPER, ZINC, AND NICKEL IN DEIONIZED WATER (PH 4) .................... 201

FIGURE 6-8 (A): ADSORPTION ISOTHERMS ADSORPTION OF A MIXTURE OF LEAD, COPPER, ZINC, AND NICKEL ONTO NIP IN DEIONIZED WATER (PH 4) 202

FIGURE 6-8 (B): PERCENT REMOVAL VERSUS NIP CONCENTRATION FOR A MIXTURE OF LEAD, COPPER, ZINC, AND NICKEL IN DEIONIZED WATER (PH 4) 202

FIGURE 7-1: ADSORPTION CAPACITIES OF PAC 200, DARCO S-51, DARCO KB-M, AND NIP FOR VARYING INITIAL MB CONCENTRATIONS IN DEIONIZED WATER 224

FIGURE 7-2 (A): COMPARISON BETWEEN SINGLE-SOLUTE ADSORPTION OF MB AND SIMULTANEOUS ADSORPTION AND PRE-LOADING WITH HUMIC ACID (20MG/L) FOR NIP .. 229

FIGURE 7-2 (B): COMPARISON BETWEEN SINGLE-SOLUTE ADSORPTION OF MB AND SIMULTANEOUS ADSORPTION AND PRE-LOADING WITH HUMIC ACID (20 MG/L) FOR PAC 200

FIGURE 7-3 (A): COMPARISON BETWEEN SINGLE-SOLUTE ADSORPTION OF MB AND SIMULTANEOUS ADSORPTION AND PRE-LOADING WITH WASTEWATER FOR NIP .235

FIGURE 7-3 (B): COMPARISON BETWEEN SINGLE-SOLUTE ADSORPTION OF MB AND SIMULTANEOUS ADSORPTION AND PRE-LOADING WITH WASTEWATER FOR PAC 200........ 236

FIGURE 7-4: EFFECT OF HUMIC ACID ON THE ZETA POTENTIAL OF NIP AND PAC $200 \ldots . . . . . . . . . . . . .239$

FIGURE 8-1: PERCENT REMOVAL OF NIP WITH FILTRATION BASED ON TURBIDITY MEASUREMENTS

FIGURE 8-2 (A): PERCENT REMOVAL OF NIP BY CENTRIFUGATION AT VARYING CENTRIFUGE SPEEDS FOR 3 MINUTES BASED ON TURBIDITY MEASUREMENTS

FIGURE 8-2 (B): PERCENT REMOVAL OF NIP BY CENTRIFUGATION AT 10,000 RPM FOR VARYING CENTRIFUGE TIMES BASED ON TURBIDITY MEASUREMENTS

FIGURE 8-3 (A): PERCENT REMOVAL OF NIP BY GRAVITY SETTLING FOR EXTENDED SETTLING TIMES BASED ON TURBIDITY MEASUREMENTS.

FIGURE 8-3 (B): PERCENT REMOVAL OF NIP BY BALLASTED FLOCCULATION WITH EXTENDED SETTLING TIMES BASED ON TURBIDITY MEASUREMENTS

FIGURE 8-4(A):PERCENT REMOVAL OF NIP IN TAP WATER BY BALLASTED FLOCCULATION FOR VARYING ALUM DOSES BASED ON TURBIDITY MEASUREMENTS 269

FIGURE 8-4 (B): PERCENT REMOVAL OF NIP IN WASTEWATER BY BALLASTED FLOCCULATION FOR VARYING ALUM DOSES BASED ON TURBIDITY MEASUREMENTS 269 
FIGURE 8-5: COMPARISON OF PERCENT REMOVAL OF NIP FOR VARIOUS REMOVAL METHODS BASED ON TURBIDITY MEASUREMENTS.................................................................2 271

FIGURE 8-6(A): PARTICLE COUNT CONCENTRATIONS MEASURED BY THE DPA 4100 PARTICLE ANALYSIS SYSTEM BEFORE AND AFTER NIP REMOVAL FOR VARIOUS REMOVAL METHODS 274

FIGURE 8-6 (B): PERCENT REMOVAL OF NIP FOLLOWING REMOVAL WITH VARIOUS REMOVAL METHODS BASED ON PARTICLE CONCENTRATIONS MEASURED BY THE DPA 4100 PARTICLE ANALYSIS SYSTEM.

FIGURE 8-7: SEM IMAGE OF NIP PARTICLES 277

FIGURE 8-8 (A): NIP PARTICLE SIZE DISTRIBUTION IN WATER AS MEASURED BY THE NANO ZS ZETASIZER

FIGURE 8-8 (B): NIP PARTICLE SIZE DISTRIBUTION IN WATER AS MEASURED BY THE DPA 4100 PARTICLE ANALYSIS SYSTEM

FIGURE 8-9 (A): NIP PARTICLE SIZE DISTRIBUTION FOLLOWING CENTRIFUGE TREATMENT AS MEASURED BY THE NANO ZS ZETASIZER (2 REPLICATES).

FIGURE 8-9 (B): NIP PARTICLE SIZE DISTRIBUTION FOLLOWING CENTRIFUGE TREATMENT AS MEASURED BY THE DPA 4100 PARTICLE ANALYSIS SYSTEM

FIGURE 8-10 (A): NIP PARTICLE SIZE DISTRIBUTION FOLLOWING FILTRATION AS MEASURED BY THE NANO ZS ZETASIZER (2 REPLICATES)

FIGURE 8-10 (B): NIP PARTICLE SIZE DISTRIBUTION FOLLOWING FILTRATION AS MEASURED BY THE DPA 4100 PARTICLE ANALYSIS SYSTEM 285

FIGURE 8-11 (A): NIP PARTICLE SIZE DISTRIBUTION FOLLOWING BALLASTED FLOCCULATION AS MEASURED BY THE NANO ZS ZETASIZER (2 REPLICATES).

FIGURE 8-11(B): NIP PARTICLE SIZE DISTRIBUTION FOLLOWING BALLASTED FLOCCULATION AS MEASURED BY THE DPA 4100 PARTICLE ANALYSIS SYSTEM .

FIGURE 8-12: AVERAGE NIP PARTICLE SIZES BEFORE AND AFTER VARIOUS TREATMENTS AS MEASURED BY THE NANO ZS ZETASIZER

FIGURE 8-13 (A): SEM IMAGE OF NIP PARTICLES FOLLOWING 24 H EXPOSURE TO PH 2 (ACID) . 288 FIGURE 8-13 (B): SEM IMAGE OF NIP PARTICLES FOLLOWING 24 H EXPOSURE TO PH 11 (BASE) 289 


\section{LIST OF TABLES}

TABLE 3-1: BINDING EFFICIENCIES FOR MIP AND NIP PREPARED WITH VARIOUS METHODS ......................................67

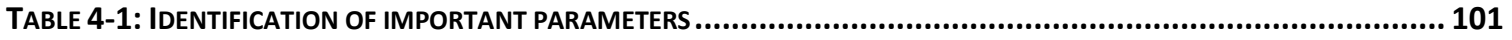

TABLE 4-2: CHEMICAL PROPERTIES AND FUNCTIONAL GROUPS FOR SUWANEE RIVER II HUMIC ACID .......................... 115

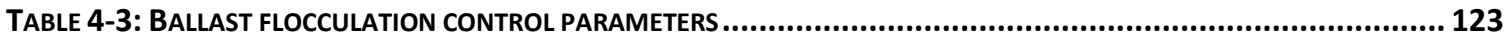




\section{LIST OF APPENDICES}

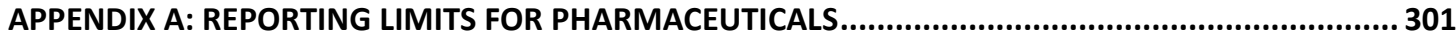

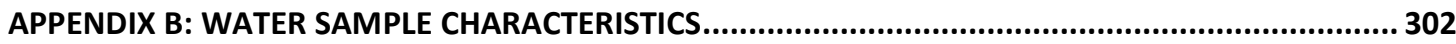

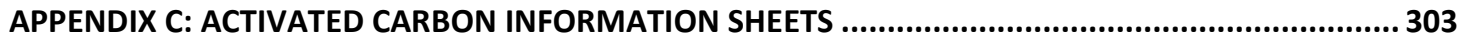

APPENDIX D: SUWANNEE RIVER HUMIC ACID STANDARD (II) DATA PROVIDED BY THE

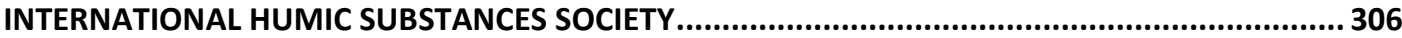

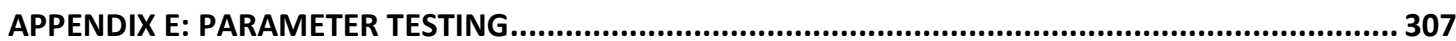

APPENDIX F: OTHER PHARMACEUTICAL RESULTS ........................................................... 312

APPENDIX G: DISTRIBUTION OF HYDROLYSIS PRODUCTS FOR LEAD, COPPER, ZINC, AND NICKEL

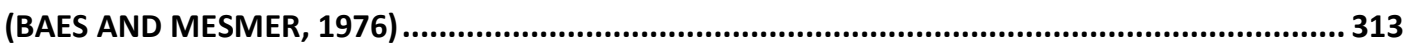

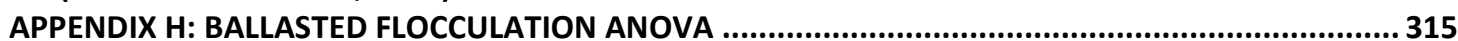




\section{NOMENCLATURE}

\begin{tabular}{ll}
\hline $1-M A-3 M I-B r$ & $1-(\alpha$-methyl acrylate)- 3 methylimidazolium bromide \\
\hline $2,4-D$ & Dichlorophenoxyacetic acid \\
\hline $4-V P$ & 4 - vinyl pyridine \\
\hline AA & Acrylic acid \\
\hline AC & Activated carbon \\
\hline ACN & Acetonitrile \\
\hline ACVA & 2,2 -azobis(4-cyanovaleric acid) \\
\hline AIBN & Azobisisobutyronitrile \\
\hline AIBN & 2,2 - azobisiso butyronitrile \\
\hline AM & Acrylamide \\
\hline BET & Brunauer-Emmett-Teller \\
\hline BJA & Barret-Joyner-Halenda \\
\hline BPA & Bisphenol A \\
\hline CBOD & Carbonaceous biochemical oxygen demand \\
\hline COD & Chemical oxygen demand \\
\hline CFU & Colony forming units \\
\hline DEAEM & dimethylaminoethyl methacrylate \\
\hline DES & Diethylstilbestrol \\
\hline DMF & Dimethyl formamide \\
\hline DVB & Divinyl benzene \\
\hline E1 & Estrone \\
\hline E2 & $17 \beta$ estradiol \\
\hline ECH & Epichlorohydrin \\
\hline EDCs & Endocrine disrupting compounds \\
\hline EE2 & $17 \alpha$ ethinylestradiol \\
\hline EGDMA & Ethylene glycol dimethacrylate \\
\hline FD & Fluorescence detection \\
\hline FFF & Flow field flow fractionation \\
\hline HPLC & High performance liquid chromatography \\
\hline IA & Iticonic acid \\
\hline IIP & Ion-imprinted polymers \\
\hline LC/MS/MS & $\begin{array}{l}\text { High performance liquid chromatography tandem mass } \\
\text { spectrometry }\end{array}$ \\
\hline MAA & Methacrylic acid \\
\hline MB & Methylene blue \\
\hline MeCN & Anhydrous acetonitrile \\
\hline MeOH & Methanol \\
\hline & Molecularly imprinted polymers \\
\hline & \\
\hline MIP & \\
\hline
\end{tabular}




\begin{tabular}{ll}
\hline$M^{n+}$ & Heavy metal with a charge of $n+$ \\
\hline MTBE & Methyl-tert-butylether \\
\hline NF & Nanofiltration \\
\hline NIP & Non-imprinted polymers \\
\hline NOM & Natural organic matter \\
\hline NP & 4-nonylphenol \\
\hline NP & Nonylphenol \\
\hline PAC & Powder activated carbon \\
\hline PAH & Polyaromatic hydrocarbon \\
\hline PDA & Photo diode array detector \\
\hline PFOS & perfluorooctane sulfonate \\
\hline PFP & Pentafluorophenyl \\
\hline ppb & Parts per billion \\
\hline ppt & Parts per trillion \\
\hline PVA & Polyvinyl alcohol \\
\hline QSDFT & Quenched solid density functional theory \\
\hline RF & Radio frequency \\
\hline RO & Reverse osmosis \\
\hline rpm & Revolutions per minute \\
\hline SEC & Size exclusion chromatography \\
\hline SEM & Scanning electron microscope \\
\hline SPE & Solid phase extraction \\
\hline TCE & Trichloroethylene \\
\hline TDS & Total disolved solids \\
\hline TNT & Trinitrotoluene \\
\hline TOC & Total organic carbon \\
\hline TP & Total phosphorus \\
\hline TRIA & Trimethylol propane triacrylate \\
\hline TRIM & Trimethacrylate \\
\hline TSS & Total suspended solids \\
\hline UVC & Ultraviolet C \\
\hline UV-UCGN & UV scanning ultracentrifugation \\
\hline UV-vis & Ultraviolet-visible \\
\hline VP & 4-vinylpyridine \\
\hline & \\
\hline
\end{tabular}




\section{CHAPTER 1: INTRODUCTION}

Endocrine disrupting compounds (EDCs) and pharmaceuticals are typically present at concentrations of low parts per billion ( $\mathrm{ppb}$ ), but are capable of causing adverse health effects at these very low concentrations (Kumar and Xagoraraki, 2010). EDCs have been shown to cause reproductive, immune, hormonal, and neurobehavioural disorders (Yang et al., 2006) as well as breast, testicular, and prostate cancers (Gillesby and Zacharewski, 1998; Ashby et al., 1997). However, effects of long-term exposure to low levels of pharmaceuticals or mixtures of pharmaceuticals remain unknown (World Health Organization, 2012). Heavy metals are capable of accumulating and affecting the function of the heart, brain, kidneys, bone, liver, and other vital organs or displacing essential nutrients required by the body (Singh et al., 2011).

Molecularly imprinted polymers (MIP) and ion imprinted polymers (IIP) are polymers formed in the presence of a template molecule (MIP) or ion (IIP), following polymerization, the template is removed, leaving behind a cavity specific to the template (Masqué, 2001; Alexander, 2006). Non-imprinted polymers (NIP) are prepared in the same way, but without the presence of the template, and thus do not contain template-specific cavities. NIP are still capable of adsorbing template molecules or ions, but adsorption is non-specific (Pichon and Chapuis-Hugon, 2008).

Molecularly imprinted polymers (MIP) and ion imprinted polymers (IIP) were originally designed for solid phase extraction purposes (Masqué, 2001), but have since been applied to water and wastewater treatment (Meng et al., 2005; Zhongbo and Hu, 2008; Hajizadeh 
et al., 2010; Deng et al., 2009; Luo et al., 2011; Guo et al., 2011; Li et al., 2009; Yu et al., 2008; Lin et al., 2008; Krupadam et al., 2010; Le Noir, Lepeuple, et al., 2007; Le Noir, Plieva, et al., 2007; Fernández-Alvarez et al., 2009; Randhawa et al., 2007; Xie et al., 2011; Murray et al., 2011). They have the capability to specifically target one or more compounds of interest despite competition. However, NIP have also been shown to have potential for water and wastewater treatment, and have the advantage of being able to treat a wide range of molecules and ions simultaneously at a lower cost than MIP and IIP (Murray et al., 2011; Murray and Ormeci, 2012).

NIP function through non-specific adsorption, and do not have a specific capacity for one molecule or ion. Because they are non-specific, they have the advantage of being able to treat a wide range of water and wastewater contaminants, but they have the disadvantage of competition from non-trace contaminants such as natural organic matter (NOM). However, Xie et al (2011) found that NOM in river water and lake water interfered with adsorption of bisphenol A less for NIP than activated carbon (Xie et al., 2011). Therefore, it is possible that NIP have the advantage of being able to treat a wide range of contaminants, like activated carbon, while being less affected by competition with NOM. This might be due to the chemical characteristics of the NIP (Lin et al., 2008), or the structure and distribution of their pores (Newcombe et al., 1997; Pelekani and Snoeyink, 1999; Pelekani and Snoeyink, 2000; Ebie et al., 2001; Pelekani and Snoeyink, 2001; Li et al., 2002; Newcombe et al., 2002; Li et al., 2003a; Matsui et al., 2003; Li et al., 2003b; Ding et al., 2008; Redding and Cannon, 2014). 
NIP are small, spherical, particles, and thus have a large area on their outer surface available for adsorption. However, their small size also poses a challenge for full scale treatment with, and subsequent removal of, MIP and NIP particles. Much of the development of full-scale treatment schemes to date has focused on MIP but is equally applicable to NIP. Currently two methods have been investigated for application: incorporation of the particles into macroporous cryogels (Le Noir et al., 2007b; Le Noir et al., 2009; Hajizadeh et al., 2010; Koç et al., 2011; Plieva et al., 2011); and the creation of MIP with a magnetic core (Li et al., 2010; Luo et al., 2011; Xia et al., 2012a; Xia et al., 2012b). MIP cryogels contain pores of varying sizes. Smaller pores allow for contact between MIP, incorporated into the cryogel walls, and the water to be treated. Larger pores allow for a high water flowrate and prevent clogging (Le Noir et al., 2007b; Le Noir et al., 2009; Hajizadeh et al., 2010; Koç et al., 2011; Plieva et al., 2011). MIP with a magnetic core can easily be added to water or wastewater in a batch adsorption process and removed with a strong magnet (Li et al., 2010; Luo et al., 2011; Xia et al., 2012a; Xia et al., 2012b).

This thesis investigated the use of sub-micron polymer particles for water and wastewater treatment. The following questions were addressed:

(1) Can NIP be used to remove EDCs and pharmaceuticals from water and wastewater?

(2) Can NIP be used to remove heavy metals from water and wastewater?

(3) How does (NOM) compete with micropollutants (as modelled by methylene blue dye) for NIP adsorption sites, and how does this compare to activated carbon? 
(4) Are conventional removal methods such as filtration, centrifugation, and ballast flocculation capable of removing NIP particles following treatment?

This thesis is organized into 8 chapters. Chapters 3 and 4 provide background information and materials and methods relevant to the thesis as a whole, and Chapters 5 through 8 are organized in a paper-based format to address each of the above questions in turn. Chapter 9 provides and overall conclusion and directions for future work.

In Chapter 5, NIP were evaluated for removal of EDCs and pharmaceuticals from water and wastewater. Five EDCs: $17 \beta$ estradiol, $17 \alpha$ ethinyl estradiol, estrone, bisphenol A, and diethylstilbestrol were removed from water, both individually, and as a mixture. NIP were able to remove $98 \%$ of spiked E2, $80 \%$ of EE2, $87 \%$ of BPA, and up to $97 \%$ of DES from single-solute solutions. Also, a group of 12 pharmaceuticals with varying chemical characteristics and solubilities: acetaminophen, caffeine, carbamazepine, cloxacillin, diphenhydramine, enrofloxacin, lincomycin, oxacillin, sulfadiazine, sulfamethizole, sulfanilamide, and sulfathiazole were tested for removal from water and wastewater. 9 out of 12 of the pharmaceuticals were removed to some degree from deionized water and 3 out of 7 of the pharmaceuticals measured were removed from wastewater.

In Chapter 6, NIP were assessed for their ability to remove heavy metals from water and wastewater. The NIP were able to achieve high percent removals of lead, copper, zinc, and nickel from river water and wastewater. The NIP showed a strong preference for lead. In river water percent removals were $82 \pm 0.2 \%$ for lead, $46 \pm 0.6 \%$ for copper, $55 \pm 20 \%$ 
for zinc, and $17 \pm 2 \%$ for nickel. Adsorption occurred through an ion-exchange mechanism with the carboxylic acid functional group on the NIP particles. Similarly high removals of $86 \pm 0.1 \%$ of lead, $38 \pm 0.8 \%$ of copper, $28 \pm 1 \%$ of zinc, and $11 \pm 1 \%$ of nickel were observed for a secondary wastewater effluent sample.

In Chapter 7, competition between NOM in natural water and wastewater samples, and micropollutants was evaluated using methylene blue (MB) dye as a size-representative model for micropollutants. MB dye is similar in size to many organic micropollutants, and is able to penetrate into the same pores and occupy the same adsorption sites (Pelekani and Snoeyink, 2000). Thus it was used as a model compound to represent micropollutants. Simultaneous loading with humic acid or wastewater was compared to pre-loading for NIP and activated carbon to determine the competitive mechanisms. The only decrease observed was for simultaneous adsorption with humic acid for NIP (for a $90 \%$ confidence limit). No decreases in adsorption capacity due to competition from NOM were seen for the activated carbon. However, changes in surface chemistry due to adsorbed humic acid and wastewater NOM increased adsorption, and may have cancelled out any decreases due to site competition or pore blocking.

In Chapter 8, removal of the NIP particles following treatment was evaluated. Conventional treatment methods for suspended solids including membrane filtration, centrifugation, and ballasted flocculation were assessed. Centrifugation at 10,000 rpm $(670800 \mathrm{xg})$ for 10 minutes, filtration with a $0.22 \mu \mathrm{m}$ membrane filter, and ballasted flocculation achieved $99 \pm 1 \%, 92 \pm 3 \%$, and $88 \pm 3 \%$ removal, respectively, and were all 
effective for removal of NIP particles. In contrast to the use of cryogels or magnetic particles for application of NIP, these treatment methods are already in use at many treatment plants, and NIP could be incorporated into a treatment scheme with few structural changes. This would be a viable option particularly for the treatment of industrial wastewaters and side streams that have smaller flows and higher EDCs and heavy metal concentrations.

The following list provides details regarding author information and publication of sections of this thesis.

- Chapter 1: Introduction

○ For thesis purposes only.

- Chapter 2: Hypotheses

○ For thesis purposes only.

- Chapter 3: Background

- Section 3.2 was published as a review paper in the journal of Environmental Science and Pollution Research (Murray and Ormeci, 2012). My contribution was to write the review paper.

- Chapter 4: Materials and Methods

○ For thesis purposes only.

- Chapter 5: Use of Non-Imprinted Polymers for Removal of Endocrine Disrupting Compounds and Pharmaceuticals from Water and Wastewater

- Chapter 5 is in preparation for submission to a journal. My contribution was to conduct the experiments, analyze the data, and write the paper. I am the 
first author of the paper, and my supervisor, Dr. Banu Örmeci and Dr. Edward Lai from the Department of Chemistry are the coauthors. I received some training and help using the LC/MS/MS from Ammar Saleem and Da Chen. Some samples were also sent to Axys Analytical Services for analysis, as indicated in the chapter.

- Chapter 6: Use of Non-Imprinted Polymers for Removal of Heavy Metal Ions from Water and Wastewater

○ Chapter 6 has been submitted to Water Research. My contribution was to conduct the experiments, analyze the data, and write the paper. I am the first author of the paper, and my supervisor, Dr. Banu Örmeci is the coauthor. Heavy metal analysis was carried out at the ICP-MS facility at the University of Ottawa.

- Chapter 7: Competitive Effects Of Humic Acid and Wastewater On Adsorption Of Methylene Blue Dye By Activated Carbon And Non-Imprinted Polymers

- Chapter 7 is in preparation for submission to a journal. My contribution was to conduct the experiments, analyze the data, and write the paper. I am the first author of the paper, and my supervisor, Dr. Banu Örmeci is the coauthor. Adsorbent characterization was conducted by Engineering Performance Solutions Labs.

- Chapter 8: Investigation of Physical Separation Processes for Removal of NonImprinted Polymers Following Water and Wastewater Treatment 
- Chapter 8 is in preparation for submission to a journal. My contribution was to conduct the experiments, analyze the data, and write the paper. I am the first author of the paper, and my supervisor, Dr. Banu Örmeci is the coauthor. Microscope imaging was conducted at the Carleton University Nano Imaging Facility.

- Chapter 9: Conclusion

- For thesis purposes only.

This thesis provides a significant contribution to the field in its scope, and the range of contaminants tested with NIP as well as its depth in investigating competitive mechanisms, and methods for removal of NIP and MIP following treatment. Molecularly imprinted polymers (MIP) are conventionally used as a solid-phase extraction media for analytical chemistry (Masqué, 2001). In recent years, researchers have begun to study the use of MIP for water and wastewater treatment (Meng et al., 2005; Le Noir et al., 2007a; Randhawa et al., 2007; Le Noir et al., 2007b; Lin et al., 2008; Yu et al., 2008; Zhongbo and Hu, 2008; Deng et al., 2009; Fernández-Alvarez et al., 2009; Li et al., 2009; Hajizadeh et al., 2010; Krupadam et al., 2010; Li and Lai, 2010; Guo et al., 2011; Luo et al., 2011; Xie et al., 2011; Dai et al., 2012; Venkatesh et al., 2014). However, our group was among the first to study the use of MIP for water and wastewater treatment, and is the first to investigate the use of non-imprinted polymers (NIP) for water and wastewater treatment. MIP are designed to target a specific contaminant, whereas NIP can be applied to a wide range of water and wastewater contaminants. In Chapter 4, NIP were investigated as a potential treatment for removal of a wide range of endocrine disrupting compounds (EDCs) and pharmaceuticals 
from water and wastewater. Previously, researchers investigated the use of MIP prepared with a single template (Meng et al., 2005; Le Noir et al., 2007a; Randhawa et al., 2007; Le Noir et al., 2007b; Lin et al., 2008; Yu et al., 2008; Zhongbo and Hu, 2008; Deng et al., 2009; Fernández-Alvarez et al., 2009; Li et al., 2009; Hajizadeh et al., 2010; Krupadam et al., 2010; Li and Lai, 2010; Guo et al., 2011; Luo et al., 2011; Xie et al., 2011), or several templates simultaneously (Dai et al., 2012; Venkatesh et al., 2014). This technique requires the identification and intentional targeting of individual micropollutants. NIP were evaluated for their potential to remove a wide variety of micropollutants without the need to prepare different polymers for each. In Chapter 6, the same NIP used in Chapter 5 to treat EDCs and pharmaceuticals were further evaluated for their ability to treat heavy metals. Ion imprinted polymers have previously been investigated for treatment of heavy metals, but, like MIP, IIP must be prepared to target each metal ion individually (Branger et al., 2013). NIP were investigated for their ability to treat a range of heavy metals without the imprinting process. In Chapter 7, competitive effects of humic acid and wastewater were investigated using methylene blue dye as a model compound representing a micropollutant. NIP and powdered activated carbon were tested and compared. Because NIP, like activated carbon, are non-specific adsorbents, and do not contain the template specific cavity that MIP do, competition with humic acid or natural organic matter present in wastewater pose a potential challenge for application of NIP. Previous researchers have compared the efficiency of MIP in deionized water with that in natural waters or wastewaters (Le Noir et al., 2007b; Lin et al., 2008; Krupadam et al., 2010; Xie et al., 2011), but have not investigated mechanisms of competition and have not studied that competition in depth. Also, none of these researchers focused specifically on NIP. In 
Chapter 8, removal of MIP and NIP with physical separation processes following treatment was investigated. Previous researchers have investigated the incorporation of MIP into cryogels for application (Le Noir et al., 2007b), or the preparation of MIP with magnetic cores which can be separated from solution with the application of a magnetic field (Li et al., 2010; Luo et al., 2011; Xia et al., 2012a; Xia et al., 2012b), but removal with conventional methods already in use in the water and wastewater industries have not been investigated.

\subsection{REFERENCES}

Alexander, C. 2006 Molecular Imprinting Science and Technology: a Survey of the Literature for the Years up to and Including 2003. Journal of Molecular Recognition, 19 (2), 106-80.

Ashby, J., E. Houthoff, S. J. Kennedy, J. Stevens, R. Bars, F. W. Jekat, P. Campbell, et al. 2011 Commentary The Challenge Posed by Endocrine-Disrupting Chemicals Health. Environmental Health,.

Branger, C., W. Meouche, and A. Margaillan. 2013 Recent Advances on Ion-Imprinted Polymers. Reactive and Functional Polymers, 73 (6), 859-875.

Dai, C.-M., J. Zhang, Y.-L. Zhang, X.-F. Zhou, Y.-P. Duan, and S.-G. Liu. 2012 Selective Removal of Acidic Pharmaceuticals from Contaminated Lake Water Using Multi-Templates Molecularly Imprinted Polymer. Chemical Engineering Journal, 211-212 302-309. 
Deng, S., D. Shuai, Q. Yu, J. Huang, and G. Yu. 2009 Selective Sorption of Perfluorooctane Sulfonate on Molecularly Imprinted Polymer Adsorbents. Frontiers of Environmental Science \& Engineering in China, 3 (2), 171-177.

Ding, L., V. L. Snoeyink, B. J. Mariñas, Z. Yue, and J. Economy. 2008 Effects of Powdered Activated Carbon Pore Size Distribution on the Competitive Adsorption of Aqueous Atrazine and Natural Organic Matter. Environmental Science \& Technology, 42 (4), 1227-31.

Ebie, K., F. Li, Y. Azuma, A. Yuasa, and T. Hagishita. 2001 Pore Distribution Effect of Activated Carbon in Adsorbing Organic Micropollutants from Natural Water. Water Research, 35 (1), 167-79.

Fernández-Alvarez, P., M. Le Noir, and B. Guieysse. 2009 Removal and Destruction of Endocrine Disrupting Contaminants by Adsorption with Molecularly Imprinted Polymers Followed by Simultaneous Extraction and Phototreatment. Journal of Hazardous Materials, 163 (2-3), 1107-12.

Gillesby, B. E., and T. R. Zacharewski. 1998 Exoestrogens: Mechanisms of Action and Strategies for Identification and Assessment. Environmental Toxicology and Chemistry, 17 (1), 3-14.

Guo, W., W. Hu, J. Pan, H. Zhou, W. Guan, X. Wang, J. Dai, et al. 2011 Selective Adsorption and Separation of BPA from Aqueous Solution Using Novel Molecularly Imprinted Polymers Based on Kaolinite/Fe3O4 Composites. Chemical Engineering Journal, 171 (2), 603-611. 
Hajizadeh, S., H. Kirsebom, I. Y. Galaev, and B. Mattiasson. 2010 Evaluation of Selective Composite Cryogel for Bromate Removal from Drinking Water. Journal of Separation Science, 33 (12), 1752-9.

Koç, İ., G. Baydemir, E. Bayram, H. Yavuz, and A. Denizli. 2011 Selective Removal of 17ß-Estradiol with Molecularly Imprinted Particle-Embedded Cryogel Systems. Journal of Hazardous Materials, 192 (3), 1819-26.

Krupadam, R. J., M. S. Khan, and S. R. Wate. 2010 Removal of Probable Human Carcinogenic Polycyclic Aromatic Hydrocarbons from Contaminated Water Using Molecularly Imprinted Polymer. Water Research, 44 (3), 681-8.

Kumar, A., and I. Xagoraraki. 2010 Pharmaceuticals, Personal Care Products and Endocrine-Disrupting Chemicals in U.S. Surface and Finished Drinking Waters: A Proposed Ranking System. Science of the Total Environment, 408 (23), 59725989.

Le Noir, M., A.-S. Lepeuple, B. Guieysse, and B. Mattiasson. 2007a Selective Removal of 17Beta-Estradiol at Trace Concentration Using a Molecularly Imprinted Polymer. Water Research, 41 (12), 2825-31.

Le Noir, M., F. Plieva, T. Hey, B. Guieysse, and B. Mattiasson. 2007b Macroporous Molecularly Imprinted Polymer/Cryogel Composite Systems for the Removal of Endocrine Disrupting Trace Contaminants. Journal of Chromatography. A, 1154 (1-2), 158-64.

Le Noir, M., F. M. Plieva, and B. Mattiasson. 2009 Removal of Endocrine-Disrupting Compounds from Water using Macroporous Molecularly Imprinted Cryogels in a Moving-Bed Reactor. Journal of Separation Science, 32 (9), 1471-9. 
Li, L., P. a Quinlivan, and D. R. U. Knappe. 2002 Effects of Activated Carbon Surface Chemistry and Pore Structure on the Adsorption of Organic Contaminants from Aqueous Solution. Carbon, 40 (12), 2085-2100.

Li, Q., V. L. Snoeyink, B. J. Mariãas, and C. Campos. 2003a Elucidating Competitive Adsorption Mechanisms of Atrazine and NOM Using Model Compounds. Water Research, 37 (4), 773-784.

Li, Q., V. L. Snoeyink, B. J. Mariñas, and C. Campos. 2003b Pore Blockage Effect of NOM on Atrazine Adsorption Kinetics of PAC: The Roles of PAC Pore Size Distribution and NOM Molecular weight. Water Research, 37 (20), 4863-4872.

Li, Y., and E. P. C. Lai. 2010 Extraction of 17ß-Estradiol in Water Using Non-Imprinted Polymer Submicron Particles in Membrane Filters. Journal of Environmental Sciences, 22 (11), 1820-1825.

Li, Y., X. Li, J. Chu, C. Dong, J. Qi, and Y. Yuan. 2010 Synthesis of Core-Shell Magnetic Molecular Imprinted Polymer by the Surface RAFT Polymerization for the Fast and Selective Removal of Endocrine Disrupting Chemicals From Aqueous Solutions. Environmental Pollution (Barking, Essex : 1987), 158 (6), $2317-23$.

Li, Y., X. Li, Y. Li, J. Qi, J. Bian, and Y. Yuan. 2009 Selective Removal of 2,4Dichlorophenol from Contaminated Water Using Non-Covalent Imprinted Microspheres. Environmental Pollution (Barking, Essex : 1987), 157 (6), 187985. 
Lin, Y., Y. Shi, M. Jiang, Y. Jin, Y. Peng, B. Lu, and K. Dai. 2008 Removal of Phenolic Estrogen Pollutants from Different Sources of Water Using Molecularly Imprinted Polymeric Microspheres. Environmental Pollution, 153 (2), 483-91.

Luo, X., Y. Zhan, Y. Huang, L. Yang, X. Tu, and S. Luo. 2011 Removal of WaterSoluble Acid Dyes from Water Environment Using a Novel Magnetic Molecularly Imprinted Polymer. Journal of Hazardous Materials, 187 (1-3), 27482.

Masqué, N. 2001 Molecularly Imprinted Polymers: New Tailor-Made Materials for Selective Solid-Phase Extraction. TrAC Trends in Analytical Chemistry, 20 (9), $477-486$.

Matsui, Y., Y. Fukuda, T. Inoue, and T. Matsushita. 2003 Effect of Natural Organic Matter on Powdered Activated Carbon Adsorption of Trace Contaminants: Characteristics and Mechanism of Competitive Adsorption. Water Research, 37 (18), 4413-4424.

Meng, Z., W. Chen, and A. Mulchandani. 2005 Removal of Estrogenic Pollutants from Contaminated Water Using Molecularly Imprinted Polymers. Environmental Science \& Technology, 39 (22), 8958-62.

Murray, A. 2009 Evaluation of Molecularly Imprinted and Non- Imprinted Nanoparticles for Removal of Endocrine Disrupting Compounds from Surface Water and Wastewater. Carleton University.

Murray, A., and B. Ormeci. 2012 Application of Molecularly Imprinted and NonImprinted Polymers for Removal of Emerging Contaminants in Water and 
Wastewater Treatment: a Review. Environmental Science and Pollution Research, 19 (9), 3820-30.

Murray, A., B. Ormeci, and E. P. C. Lai. 2011 Removal of 17 Beta-Estradiol (E2) and its Chlorination By-Products from Water and Wastewater Using Non-Imprinted Polymer (NIP) Particles. Water Science and Technology, 64 (6), 1291-1297.

Newcombe, G., M. Drikas, and R. Hayer. 1997 Influence of Characterised Natural Organic Material on Activated Carbon Adsorption: II. Effect on Pore Volume Distribution and Adsorption of 2-Methylisoborneol. Water Research, 31 (5), $1065-1073$.

Newcombe, G., J. Morrison, C. Hepplewhite, and D. R. U. Knappe. 2002 Simultaneous Adsorption of MIB and NOM onto Activated Carbon II: Competitive Effects. Carbon, 40 2147-2156.

Pelekani, C., and V. Snoeyink. 1999 Competitive Adsorption in Natural Water: Role of Activated Carbon Pore Size. Water Research, 33 (5), 1209-1219.

Pelekani, C., and V. L. Snoeyink. 2000 Competitive Adsorption Between Atrazine and Methylene Blue on Activated Carbon: The Importance of Pore Size Distribution. Carbon, 38 (10), 1423-1436.

Pelekani, C., and V. L. Snoeyink. 2001 Kinetic and Equilibrium Study of Competitive Adsorption Between Atrazine and Congo Red Dye on Activated Carbon: The Importance of Pore Size Distribution. Carbon, 39 (1), 25-37.

Pichon, V., and F. Chapuis-Hugon. 2008 Role of Molecularly Imprinted Polymers for Selective Determination of Environmental Pollutants--a Review. Analytica Chimica Acta, 622 (1-2), 48-61. 
Plieva, F. M., H. Kirsebom, and B. Mattiasson. 2011 Preparation of Macroporous Cryostructurated Gel Monoliths, Their Characterization and Main Applications. Journal of Separation Science, 2164-2172.

Randhawa, M., I. Gartner, and C. Becker. 2007 Imprinted Polymers for Water Purification. Journal of Applied Polymer Science, 106 (5), 3321-3326.

Redding, A. M., and F. S. Cannon. 2014 The Role of Mesopores in MTBE Removal with Granular Activated Carbon. Water Research, 56 214-24.

Singh, R., N. Gautam, and R. Gupta. 2011 Heavy Metals and Living Systems: An Overview. Indian Journal of Pharmacology, 43 (3), 246-253.

Venkatesh, A., N. Chopra, and R. J. Krupadam. 2014 Removal of Acutely Hazardous Pharmaceuticals from Water Using Multi-Template Imprinted Polymer Adsorbent. Environmental Science and Pollution Research International, 21 (10), $6603-11$.

World Health Organization. 2012 Pharmaceuticals in Drinking-water. World Health Organization Library,

Xia, X., E. P. C. Lai, and B. Ormeci. 2012a Duo-Molecularly Imprinted Polymer-Coated Magnetic Particles for Class-Selective Removal of Endocrine-Disrupting Compounds from Aqueous Environment. Environmental Science and Pollution Research International,.

Xia, X., E. P. C. Lai, and B. Ormeci. 2012b Ultrasonication Assisted Synthesis of Molecularly Imprinted Polymer Encapsulated Magnetic Nanoparticles for Rapid and Selective Removal of $17 \beta$ Estradiol from Aqueous Environment. Polymer Engineering \& Science, 52 (8), 1775-1783. 
Xie, Y., H. Li, L. Wang, Q. Liu, Y. Shi, H. Zheng, M. Zhang, Y. Wu, B. Lu. 2011 Molecularly Imprinted Polymer Microspheres Enhanced Biodegradation of Bisphenol A by Acclimated Activated Sludge. Water Research, 45 (3), 1189-98.

Yang, M., M. S. Park, and H. S. Lee. 2006 Endocrine Disrupting Chemicals: Human Exposure and Health Risks. Journal of Environmental Science and Health. Part C, Environmental Carcinogenesis \& Ecotoxicology Reviews, 24 (2), 183-224.

Yu, Q., S. Deng, and G. Yu. 2008 Selective Removal of Perfluorooctane Sulfonate from Aqueous Solution Using Chitosan-Based Molecularly Imprinted Polymer Adsorbents. Water Research, 42 (12), 3089-97.

Zhongbo, Z., and J. Hu. 2008 Selective Removal of Estrogenic Compounds by Molecular Imprinted Polymer (MIP). Water Research, 42 (15), 4101-8. 


\section{CHAPTER 2: HYPOTHESES}

A list of the hypotheses for the thesis are provided below.

Chapter 5: NIP were evaluated for their ability to remove a range of endocrine disrupting compounds (EDCs) and pharmaceuticals from water and wastewater.

- NIP will remove both EDCs and pharmaceuticals to varying degrees depending on the solubilities of the compounds.

- Increasing the NIP concentration will increase the removal of both EDCs and pharmaceuticals.

- If NIP are applied to treat the same compounds in wastewater, removal is expected to decrease in comparison to deionized water.

Chapter 6: NIP were evaluated for their ability to remove heavy metals from water and wastewater.

- NIP will remove heavy metals due to ion exchange with the carboxylic acid functional groups on the NIP.

- At higher $\mathrm{pH}$ values, removal of heavy metals with NIP will increase due to an increase in negatively charged ions.

- Removal of heavy metals from river water and wastewater will be lower than that in deionized water due to the presence of competing ions. 
Chapter 7: Methylene blue dye (MB) was used as a model for micropollutants during simultaneous loading and pre-loading experiments with humic acid and wastewater to investigate competitive effects on both NIP and powdered activated carbon.

- NIP and activated carbon will have similar adsorption capacities in deionized water.

- The adsorption capacities of both NIP and activated carbon will decrease in comparison to deionized water in the presence of humic acid or wastewater. The adsorption capacity of activated carbon will decrease more than that of the NIP due to the presence of a large, interconnected series of pores in the activated carbon.

- NIP and activated carbon which are pre-loaded with humic acid or wastewater and then applied for adsorption of MB, will exhibit a further decrease in adsorption capacity beyond that observed for simultaneous loading, due to pore blocking. The difference in adsorption capacity between simultaneous loading and pre-loading will be less for NIP than for activated carbon.

Chapter 8: Centrifugation, filtration, and ballast sand flocculation were tested for removal of NIP from solution. NIP particles were also tested under a variety of acidic and basic conditions.

- Centrifugation, filtration, and ballast sand flocculation will all effectively remove NIP particles from water.

- Increasing centrifuge time or speed will increase removal.

- Decreasing the filtration pore size will increase removal. 
- Use of ballast flocculation as a pre-treatment for centrifugation or filtration will increase removal. 


\section{CHAPTER 3: BACKGROUND}

\subsection{MOLECULARLY IMPRINTED AND NON-IMPRINTED POLYMER PARTICLES}

\subsubsection{Introduction}

Pre-concentration of environmental estrogen samples for analytical evaluation is a necessary step for quantifying concentrations in water and wastewater treatment as well as in natural systems. Solid phase extraction is a common pre-concentration technique used for many applications of trace analysis, and molecularly imprinted polymers (MIP) were first developed as a medium for solid phase extraction for trace analysis purposes (Masqué, 2001).

During most solid phase-extraction procedures, the wide range of contaminants contained in water samples for environmental analysis are co-extracted and complex extraction procedures may be required prior to analysis to isolate specific contaminants. MIP technologies offer a simultaneous pre-concentration and extraction method because the binding sites on the MIPs are designed to remove a specific contaminant from the water system (Masqué, 2001).

MIP technologies are founded on imitation of biological antibody systems. Antibodies function based on specific binding sites where the antigen binds strongly and noncovalently to the antibody (Bauman, 2004). MIP are synthetic antibodies created from cross-linked polymers containing a cavity specific to an analyte. These cavities are created by copolymerization of cross-linking monomers and functional monomers along with an 
imprinting molecule or template. Following polymerization, the template is removed, leaving a cavity specific to the analyte. The MIP's then selectively re-bind to this analyte compound (Sellergren, 1999; Masqué, 2001; Alexander, 2006).

Figure 3-1, shows how MIP's are formed and used. The monomer forms around the template in either covalent or non-covalent binding, and is polymerized with the crosslinker. The template can then be removed, leaving a template-specific cavity, which can be used for removal of template molecules from pure or contaminated samples.
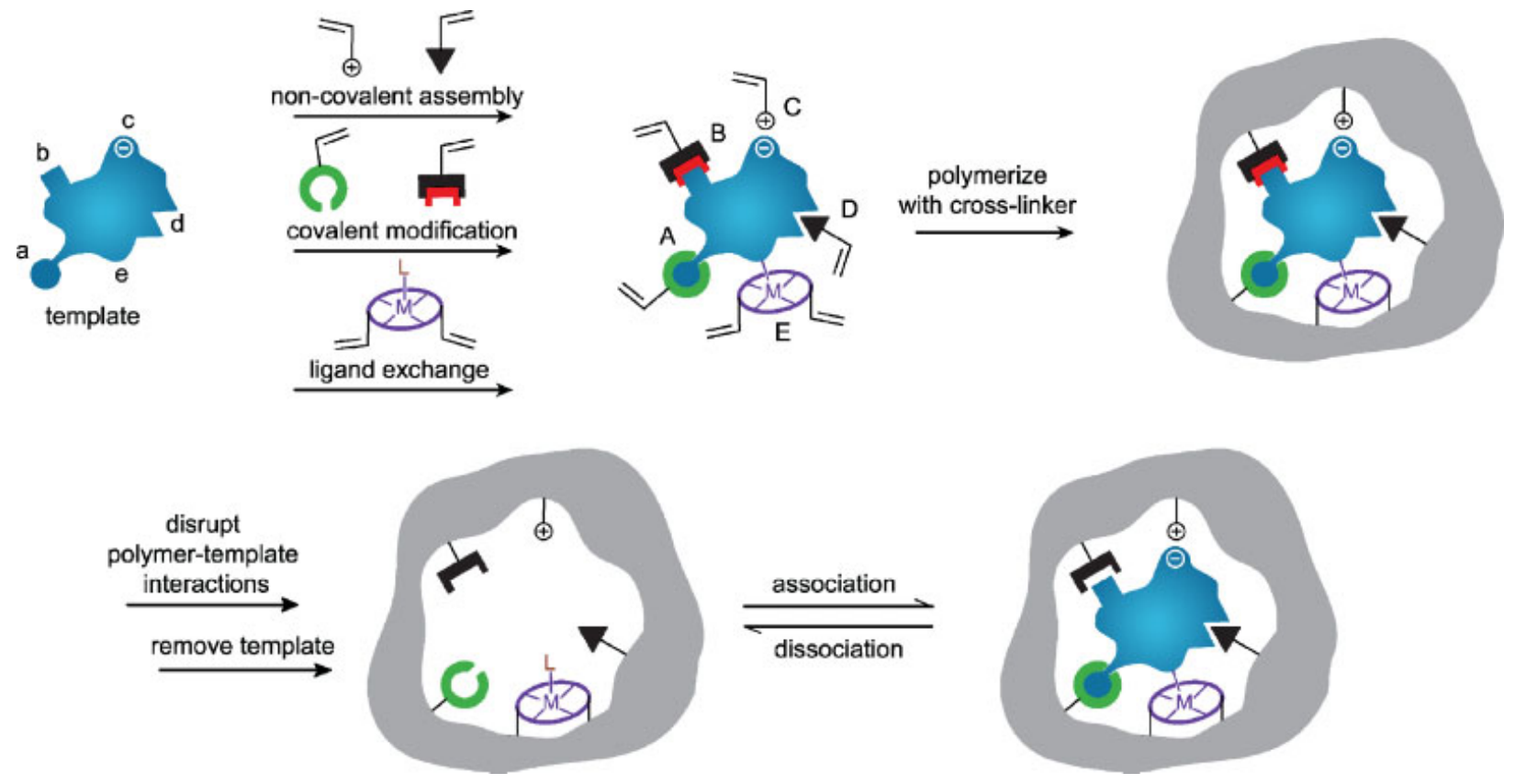

Figure 3-1: Schematic Showing the Formation, Design, and Use of MIP Particles (Alexander, et al., 2006) 


\subsubsection{Design of molecularly imprinted and non-imprinted particles}

There are several approaches for the design of MIP. The optimum approach depends on the template to be used and the intended purpose of the MIP. These approaches include: the covalent approach, the non-covalent approach, the stochiometric non-covalent approach, and the semi-covalent approach. The non-covalent approach was used to create the MIP particles used for this study. Each of these approaches are discussed in detail below.

\subsubsection{The covalent approach for design of molecularly imprinted and non-imprinted particles}

The covalent approach uses templates which are covalently bound to one or more polymer groups. Following polymerization, the template is removed and the binding site is left with the capability of re-forming covalent bonds with template molecules. An advantage of the covalent approach is that functional groups are only associated with the template sites, meaning that binding is highly specific. However, the approach can only be used for a limited number of template molecules such as alcohols, aldehydes, ketones, amines, and carboxylic acids (Alexander, 2006).

\subsubsection{The non-covalent approach for design of molecularly imprinted and non- imprinted polymers}

The non-covalent approach functions by non-covalent forces such as hydrogen-bonding, ion-pairing, and dipole-dipole interactions. The interactions between the functional monomer and template during polymerization are the same as those between the polymer 
and the template during rebinding. This is the most widely used method for MIP production (Alexander, 2006). Methacrylic acid (MAA) is the most commonly used functional monomer for the non-covalent approach and ethylene glycol dimethacrylate (EGDMA) is the most commonly used crosslinking monomer (Sellergren, 1999; Masqué, 2001). Low molecular weight templates with functional groups capable of hydrogen bonding or acidbase reactions with the monomer can be used as templates for the non-covalent approach.

\subsubsection{The stochiometric non-covalent approach for design of molecularly imprinted and non-imprinted polymers}

The stochiometric non-covalent approach is a modification of the non-covalent approach for which the association constant for the reaction between the template and monomer is high and the equilibrium favours polymerization and inclusion of the template into the polymer structure (Alexander, 2006).

\subsubsection{The semi-covalent approach for design of molecularly imprinted and non- imprinted polymers}

The semi-covalent approach uses a combination of the covalent and non-covalent approaches. Initial polymerization takes place with covalent binding between the template

and monomer. Following removal of the template from the polymer structure, rebinding takes place non-covalently. An advantage is higher specificity than the non-covalent approach and a more uniform distribution of binding sites. The template and monomer may be connected directly or using an additional spacer group to avoid crowding of the template site (Alexander, 2006). 


\subsubsection{Design considerations for design of molecularly imprinted and non-imprinted polymers}

The ratio of monomer to template used for construction of MIP is also very important for selectivity. Increasing the amount of monomer increases the amount of analyte bound, but decreases the selectivity if the increase is beyond an optimum ratio. This occurs because excess monomer can accumulate on the surface and provide additional non-specific adsorption sites (Liao et al., 2007).

The yield of imprinted binding sites, and hence the binding capacity of the MIP, is directly related to the strength of the interaction between the functional monomers and the template both before and during polymerization. The thermodynamics of the system, the number and type of monomer interaction sites, the shape of the template, and the rigidity of the cavity are all important factors in determining the strength of interaction (Wulff, 1995; Sellergren, 1999).

The number of imprinted binding sites is affected by the temperature of polymerization. Low temperature polymerization is generally prefered to form the maximum number of imprinted binding sites. Polymerization at a high temperature generally occurrs very quickly producing less reproducible results. Lower temperatures reduce the rate of reaction and increase the quantity of binding sites (Yan and Row, 2006). Side reactions are also minimized at low temperatures (Ensing and De Boer, 1999). 
The number of monomer interaction sites is important because the number of interactions will increase both the binding strength and specificity. Therefore, a functional monomer should be chosen which allows for a maximization of the number of interactions. The strength of these interactions is also important. Care should also be taken to ensure that the monomer-monomer interactions do not compete with template-monomer interactions because, especially if there is no preference for the interactions with the template over the monomer, competition can substantially decrease the number of cavities formed (Sellergren, 1999).

Although MIP polymers can be made with a variety of monomers and cross-linkers, methacrylic acid (MAA) as the monomer and ethylene glycol dimethacrylate (EGDMA) as the cross-linker are widely recognized as the least expensive and most efficient choices (Wulff, 1995; Ensing and De Boer, 1999). However, other combinations have been used. For example, Bravo et al (2009) studied MIP with MAA as a functional monomer but with either EGDMA or divinylbenzene (DVB) as cross-linkers for MIP with EE2 as the template. They found that the MIP formed with a mixture of the two cross-linkers or with DVB alone were more specific than those using EGDMA as the cross-linker (Bravo et al., 2007).

Andersson et al (1996) studied the formation of MIP using pyridine, which is only capable of forming one hydrogen bond site with carboxylic acid groups in the monomer. It was expected that there would be no evidence of specific binding for this polymer, because previous research had indicated that two hydrogen bonds or at least other hydrophobic or 
Van der Waals interactions must occur. However, there was limited specificity but increased adsorption for pyridine in comparison with similar compounds. The conclusion was that there were unknown interactions occurring, but the strength of the specificity was dependent on the number and type of interactions (Andersson et al., 1996).

Another consideration is template shape. Templates other than the analyte of interest may be used (for reasons discussed in Section 3.1.5.1 below); however, the shape must be close to that of the analyte for interaction between the monomer and analyte to occur. Rebinding will occur with a much lower entropy loss if the template and the analyte are similar in shape. This is because entropy is lost due to shape changes in the site and template upon bonding (Sellergren, 1999).

Structural integrity or rigidity of the template sites is also important. Functional groups must be positioned in a way that allows for access by the template. This is achieved by cross-linking, and cross-linking must be above $50 \%$ for recognition between the cavity and the template to occur (Sellergren, 1999). Cross-linking between 70-90\% is ideal and will help the polymer cavities retain their shapes (Masqué, 2001). The porosity of the polymercavity system must also be maintained to ensure that binding is not diffusion-limited (Sellergren, 1999). 


\subsubsection{Types of binding and adsorption that may occur on molecularly imprinted and non-imprinted polymers}

Zhang and $\mathrm{Hu}$ (2008) proposed a model containing three separate types of binding to explain interactions between estrogenic contaminants (E1, E2, EE2, and BPA) and MIP. The binding types included: (1) specific adsorption; (2) semi-specific adsorption; and (3) non- specific adsorption (Zhongbo and Hu, 2008). Figure 3-2, illustrates the types of interactions that can occur for each of the aforementioned types of binding sites.

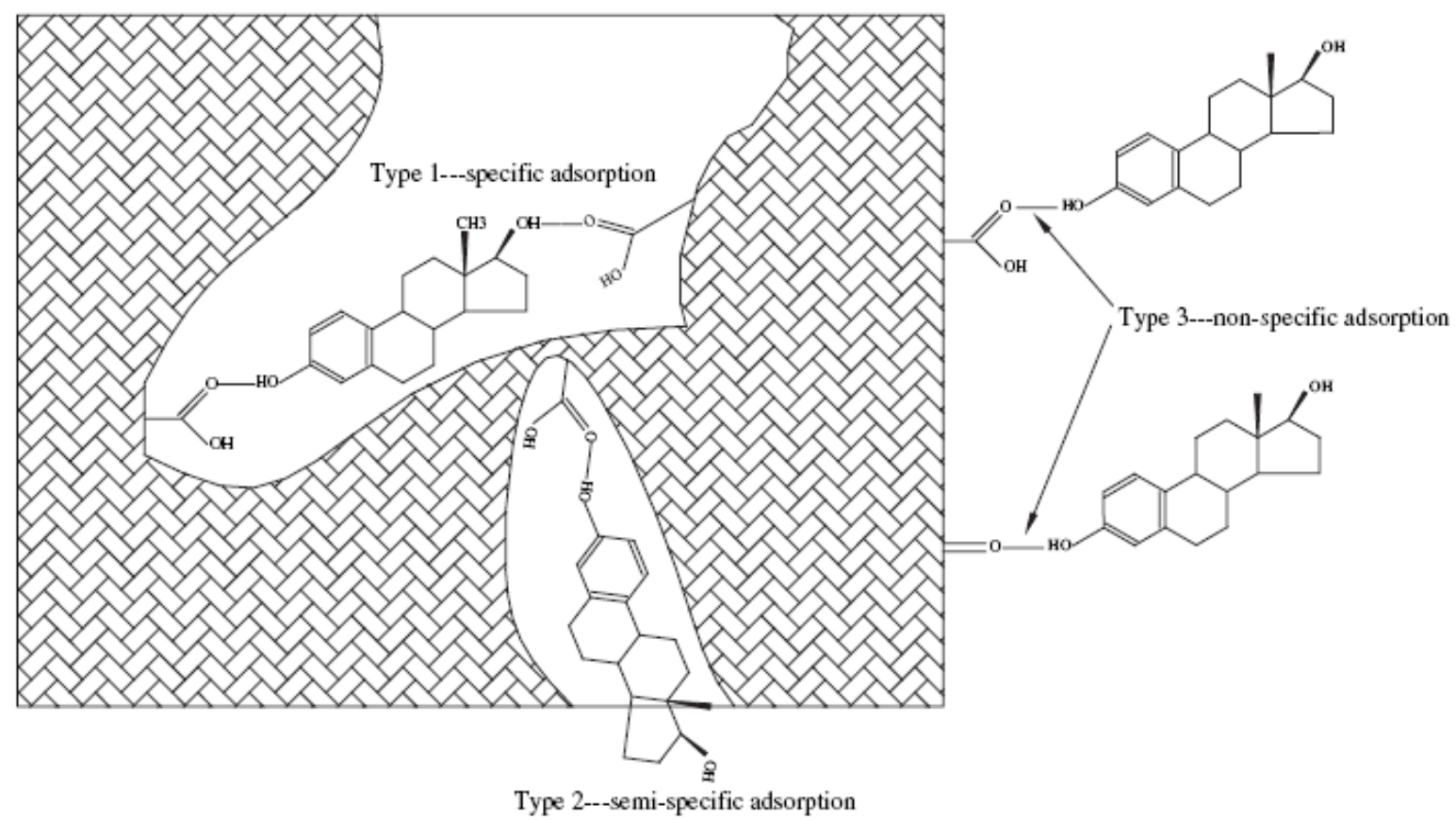

Figure 3-2: Specific, semi-specific and non-specific adsorption of endocrine disruptors to MIP prepared with an E2 template (Zhang \& Hu, 2008)

Specific adsorption, as shown in the figure, can only occur for binding of the template molecule. For site-specific binding to be affective, the shape of the binding site must closely match that of the template. There are also several thermodynamic considerations 
which determine whether or not binding will take place as well as how specific the binding will be. The type and strength of the bond formed determines the selectivity of the cavity. For example, if the template binds to the receptor using hydrogen bonding or electrostatic interactions, these are much stronger than Van der Waals or hydrophobic interactions and there will be a preference for that template over other competing compounds (Konig, 1995).

Thermodynamic considerations are important to determine whether or not any binding of the template will take place. Therefore there are three factors which must be considered in determining whether or not specific binding will occur: (1) the template and cavity must have complimentary molecular shapes; (2) there must be available non-covalent binding sites in the template (this will lead to a favourable entropy of reaction); and (3) molecular rotation following binding should be minimized (this will lead to a favourable enthalpy) (Konig, 1995).

Semi-specific adsorption sites can host either specific or non-specific adsorption due to the shape of their binding cavities. For MIP with an E2 template, both estrogenic and other compounds could bind to these sites; however, those compounds with a benzene ring would have a competitive advantage for the occupation of these sites because of their similarity to E2. Zhang and $\mathrm{Hu}$ (2008) attributed semi-specific binding to the use of excess functional monomers during polymer synthesis. Functional monomers were added at a ratio of 4.8:1 compared to the E2 template. This was done to encourage the creation of more specific binding sites. The stochiometric requirement for the functional monomer to template ratio 
is $2: 1$ for the polymer formulation used. Excess functional monomers could remain at the surface of the polymer, thus providing sites for either semi-specific or non-specific adsorption (Zhongbo and $\mathrm{Hu}, 2008)$.

Non-specific adsorption refers to surface adsorption and can occur due to hydrophobic interactions and Van der Waals forces in aqueous solutions since most endocrine disruptors are hydrophobic. However, non-specific interactions were also attributed to the presence of the cross-linker which contained carbonyl groups which may have created conditions for hydrogen bond formation on the surface of the polymer where any compound capable of forming hydrogen bonds with a carboxyl or carbonyl group may have adsorbed (Zhongbo and $\mathrm{Hu}, 2008$ ).

\subsubsection{Thermodynamics of specific and non-specific binding for molecularly imprinted and non-imprinted polymers}

Thermodynamically, there are two aspects of binding which must be considered: enthalpy and entropy. Enthalpy results from energy changes which occur with interactions between the adsorbent and substrate. For imprinted polymers, this energy is very high because it is the result of the imprinted memory of the polymer. The adsorption entropy is the result of a decreased disorder following the interaction between the substrate and MIP, therefore, the enthalpy is the guiding thermodynamic factor and the energy of the binding site must be high enough for spontaneous binding to occur. Increased temperature can further increase the energy available and results in a higher level of binding because lower energy binding sites will become available to the substrate (Fang and Li, 2007). 
Fang and Li (2007), studied NIP and MIP imprinted with bisphenol-A. They found that there was a higher desorption energy for BPA from MIP versus NIP, indicating specific binding sites with a higher energy than non-specific adsorption sites. They took SEM (scanning electron microscope) images indicating a smooth surface for NIP and a pitted surface for MIP, indicating clearly visible specific binding sites. Furthermore, infrared spectrum from each of the MIP with the template removed and NIP showed that MIP with the template removed were similar to NIP, indicating complete removal of the template. Also, for binding on NIP, BPA and structurally similar compounds exhibited similar binding efficiencies but BPA exhibited higher binding efficiencies for MIP, indicating specific binding sites (Fang and Li, 2007).

Li et al (2009) also studied the interactions and thermodynamic differences for specific and non-specific binding. They found two different energy levels indicated by two kinetic adsorption steps for adsorption of 2,4-dichlorophenol to MIP. The adsorption exhibited a quick adsorption step followed by a relatively slower step. The quick step was attributed to ionic interactions and the slow to less-specific hydrogen bonding. They gave two reasons for specificity. The first was that 2,4-dichlorophenol was larger in size than the other compounds tested and thus could fit more specifically into the holes left by the removal of the template and the second was that the interaction energies were highest for 2,4dichlorophenol (Li et al., 2009).

Adsorption also depends on the cohesive energy holding the endocrine disruptors in the mobile phase. Successful adsorption requires a force field surrounding the surface of the 
adsorbent which will reduces the potential energy of the adsorbate below that experienced in the fluid phase (Karge and Weitkamp, 2008). The cohesive forces typically experienced by molecules in the liquid phases are Van der Waals or hydrogen bonding forces (Chiou, 2002). The forces typically exerted by the surface of imprinted or non-imprinted polymers are similar in nature, but the specific binding sites on MIP typically form at least two hydrogen bonds with the template molecules, which are stronger in nature, and provide a greater attraction than that of the non-specific sites.

\subsubsection{Molecularly imprinted particles for solid phase pre-concentration for analytical analysis}

The precursor to molecularly imprinted polymers (MIP) was first created in the 1930's using silica matrices. Silica particles were prepared in the presence of benzene, toluene, or xylene. The additive was removed, and subsequent adsorption was shown to be greater than for non-imprinted particles. During the 1930's, little was understood about how antibodies worked, and the mechanisms behind these synthetic antibodies were not well understood. However, since then, a great deal of work has been done in the field and the research has reached a point where it has many applications including analytical solid phase pre-concentration (Alexander, 2006).

Solid phase extraction (SPE) is the most advanced application of molecularly imprinted polymers (Alexander, 2006). SPE involves selective adsorption of analyte compounds into the cavities of MIP particles followed by desorption with a desorption agent and subsequent analytical analysis. The use of MIP for SPE has the advantage that it can simultaneously 
be used for sample cleanup and pre-concentration. The sample is cleaned because the analyte is selectively adsorbed from the solution onto the MIP. Furthermore, a lower volume of the desorption agent can be used than the initial sample volume, and preconcentration is achieved (Alexander, 2006).

\subsubsection{Bleeding of the template molecule during analytical pre-concentration and analysis}

Complete removal of the template from MIP can be very difficult and involves numerous washing steps. In fact, often even following extensive washing steps, leaching of template from the polymer will still occur (Pichon, 1997). Generally more than $1 \%$ of the template remains bound following extensive washing. Bleeding of the template following solidphase pre-concentration and subsequent desorption of the analyte will lead to falsely high results (Sellergren, 1999).

One solution to this phenomenon is the use of a structural analogue similar to the analyte for use as a template during the preparation of the polymer. A template which does not interfere with the analyte during analysis should be chosen and it would not matter if contamination or leaching of the template occurred, the analyte could still be effectively measured (Masqué, 2001). However, this is not a solution for water treatment applications where the purpose is removal of trace organic contaminants from the water. Replacement of one contaminant with another similar contaminant would not be sufficient for water treatment. Thermal treatment or more effective washing can also be used to reduce bleeding (Sellergren, 1999). 
Peng-Ju et al (2007) developed a procedure of accelerated solvent extraction for more efficient template removal to remove a nicotine template from MIP. They found an increase in extraction efficiency with elevated temperature up to a temperature of $80{ }^{\circ} \mathrm{C}$ and were able to remove $94.2 \%$ of the template in 70 minutes using an optimized mixture of methanol and acetic acid (Peng-Ju et al., 2007).

Zhongbo and $\mathrm{Hu}$ (2008) created a mass balance for the template E2 removed from their MIP particles. The particles were to be used for water treatment purposes. Creation of a mass balance is the only way to confirm that the entirety of the template is removed from the MIP following polymerization, and the importance of this cannot be overstated for water treatment purposes where addition of harmful compounds into the water cannot be tolerated (Zhongbo and Hu, 2008).

\subsection{APPLICATION OF MOLECULARLY IMPRINTED AND NON-IMPRINTED POLYMERS FOR REMOVAL OF EMERGING CONTAMINANTS IN WATER AND WASTEWATER TREATMENT}

\subsubsection{Introduction}

Micropollutants are typically released into the environment via wastewater discharges and land application of biosolids, and contaminate the groundwater and surface waters that are used as drinking water resources. The concentrations of these contaminants are extremely low, typically at levels of parts per billion (ppb) or parts per trillion (ppt), which makes their treatment difficult. However, several laboratory and field studies have shown that even at very low levels, these compounds can accumulate in aquatic organisms and adversely affect growth and reproduction. 
Endocrine disrupting compounds (EDCs) are one class of micropollutants, composed of chemicals capable of mimicking or blocking the endogenous hormonal system of animals. A recent literature review on EDCs indicated that suspected human health risks include reproductive disorders (e.g., polycystic ovary syndrome, decreased human sperm counts and quality, male reproductive tract abnormalities, changed male birth rates and sex ratio, increased spontaneous abortion rates, and endometriosis), immune and hormonal disorders (e.g., immune alterations, diabetes, and precocious puberty), and neurobehavioral disorders (e.g., prenatal exposure, infant neurodevelopment, neuromuscular delays, hypothyroidism and altered thyroid hormone) (Yang et al., 2006). The long-term effects of emerging contaminants on humans are still largely unknown. It has been suggested that increases in breast, testicular, and prostate cancers reported over the past forty years are related to EDCs in the environment (Gillesby and Zacharewski, 1998; Ashby et al., 1997).

Effects of EDCs on aquatic ecosystems are less controversial and more widespread than the effects on humans. Aquatic organisms are constantly exposed to untreated surface waters contaminated with wastewater effluents, whereas humans only drink about $2 \mathrm{~L}$ of treated drinking water each day. The effects on aquatic ecosystems are numerous but include: an increase in the proportion of females relative to males (Bögi et al., 2003); delays in the first spawning age leading to reduced fertilization success (Segner et al., 2003); reduced rates of sperm and egg production and release; reduced gamete quality; failure to reach sexual maturity; and unnatural sex reversal (Helfman, 2007). 
Both water and wastewater treatment plants are expected to remove micropollutants, but in reality they are neither designed nor equipped for this task. Several studies have recently compared the effectiveness of various treatment and removal processes for micropollutants. Coagulation and flocculation are not very effective at removing micropollutants (Vieno et al., 2006), but some removal through co-precipitation with solid particles and by adsorption onto the surface of particles is possible (Snyder et al., 2007). Activated carbon is effective at removing hydrophobic organic contaminants, and the majority of hydrophobic micropollutants have shown very good removals. However, activated carbon treatment is typically reserved for drinking water applications and not for wastewater; and a number of factors can affect the removal efficiency such as adsorption kinetics, adsorbate concentration, and water quality (Fuerhacker et al., 2001). Oxidation of micropollutants with strong chemical oxidants (e.g., ozone) and advanced oxidation processes (e.g., $\mathrm{UV}-\mathrm{H}_{2} \mathrm{O}_{2}$ ) is also possible. However, these processes do not completely remove contaminants and instead result in the formation of oxidation products which in some cases might be more toxic or estrogenic than the initial compound (e.g., atrazine and diethylatrazine) (Adams and Randtke, 1992). During biological wastewater treatment, micropollutants are partially degraded, and the degradation rate can be improved by increasing the sludge retention time to at least 15 days (Clara et al., 2005). Micropollutants are not completely removed; however, and they are simply broken into smaller compounds with the potential to be more toxic or estrogenic than the parent compound. In terms of membrane processes, reverse osmosis (RO) and nanofiltration (NF) offer unique benefits for contaminant removal as high rejections can be obtained without formation of byproducts. On the down side, their extremely high capital and operational costs make 
them economically unfeasible. Braeken et al. (2009) studied several implementation strategies for nanofiltration treatment of EDCs for a ten year return period and found all to be unfeasible (Braeken and Van der Bruggen, 2009).

In summary, current water and wastewater treatment technologies have limitations for removing emerging contaminants, and there is a need for new technologies that are both effective and economically feasible. Wastewater discharges are the main source of micropollutants, and it is particularly important to remove emerging contaminants from wastewater effluents as this would help to stop the problem at the source. The goal of this section is to provide a critical review of recent research to investigate the potential use of molecularly imprinted (MIP) and non-imprinted polymers (NIP) as a treatment method for removal of micropollutants from water and wastewater.

\subsubsection{What are molecularly imprinted and non-imprinted polymers?}

MIP are prepared from cross-linked polymers containing cavities specific to an analyte. These cavities are created by copolymerization of cross-linking monomers and functional monomers along with an imprinting molecule or template. Following polymerization, the template is removed, leaving a cavity specific to the analyte. The MIP then selectively rebinds to the analyte compound (Masqué, 2001; Alexander, 2006). In a way, MIP technologies imitate biological antibody systems which function on the basis of specific binding sites where an antigen binds strongly to an antibody (Alexander, 2006). 
MIP are conventionally used as a solid-phase extraction media for analytical chemistry. During most solid phase-extraction procedures, the wide range of contaminants contained in water samples for environmental analysis are co-extracted and complex extraction procedures may be required to isolate specific contaminants. MIP technologies offer a simultaneous pre-concentration and extraction method because the binding sites on the MIPs are designed to remove a specific contaminant from the water system (Masqué, 2001).

NIP, on the other hand, are cross-linked polymeric materials that have macropores containing adsorption sites for organic molecules. They are synthesized using the same procedure as MIP, but in the absence of a template. Hence, they have the same chemical properties as MIP but contain no specific cavities (Pichon and Chapuis-Hugon, 2008). NIP exhibit strong non-specific binding which is attributed to hydrophobic interactions between organic compounds and polymers. In MIP studies, NIP particles are studied as a control against MIP particles to compare non-specific binding to template specific binding.

\subsubsection{Potential use of MIP for water and wastewater treatment}

In recent years, several researchers have begun to study the use of MIP for water and wastewater treatment (Meng et al., 2005; Zhongbo and Hu, 2008; Hajizadeh et al., 2010; Deng et al., 2009; Luo et al., 2011; Guo et al., 2011; Li et al., 2009; Yu et al., 2008; Lin et al., 2008; Krupadam et al., 2010; Le Noir et al., 2007a; Le Noir et al., 2007b; FernándezAlvarez et al., 2009; Randhawa et al., 2007; Xie et al., 2011; Murray, 2009; Murray et al., 2011). MIP are advantageous for treatment of trace contaminants because they can be 
specifically designed to remove one or a group of target compounds. This is an advantage over non-specific technologies such as activated carbon, which may be consumed removing large amounts of non-trace contaminants from the water.

MIP researchers have studied the isotherms, kinetics, performance under varying conditions, application, and regeneration of MIP for water and wastewater treatment. A summary of their findings are provided below.

\subsubsection{Adsorption characteristics of MIP}

\subsection{Adsorption isotherms}

Two common models which are used to represent equilibrium batch adsorption tests are the Langmuir and Freundlich isotherms. The Langmuir isotherm is typically used to describe cases where the adsorbate forms a monolayer on the adsorbent. The Langmuir isotherm model assumes that there is no interaction between adjacent adsorption sites and that the energy of adsorption is the same for all adsorption sites. The Freundlich model is typically applied where there are heterogeneous adsorption sites with different binding energies. Interestingly, different researchers have found that their results with MIP were best represented by different models. This may be because different researchers used different procedures to make their MIP and used different templates or adsorbates.

Meng et al. (2005), Zhongbo et al. (2008), Hajizadeh et al. (2010), and Luo et al. (2011) all found good correlation between experimental isotherm data collected for different types of MIP (with different templates) and the Freundlich isotherm model. This confirmed 
theories that MIP contain specific binding sites, semi-specific binding sites, and nonspecific binding sites all with different binding energies.

In contrast, Guo et al. (2011), Li et al. (2009), and Deng et al. (2009) all found that their experimental data were best described by the Langmuir isotherm model, and Yu et al. (2008) found that both models could be used. Li et al. (2009) explained that the Langmuir isotherm model was a better fit for adsorption onto MIP because the template cavities on the MIP surface were homogeneous. It is likely that the template cavities on the MIP surface are more homogeneous than the binding sites on activated carbon which are available to the adsorbates through a heterogeneous network of pores, but the adsorption sites themselves may still be heterogeneous.

\subsection{Adsorption kinetics}

Yu et al. (2008), Hajizadeh et al. (2010), and Guo et al. (2011) all compared pseudo-first order and pseudo-second order kinetic models to describe adsorption for MIP and NIP using different MIP and templates and all found that the pseudo second-order model better described the adsorption kinetics. This was because they all noticed an area of fast adsorption followed by a region of much slower adsorption. Li et al. (2009) also observed this trend, but did not fit a kinetic model to their data.

The time required to reach equilibrium varied for different MIP and between MIP and NIP. Deng et al. (2009) using MIP created with a perfluorooctane sulfonate (PFOS) template found that equilibrium was reached within 5 minutes and attributed this to the small particle 
size of their MIP $(74 \mu \mathrm{m})$ and monolayer adsorption, which fit a Langmuir isotherm. In contrast, Yu et al. (2008) found that their MIP, which were also created with a PFOS template, but which were a chitosan based MIP, took $32 \mathrm{~h}$ to reach equilibrium while NIP took 16h. The explanation given was that MIP contained a large number of micropores and it took the PFOS longer to diffuse into these pores.

\subsubsection{Effect of water characteristics on MIP performance}

\subsection{Effect of $p H$}

Changing the $\mathrm{pH}$ of the solution can lead to ionization of the adsorbate or changes in the surface charge of the MIP. Therefore, the effect of $\mathrm{pH}$ is specific to the MIP used and must be studied separately for each case (Luo et al., 2011). However, several MIP researchers have studied the effects of varying $\mathrm{pH}$ on MIP performance and these studies can provide clues about the performance of other MIP in the future. For example, all of the papers considered in this review noted optimum pH's which were acidic (Yu et al., 2008; Deng et al., 2009; Hajizadeh et al., 2010) or neutral (Guo and Krasner, 2009; Li et al., 2009; Zhang et al., 2011).

Lin et al. (2008) investigated the effects of varying $\mathrm{pH}$ on adsorption by MIP imprinted with bisphenol A (BPA) with a 4-vinyl pyridine (4-VP) monomer and found that the optimal removal occurred at $\mathrm{pH} 5$ and the lowest removal efficiency occurred at a neutral $\mathrm{pH}$. Yu et al. (2008) found that increasing the $\mathrm{pH}$ of the solution decreased the amount adsorbed by a chitosan-based MIP imprinted with PFOS until, at a $\mathrm{pH}$ of 10, almost no 
adsorption occurred. Deng et al. (2009) also found that the amount adsorbed decreased with increasing $\mathrm{pH}$ for MIP imprinted with perfluorooctane sulfonate with the most removal occurring at $\mathrm{pH}$ 3. Interestingly, Yu et al. (2008) found that almost no removal occurred at $\mathrm{pH} 10$ while Deng et al. (2009) found that at a $\mathrm{pH}$ of $9,50 \%$ of the PFOS was still removed. Yu et al. (2008) suggested that the significant effect of $\mathrm{pH}$ for their MIP indicated that the adsorption was predominately due to electrostatic attractions because only electrostatic attractions are sensitive to $\mathrm{pH}$.

Hajizadeh et al. (2010) reported that bromate adsorption by MIP had an optimum at $\mathrm{pH} 3$. They used chitosan MIP and the optimum $\mathrm{pH}$ was attributed to the protonation of chitosan to form $\mathrm{NH}_{3}{ }^{+}$groups, increasing adsorption. Lastly, Luo et al. (2011) studied MIP designed for the removal of acidic dyes. They found that the removal efficiency increased with decreasing $\mathrm{pH}$. They attributed this to bromide ions on the MIP accepting hydrogen ions from the solution giving the MIP a more positive charge which attracted sulfonate ions in the dyes.

For water and wastewater treatment applications, the conditions are generally neutral or near-neutral. Therefore, MIP that are most effective under neutral conditions are of special interest. Li et al. (2009), working with 2,4-dichlorophenol (2,4-D) imprinted MIP, recorded the maximum removal of $83.2 \%$ at $\mathrm{pH} 7$. They noted that since the main binding force was hydrogen bonding, minimizing the concentrations of $\mathrm{H}^{+}$and $\mathrm{OH}^{-}$ions in solution would maximize the removal. Zhang and $\mathrm{Hu}$ (2008) observed no changes in adsorption up to a $\mathrm{pH}$ of 11 , where the removal decreased. The decreased adsorption at $\mathrm{pH} 11$ was attributed 
to either ionization of the target compound or a change in the charge of the carboxyl groups on the methacrylic acid monomer. Guo et al. (2011) also observed their optimum removal at a $\mathrm{pH}$ of 7 working with a magnetic BPA-imprinted MIP.

\subsection{Effect of ionic strength}

Adding ions to the solution can lead either to increased adsorption by decreasing the solubility of the adsorbate in solution or to decreased adsorption by acting like a shield and decreasing the electrostatic attraction between the MIP and the template.

Yu et al. (2008) found that adsorption increased for MIP and NIP with increasing $\mathrm{NaCl}$ concentration starting at $50 \mathrm{mmol} / \mathrm{L}$ for MIP and $20 \mathrm{mmol} / \mathrm{L}$ for NIP. Lin et al. (2008) tested adsorption in the presence of $\mathrm{NaCl}, \mathrm{NaNO}_{2}, \mathrm{Na}_{2} \mathrm{HPO}_{4}$, and $\mathrm{CaCl}_{2}$ at concentrations of 1,10 , and $50 \mathrm{mmol} / \mathrm{L} .1$ or $10 \mathrm{mmol} / \mathrm{L} \mathrm{NaCl}, \mathrm{NaNO}_{2}$, and $\mathrm{CaCl}_{2}$ did not affect the removal efficiency, but 1 or $10 \mathrm{mmol} / \mathrm{L}$ of $\mathrm{NaNO}_{2}$ increased removal efficiency. The increased removal efficiency with 1 or $10 \mathrm{mmoles} / \mathrm{L}$ of $\mathrm{NaNO}_{2}$ was attributed to a change in $\mathrm{pH}$ close to the $\mathrm{pKa}$ of the adsorbates. At $50 \mathrm{mmoles} / \mathrm{L}$ adsorption increased for all of the salts due to decreased solubility. Deng et al. (2009) found that adsorption increased with increasing $\mathrm{NaCl}$ concentration until nearly all of the adsorbate was removed at a salt concentration of $1 \mathrm{~mol} / \mathrm{L}$. The difference between MIP and NIP also decreased at higher salt concentrations. This is probably because decreasing the solubility of the adsorbate would increase the non-specific binding. Zhang and $\mathrm{Hu}$ (2008) found no influence of ionic strength on MIP for adsorption of estrone (E1), $17 \beta$-estradiol (E2), or bisphenol A (BPA), but increased adsorption of EE2, which was the most hydrophobic compound studied. 


\subsection{Effect of competing micropollutants}

Although MIP are designed with one template molecule, similar molecules are also capable of binding into the specific cavity, and the template experiences some competition from these similar compounds. Both MIP and NIP also exhibit some degree of non-specific adsorption, which is not compound-specific.

Yu et al. (2008) and Deng et al. (2009) both studied PFOS imprinted MIP for adsorption of PFOS in solution. Yu et al. (2008) studied a solution of PFOS with an equal concentration of phenol, sodium dodecyl benzene sulfonate, or pentachlorophenol. They observed decreased adsorption of PFOS, but the adsorption decreased less for MIP than NIP. Deng et al. (2009) investigated the competition from 2,4 Dichlorophenoxyacetic acid (2,4-D). They found good selectivity and adsorption decreased less than $5 \%$ for an increase in 2,4-D concentration from $0-2.26 \mathrm{~mol} / \mathrm{L}$.

Li et al. (2009) studied adsorption with 2,4-D imprinted MIP. They tested adsorption of 2,4-D in the presence of methylbenzene and aniline which have similar structures to 2,4D. Adsorption decreased from $83.2 \%$ to approximately $72 \%$, but adsorption of the competitors was below $40 \%$. Higher adsorption of the template molecule in the presence of other competing molecules is indicative of good selectivity.

\subsection{Effect of water and wastewater constituents}


Since MIP have both specific and non-specific adsorption sites, they should theoretically have some decrease in capacity in the presence of contaminants found in real water or wastewater, but should retain capacity to adsorb the template in the cavity.

Krupadam et al. (2010) collected groundwater from industrial areas in India and spiked it with a mixture of PAH compounds to a total concentration of $1.2 \mathrm{mg} / \mathrm{mL}$. The groundwater was mixed in different ratios with triple distilled water to determine the effect of changing total dissolved solids (TDS) and chemical oxygen demand (COD) on MIP performance. They found that the capacity of the MIP did not decrease until after 50\% dilution which represented $690 \mathrm{mg} / \mathrm{L}$ TDS and $430 \mathrm{mg} / \mathrm{L}$ of COD. The decrease at this concentration was attributed to a change in $\mathrm{pH}$ caused by the increased TDS concentration and not by competition for adsorption sites from the COD or TDS.

Lin et al. (2008) studied the effect of humic acid on the efficiency of MIP for removal of phenolic estrogens. They found no decrease for up to $10 \mathrm{mg} / \mathrm{L}$ of humic acid. This was because the monomer (4-vindylpyridine) did not have an interaction with the humic acid. Also, humic acid decreased the $\mathrm{pH}$ which increased removal. They also tested removal with $20 \mathrm{~mL}$ of spiked triple distilled water, tap water, river water, and lake water and found removal efficiencies increasing in the order listed.

Le Noir et al. (2007a) collected $100 \mathrm{~mL}$ wastewater samples following primary treatment and percolated them through SPE columns prepared with MIP. They then eluted the columns and measured the estrogenic activity of the eluate. MIP extracts showed the 
equivalent of $22 \pm 4 \mathrm{ng}$ E2/L of water. Luo et al. (2011) tested spiked wastewater for removal of five acidic dyes. They found a high efficiency between $91.7-97.4 \%$ for removal of the five dyes, and suggested that the dyes could be recycled and reused from the wastewater.

\subsubsection{Application methods}

Because MIP are very small, a means of applying and removing the particles is necessary before they can be used for treatment applications. Two options have been considered for this purpose: the creation and incorporation of the particles into a macroporous gel, and the incorporation of a magnetic core into the MIP.

Le Noir et al. (2007b) first created a macroporous gel with MIP particles incorporated into the gel structure for the purpose of wastewater treatment. They compared the use of the gel to a column of MIP particles and found that the gel was capable of processing a much larger volume. The gel columns could operate at a flowrate of $50 \mathrm{~mL} /$ minute to provide complete recovery of E2 from a $2 \mu \mathrm{g} / \mathrm{L}$ solution. The MIP particle column could operate at a flow rate of only 1 to $5 \mathrm{~mL} /$ minute and could only treat $55 \mathrm{~mL}$ of water at $1 \mathrm{~mL} / \mathrm{minute}$ before clogging. Le Noir et al. (2009) then created a PVA cryogel with embedded MIP which was formed inside of plastic Kaldenes carriers. Packed and moving-bed configurations were compared and the moving-bed configuration was shown to be superior due to increased mixing and improved contact between the MIP and solution. Hajizadeh et al. (2010) also created polyacrylamide monolithic composite cryogels with embedded MIP. They found that due to freezing during the creation of the cryogels, which forced the MIP 
particles outwards from the growing ice front, the MIP particles remained at the surface of the gel. This allowed for good access during water treatment.

Luo et al. (2011), Guo et al. (2011), and Xia et al. (2012) took another approach and prepared magnetic MIP. Luo et al. (2011) were interested in treating industrial wastewater for water soluble acidic dyes. They also wanted to recover and reuse the dye once it had been removed from the wastewater and achieved good results with magnetic particles. Guo et al. (2011) created magnetic polymers for separation of BPA from aqueous solutions. Their polymers contained a kaolinite $/ \mathrm{Fe}_{3} \mathrm{O}_{4}$ core which also acted as the monomer for polymerization. Xia et al. (2012) prepared molecularly imprinted polymer-encapsulated magnetic particles by ultrasonication assisted synthesis in a mixture of water and organic solvent using dual-layered surfactant-modified magnetic particles as core, $17 \beta$-estradiol as template, and methacrylic acid as functional monomer (Xia et al., 2013).

Xie et al. (2011) used MIP as a surface for adsorption of BPA and its metabolites to increase the concentration and aid biodegradation. Biodegradation was enhanced by the addition of MIP, NIP, or activated carbon, but the best results were achieved with MIP. The effect of antiseptic heavy metals such as $\mathrm{Hg}$ and $\mathrm{Ag}$ were also reduced in the presence of MIP, and although river water and lake water decreased biodegradation, adsorption was not affected for MIP as it was for NIP. Xie et al. (2011) also added MIP to activated sludge to improve the biodegradation of BPA. 
Shen et al. (2012) synthesized MIP-coated photocatalysts via an in-situ polymerization of o-phenylenediamine in the presence of target molecules and $\mathrm{TiO}_{2}$ nanoparticles, and the newly created hybrid photocatalysts were able to selectively remove low-concentration target pollutants in a mixture of high-concentration pollutants (Shen et al., 2012).

\subsubsection{Regeneration and reuse of MIP}

Regeneration and subsequent reuse of MIP following their use for water and wastewater treatment can reduce the overall costs of treatment. Several researchers have investigated reuse of MIP including the number of cycles the particles can be reused for (Meng et al., 2005; Krupadam et al., 2010; Hajizadeh et al., 2010; Meng et al., 2012; Lin et al., 2008; Luo et al., 2011; Yu et al., 2008; Li et al., 2009; Guo et al., 2011, Hassanzadeh-Khayyat et al., 2011) and treatment of concentrated micropollutants following regeneration (Le Noir et al., 2007a; Fernández-Alvarez et al., 2009; Lai et al., 2011). Meng et al. (2005) and Li et al. (2009) both regenerated MIP with methanol/acetic acid (9/1, v/v) and were able to reuse the particles for 5 cycles without a loss in efficiency. Lin et al. (2008) showed that their particles could be used for 30 cycles with no loss in efficiency. However, none of these researchers continued testing the regeneration of the particles until they observed a decrease in performance so it is unknown how many times their particles could have been regenerated.

Krupadam et al. (2010) did continue to test until they observed a decline and demonstrated that their particles could be used for 10 cycles before a decline in performance was observed after 15 cycles. Hajizadeh et al. (2010) were able to regenerate MIP created to 
remove bromate before they saw a decrease in performance, and Meng et al. (2012) observed a decrease in performance of $7.3 \%$ over 4 cycles, which was attributed to a loss of particles. Guo et al. (2011) tested their particles for 4 cycles with $85 \%$ and $79 \%$ readsorption in the third and fourth cycles. Although different MIP can be regenerated for different numbers of cycles, they all show good regeneration potential which can lead to decreased costs and decreased waste.

Le Noir et al. (2007a) demonstrated biological treatment of enriched E2 using 50mg/L of E2 in tubes inoculated with $0.5 \mathrm{~mL}$ of a pure suspension of the E2 degrading Noosphingobrium tardaugens. The E2 concentration was reduced by $85+/-3 \%$ after 14 days. This suggests using biodegradation under ideal conditions over a long time following enrichment of E2 from wastewater using MIP is a potential treatment method.

Fernández-Álvarez et al. (2009) developed a method for regeneration of MIP with an E2 template using solvent extraction under UV light. This allowed for the regeneration and reuse of both the MIP and the solvent as well as the destruction of E2. Acetone was used as a solvent under both UVC and UVvis light. Following a 10 hour cycle, the MIP were completely regenerated and the acetone contained no residual E2. Hassanzadeh-Khayyat et al. (2011) showed that diclofenac inside MIP degraded completely after exposure to UV irradiation and UV light could be used for regeneration.

Lai et al. (2011) tested hydrogen peroxide, hydrochloric acid, and nitric acid for degradation of diclofenac adsorbed to MIP. Nitric acid was the most efficient and the extent 
of degradation was dependent on the time and concentration of the degrading agent for all of the agents tested. Rebinding was not as successful as anticipated which may have been caused by degradation products remaining adsorbed on the MIP after treatment.

\subsubsection{Potential use of NIP for water and wastewater treatment}

Another idea to consider, which has not been considered in much depth to date, is the use of NIP for water and wastewater treatment (Murray et al., 2011). The main difference between MIP and NIP is their specificity. MIP can selectively target and remove a single compound (such as E2, EE2, etc.) whereas NIP can remove several organic compounds simultaneously. Though MIP generally show higher selectivity and stronger binding to specific chemicals than NIP, NIP may have a higher potential for general water and wastewater treatment applications due to their ability to remove several emerging contaminants simultaneously. NIP are also simpler, quicker, and less costly to prepare compared to MIP as they do not require the presence of templates and extensive washing steps for the removal of templates. Most importantly, NIP can be manufactured in large quantities and thus can be used for treatment of large flows. NIP particles appear to be a very promising tool as a polishing step to remove various emerging contaminants and improve the overall quality of treated wastewater.

Most researchers investigating the use of MIP for water treatment have inadvertently also tested NIP. NIP are frequently tested alongside MIP to show the strength of the imprinting effect and the difference in binding efficiencies between MIP and NIP is used as a measure of the strength and specificity of the specific adsorption sites on the MIP. Table 3-1 
provides some of the binding efficiencies for MIP and NIP measured by researchers investigating the use of MIP for water treatment. From the table, it is clear that there are a wide range of adsorption efficiencies and also a wide range of differences in adsorption efficiencies between MIP and NIP. In some cases the NIP had a higher adsorption capacity for the template than the MIP. MIP researchers have typically focused on creating MIP with the greatest specificity possible, and hence the greatest difference in performance from NIP. It may be possible to create NIP with an excellent adsorption capacity if the design of the NIP is optimized. 
Table 3-1: Binding efficiencies for MIP and NIP prepared with various methods

\begin{tabular}{|c|c|c|c|c|c|c|c|c|c|c|}
\hline \multirow[t]{2}{*}{ Reference } & \multicolumn{2}{|c|}{ Efficiency } & \multirow[t]{2}{*}{ Template } & \multirow[t]{2}{*}{ Monomer } & \multirow[t]{2}{*}{ Crosslinker } & \multirow{2}{*}{ Initiator } & \multirow{2}{*}{$\begin{array}{l}\mathrm{T} / \mathrm{U} \\
\mathrm{V}\end{array}$} & \multirow[t]{2}{*}{ Solvent } & \multirow[t]{2}{*}{ Sample type } & \multirow[t]{2}{*}{ Particles added } \\
\hline & MIP & NIP & & & & & & & & \\
\hline $\begin{array}{l}\text { Le Noir et al } \\
2009\end{array}$ & 100 & 77 & $\mathrm{E} 2^{1}$ & $\begin{array}{l}\mathrm{VP}^{2} \\
\mathrm{mmol})\end{array}$ & \multirow[t]{3}{*}{$\begin{array}{l}\mathrm{EGDMA}^{3} \\
(25 \mathrm{mmol})\end{array}$} & $\begin{array}{l}\mathrm{AIBN}^{4} \\
(50 \mathrm{mg})\end{array}$ & \multirow[t]{3}{*}{$\begin{array}{l}65^{\circ} \\
\mathrm{C}\end{array}$} & $\begin{array}{l}\mathrm{ACN}^{5} \\
(8 \mathrm{~mL})\end{array}$ & $0.5 \mathrm{ppb}$ E2 in water & \multirow[t]{3}{*}{$\begin{array}{l}\text { moving bed reactor } \\
\text { plastic carriers }\end{array}$} \\
\hline $\begin{array}{l}\text { Le Noir et al } \\
2009\end{array}$ & 76 & 69 & $\mathrm{NP}^{6}$ & $\begin{array}{l}\text { DEAEM }^{7}(5 \\
\text { mmol })\end{array}$ & & $\begin{array}{l}\text { AIBN } \\
(330 \mathrm{mg})\end{array}$ & & $\begin{array}{l}\mathrm{DMF}^{8} \quad(5.3 \\
\mathrm{mL})\end{array}$ & 5 ppb NP in water & \\
\hline $\begin{array}{l}\text { Le Noir et al } \\
2009\end{array}$ & 75 & 26 & Atrazine & $\begin{array}{l}\mathrm{MAA}^{9} \\
\mathrm{mmol})\end{array}$ & & $\begin{array}{l}\text { AIBN }(50 \\
\mathrm{mg})\end{array}$ & & $\begin{array}{l}\text { Toluene }(5 \\
\mathrm{mL})\end{array}$ & $\begin{array}{l}10 \mathrm{ppb} \text { atrazine in } \\
\text { water }\end{array}$ & \\
\hline $\begin{array}{l}\text { Zhang et al } \\
2010\end{array}$ & 116.3 & 98.1 & \multirow[t]{2}{*}{$\begin{array}{l}\text { E2 } \quad(50 \\
\mathrm{mg})\end{array}$} & \multirow[t]{2}{*}{$\begin{array}{l}\text { MAA } \quad(75 \\
\mathrm{mg})\end{array}$} & \multirow[t]{2}{*}{$\begin{array}{l}\text { TRIM }^{10} \\
(254.4 \mathrm{mg})\end{array}$} & \multirow[t]{2}{*}{$\begin{array}{l}\mathrm{ACVA}^{11} \\
(2.0 \mathrm{mg})\end{array}$} & \multirow[t]{2}{*}{ UV } & $\mathrm{ACN}$ & \multirow[t]{2}{*}{$4 \mu \mathrm{M}$ E2 in water } & \multirow[t]{2}{*}{$0.05 \mathrm{mg} / \mathrm{mL}$} \\
\hline $\begin{array}{l}\text { Zhang et al } \\
2008\end{array}$ & 95.8 & 80 & & & & & & $\begin{array}{l}\mathrm{MeCN}^{12} \\
(10 \mathrm{~mL})\end{array}$ & & \\
\hline $\begin{array}{l}\text { Hajizadeh et al } \\
2010\end{array}$ & 70 & 30 & Bromate & chitosan & $\mathrm{ECH}^{13}$ & N/A & $\begin{array}{l}40^{\circ} \\
\mathrm{C}\end{array}$ & acetic acid & $\begin{array}{l}30 \mu \mathrm{g} / \mathrm{L} \text { bromate in } \\
\text { water }\end{array}$ & $0.01 \mathrm{~g} / 100 \mathrm{~mL}$ \\
\hline Luo et al 2011 & $90-98$ & & Tratarzine & $\begin{array}{l}\text { 1-MA-3MI- } \\
\mathrm{Br}^{14}\end{array}$ & $\begin{array}{l}\text { TRIM } \quad(10 \\
\mathrm{mL})\end{array}$ & $\begin{array}{l}\text { AIBN } \\
(0.1 \mathrm{~g})\end{array}$ & $\begin{array}{l}70^{\circ} \\
\mathrm{C}\end{array}$ & $\begin{array}{l}\text { toluene } \quad(2 \\
\mathrm{mL})\end{array}$ & $\begin{array}{l}100 \text { ppm aq. mix of } 5 \\
\text { dyes }\end{array}$ & $2 \mathrm{~g} / 100 \mathrm{~mL}$ \\
\hline $\begin{array}{l}\text { Le Noir et al } \\
2007^{\mathrm{b}}\end{array}$ & 100 & 77 & E2 & $\begin{array}{ll}\mathrm{VP} & (0.430 \\
\mathrm{mL}) & \\
\end{array}$ & $\begin{array}{l}\text { EGDMA } \\
4.72 \mathrm{~mL}\end{array}$ & $\begin{array}{l}\text { AIBN } \\
0.05 \mathrm{~g}\end{array}$ & $\begin{array}{l}65 \\
{ }^{\circ} \mathrm{C} \\
\end{array}$ & $\mathrm{ACN}(8 \mathrm{~mL})$ & 2L 2 ppb E2 in water & $100 \mathrm{mg}$ columns \\
\hline $\begin{array}{l}\text { Krupadam et al } \\
2010\end{array}$ & 80 & 10 & $6 \mathrm{PAHs}^{15}$ & $\begin{array}{l}\text { MAA } \\
(3.44 \mathrm{mg})\end{array}$ & $\begin{array}{l}\text { EGDMA } \\
(151.02 \mathrm{mg})\end{array}$ & $\begin{array}{l}\text { AIBN } \quad(2 \\
\mathrm{mg})\end{array}$ & $\begin{array}{l}\mathrm{Hg} \\
\text { lam } \\
\mathrm{p}\end{array}$ & $\begin{array}{l}\mathrm{ACN} \\
\mathrm{mL})\end{array}$ & $\begin{array}{l}1.2 \mathrm{mg} / \mathrm{mL} \text { mix of } 6 \\
\text { PAH's in water }\end{array}$ & $\mathrm{N} / \mathrm{A}$ \\
\hline $\begin{array}{l}\text { Meng et al } \\
2005\end{array}$ & 80 & 10 & $\alpha$-estradiol & $\begin{array}{l}\mathrm{AM}^{16} \\
(3 \mathrm{mmol})\end{array}$ & $\begin{array}{l}\text { TRIM } \quad(3 \\
\text { mmol })\end{array}$ & $\mathrm{N} / \mathrm{A}$ & UV & $\begin{array}{l}\mathrm{MeCN} \quad(40 \\
\mathrm{mL})\end{array}$ & $1 \mathrm{~mL} 0.1 \mathrm{mM}$ water & $1 \mathrm{mg} / \mathrm{mL}$ \\
\hline Lin et al 2008 & 85.46 & 56.4 & $\mathrm{BPA}^{17}$ & $\begin{array}{l}\text { 4-VP } \\
(6 \mathrm{mmol})\end{array}$ & $\begin{array}{l}\text { TRIM } \quad(12 \\
\text { mmol })\end{array}$ & $\begin{array}{l}\text { AIBN } \\
(40 \mathrm{mg})\end{array}$ & $\begin{array}{l}65^{\circ} \\
\mathrm{C}\end{array}$ & & $\begin{array}{l}0.5 \mathrm{mmol} / \mathrm{L} \text { of } \mathrm{BPA} \\
\text { in water }\end{array}$ & $100 \mathrm{mg}$ cartridges to trea \\
\hline Xie et al 2011 & $\begin{array}{l}5.33 \\
(\mathrm{mg} / \mathrm{g} \\
)\end{array}$ & $\begin{array}{l}0.96 \\
(\mathrm{mg} / \mathrm{g})\end{array}$ & $\begin{array}{l}\text { BPA } \\
(6 \mathrm{mmol})\end{array}$ & $\begin{array}{l}4-\mathrm{VP} \\
(6 \mathrm{mmol})\end{array}$ & $\begin{array}{l}\text { TRIM } \quad(12 \\
\text { mmol })\end{array}$ & $\begin{array}{l}\text { AIBN } \\
(40 \mathrm{mg})\end{array}$ & $\begin{array}{l}65^{\circ} \\
\mathrm{C}\end{array}$ & $\begin{array}{l}\mathrm{ACN} \\
(250 \mathrm{~mL})\end{array}$ & $\begin{array}{l}22.8-1140 \quad \mathrm{mg} / \mathrm{L} \\
\text { BPA in ACN }\end{array}$ & $10 \mathrm{mg}$ \\
\hline $\begin{array}{l}\text { Deng et al } \\
2009\end{array}$ & 60 & 40 & $\begin{array}{l}\text { PFOS }^{18} \\
(80 \mathrm{mM})\end{array}$ & $\begin{array}{ll}4-\mathrm{VP} & (320 \\
\mathrm{mM}) & \\
\end{array}$ & $\begin{array}{l}\text { TRIA }^{19} \\
(1.6 \mathrm{~mol} / \mathrm{L})\end{array}$ & AIBN & $\begin{array}{l}65^{\circ} \\
\mathrm{C}\end{array}$ & $\begin{array}{l}\text { acetone/Me } \\
\mathrm{OH}^{20}\end{array}$ & $200 \mathrm{mg} / \mathrm{L}$ PFOS & $0.02 \mathrm{~g}$ for $4 \mathrm{~h}$ \\
\hline $\begin{array}{l}\text { Deng et al } \\
2009\end{array}$ & 45 & 45 & $\begin{array}{l}\text { PFOS }(10 \\
\mathrm{mM})\end{array}$ & $\begin{array}{ll}4-\mathrm{VP} & (10 \\
\mathrm{mM}) & \end{array}$ & $\begin{array}{l}\text { TRIA } \\
\mathrm{mmol} / \mathrm{L})\end{array}$ & & & $\begin{array}{l}\text { acetone/Me } \\
\mathrm{OH}\end{array}$ & $200 \mathrm{mg} / \mathrm{L}$ PFOS & $0.02 \mathrm{~g}$ for $4 \mathrm{~h}$ \\
\hline $\begin{array}{l}\text { Meng et al } \\
2012\end{array}$ & 92.2 & 40.7 & & $\mathrm{AM}(3 \mathrm{mmol})$ & $\begin{array}{l}\text { EDGMA (15 } \\
\text { mmol) }\end{array}$ & & UV & $\begin{array}{l}\mathrm{ACN} \\
(40 \mathrm{~mL})\end{array}$ & & N/A \\
\hline
\end{tabular}




\begin{tabular}{|c|c|c|c|c|c|c|c|c|}
\hline $\begin{array}{l}\text { Meng et al } \\
2012\end{array}$ & 86.9 & 83.5 & \multirow{11}{*}{$\begin{array}{l}\mathrm{TNT}^{21} \\
(0.5 \\
\mathrm{mmol})\end{array}$} & & & \multirow{11}{*}{$\begin{array}{l}\text { AIBN } \\
(0.035 \\
\text { mmol })\end{array}$} & $\begin{array}{l}\mathrm{MeOH} \\
(40 \mathrm{~mL})\end{array}$ & \multirow{11}{*}{$\begin{array}{l}90 \% \text { TNT solution in } \\
\text { water/MeOH }(90 / 10 \\
\text { v/v) }\end{array}$} \\
\hline $\begin{array}{l}\text { Meng et al } \\
2012\end{array}$ & 88.1 & 50 & & $\begin{array}{l}\mathrm{AA}^{22} \\
\mathrm{mmol})\end{array}$ & & & $\begin{array}{l}\mathrm{ACN} \\
(40 \mathrm{~mL})\end{array}$ & \\
\hline $\begin{array}{l}\text { Meng et al } \\
2012\end{array}$ & 88.5 & 91.2 & & $\begin{array}{l}\mathrm{IA}^{231} \\
(2 \mathrm{mmol})\end{array}$ & \multirow[t]{3}{*}{$\begin{array}{ll}\text { EDMA } & (10 \\
\text { mmol }) & \end{array}$} & & & \\
\hline $\begin{array}{l}\text { Meng et al } \\
2012\end{array}$ & 84.7 & 56.1 & & \multirow[t]{2}{*}{ N/A } & & & & \\
\hline $\begin{array}{l}\text { Meng et al } \\
2012\end{array}$ & 89.5 & 91.1 & & & & & $\begin{array}{l}\mathrm{MeOH} \\
(40 \mathrm{~mL})\end{array}$ & \\
\hline $\begin{array}{l}\text { Meng et al } \\
2012\end{array}$ & 82.5 & 82.8 & & \multirow[t]{2}{*}{$\mathrm{AM}(3 \mathrm{mmol})$} & \multirow[t]{3}{*}{$\begin{array}{ll}\text { TRIM } & (3 \\
\text { mmol }) & \end{array}$} & & $\begin{array}{l}\mathrm{ACN} \\
(40 \mathrm{~mL})\end{array}$ & \\
\hline $\begin{array}{l}\text { Meng et al } \\
2012\end{array}$ & 88.7 & 45 & & & & & $\begin{array}{l}\mathrm{MeOH} \\
(40 \mathrm{~mL})\end{array}$ & \\
\hline $\begin{array}{lll}\text { Meng } & \text { et al } \\
2012 & & \\
\end{array}$ & 81.4 & 56.2 & & $\begin{array}{ll}\begin{array}{l}\text { AA } \\
\text { mmol })\end{array} & (3 \\
\end{array}$ & & & $\begin{array}{l}\mathrm{ACN} \\
(40 \mathrm{~mL})\end{array}$ & \\
\hline $\begin{array}{l}\text { Meng et al } \\
2012\end{array}$ & 92.5 & 90.2 & & IA $(2 \mathrm{mmol})$ & \multirow[t]{3}{*}{$\begin{array}{ll}\text { TRIM } & (2 \\
\text { mmol }) & \end{array}$} & & & \\
\hline $\begin{array}{lll}\text { Meng et al } \\
2012\end{array}$ & 85.8 & 94.5 & & \multirow[t]{2}{*}{$\mathrm{N} / \mathrm{A}$} & & & & \\
\hline $\begin{array}{l}\text { Meng et al } \\
2012\end{array}$ & 90.3 & 95.5 & & & & & $\begin{array}{l}\mathrm{MeOH} \\
(40 \mathrm{~mL})\end{array}$ & \\
\hline
\end{tabular}

${ }^{1} 17 \beta$-estradiol ${ }^{2}$ 4-vinylpyridine ${ }^{3}$ Ethylene glycol dimethacrylate ${ }^{4} 2,2$-azobisisobutyronitrile ${ }^{5}$ Acetonitrile ${ }^{6}$ Nonylphenol ${ }^{7}$ Diehtylaminoethyl methacrylate ${ }^{8}$ Dimethylformamide ${ }^{9}$ Methacrylic acid ${ }^{10}$ Trimethacrylate ${ }^{11} 4,4^{\prime}$-azobis(4- cyanovaleric acid) ${ }^{12}$ Anhydrous acetonitrile ${ }^{13}$ Epichlorohydrin ${ }^{14}$ 1-( $\alpha$-methyl acrylate)-3-methylimidazolium bromide ${ }^{15}$ Polyaromatic hydrocarbon ${ }^{16}$ Acrylamide ${ }^{17}$ Bisphenol A ${ }^{18}$ Perfluorooctanesulfonic acid ${ }^{19}$ Trimethylolpropane triacrylate ${ }^{20}$ Methanol ${ }^{21}$ Trinitrotoluene ${ }^{22}$ Acrylic Acid ${ }^{23}$ Iticonic acid 


\subsubsection{Physical differences between MIP and NIP}

There are some physical differences between MIP and NIP which may account for the differences in adsorption efficiencies. Further investigation of these physical differences may yield information about how to improve the adsorption capacity of NIP.

Investigations into the physical differences are typically used to confirm imprinting of the MIP. MIP are generally more porous and contain visible imprinting cavities (Le Noir et al., 2007a; Krupadam et al., 2010; Zhang and Hu, 2009). Some researchers have found that this leads to an increased surface area (Le Noir et al., 2007a; Krupadam et al., 2010; Zhang and $\mathrm{Hu}, 2009$; Randhawa et al., 2007) and others have not (Luo et al., 2011). In the cases where the surface area is greater for MIP than NIP, it may be possible to attribute increased binding efficiency of MIP to their larger surface areas.

Randhawa et al. (2007) and Ashraf et al. (2011) created both porous and nonporous MIP and NIP. The porous particles were created by bubbling nitrogen gas into the reaction mixture during polymerization. The porous MIP and NIP both showed better binding of heavy metals confirming the hypothesis that porosity enhances adsorption.

\subsubsection{Selectivity in aqueous environments}

One of the reasons why many of the researchers using MIP for water treatment did not notice a large difference in binding efficiencies between MIP and NIP is that selectivity of MIP in aqueous environments tends to be low. Since NIP do not have templates, this would not be an issue for NIP. 
Selectivity of MIP is dependent on both the presence of matching and interacting sites and their orientation. In different mediums, MIP have different swelling properties and their orientations can change. Polar solvents, such as water, are not used during polymerization of noncovalent MIP because they are capable of forming hydrogen bonds, which reduce the formation of imprinted sites (Spivak, 2005).

This problem has been addressed for the use of MIP for solid phase extraction. In this case, a buffer or an additive can be used to aid binding. For example, Karlsson et al. (2001) found that for a buffered solution, their MIP, which were prepared with a bupivacaine template, bound preferentially to bupivacaine although binding for other molecules was favoured according to hydrophobicity. In a solution of $20 \%$ acetonitrile in water with no buffer, however, pentycaine was favoured over the template. This work suggests that without the use of a buffer, MIP may not be capable of maintaining their selectivity although this would be dependent on the MIP used and their swelling properties in different mediums. That said, MIP have been used for water treatment with selectivity largely dependent on the MIP used. For example, Le Noir et al. (2009), using two different MIP and NIP for NP and E2, found a difference of $7 \%$ for NP and $23 \%$ for E2.

\subsubsection{Effect of water and wastewater constituents on NIP}

One of the justifications behind using MIP for water and wastewater treatment is that since MIP are specific to a target compound, even in wastewater with many competing substances, MIP would be capable of removing the target compound alone. NIP are not specific to a target compound so they do not have this advantage; but they can still remove 
the target compound together with other compounds. For a target compound, the difference in adsorption capacity between MIP and NIP could be investigated in the water to be treated to determine which is more cost effective. For cases of municipal water or wastewater treatment, NIP may be desirable because they have the ability of removing a variety of contaminants simultaneously. On the other hand, MIP which are designed to have high selectivity and strong affinity for target chemicals may be ideal for certain applications, such as industrial wastewater treatment, where high concentrations of specific compounds used in manufacturing need to be removed from wastewater.

Murray et al. (2009) compared MIP and NIP for treatment of E2 and EE2. For E2, NIP performed equivalent to or slightly better than MIP for 0.01-0.05 mg E2/ g particles. For EE2, the advantage of NIP was even more pronounced because MIP exhibited negative removal efficiencies for many of the concentrations tested. The negative removal efficiencies were caused by template bleeding from the MIP cavities. Template bleeding occurs when the template is not completely removed during MIP synthesis and later leaches out into the solution being treated. This is a potential problem for drinking water treatment where leaching of the template could be hazardous.

Murray et al. (2011) studied the use of NIP for wastewater treatment. Secondary wastewater effluent was spiked with $10 \mathrm{mg} / \mathrm{L}$ of E2 and the NIP effectively removed $98 \%$ of the E2. The concentrations of both the E2 $(10 \mathrm{mg} / \mathrm{L})$ and the NIP $(15 \mathrm{mg} / \mathrm{mL})$ were high in this case, but it illustrated that NIP could successfully remove even very high concentrations of E2. The work also illustrated that additional NIP led to additional 
removal. For example, addition of $5 \mathrm{mg} / \mathrm{mL}$ of NIP removed $89.8 \%$ of E2 from wastewater.

Xie et al. (2011) tested the addition of MIP, NIP, and activated carbon along with activated sludge for treatment of river water and lake water. Note that the activated sludge was added to the surface water samples to study the effects of simultaneous adsorption and biodegradation. There was no decrease in adsorption for the MIP, and NIP did exhibit a decrease in performance in comparison to deionized water, but the decrease was less than that of activated carbon. This illustrates that NIP can be used to effectively remove BPA from water and wastewater.

\subsubsection{Application of NIP for water and wastewater treatment}

While the specificity of MIP is an advantage for solid phase-extraction, the same may not be true for water and wastewater treatment applications. For water and wastewater treatment, it may be preferable to remove a wide range of contaminants simultaneously using NIP which are nonspecific and simpler to prepare compared to MIPs.

Murray et al. (2009) investigated the removal of atrazine, BPA, and diethylstilbestrol (DES) with NIP prepared for the removal of E2. For the range of 1-5 mg E2/ g NIP, the removal efficiencies were $50-70 \%, 91-97 \%$, and $25-45 \%$, for atrazine, BPA, and DES, respectively. The NIP were also capable of reducing the ammonia, total organic carbon, and chemical oxygen demand concentrations from a wastewater sample, although the capability of the NIP to remove spiked E2 from the same wastewater sample was 
equivalent to a deionized water sample. The ability of NIP to remove both micropollutants as well as other pollutants from wastewater could be a major advantage for treatment applications.

Murray et al. (2011) used a combination of chlorination followed by application of NIP to remove E2 as well as the chlorination by-products of E2. Chlorination can be used to oxidize E2, but can result in the formation of by-products. By applying NIP following chlorination, it was possible to remove both the remaining E2 by greater than $99 \%$ and the chlorination by products by $85 \%$. Removal of $\mathrm{E} 2$ and the by-products was not affected by the addition of $5 \mathrm{mg} / \mathrm{L}$ of humic acid.

\subsection{HEAVY METALS AND ION-IMPRINTED POLYMERS}

\subsubsection{Wastewater as a source of heavy metal contamination}

Wastewater effluents are a major source of heavy metals in the environment and in the food chain. Wastewater effluents are the largest source of heavy metals in rivers and lakes (Ryan et al., 1982) and, due to increasing water stress, re-use of wastewater effluent for irrigation is a major contributor to heavy metals in the food supply (Mapanda et al., 2005; Wang et al., 2005; Toze, 2006; Arora et al., 2008; Singh et al., 2010). Sigh et al (2010) compared concentrations of heavy metals in agricultural fields in India irrigated with wastewaters to fields which had not been irrigated with wastewater and found that those irrigated with wastewater had heavy metal concentrations several times those in fields not irrigated with wastewater. They found vegetables and cereals with concentrations of cadmium, lead, and nickel above Indian limits and those set by the World Health 
Organization. Other researchers (Mapanda et al., 2005; Wang et al., 2005; Arora et al., 2008), working in other areas, measured food concentrations below permissible limits, but measurements focused on a single food source may not accurately reflect heavy metal intake, which is cumulative and comes from many dietary sources (Wang et al., 2005). Mapanda et al (2005) also found that concentrations of heavy metals in soils increased with time for fields irrigated with wastewater over many years. They studied fields that had been irrigated with wastewater for more than 10 years and predicted that within 5 to 60 years all of the locations they studied would have heavy metal concentrations above permitted limits (Mapanda et al., 2005). High concentrations of heavy metals in agricultural soils can decrease crop yields and quality, enter the food chain, or leach into the groundwater used as drinking water resources (Senesi et al., 1999).

\subsubsection{Heavy metal effects on crops and agriculture}

Some heavy metals such copper, manganese, cobalt, zinc, and chromium are required by plants in trace amounts, but excessive amounts, or the presence of other heavy metals, can be detrimental to crops. Plant uptake of high concentrations of heavy metals from agricultural soils can reduce safety and marketability of crops (Nagajyoti et al., 2010). In

plants, heavy metals can interfere with photosynthesis, exchange of gases, and nutrient adsorption. This causes reduced plant growth, leading to lower yield, and also lowers antioxidant and nutrient concentrations in plants (Sharma and Agrawal, 2005). 


\subsubsection{Human health effects of heavy metals}

Heavy metals cause numerous adverse health effects in humans. Heavy metals can accumulate in and affect the function of the heart, brain, kidneys, bone, liver, and other vital organs or they can displace essential nutrients required by the body (Singh et al., 2011). Cadmium, lead, and mercury are the most prevalent of the toxic heavy metals. They have a cumulative effect and can be toxic at low doses (Chowdhury, 1987). The safe daily intake of mercury, for example, is $0.1 \mu \mathrm{g} / \mathrm{kg} /$ day, but mercury cannot be excreted by the human body, so the average 70kg person contains a total of $13 \mathrm{mg}$ of mercury (Houston, 2011). Cadmium, lead, and mercury have been shown to interact with calcium, zinc, iron, selenium, copper, chromium, and manganese, all of which are essential dietary nutrients (Chowdhury, 1987).

Mercury is the most dangerous heavy metal. It lowers antioxidant capacity, increases production of free radicals, increases inflammation, and causes immune dysfunction, cardiovascular disease and hypertension (Houston, 2011). Mercury can also cause lung or kidney damage or neurological and psychological symptoms (Jarup, 2003). Cadmium concentrates in the kidney, liver and pancreas, (Houston, 2011) causing kidney damage and renal failure (Jarup, 2003). It can also cause bone and skeletal damage and is a possible human carcinogen (Jarup, 2003). In animal studies, cadmium has been shown to reduce cardiac output, alter cardiac function, and increase cholesterol and blood pressure (Houston, 2011). Lead can cause headaches, irritability, or neurological symptoms as well as behavioural disturbances in children (Jarup, 2003). 


\subsubsection{Ion-imprinted polymers}

\subsubsection{Ion-imprinted polymers vs. MIP}

Like molecularly imprinted polymers (MIP), ion imprinted polymers (IIP) possess a preference for a template molecule. The difference is that MIP are prepared to target a molecule, and IIP are designed to target heavy metal ions. The design of IIP differs from that of MIP, but their function is the same. Unlike MIP, IIP require chelating functions to form multiple interactions with a single heavy metal ion (Rao et al., 2006; Branger et al., 2013). Chelating agents contain two or more electron donors which are capable of interacting with a polyvalent cationic metal ion (Chelating Agents in Medicine, 1971).

\subsubsection{Design of IIP}

Like MIP, there are several approaches for the design of IIP, but these approaches are all based on ion pairing between chelating agents incorporated into the polymer, and heavy metal ions. Therefore, IIP are most similar to MIP prepared via the non-covalent approach described in Section 3.1.2.2 above (Rao et al., 2006). The differences between approaches used for design of IIP are due to different methods used to incorporate chelating agents into the polymer structure, and not the type of interactions between the template and polymer. There are three approaches typically used to create IIP: (1) use of a polymerizable ligand (2) trapping of a ligand inside the polymer cavity, and (3) surface imprinting (Rao et al., 2006; Branger et al., 2013). Each of these approaches are discussed in detail below.

\subsection{Use of a polymerizable ligand}


The first method for creation of IIP uses a monomer (typically containing a vinyl group) which is also capable of functioning as a ligand (Branger et al., 2013). Polymerizable ligands can be cross-linked with monomers such as vinyl pyridine to form the polymer (Rao et al., 2006). This process is the most similar to MIP synthesis; polymerization occurs with a monomer and cross-linker in the presence of a template which is removed following polymerization (Branger et al., 2013). One disadvantage is that ligands have complex structures and it can be difficult to incorporate polymerizable functional groups into their structure (Branger et al., 2013).

\subsection{Trapping}

The second method used to create IIP uses a vinylated chelating agent, incorporated into the polymer structure like the first method described, but also a nonvinylated chelating agent, which becomes trapped inside the polymer cavity. Because there are limits to the types of vinylated chelating agents that can be created, the use of the second, trapped, chelating agent increases flexibility (Rao et al., 2006). A disadvantage is that the trapped chelating agent can sometimes bleed out of the template cavity during template removal (Branger et al., 2013).

\subsection{Surface imprinting}

Surface imprinting is used to create IIP with cavities at or near the surface of the polymer, which enhances template removal and cavity accessibility and increases the rate of binding (Branger et al., 2013). An amphiphilic monomer is used in an emulsion and imprinting 
occurs at the emulsion interface, which encourages cavities to form at the polymer surface (Rao et al., 2006).

\subsubsection{Specific and non-specific interactions}

Charged IIP resins can act non-specifically to adsorb heavy metal ions from solution using an ion exchange mechanism. IIP also contain template-specific adsorption cavities which preferentially adsorb the target ion due to the spatial arrangement of metal-ligand bonds (Ashraf et al., 2011). Non imprinted polymers, do not contain the template-specific adsorption cavities possessed by IIP, but they can still act non-specifically to adsorb heavy metal ions by ion exchange. Therefore, NIP may be able to remove heavy metal ions during water and wastewater treatment.

\subsection{COMPETITION WITH NATURAL ORGANIC MATTER (NOM)}

Natural organic matter (NOM), present in natural waters and wastewater, has been shown to reduce the adsorption capacity of activated carbon for micropollutants (Newcombe and Drikas, 1997; Pelekani and Snoeyink, 1999; Ebie et al., 2001; Newcombe et al., 2002; Matsui et al., 2003; Rossner et al., 2009). Activated carbon can experience reductions in adsorption capacity of several orders of magnitude. Reductions in the rate of adsorption are also common (Pelekani and Snoeyink, 1999) and, for full-scale treatment, reductions in adsorption kinetics can lead to decreased micropollutant removal because adsorption is rarely given time to reach equilibrium. Some researchers (Matsui et al., 2003; Rossner et al., 2009) found that adsorption of micropollutants in the presence of NOM was 
independent of the initial micropollutant concentration, sometimes over ranges of several orders of magnitude (Rossner et al., 2009).

\subsubsection{Mechanisms of competition}

NOM can reduce adsorption of micropollutants on activated carbon by two mechanisms:

(1) direct competition for adsorption sites, and (2) by blocking pores (Pelekani and Snoeyink, 1999; Pelekani and Snoeyink, 2000; Pelekani and Snoeyink, 2001; Li et al., 2003; Quinlivan et al., 2005). The pore size distribution of the activated carbon and the size distribution of the NOM in competition with the micropollutant, as well as the molecular size of the micropollutant, all play a role in the extent of the capacity reduction as well as the relative effects of direct competition and pore blocking. Micropollutants are generally smaller in size than NOM, and preferentially adsorb into pores similar to their molecular size (Ebie et al., 2001). Trace organic compounds typically adsorb into pores less than $15 \AA$ in diameter (Ebie, 1995; Pelekani and Snoeyink, 2000; Pelekani and Snoeyink, 2001; Quinlivan et al., 2005; Redding and Cannon, 2014). The ideal pore size differs for different micropollutants. For example, Quinlivan et al (2005), found that trichloroethylene (TCE) preferentially adsorbed into pores 7-9 $\AA$ in diameter while methyltert-butyl ether (MTBE) preferred those 8-11 $\AA$ in diameter (Quinlivan et al., 2005), but, in general, micropollutants adsorb into activated carbon micropores (Redding and Cannon, 2014). NOM, on the other hand, was found to adsorb into larger pores $15-50 \AA \AA$ in diameter (Ding et al., 2008), or greater (Ebie, 1995), depending on the type and size distribution of the NOM. Smaller NOM molecules, close in size to the micropollutant molecules, are capable of accessing the same pores, and competing directly for adsorption sites, whereas 
larger NOM molecules adsorb into smaller mesopores leading to the micropores and block, or partially block, the entrance to the mesopores (Quinlivan et al., 2005).

Redding et al (2014) found that MTBE adsorption correlated with the occurrence of very small micropores less than or equal to $4.06 \AA$ in diameter, but only if the activated carbon also contained pores $22-59 \AA$ in diameter. They concluded that the MTBE had to be able to pass through the mesopores to access the micropores and the interaction between the mesopores and micropores was important (Redding and Cannon, 2014). Increasing mesopores in the ideal range for NOM adsorption, increases micropollutant adsorption by reducing pore blocking (Ebie et al., 2001).

\subsubsection{Determination of the mechanisms}

Adsorption mechanisms are complicated and are influenced by electrostatic forces, pore volume distribution, and chemical interactions between the adsorbent and adsorbate as well as adsorbate-adsorbate interactions (Bjelopavlic et al., 1999). Thus distinguishing the mechanism by which a competition with NOM occurs can be difficult. Generally, researchers have studied changes in adsorption capacity between simultaneous loading and pre-loading, as well as changes in adsorption kinetics and the adsorption isotherms. During pre-loading, the adsorbent is exposed to NOM or natural waters and then to the adsorbate.

\subsubsection{Difference between simultaneous and pre-loading with NOM}

A comparison between adsorption capacity for simultaneous loading and pre-loading of activated carbon can be used to determine whether or not pore blocking is an issue for a 
specific activated carbon and water sample (Pelekani and Snoeyink, 1999; Pelekani and Snoeyink, 2000; Pelekani and Snoeyink, 2001). A decrease in adsorption between simultaneously loaded activated carbon and pre-loading is indicative of pore blocking (Pelekani and Snoeyink, 1999). During simultaneous loading, micropollutants and NOM both reach their final adsorption sites at similar times (Pelekani and Snoeyink, 1999), because, although micropollutant molecules are smaller and thus diffuse faster, they also travel further into the porous network of the activated carbon (Newcombe et al., 2002). When activated carbons are pre-loaded, pores are blocked with NOM before they are exposed to the micropollutant and thus the micropollutant does not have a chance to enter pores before they are blocked (Pelekani and Snoeyink, 1999).

\subsubsection{Adsorption kinetics}

Partially-blocked pores continue to allow for the diffusion of micropollutants, but decrease the rate of diffusion and thus the kinetics of adsorption (Matsui et al., 2003).

\subsubsection{Adsorption isotherms}

For a Freundlich adsorption isotherm, the slope is indicative of site heterogeneity. If the adsorption capacity changes, but the slope of the isotherm does not change, this means that the composition of small and large pores (high and low energy adsorption sites) has not changed, which indicates pore blocking. Direct site competition occurs primarily in small, high energy pores, and a reduction in the availability of small pores without a reduction in large pores, changes the slope of the isotherm (Pelekani and Snoeyink, 1999). 


\subsection{CONCLUSIONS}

The use of MIP and NIP for the removal of emerging compounds is a novel approach, and has the potential to achieve nearly complete removal of micropollutants from water and wastewater. The technology is based on the adsorption of micropollutants on nano or submicron size polymeric particles, and therefore does not result in the generation of oxidation or degradation by-products that can potentially be more harmful than the parent compound.

Based on the extensive literature review presented above, the following opportunities and research gaps were identified for the use of MIP and NIP.

MIP and NIP have a particularly high potential for wastewater treatment applications. MIP, which are designed to have high selectivity and strong affinity for target chemicals may be ideal for industrial wastewater treatment where high concentrations of specific compounds used in manufacturing need to be removed from wastewater. NIP, on the other hand, have potential for general water and wastewater treatment applications, which typically require the removal of various chemical contaminants simultaneously. Further work is required to investigate the ability of NIP to remove a wide range of emerging compounds, because past work has focused on use of MIP and has only inadvertently looked at removal with NIP.

In addition to containing emerging contaminants of concern, wastewater effluents are also a major source of heavy metal contamination. Ion imprinted polymers (IIP) are similar to 
MIP and can be created to treat heavy metal contamination. Use of NIP for general water and wastewater applications may also result in the removal of heavy metals in addition to emerging contaminants. An investigation into the ability of NIP to remove heavy metals is required.

Because NIP are nonspecific, and remove contaminants via adsorption, they are most similar to powder activated carbon. Activated carbon can experience large reductions in adsorption capacity due to competitive adsorption of natural organic matter present in natural waters or wastewater. Reductions in adsorption capacity for activated carbon in natural waters typically occur due to pore blocking, where large molecules block the entrance into the internal pore structure of the activated carbon, or direct competition between natural organic matter and adsorbate molecules. Research is needed to identify the extent of competition for NIP in natural waters as well as the governing mechanisms of the competition. A full understanding of any competition may allow for better design of NIP and improved performance.

The polymeric particles, particularly NIP, are inexpensive to manufacture and can be produced in large quantities. Furthermore, the particles can be regenerated and reused repeatedly. The particles would need to be removed after their application and this can be achieved through physical, chemical or magnetic separation techniques. More research is required to determine how best to incorporate MIP and NIP into treatment plants. The opportunities and research gaps identified above determined the scope of the thesis and were chosen for further investigation in this thesis. 


\subsection{REFERENCES}

Adams, C., and S. Randtke. 1992 Ozonation Byproducts of Atrazine in Synthetic and Natural Waters. Environmental Science \& Technology, 26 (11), 2218-2227.

Alexander, C. 2006 Molecular Imprinting Science and Technology: a Survey of the Literature for the Years up to and Including 2003. Journal of Molecular Recognition, 19 (2), 106-80.

Andersson, H. S., a C. Koch-Schmidt, S. Ohlson, and K. Mosbach. 1996 Study of the Nature of Recognition in Molecularly Imprinted Polymers. Journal of Molecular Recognition, 9 (5-6), 675-82.

Arora, M., B. Kiran, S. Rani, A. Rani, B. Kaur, and N. Mittal. 2008 Heavy Metal Accumulation in Vegetables Irrigated with Water from Different Sources. Food Chemistry, 111 (4), 811-815.

Ashraf, S., A. Cluley, C. Mercado, and A. Mueller. 2011 Imprinted Polymers for the Removal of Heavy Metal Ions from Water. Water Science and Technology, 64 (6), 1325-32.

Bauman, R. 2004 Specific Defense: The Immune Response. Microbiology, (pp. 463-486). Pearson Benjamin Cummings, San Francisco.

Benjamin, M., 2002. Water Chemistry. McGraw Hill, Boston.

Bjelopavlic, M., G. Newcombe, and R. Hayes. 1999 Adsorption of NOM onto Activated Carbon: Effect of Surface Charge, Ionic Strength, and Pore Volume Distribution. Journal of Colloid and Interface Science, 210 (2), 271-280. 
Bögi, C., J. Schwaiger, H. Ferling, U. Mallow, C. Steineck, F. Sinowatz, W. Kalbfus, RD Negele, I. Lutz, W. Kloas. 2003 Endocrine Effects of Environmental Pollution on Xenopus laevis and Rana temporaria. Environmental Research, 93 (2), 195-201.

Braeken, L., and B. Van der Bruggen. 2009 Feasibility of Nanofiltration for the Removal of Endocrine Disrupting Compounds. Desalination, 240 (1-3), 127-131.

Branger, C., W. Meouche, and A. Margaillan. 2013 Recent Advances on Ion-Imprinted Polymers. Reactive and Functional Polymers, 73 (6), 859-875.

Bravo, J. C., R. M. Garcinuño, P. Fernández, and J. S. Durand. 2007 A New Molecularly Imprinted Polymer for the On-Column Solid-Phase Extraction of Diethylstilbestrol from Aqueous Samples. Analytical and Bioanalytical Chemistry, 388 (5-6), 1039-1045.

Chelating Agents in Medicine. 1971 British Medical Journal, (May), 270-272.

Chiou, C. T. 2002 Partition and Adsorption of Organic Contaminants in Environmental Systems. Wiley-Interscience, Hoboken, N.J.

Chowdhury, B. 1987 Biological and Health Implications of Toxic Heavy Metal and Essential Trace Element Interactions. Progress in Food \& Nutrition Science, 11 (1), 55-113.

Clara, M., N. Kreuzinger, B. Strenn, O. Gans, and H. Kroiss. 2005 The Solids Retention Time-a Suitable Design Parameter to Evaluate the Capacity of Wastewater Treatment Plants to Remove Micropollutants. Water Research, 39 (1), 97-106.

Deng, S., D. Shuai, Q. Yu, J. Huang, and G. Yu. 2009 Selective Sorption of Perfluorooctane Sulfonate on Molecularly Imprinted Polymer Adsorbents. Frontiers of Environmental Science \& Engineering in China, 3 (2), 171-177. 
Ding, L., V. L. Snoeyink, B. J. Mariñas, Z. Yue, and J. Economy. 2008 Effects of Powdered Activated Carbon Pore Size Distribution on the Competitive Adsorption of Aqueous Atrazine and Natural Organic Matter. Environmental Science \& Technology, 42 (4), 1227-31.

Ebie, K. 1995 Effect of Pore Size Distribution of Activated Carbon on the Adsorption of Humic Substances and Trace Organic Compounds. Water Supply, 13 (3-4), 6570.

Ebie, K., F. Li, Y. Azuma, A. Yuasa, and T. Hagishita. 2001 Pore Distribution Effect of Activated Carbon in Adsorbing Organic Micropollutants from Natural Water. Water Research, 35 (1), 167-79.

Ensing, K., and T. De Boer. 1999 Tailor-Made Materials for Tailor-Made Applications: Application of Molecular Imprints in Chemical Analysis. TrAC - Trends in Analytical Chemistry, 18 (3), 138-145.

Fang, C., and S. Li. 2007 Thermodynamic and Kinetic Considerations on the Specific Adsorption and Molecular Recognition by Molecularly Imprinted Polymer. Journal of Inorganic and Organometallic Polymers and Materials, 17 (4), 623629.

Fernández-Alvarez, P., M. Le Noir, and B. Guieysse. 2009 Removal and Destruction of Endocrine Disrupting Contaminants by Adsorption with Molecularly Imprinted Polymers Followed by Simultaneous Extraction and Phototreatment. Journal of Hazardous Materials, 163 (2-3), 1107-12. 
Fuerhacker, M., A. Dürauer, and A. Jungbauer. 2001 Adsorption Isotherms of 17 $\beta$ Estradiol on Granular Activated Carbon (GAC). Chemosphere, 44 (7), 15731579.

Gillesby, B. E., and T. R. Zacharewski. 1998 Exoestrogens: Mechanisms of Action and Strategies for Identification and Assessment. Environmental Toxicology and Chemistry, 17 (1), 3-14.

Guo, W., W. Hu, J. Pan, H. Zhou, W. Guan, X. Wang, J. Dai, et al. 2011 Selective Adsorption and Separation of BPA from Aqueous Solution Using Novel Molecularly Imprinted Polymers Based on Kaolinite/Fe3O4 Composites. Chemical Engineering Journal, 171 (2), 603-611.

Guo, Y. C., and S. W. Krasner. 2009 Occurrence of Primidone, Carbamazepine, Caffeine, and Precursors for N -Nitrosodimethylamine in Drinking Water Sources Impacted by Wastewater. JAWRA Journal of the American Water Resources Association, $45(1), 58-67$.

Hajizadeh, S., H. Kirsebom, I. Y. Galaev, and B. Mattiasson. 2010 Evaluation of Selective Composite Cryogel for Bromate Removal from Drinking Water. Journal of Separation Science, 33 (12), 1752-9.

Helfman, G. S. 2007 Fish Conservation: a Guide to Understanding and Restoring Global Aquatic Biodiversity and Fishery Resources, Island Press, Washington D.C.

Houston, M. C. 2011 Role of Mercury Toxicity in Hypertension, Cardiovascular Disease, and Stroke. Journal of Clinical Hypertension, 13 (8), 621-627.

Jarup, L. 2003 Hazards of Heavy Metal Contamination. British Medical Bulletin, 68 (1), 167-182. 
Karge, H., and J. Weitkamp. 2008 Adsorption and Diffusion. Springer-Verlag, Berlin.

Konig, B. 1995 Molecular Recognition. The Principle and Recent Chemical Examples. Journal für praktische Chemie, 337 339-346.

Krupadam, R. J., M. S. Khan, and S. R. Wate. 2010 Removal of Probable Human Carcinogenic Polycyclic Aromatic Hydrocarbons from Contaminated Water Using Molecularly Imprinted Polymer. Water Research, 44 (3), 681-8.

Lai, E. P. C., M. Hassanzadeh-Khayyat, and G. Do. 2011 Degradation Kinetics of Pharmaceuticals in Molecularly Imprinted Polymer Particles by Acid and Hydrogen Peroxide Treatments. Clinical Biochemistry, 44 (13), S8.

Le Noir, M., A.-S. Lepeuple, B. Guieysse, and B. Mattiasson. 2007a Selective Removal of 17Beta-Estradiol at Trace Concentration Using a Molecularly Imprinted Polymer. Water Research, 41 (12), 2825-31.

Le Noir, M., F. Plieva, T. Hey, B. Guieysse, and B. Mattiasson. 2007b Macroporous Molecularly Imprinted Polymer/Cryogel Composite Systems for the Removal of Endocrine Disrupting Trace Contaminants. Journal of Chromatography. A, 1154 $(1-2), 158-64$.

Le Noir, M., F. M. Plieva, and B. Mattiasson. 2009 Removal of Endocrine-Disrupting Compounds from Water Using Macroporous Molecularly Imprinted Cryogels in a Moving-Bed Reactor. Journal of Separation Science, 32 (9), 1471-9.

Li, Q., V. L. Snoeyink, B. J. Mariñas, and C. Campos. 2003 Pore Blockage Effect of NOM on Atrazine Adsorption Kinetics of PAC: The Roles of PAC Pore Size Distribution and NOM Molecular Weight. Water Research, 37 (20), 4863-4872. 
Li, Y., X. Li, Y. Li, J. Qi, J. Bian, and Y. Yuan. 2009 Selective Removal of 2,4Dichlorophenol from Contaminated Water Using Non-Covalent Imprinted Microspheres. Environmental Pollution, 157 (6), 1879-85.

Liao, T., X. Tu, and S. Li. 2007 Selective Adsorption and Recognition by Molecularly Imprinted Polymer: A Study on Molecular Self-Assembly and its Effect on Selectivity. Polymer-Plastics Technology and Engineering, 46 (6), 613-619.

Lin, Y., Y. Shi, M. Jiang, Y. Jin, Y. Peng, B. Lu, and K. Dai. 2008 Removal of Phenolic Estrogen Pollutants from Different Sources of Water using Molecularly Imprinted Polymeric Microspheres. Environmental Pollution, 153 (2), 483-91.

Luo, X., Y. Zhan, Y. Huang, L. Yang, X. Tu, and S. Luo. 2011 Removal of Water-Soluble Acid Dyes from Water Environment Using a Novel Magnetic Molecularly Imprinted Polymer. Journal of Hazardous Materials, 187 (1-3), 274-82.

Mapanda, F., E. N. Mangwayana, J. Nyamangara, and K. E. Giller. 2005 The Effect of Long-Term Irrigation Using Wastewater on Heavy Metal Contents of Soils Under Vegetables in Harare, Zimbabwe. Agriculture, Ecosystems and Environment, 107 (2-3), 151-165.

Masqué, N. 2001 Molecularly Imprinted Polymers: New Tailor-Made Materials for Selective Solid-Phase Extraction. TrAC Trends in Analytical Chemistry, 20 (9), $477-486$.

Matsui, Y., Y. Fukuda, T. Inoue, and T. Matsushita. 2003 Effect of Natural Organic Matter on Powdered Activated Carbon Adsorption of Trace Contaminants: Characteristics and Mechanism of Competitive Adsorption. Water Research, 37 (18), 4413-4424. 
Meng, Z., W. Chen, and A. Mulchandani. 2005 Removal of Estrogenic Pollutants from Contaminated Water Using Molecularly Imprinted Polymers. Environmental Science \& Technology, 39 (22), 8958-62.

Meng, Z., Q. Zhang, M. Xue, D. Wang, and A. Wang. 2012 Removal of 2,4,6Trinitrotoluene from "Pink Water" Using Molecularly-Imprinted Absorbent. Propellants, Explosives, Pyrotechnics, 37 (1), 100-106.

Murray, Audrey. Evaluation of Molecularly Imprinted and Non-imprinted Nanoparticles for Removal of Endocrine Disrupting Compounds from Surface Water and Wastewater. Thesis. Carleton University, 2010. Ottawa: Carleton U, 2010. Print.

Murray, A., B. Ormeci, and E. P. C. Lai. 2011 Removal of 17 Beta-Estradiol (E2) and its Chlorination by-Products from Water and Wastewater Using Non-Imprinted Polymer (NIP) Particles. Water Science and Technology, 64 (6), 1291-1297.

Nagajyoti, P. C., K. D. Lee, and T. V. M. Sreekanth. 2010 Heavy Metals, Occurrence and Toxicity for Plants: A Review. Environmental Chemistry Letters, 8 (3), 199-216. Newcombe, G., and M. Drikas. 1997 Adsorption of NOM onto Activated Carbon: Electrostatic and Non-Electrostatic Effects. Carbon, 35 (9), 1239-1250.

Newcombe, G., J. Morrison, C. Hepplewhite, and D. R. U. Knappe. 2002 Simultaneous Adsorption of MIB and NOM onto Activated Carbon II: Competitive Effects. Carbon, 40 2147-2156.

Pelekani, C., and V. . Snoeyink. 1999 Competitive Adsorption in Natural Water: Role of Activated Carbon Pore Size. Water Research, 33 (5), 1209-1219. 
Pelekani, C., and V. L. Snoeyink. 2000 Competitive Adsorption Between Atrazine and Methylene Blue on Activated Carbon: The Importance of Pore Size Distribution. Carbon, 38 (10), 1423-1436.

Pelekani, C., and V. L. Snoeyink. 2001 Kinetic and Equilibrium Study of Competitive Adsorption Between Atrazine and Congo Red Dye on Activated Carbon: The Importance of Pore Size Distribution. Carbon, 39 (1), 25-37.

Peng-Ju, W., Y. Jun, S. Qing-De, G. Yun, Z. Xiao-Lan, and C. Ji-Bao. 2007 Rapid Removal of Template from Molecularly Imprinted Polymers by Accelerated Solvent Extraction. Chinese Journal of Analytical Chemistry, 35 (4), 484-488.

Pichon, V. 1997 Environmental Sample Treatment: Evolution and Perspectives. Analysis, 25 (4), 101-106.

Pichon, V., and F. Chapuis-Hugon. 2008 Role of Molecularly Imprinted Polymers for Selective Determination of Environmental Pollutants--a Review. Analytica Chimica Acta, 622 (1-2), 48-61.

Quinlivan, P. a., L. Li, and D. R. U. Knappe. 2005 Effects of Activated Carbon Characteristics on the Simultaneous Adsorption of Aqueous Organic Micropollutants and Natural Organic Matter. Water Research, 39 (8), 1663-1673.

Randhawa, M., I. Gartner, and C. Becker. 2007 Imprinted Polymers for Water Purification. Journal of Applied Polymer Science, 106 (5), 3321-3326.

Rao, T. P., R. Kala, and S. Daniel. 2006 Metal Ion-Imprinted Polymers-Novel Materials for Selective Recognition of Inorganics. Analytica Chimica Acta, 578 (2), 105116. 
Redding, A. M., and F. S. Cannon. 2014 The Role of Mesopores in MTBE Removal with Granular Activated Carbon. Water Research, 56 214-24.

Rossner, A., S. a Snyder, and D. R. U. Knappe. 2009 Removal of Emerging Contaminants of Concern by Alternative Adsorbents. Water Research, 43 (15), 3787-96.

Ryan, J., H. Pahren, and J. Lucas. 1982 Controlling Cadmium in the Human Food Chain: a Review and Rationale Based on Health Effects. Environmental Research, 28 (2), 251-302.

Segner, H., K. Caroll, M. Fenske, C. R. Janssen, G. Maack, D. Pascoe, and C. Sch. 2003 Identification of Endocrine-Disrupting Effects in Aquatic Vertebrates and Invertebrates : Report from the European IDEA project 54 302-314.

Sellergren, B. 1999 Polymer-and Template-Related Factors Influencing the Efficiency in Molecularly Imprinted Solid-Phase Extractions. TrAC Trends in Analytical Chemistry, 18 (3), 164-174.

Senesi, G. S., G. Baldassarre, N. Senesi, and B. Radina. 1999 Trace Element Inputs into Soils by Anthropogenic Activities and Implications for Human Health. Chemosphere, 39 (2), 343-377.

Sharma, R. K., and M. Agrawal. 2005 Biological Effects of Heavy Metals: An Overview. Journal of Environmental Biology, 26 (2 SUPPL.), 301-313.

Shen, X., L. Zhu, N. Wang, L. Ye, and H. Tang. 2012 Molecular Imprinting for Removing Highly Toxic Organic Pollutants. Chemical Communications (Cambridge, England), 48 (6), 788-98.

Singh, A., R. K. Sharma, M. Agrawal, and F. M. Marshall. 2010 Health Risk Assessment of Heavy Metals via Dietary Intake of Foodstuffs from the Wastewater Irrigated 
Site of a Dry Tropical Area of India. Food and Chemical Toxicology, 48 (2), $611-$ 619.

Singh, R., N. Gautam, and R. Gupta. 2011 Heavy Metals and Living Systems: An Overview. Indian Journal of Pharmacology, 43 (3), 246-253.

Snyder, S. a., S. Adham, A. M. Redding, F. S. Cannon, J. DeCarolis, J. Oppenheimer, E. C. Wert, et al. 2007 Role of Membranes and Activated Carbon in the Removal of Endocrine Disruptors and Pharmaceuticals. Desalination, 202 (1-3), 156-181.

Spivak, D. A. 2005 Selectivity in Molecularly Imprinted Matrices. In M. YAN \& O. RAMSTRÖM, eds., Molecularly Imprinted Materials - Science and Technology, (pp. 395-418). MARCEL DEKKER.

Toze, S. 2006 Reuse of Effluent Water - Benefits and Risks. Agricultural Water Management, 80 (1-3 SPEC. ISS.), 147-159.

Vieno, N., T. Tuhkanen, and L. Kronberg. 2006 Removal of Pharmaceuticals in Drinking Water Treatment: Effect of Chemical Coagulation. Environmental Technology, 27 (2), 183-192.

Wang, X., T. Sato, B. Xing, and S. Tao. 2005 Health Risks of Heavy Metals to the General Public in Tianjin, China Via Consumption of Vegetables and Fish. Science of the Total Environment, 350 (1-3), 28-37.

Wulff, G. 1995 Molecular Imprinting in Cross-Linked Materials with the Aid of Molecular. Angewandte Chemie International Edition in English, 34 (17), 1812-1832.

Xia, X., E. P. C. Lai, and B. Örmeci. 2013 Duo-Molecularly Imprinted Polymer-Coated Magnetic Particles for Class-Selective Removal of Endocrine-Disrupting 
Compounds from Aqueous Environment. Environmental Science and Pollution Research, 20 (5), 3331-3339.

Xie, Y., H. Li, L. Wang, Q. Liu, Y. Shi, H. Zheng, M. Zhang, Y. Wu, B. Lu. 2011 Molecularly Imprinted Polymer Microspheres Enhanced Biodegradation of Bisphenol A by Acclimated Activated Sludge. Water Research, 45 (3), 1189-98.

Yan, H., and K. H. Row. 2006 Characteristic and Synthetic Approach of Molecularly Imprinted Polymer. International Journal of Molecular Sciences, 7 (5), 155-178.

Yang, M., M. S. Park, and H. S. Lee. 2006 Endocrine Disrupting Chemicals: Human Exposure and Health Risks. Journal of Environmental Science and Health. Part C, Environmental Carcinogenesis \& Ecotoxicology Reviews, 24 (2), 183-224.

Yu, Q., S. Deng, and G. Yu. 2008 Selective Removal of Perfluorooctane Sulfonate from Aqueous Solution Using Chitosan-Based Molecularly Imprinted Polymer Adsorbents. Water Research, 42 (12), 3089-97.

Zhang, Q., S. Deng, G. Yu, and J. Huang. 2011 Removal of Perfluorooctane Sulfonate from Aqueous Solution by Crosslinked Chitosan Beads: Sorption Kinetics and Uptake Mechanism. Bioresource Technology, 102 (3), 2265-71.

Zhang, Z., and J. Hu. 2009 Effect of Environmental Factors on Estrogenic Compounds Adsorption by MIP. Water, Air, \& Soil Pollution, 210 (1-4), 255-264.

Zhongbo, Z., and J. Hu. 2008 Selective Removal of Estrogenic Compounds by Molecular Imprinted Polymer (MIP). Water Research, 42 (15), 4101-8. 


\section{CHAPTER 4: MATERIALS AND METHODS}

\subsection{NIP SYNTHESIS}

The NIP solution was mixed under a fume hood. Functional monomer methacrylic acid (MAA) (Sigma-Aldrich; Oakville, Canada) and cross-linker ethylene glycol dimethacrylate (EGDMA) (Sigma-Aldrich; Oakville, Canada) were dissolved in a porogen with a molar ratio of 8mmol:6.7mmol (Wei et al., 2006). $5 \mathrm{~mL}$ of MAA and EGDMA were filtered through columns containing $2.5 \mathrm{~g}$ of activated alumina each to remove the inhibitors added by the manufacturer. A porogen containing $40 \mathrm{~mL}$ of $1: 3(\mathrm{v}: \mathrm{v})$ acetone (Fisher Scientific; Ottawa, Canada) and acetonitrile (Fisher Scientific: Ottawa, Canada) was prepared while the MAA and EGDMA were being filtered. Then, $686 \mu \mathrm{L}$ of the MAA and $1328 \mu \mathrm{L}$ of EGDMA were added to the porogen. $2 \%$ by weight of an initiator, 2 isobutyronitrile (AIBN), which was kept in the freezer prior to use, was then added the NIP solution. The total weight of the NIP solution was calculated using a density of $0.791 \mathrm{~g} / \mathrm{cm}^{3}$ for acetone and $0.786 \mathrm{~g} / \mathrm{cm}^{3}$ for acetonitrile as well as the masses of MAA and EGDMA added (the densities of MAA and EGDMA, as reported by Sigma-Aldrich, are approximately $1 \mathrm{~g} / \mathrm{mL}$ ) as shown below for a $40 \mathrm{~mL}$ total solution. Note: $1 \mathrm{~cm}^{3}=1 \mathrm{~mL}$.

Total mass $=\left(0.786 \frac{\mathrm{g}}{\mathrm{cm}^{3}}\right)\left(30 \mathrm{~cm}^{3}\right)+\left(0.791 \frac{\mathrm{g}}{\mathrm{cm}^{3}}\right)\left(10 \mathrm{~cm}^{3}\right)+0.686 \mathrm{~g}+1.328 \mathrm{~g}$

Total mass $=33.5 \mathrm{~g}$

$2 \%$ of the total mass of the solution, or $0.67 \mathrm{~g}$, of the initiator was added to the NIP solution. The mixture was mixed with a vortex mixer (Fisher Scientific Vortex Mixer, USA), 
deoxygenated with nitrogen for five minutes, and then placed in a $60^{\circ} \mathrm{C}$ hot water bath for 24h (Isotemp 220, Fisher, USA). The resulting polymer particles were dewatered using a centrifuge (Thermo Scientific Sorval Legend $\mathrm{RT}^{+}$, Fisher Scientific) at 10,000 RPM, air dried at room temperature, and ground manually.

The above method describes the procedure followed to make $40 \mathrm{~mL}$ of NIP. At various times throughout this project, smaller or larger batches of NIP were created. In all cases, care was taken to ensure that the airspace at the top of the vials in which the NIP were placed prior to being deoxygenated was minimized (vials of an appropriate volume were used $-50 \mathrm{~mL}$ for $40 \mathrm{~mL}$ of solution and $100 \mathrm{~mL}$ for $80 \mathrm{~mL}$ of solution). It was important to leave some airspace so that the vials did not overflow when the nitrogen gas was bubbled into the solution. For batches larger than $80 \mathrm{~mL}$, the NIP solution was separated into 100 $\mathrm{mL}$ vials prior to deoxygenating to limit the surface area at the top of the solution.

\subsection{GLASSWARE CLEANING}

\subsubsection{EDCs and pharmaceuticals}

Glassware contaminated with EDCs or pharmaceuticals was washed with laboratory soap and water, rinsed three times with acetonitrile (Fisher Scientific; Ottawa, Canada), three times with methanol (Fisher Scientific; Ottawa, Canada), and once with deionized water prior to being re-used. Alternatively, the glassware could have been baked at $550{ }^{\circ} \mathrm{C}$. 


\subsubsection{Natural organic matter (NOM) and heavy metals}

Glassware contaminated with NOM (from humic acid, natural waters, or wastewater) or heavy metals was soaked in an acid bath containing $0.2 \mathrm{M}$ of hydrochloric acid (Anachemia; Montreal, Canada) for a minimum of 24 hours. It was then rinsed and airdried.

\subsection{USE OF NON-IMPRINTED POLYMERS FOR REMOVAL OF ENDOCRINE DISRUPTINGCOMPOUNDS AND PHARMACEUTICALS FROM WATER AND WASTEWATER}

\subsection{Micropollutants}

Endocrine disrupting compounds (EDCs) and pharmaceuticals: $17-\beta$ estradiol (E2), 17- $\alpha$ ethinylestradiol (EE2), estrone(E1), diethylstilbestrol (DES), bisphenol A (BPA), acetaminophen, caffeine, carbamazepine, cloxacillin, diphenhydramine, enrofloxacin, lincomycin, oxacillin, sulfadiazine, sulfamethizole, sulfanilamide, and sulfathiazole were all purchased from Sigma-Aldrich (St Louis, Missouri).

$1 \mathrm{mg} / \mathrm{mL}$ stock solutions were prepared in methanol and stored in the freezer prior to use. Serial dilutions were prepared in methanol, diluting into the sample water in the last step. For example, a 2 ppm standard solution of E2 in deionized water was prepared by first preparing a $100 \mathrm{ppm}$ E2 solution in methanol by adding $500 \mu \mathrm{L}$ of the $1 \mathrm{mg} / \mathrm{mL}$ stock solution to $4500 \mu \mathrm{L}$ of methanol and mixing with a vortex mixer (Fisher Scientific Vortex Mixer, USA). $400 \mu \mathrm{L}$ of the $100 \mathrm{ppm}$ E2 standard was then added to $19.6 \mathrm{~mL}$ of deionized water to obtain $20 \mathrm{~mL}$ of the $2 \mathrm{ppm}$ E2 standard solution. 


\subsubsection{Analytical measurements}

\subsubsection{Single EDC analysis}

Analysis was conducted using high-performance liquid chromatography (HPLC) with a Phenomenex $50 \times 2.00 \mathrm{~mm}$ column and a mobile phase with 55:45 methanol:deionized water. The ratio of methanol to deionized water was adjusted to provide optimum peak sharpness and retention time. A Shimadzu LC 20 AD HPLC with a Sil 20A auto sampler (Shimadzu; Kyoto, Japan) was used for all analysis. Fluorescence detection (FD) with a RF-10AXL detector (Shimadzu; Kyoto, Japan) was used for E2, EE2 and BPA, and photo diode array (PDA) detection with a SPD-M20A detector (Shimadzu; Kyoto, Japan) was used for DES. The limits of detection were $0.1 \mathrm{ppb}$ for E2, EE2 and BPA, and $0.25 \mathrm{ppm}$ for DES.

An 8 minute HPLC program was run with a flowrate of $1 \mathrm{~mL} /$ minute. FD with E2, EE2, and BPA was conducted using an excitation wavelength of $280 \mathrm{~nm}$ and an emission wavelength of $310 \mathrm{~nm}$ as suggested by Yoon et al (2003) (Yoon et al., 2003). Some optimization of the fluorescence wavelength was conducted, but 280 and $310 \mathrm{~nm}$ were

found to give the best response. The PDA scanned between 190-800nm for analysis of DES.

\subsubsection{Treatment of water with a mixture of EDCs and removal kinetics}

Analysis was conducted using high performance liquid chromatography tandem mass spectrometry (LC/MS/MS) in MRM mode. A Shimadzu HPLC (LC 20 AD) was used in conjunction with an AB Sciex API 2000 triple quadrupole tandem mass spectrometer 
(Framingham, Massachusetts). The HPLC column used was a Phenomenex Kinetex $2.6 \mu$ PFP column. Electrospray ionization was applied in negative mode for the mass spectrometer. Both positive mode and negative mode were tested and negative mode provided the best results.

Optimization of the LC/MS/MS parameters was conducted prior to analysis by first using the automatic optimization program associated with the software (Analyst; AB Sciex; Framingham, Massachusetts) to obtain a range of parameters, and then using manual tuning to fine-tune the optimization. Compound specific parameters were adjusted for each EDC in turn, using a $0.5 \mathrm{ppm}$ standard solution prepared in the mobile phase and injected via direct infusion. Compound specific parameters included: Q1 (compound) mass, Q3 (product ion) mass, declustering potential (DP), focusing potential (FP), entrance potential (EP), collision energy (CE), and collision cell exit potential (CXP). A trial-and-error process was used to maximize the signal for each compound specific parameter in turn for each EDC.

Estrone (E1) was found to have the weakest signal following optimization, and was identified as the priority for optimization of the source specific parameters (curtain gas, collision gas, ion spray voltage, temperature, and ion source gases 1 and 2). A standard solution containing $0.5 \mathrm{ppm}$ each of each EDC was injected via direct infusion, but the E1 response was maximized. Signals from the other EDCs were observed to ensure that they did not fall below that for E1. Calibration curves were created for each of the 5 EDCs and used to calculate the concentrations after treatment. 


\subsubsection{Removal of pharmaceuticals}

Analysis was conducted by Axys Analytical Services (Sidney, BC) using LC/MS/MS (Axys Method MLA-075 Rev 5). The Axys Analytical Services method is certified by the Canadian Association for Laboratory Accreditation and corresponds to USEPA Method 1694(Certificate of accreditation - Canadian Association for Laboratory Accreditation, 2010). The Axys Analytical method uses formic acid to adjust the $\mathrm{pH}$ of the sample to 2 prior to solid phase extraction followed by LC/MS/MS analysis (Englert, 2007). Refer to Appendix A for the reporting limits of the analysis.

The USEPA Method 1694 is for analysis of pharmaceuticals and personal care products in water, soil, sediments, and biosolids using HPLC/MS/MS. This method is divided into 4 lists. All of the pharmaceuticals measured herein were from List 1. Aqueous samples containing List 1 analytes are filtered if they contain any visible particles and the $\mathrm{pH}$ is adjusted to 2. Stable isotope analogues are injected and the solution is stabilized with tetrasodium ethylenediamine-tetraacetatedihydrate. Solid phase extraction is performed using hydrophilic lipophillic balance cartridges, and the analyte is transferred to methanol. Radiolabelled injection standards are added and the final volume is adjusted to $4 \mathrm{~mL}$. The sample is then analyzed using LC/MS/MS (Englert, 2007).

\subsubsection{Identification of important parameters}

Preliminary experiments were performed using E2 as a model compound to investigate the effect of NIP mass, binding time, E2 concentration, and NIP batch on the adsorption of E2 onto NIP. This was necessary to design the future experiments in a way that focused on the 
most important variables. NIP concentrations of 5, 10, 15, or $20 \mathrm{mg} / \mathrm{L}$; binding times of 5, 7.5, or 10 minutes; E2 concentrations of 2, 6, 10, or 14 ppm; and two separate NIP batches were tested. PH was not monitored.

Conditions tested were:

Table 4-1: Identification of important parameters

\begin{tabular}{|c|c|c|c|}
\hline $\begin{array}{c}\text { NIP Concentration } \\
(\mathrm{x} 4)(\mathrm{mg} / \mathrm{L})\end{array}$ & $\begin{array}{c}\text { Binding Times }(\mathrm{x} 3) \\
(\text { minutes })\end{array}$ & $\begin{array}{c}\text { E2 Concentration } \\
(\mathrm{x} 4)(\mathrm{ppm})\end{array}$ & Batch $(\mathrm{x} 2)$ \\
\hline 5 & 5 & 2 & 1 \\
\hline 10 & 7.5 & 6 & 2 \\
\hline 15 & 10 & 10 & \\
\hline 20 & & 14 & \\
\hline
\end{tabular}

TOTAL Samples $=4 \times 3 \times 4 \times 2=96$ samples

Due to the large number of conditions tested, replicates were not performed for each combination. Instead, 3 replicates were performed for the following combinations: $8.5 \mathrm{ppm}$ E2 with $5,12.5$, or $20 \mathrm{mg} / \mathrm{L}$ of NIP, and a 7.5 minute contact time, for NIP batch 1 or 2 . This resulted in a total of 6 sets of 3 replicates. These replicates were used to estimate a pooled variance for all of the results.

The tests were conducted by weighing the appropriate mass of NIP into $1.5 \mathrm{~mL}$ plastic centrifuge tubes and adding $1 \mathrm{~mL}$ of deionized water spiked with the selected $\mathrm{E} 2$ concentration. The samples were sonicated (Vibracell Sonics; Sonics and Materials Inc.; 
Newtown, Connecticut) for the required contact time. Following sonification, the tubes were centrifuged (Sorvall Micro 21; Thermo Scientific; Waltham, Massachusetts) for 5 minutes at $10,000 \mathrm{rpm}$ to remove the NIP. The centrate was then analyzed with HPLC (Shimadzu LC 20 AD; Kyoto, Japan) using fluorescence detection (FD) (Shimadzu RF10AXL; Kyoto, Japan).

\subsubsection{Treatment of water with a single EDC}

NIP were tested for their ability to remove EE2, DES, and BPA from deionized water through a series of batch tests each with a single solute. For each sample, $5 \mathrm{mg}$ of NIP was weighed into a $1.5 \mathrm{~mL}$ centrifuge tube, and $1 \mathrm{~mL}$ of deionized water spiked with the relevant concentration of EE2, DES, or BPA was added. NIP from batch 1 were used so that the results could be compared with those obtained for E2. There was significant batch to batch variation in adsorption of E2, so it was important to use NIP from the same batch. Multiple batches of NIP were prepared and mixed together for use in all remaining experiments. The tubes were sonicated for 5 minutes to disperse the NIP before centrifugation at 10,000 rpm for 5 minutes. The centrate was analyzed using HPLC as previously described. Three experimental replicates each consisting of two analytical replicates were performed. For EE2 and BPA, concentrations were varied between 2-14 $\mathrm{ppb}$, and for DES, concentrations of 5-20 ppm were tested. PH was not monitored.

\subsubsection{Treatment of water with a mixture of EDCs}

NIP were also tested for treatment of a mixture of EDCs consisting of $0.5 \mathrm{ppm}$ each of E2, EE2, E1, BPA, and DES over a 24 hour contact time. NIP concentrations of $0.1,1,10,100$, 
and $1000 \mathrm{mg} / \mathrm{L}$ were tested to establish the range over which NIP were effective. $200 \mathrm{~mL}$ amber glass bottles were filled with $50 \mathrm{~mL}$ each of deionized water. Next, a concentrated NIP solution was created and sonicated for 15 minutes before it was pipetted into the bottles to achieve the desired final concentration. Then, a mixture of E2, EE2, E1, BPA, and DES, each with a concentration of $25 \mathrm{ppm}$, was created in methanol and $1 \mathrm{~mL}$ was pipetted into each of the bottles, to obtain a final concentration of $0.5 \mathrm{ppm}$ for each EDC. The bottles were placed on a shaker table (Excella E1 Platform Shaker; New Brunswick Scientific Inc.;

Enfield, Connecticut) for 24 hours. The following day, the samples were centrifuged in plastic centrifuge tubes at 10,000 rpm (670,800 g) for 1 hour (Sorval Legend RT+; Thermo Scientific/Fisher Scientific; Waltham, Massachusetts), and the centrate was analyzed with LC/MS/MS as previously described. Three experimental replicates, each consisting of 6 injection replicates, were conducted for each sample. PH was not monitored.

\subsubsection{Establishing the kinetics of removal}

The kinetics of removal were analyzed using a 0.5 ppm mixture of E2, EE2, E1, BPA, and DES following the same procedure as that outlined above for treatment of water with a mixture of EDCs. NIP concentrations of 50 and $500 \mathrm{mg} / \mathrm{L}$ were tested for contact times of 10 minutes, 30 minutes, 1 hour, 2 hours, 5 hours, 1 day, and 4 days. PH was not monitored.

\subsubsection{Removal of pharmaceuticals}

Tests were conducted with 12 pharmaceuticals: acetaminophen, caffeine, carbamazepine, cloxacillin, diphenhydramine, enrofloxacin, lincomycin, oxacillin, sulfadiazine, sulfamethizole, sulfanilamide, and sulfathiazole in deionized water and wastewater. 
Secondary wastewater effluent samples were collected from the Robert O. Pickard Environmental Centre in Ottawa, Ontario. Refer to Appendix B for a summary of the wastewater characteristics. $200 \mathrm{ng} / \mathrm{L}$ of acetaminophen, $50 \mathrm{ng} / \mathrm{L}$ of sulfathiazole, and 100 $\mathrm{ng} / \mathrm{L}$ of the remaining pharmaceuticals were spiked into $2.5 \mathrm{~L}$ each of deionized water and wastewater. A $100 \mathrm{~g} / \mathrm{L}$ solution of NIP was created and sonicated to disperse the NIP. $1 \mathrm{~L}$ of each of the spiked solutions was treated by adding $10 \mathrm{~mL}$ of the concentrated NIP solution to give a final concentration of $1 \mathrm{~g} \mathrm{NIP/L}$. The samples were mixed for 24 hours on a shaker table before being centrifuged at 10,000 rpm for 1 hour to remove the NIP. The centrate was poured into sample bottles, which were packed in a cooler and shipped to Axys Analytical Services (Sidney, BC). PH was not monitored.

\subsection{USE OF NON-IMPRINTED POLYMERS FOR REMOVAL OF HEAVY METAL IONS FROM WATER AND WASTEWATER}

\subsubsection{Analytical measurements}

Heavy metal analysis was carried out at the ICP-MS facility at the University of Ottawa. Quantitative analysis of heavy metals was conducted with an Agilent 8800 triple quadrapole inductively coupled plasma mass spectrometer at an RF power of $1500 \mathrm{~kW}$, gas flow of $1.07 \mathrm{~L} /$ minute, and sample uptake rate of $0.2 \mathrm{~mL} /$ minute. A total of 8 lab blanks were run prior to sample analysis, and lab standards were analyzed between each 10 samples. 


\subsubsection{Experiments with environmental samples}

NIP were tested for their ability to remove lead, copper, nickel, and zinc from river water and wastewater samples. River water samples were collected from the Rideau River near Carleton University in Ottawa, Ontario. Secondary wastewater effluent samples were collected from the Robert O. Rickard Environmental Centre in Ottawa, Ontario. Heavy metal standard solutions at concentrations of $1000 \mathrm{mg} / \mathrm{L}$ in nitric acid were purchased from Sigma-Aldrich. Two serial dilutions, one with $250 \mathrm{ppm}$ of each metal and one with $10 \mathrm{ppm}$ of each metal were prepared in deionized water and neutralized with sodium hydroxide and hydrochloric acid as needed. The $\mathrm{pH}$ was measured using a Thermo Scientific Orion 5 Star $\mathrm{pH}$ meter.

River water and wastewater samples spiked with mixtures of $100 \mathrm{ppb}, 500 \mathrm{ppb}$, and 10 , $000 \mathrm{ppb}$ of each heavy metal were prepared by pipetting in serial dilutions in $15 \mathrm{~mL}$ centrifuge tubes. A $1000 \mathrm{mg} / \mathrm{L}$ NIP solution was created and sonicated (Vibracell Sonics, Sonics and Materials Inc., Newtown Connecticut) for 15 minutes to disperse the particles. $0.75 \mathrm{~mL}$ of the concentrated NIP solution was added to each tube to provide a final NIP concentration of $50 \mathrm{mg} / \mathrm{L}$. The samples were mixed on a shaker table (New Brunswick Scientific Excella E1 Platform Shaker, New Brunswick Scientific Inc.) for two weeks. They were then centrifuged (Thermo Scientific Sorval Legend RT+, Fisher Scientific) for 1 hour at a speed of $10,000 \mathrm{rpm}(670,800 \mathrm{~g})$. Following centrifugation, the centrate was carefully poured into new $15 \mathrm{~mL}$ centrifuge vials and the initial vials (containing the NIP particles) were discarded. Samples were acidified with $0.15 \mathrm{~mL}$ nitric acid (68-70\%, Anachemica, Montreal, Quebec) per $15 \mathrm{~mL}$ sample and stored at room temperature prior 
to analysis. Two experimental replicates were collected for each sample. PH was not monitored during the experiments. Control samples were also prepared with the sample water alone, and sample water with NIP particles added.

\subsubsection{Experiments with synthetic water}

NIP were also tested and compared with a powdered activated carbon (PAC $200 \mathrm{M}-2018$, Norit, Cabot Canada, Sarnia, Ontario) for removal of the heavy metal mixture in deionized water spiked with humic acid. Refer to Appendix $\mathrm{C}$ for information from the manufacturer about the activated carbon. Sample water was created with $20 \mathrm{mg} / \mathrm{L}$ of Suwannee River (II) humic acid obtained from the International Humic Substances Society. A detailed analysis of the humic acid, provided by the International Humic Substances Society, is available in Appendix D. The sample water was neutralized with sodium hydroxide (18\%) and hydrochloric acid (36.5-38\%). PH was not monitored after initial sample preparation.

Two sets of experiments were conducted:

(1) NIP particles and activated carbon were pre-loaded with humic acid before exposure to heavy metals. Pre-loading experiments were conducted to investigate the extent of pore-blocking.

(2) NIP particles and activated carbon were simultaneously mixed with both humic acid and heavy metals. Simultaneous loading experiments were conducted to investigate the relative extents of direct competition and pore blocking. A decrease in adsorption for simultaneous loading is indicative of competition, and a further 
decrease for pre-loading is indicative of pore-blocking (Pelekani and Snoeyink, 1999).

Control samples were also prepared with the sample water alone, sample water with activated carbon, and sample water with NIP particles added.

\subsubsection{Pre-loading experiments}

$1000 \mathrm{mg} / \mathrm{L}$ solutions of both NIP and activated carbon were produced as described above in Section 4.4.2 for the river water and wastewater samples. The particles were mixed with $15 \mathrm{~mL}$ of the humic acid sample at final adsorbent concentrations of $50 \mathrm{mg} / \mathrm{L}$ for the NIP and $10 \mathrm{mg} / \mathrm{L}$ for the activated carbon, for 24 hours on a shaker table. Following the mixing period, the samples were centrifuged for 1 hour at 10,000 rpm. The centrate was discarded, and $100 \mathrm{ppb}, 500 \mathrm{ppb}$, and 10,000 ppb mixtures of the four heavy metals (lead, copper, zinc, and nickel) at $\mathrm{pH} 7$ were added. The samples were mixed with a vortex mixer (Fisher Scientific Vortex Mixer, USA) to distribute the particles, followed by a 2 week mixing period on a shaker table. At the end of the two weeks, samples were centrifuged and treated as outlined above in Section 4.4.2.

\subsubsection{Simultaneous loading experiments}

For the simultaneous loading experiments, the $20 \mathrm{mg} / \mathrm{L}$ of humic acid, $50 \mathrm{mg} / \mathrm{L}$ NIP or 10 $\mathrm{mg} / \mathrm{L}$ activated carbon, and $100 \mathrm{ppb}, 500 \mathrm{ppb}$, or 10,000 ppb heavy metal mixtures were combined simultaneously and treated as outlined above in Section 4.4.2. 


\subsubsection{Adsorption isotherms and kinetics}

\subsubsection{Adsorption calculations and modelling}

\subsection{Percent removal by adsorption}

Percent removal was calculated based on the difference in concentrations between the control (containing no adsorbent) and the sample. The percent removal was calculated as shown in Equation 1, below.

$P_{A}(\%)=\frac{C_{S}-C_{C}}{C_{C}} \times 100,($ Equation 1)

Where:

$\mathrm{P}_{\mathrm{A}}=$ Percent removal by adsorption $(\%)$

$\mathrm{C}_{\mathrm{S}}=$ Liquid-phase sample concentration $(\mathrm{ppb})$

$\mathrm{C}_{\mathrm{C}}=$ Liquid-phase control concentration $(\mathrm{ppb})$

\subsection{Percent removal by other mechanisms}

In addition to removal by adsorption, some removal of heavy metals also occurred by other mechanisms such as precipitation, NOM and heavy metal complexation, or adsorption to the bottle. The percent difference between the concentration added and the measured concentration of the control sample was used to determine the percent removal, as shown in Equation 2, below. 
$P_{O}(\%)=\frac{C_{C}-C_{i}}{C_{i}} \times 100,($ Equation 2)

Where:

$\mathrm{P}_{\mathrm{O}}=$ Percent removal by other mechanisms $(\%)$

$\mathrm{C}_{\mathrm{C}}=$ Liquid-phase control concentration $(\mathrm{ppb})$

$\mathrm{C}_{\mathrm{i}}=$ Initial liquid-phase concentration added (ppb)

\subsection{Adsorbent-phase concentration}

The amount adsorbed per mass of adsorbent, or the adsorbent-phase concentration, is an important parameter for evaluating adsorbent efficiency. The amount adsorbed, or percent removal, when not normalized by the mass of adsorbent, cannot be used to evaluate the efficiency of an adsorbent, because the amount adsorbed is dependent on the amount of adsorbent added. The adsorbent-phase concentration was calculated as shown in Equation 3, below.

$q_{e}=\frac{C_{c}-C_{S}}{A}($ Equation 3)

Where:

$\mathrm{q}_{\mathrm{e}}=$ amount adsorbed $(\mu \mathrm{g} / \mathrm{mg}$ or $\mathrm{mg} / \mathrm{g})$ 
$\mathrm{A}=$ Adsorbent concentration $(\mathrm{mg} / \mathrm{L})$

$\mathrm{C}_{\mathrm{s}}=$ Liquid-phase sample concentration $(\mu \mathrm{g} / \mathrm{L})$

$\mathrm{C}_{\mathrm{C}}=$ Liquid-phase control concentration $(\mu \mathrm{g} / \mathrm{L})$

\subsection{Langmuir adsorption isotherm}

The Langmuir adsorption isotherm is based on an assumption of a reversible chemical equilibrium between adsorbed adsorbate and free adsorbate. The Langmuir model is appropriate for adsorbents with homogeneous adsorbent surfaces, with fixed adsorption sites, each able to adsorb one molecule of adsorbate. The Langmuir adsorption model can be written as:

$q_{e}=\frac{Q_{M} b C_{s}}{1+b C_{s}},($ Equation 4)

Where:

$\mathrm{q}_{\mathrm{e}}=$ amount adsorbed $(\mathrm{mg} / \mathrm{g})$

$\mathrm{Q}_{\mathrm{M}}=$ maximum adsorbent-phase concentration $(\mathrm{mg} / \mathrm{g})$

$\mathrm{b}=$ Langmuir adsorption constant $(\mathrm{L} / \mu \mathrm{g})$

$\mathrm{C}_{\mathrm{s}}=$ Liquid-phase sample concentration (ppb) 
The Langmuir adsorption isotherm can be linearized as shown in Equation 5, below.

$\frac{C_{S}}{q_{e}}=\frac{1}{b Q_{M}}+\frac{C_{S}}{Q_{M}}$, (Equation 5)

\subsection{Freundlich adsorption isotherm}

The Freundlich adsorption isotherm model can be used to describe adsorption for heterogeneous adsorbents, where adsorption sites are not energetically equal. The Freundlich adsorption model is given by:

$q_{e}=K C_{s}^{1 / n},($ Equation 6)

Where:

$\mathrm{q}_{\mathrm{e}}=$ amount adsorbed $(\mathrm{mg} / \mathrm{g})$

$\mathrm{K}=$ Freundlich adsorption capacity parameter $(\mathrm{mg} / \mathrm{g})(\mathrm{L} / \mu \mathrm{g})^{1 / \mathrm{n}}$

$1 / \mathrm{n}=$ Freundlich adsorption intensity parameter (unitless)

$\mathrm{C}_{\mathrm{s}}=$ Liquid-phase sample concentration (ppb)

The Freundlich isotherm can be linearized as shown in Equation 7, below.

$\log \left(q_{e}\right)=\log (K)+\left(\frac{1}{n}\right) \log \left(C_{s}\right),($ Equation 7) 


\subsubsection{Adsorption kinetics for adsorption of lead onto NIP}

Experiments to establish the kinetics of adsorption were conducted in deionized water. The lead standard solution with $1000 \mathrm{mg} / \mathrm{L}$ of lead in nitric acid was diluted to $10,000 \mathrm{ppb}$ and adjusted to $\mathrm{pH} 4$ prior to addition to the sample tubes. Dilution water was created by acidifying deionized water to $\mathrm{pH}$ 4. Samples were prepared by filling $15 \mathrm{~mL}$ centrifuge tubes with $500 \mathrm{ppb}$ of lead and $50 \mathrm{mg} / \mathrm{L}$ NIP, using the acidified deionized water for dilution. The samples were placed on the shaker table and mixed. Samples were taken in duplicate following $1 \mathrm{~h}, 2 \mathrm{~h}, 5 \mathrm{~h}, 24 \mathrm{~h}, 2$ day, 5 day, and 7 day mixing periods. They were centrifuged and treated as outlined above for river water and wastewater.

\subsubsection{Isotherm experiments for adsorption of lead onto NIP}

Lead isotherms were created following the procedure outlined above for kinetic experiments except that the NIP concentration was varied $(10 \mathrm{mg} / \mathrm{L}, 20 \mathrm{mg} / \mathrm{L}, 30 \mathrm{mg} / \mathrm{L}, 40$ $\mathrm{mg} / \mathrm{L}, 50 \mathrm{mg} / \mathrm{L}, 100 \mathrm{mg} / \mathrm{L}, 200 \mathrm{mg} / \mathrm{L}$, and $500 \mathrm{mg} / \mathrm{L}$ ) and the mixing time was kept constant at 14 days.

\subsubsection{Isotherm experiments for adsorption of a mixture of heavy metals onto NIP}

Isotherms were also created for a mixture of lead, copper, zinc, and nickel. The procedure outlined above for lead isotherms was followed except that $500 \mathrm{ppb}$ each of lead, copper, zinc, and nickel were added. 


\subsection{COMPETITIVE EFFECTS OF HUMIC ACID AND WASTEWATER ON ADSORPTION OF METHYLENE BLUE DYE BY ACTIVATED CARBON AND NON-IMPRINTED POLYMERS}

\subsubsection{Adsorbents}

\subsubsection{Activated Carbon}

Three powder activated carbon samples were obtained from Cabot Norit: Norit PAC 200, Darco KB-M, and Darco S-51 (Marshall, TX). Additional information from the manufacturer is available in Appendix C.

\subsubsection{NIP Synthesis}

NIP were made as described in Section 4.1, above.

\subsubsection{Adsorbent characterizations}

BET surface area, average pore size, pore volume, and mesopore volume were measured by Engineering Performance Solutions (Jacksonville, FL). Barret-Joyner-Halenda (BJA) and Quenched Solid Density Functional Theory (QSDFT) analyses were performed using a NOVA BET surface analyzer. The NOVA BET surface analyzer measures the pore volume of the adsorbent as a function of the partial pressure of nitrogen. A sample cell was submerged in liquid nitrogen to maintain a constant temperature of $273 \mathrm{~K}$. The sample cell was slowly filled with nitrogen gas, and the volume of gas was recorded for several pressure intervals to create isotherms. From the isotherm data, the BET surface area, pore volume, average pore size, and mesopore volumes were calculated. The micropore volume was then calculated by subtracting the mesopore volume from the total pore volume, and 
the ratio of the micropore volume to the mesopore volume was calculated by dividing these two values.

BET surface area was calculated by estimating the number of molecules of nitrogen required to cover the surface of the adsorbent in a monolayer. If the number of adsorbate molecules and the size of those molecules are known, they can be multiplied to calculate the surface area of the adsorbent (Quantachrome Corporation, 2013).

The total pore volume was calculated from the total volume of nitrogen gas adsorbed and the density of the nitrogen gas at $273 \mathrm{~K}$. The average pore size was calculated from the total pore volume using the $\mathrm{BJH}$ and QSDFT methods for macropores and micropores, respectively.

The mesopore volume was calculated based on the BJH method. The BJH method was the earliest method developed to estimate the pore size distribution for activated carbons, and assumes cylindrical pores (Eduardo and Tascon, 2008). In the BJH method, isotherm data consisting of adsorbed nitrogen gas volumes versus pressures, are matched mathematically using software to those expected from a certain pore distribution (Quantachrome Corporation, 2013).

The QSDFT method, more accurately, accounts for surface roughness and heterogeneity (Quantachrome Corporation, n.d.). The QSDFT method was used to calculate the micropore volume and the BJH and QSDFT methods were combined to calculate the 
average pore radius. Both methods use isotherms of adsorbed nitrogen gas volumes versus pressures, which are matched using software and mathematical analysis to those expected from a certain pore distribution and shape. As the adsorbate pores are filled with nitrogen, and knowing the density of the nitrogen gas, the volume occupied by the gas can be calculated. The hysteresis during adsorption and desorption can be matched to a specific pore distribution (Quantachrome Corporation, n.d.).

\subsubsection{Humic acid}

A Suwannee River Humic Acid sample (Suwanee River Humic Acid II) was purchased from the International Humic Substances Society (St. Paul, MN). A chemical characterization of the functional groups in the humic acid are provided in Table 4-2 below.

Table 4-2: Chemical properties and functional groups for Suwanee River II Humic Acid

\begin{tabular}{|l|l|l|l|l|l|l|l|l|l|l|}
\hline \multicolumn{2}{|l|}{ Chemical Characterization (International Humic Substances Society, 2015a) } \\
\hline C (\%) & $\mathrm{H}(\%)$ & $\mathrm{O}(\%)$ & $\begin{array}{l}\mathrm{N} \\
(\%)\end{array}$ & $\begin{array}{l}\mathrm{S} \\
(\%)\end{array}$ & $\mathrm{P}(\%)$ & & & & \\
\hline 52.63 & 4.28 & 42.04 & 1.17 & 0.54 & 0.013 & & & & \\
\hline Functional Groups & International Humic Substances Society, 2015b) $* *$ & $\mathrm{~N}$ & $\mathrm{RMSE}$ \\
\hline Carboxyl & Phenolic & $\mathrm{Q}_{1}$ & $\begin{array}{l}\text { Log } \\
\mathrm{k}_{1}\end{array}$ & $\mathrm{n}_{1}$ & $\mathrm{Q}_{2}$ & $\begin{array}{l}\text { Log } \\
\mathrm{k}_{2}\end{array}$ & $\mathrm{n}_{2}$ & $\mathrm{~N}$ & & \\
\hline 9.13 & 3.72 & 9.74 & 4.35 & 3.30 & 4.48 & 10.44 & 1.73 & 171 & 0.0815 \\
\hline
\end{tabular}

\footnotetext{
** The carboxyl functional groups are represented by the charge density (meq $/ \mathrm{gC})$ at $\mathrm{pH} 8$. The phenolic functional groups are measured by the change in charge density between $\mathrm{pH} 8$ and $10 . \mathrm{Q}_{1}$ and $\mathrm{Q}_{2}$ are the maximum charge densities of the carboxyl and phenolic functional groups, respectively, and $\log \mathrm{k}_{1}$ and $\log \mathrm{k}_{2}$ are the mean $\log \mathrm{k}$ values for binding of protons. The empirical parameters, $\mathrm{n}_{1}$ and $\mathrm{n}_{2}$, describe the width of the variability of the Log $\mathrm{k}$ values. $\mathrm{N}$ is the number of points used for the titration, and RMSE is the root of the mean square error for fitting the data to a Langmuir-Freundlich model.
} 


\subsubsection{Wastewater}

Secondary wastewater effluent was collected from the Robert O. Pickard Environmental Centre in Ottawa, Ontario. It was stored in the refrigerator and then left at room temperature overnight prior to use.

\subsubsection{Single-solute adsorption}

Adsorption isotherm tests were conducted to evaluate removal of methylene blue (MB) dye from deionized water using NIP, Norit PAC 200, Darco KB-M, and Darco S-51. 150 $\mathrm{mL}$ amber glass bottles were filled with $50 \mathrm{~mL}$ of deionized water spiked with $10,12,14$, 16,18 or $20 \mathrm{ppm}$ of MB dye. $50 \mathrm{~g} / \mathrm{L}$ solutions were prepared for the NIP three activated carbons, and sonicated (Vibracell Sonics, Sonics and Materials Inc., Newtown Connecticut) for 15 minutes to disperse the particles. $0.5 \mathrm{~mL}$ of the NIP solution or $0.1 \mathrm{~mL}$ of each activated carbon solution was then added to each sample bottle to obtain final concentrations of $0.1 \mathrm{~g} / \mathrm{L}$ for the activated carbons and $0.5 \mathrm{~g} / \mathrm{L}$ for the NIP. The samples were mixed on a shaker table (New Brunswick Scientific Excella E1 Platform Shaker, New Brunswick Scientific Inc.) for two weeks. They were then centrifuged (Thermo Scientific Sorval Legend RT+, Fisher Scientific) for 1 hour at a speed of 10,000 rpm $(670,800 \mathrm{~g})$.

Following centrifugation, the centrate was poured into $15 \mathrm{~mL}$ centrifuge bottles and analyzed with a UV-visible spectrophotometer (Varian Model Cary 100Bio; Victoria, Australia) using a scan from 200 to $800 \mathrm{~nm}$. A peak for MB was identified at $663 \mathrm{~nm}$, which did not experience any interference from humic acid or wastewater, and was used for data analysis. PH was not monitored. 


\subsubsection{Simultaneous loading}

Isotherm experiments were conducted using $\mathrm{MB}$ in a $20 \mathrm{mg} / \mathrm{L}$ humic acid solution and secondary wastewater effluent. Samples were adjusted to $\mathrm{pH} 7$ using sodium hydroxide and hydrochloric acid prior to addition of MB. The procedure outlined above for singlesolute adsorption (Section 4.5.5) was then followed using NIP and Norit PAC 200 as adsorbents. Control samples were prepared without adsorbents using the same procedure (including centrifuging) and used to generate calibration curves. PH was not monitored.

\subsubsection{Pre-loading}

Isotherm experiments were also conducted using NIP and Norit PAC 200 that were first exposed to humic acid or wastewater solutions and then subsequently exposed to MB. 50 $\mathrm{mL}$ of the $20 \mathrm{mg} / \mathrm{L}$ humic acid solution or secondary wastewater effluent were added to $150 \mathrm{~mL}$ amber glass bottles. NIP or Norit PAC 200 were added to obtain final concentrations of $0.5 \mathrm{~g} / \mathrm{L}$ or $0.1 \mathrm{~g} / \mathrm{L}$, respectively. The bottles were placed on a shaker table (New Brunswick Scientific Excella E1 Platform Shaker; New Brunswick Scientific Inc.) for 24 hours. Following the 24 hour adsorption period, the samples were centrifuged (Thermo Scientific Sorval Legend RT+; Fisher Scientific) in $50 \mathrm{~mL}$ centrifuge tubes for 1 hour at $10,000 \mathrm{rpm}(670,800 \mathrm{~g}) .50 \mathrm{~mL}$ of deionized water with methylene blue ranging from 10-20 ppm was added to the centrifuge tubes and they were shaken to disperse the particles. The solution was then poured into $150 \mathrm{~mL}$ amber glass bottles, shaken on a platform shaker for two weeks, and centrifuged, and the centrate was analyzed using UV visible spectrophotometry. PH was not monitored. 


\subsubsection{Statistical analysis}

To determine whether or not there was a competitive effect from humic acid or wastewater during either pre-loading or simultaneous adsorption, comparisons between sets of data for different sample conditions were necessary. Since the MB concentration was also varied for each data set, a t-test using a paired comparison of means was used. First, the differences between the average adsorption capacities for the two conditions being compared were calculated for each $\mathrm{MB}$ concentration. For example, to investigate the effect of competition from wastewater, the average adsorption capacity in wastewater for each MB concentration (10ppm-20ppm) was subtracted from the corresponding adsorption capacity in deionized water. The average and variance of these differences were calculated, and used to calculate the corresponding t-statistics and estimated probabilities for rejecting the null hypothesis. The null hypothesis was that there was no difference in adsorption capacity between the two sample groups. If the $\mathrm{P}$ value was greater than 0.1 , which corresponds to a $90 \%$ confidence limit, the difference was considered insignificant. If it was less than 0.1 , the difference was considered significant. The percent difference between the samples was calculated similarly using a paired t-test with a $90 \%$ confidence interval. The percent difference was calculated by dividing the calculated difference in adsorption capacities for the two samples by the average of the two samples and multiplying by 100 . The average of the two samples was used rather than the amount adsorbed for deionized water, because some of the comparisons were done between samples other than deionized water. 


\subsubsection{Zeta potential}

\subsubsection{Sample preparation}

\subsection{Adsorbents in water}

$5 \mathrm{mg}$ of Norit PAC 200 or $25 \mathrm{mg}$ of NIP were weighed and added to $50 \mathrm{~mL}$ centrifuge tubes containing $50 \mathrm{~mL}$ each of deionized water. The solutions were sonicated (Vibracell Sonics, Sonics and Materials Inc., Newtown Connecticut) to disperse the particles.

\subsection{Adsorbents in humic acid}

$200 \mathrm{~mL}$ of $20 \mathrm{mg} / \mathrm{L}$ humic acid solution were prepared by adding $4 \mathrm{mg}$ of Suwanee River Humic Acid to $200 \mathrm{~mL}$ of deionized water. This solution was also used for pre-loading of adsorbents with humic acid as outlined in Section 4.5.9.1.3, below. $50 \mathrm{~mL}$ of this solution was added to each of two $50 \mathrm{~mL}$ centrifuge tubes. $5 \mathrm{mg}$ of Norit PAC 200 or $25 \mathrm{mg}$ of NIP were weighed and added to each tube. The solutions were sonicated for 15 minutes each to disperse the particles.

\subsection{Pre-loading of adsorbents with humic acid}

The procedure outlined in Section 4.5.9.1.2, above, was followed to create solutions with PAC 200 and NIP in $20 \mathrm{mg} / \mathrm{L}$ of humic acid. The centrifuge tubes were placed on a shaker table (New Brunswick Scientific Inc.) for 24 hours. They were then centrifuged (Thermo Scientific Sorval Legend $\mathrm{RT}^{+}$, Fisher Scientific) for 1 hour at a speed of $10,000 \mathrm{rpm}$ $(670,800 \mathrm{~g})$. Following centrifugation, the centrate was poured out and $50 \mathrm{~mL}$ of deionized

water was added to each centrifuge tube. Some of the centrate remained in the tube, which 
was necessary to avoid any loss of the particles, but care was taken to minimize the amount remaining. The solutions were sonicated for 15 minutes each to disperse the particles in the deionized water.

\subsubsection{Zeta potential measurements and instrument}

A Zeta Meter 4.0 (Zeta-Meter Inc., Staunton, Virginia) was used for zeta potential measurements. The Zeta Meter uses backlighting to increase the visibility of small particles that would usually require high magnification. The magnification provided by the objectives and eyepieces was 80x (Zeta Meter Inc, 2010). First, the electrophoresis cell was filled with the sample. Then the Zeta Meter was turned on. The electrophoresis cell was placed on the cell holder and the electrodes were connected. The specific conductance of the sample was measured and set with the Zeta Meter. A voltage of $300 \mathrm{~V}$ was applied. The time for a particle to travel between two tracking lines was measured by pressing the track key as the particle reached the first tracking line and releasing it as the particle reached the second tracking line. This was repeated for 20 particles. The instrument automatically calculated the average zeta potential and standard deviation. The electrophoresis cell was rinsed well with tap water followed by the next sample between samples.

\subsection{INVESTIGATION OF PHYSICAL SEPARATION PROCESSES FOR REMOVAL OF NON-IMPRINTED POLYMERS FOLLOWING WATER AND WASTEWATER TREATMENT}

\subsubsection{Preparation of NIP solution}

NIP were used for all removal experiments because they are smaller than the MIP, meaning that they should theoretically be more difficult to remove and present a most-challenging 
scenario. They are also easier to prepare because they do not require the addition and subsequent removal of a template molecule. An NIP solution was prepared by sonicating the NIP (Vibracell Sonics, Sonics and Materials Inc., Newtown Connecticut) in 1 L of water for 15 minutes to disperse the NIP before being diluted to a final concentration of 5 mg/L. The solution was stirred thoroughly before being dispensed.

\subsubsection{Removal of NIP with gravity settling}

To determine the settleability of NIP with no treatment, three $1 \mathrm{~L}$ beakers of the $5 \mathrm{mg} / \mathrm{L}$ NIP suspension in tap water were monitored for 24 hours. Samples were taken $5 \mathrm{~cm}$ below the surface of the water and measured for turbidity.

\subsubsection{Removal of NIP with filtration}

A lab-scale filtration set-up was used to evaluate the effectiveness of varying filter pore sizes on NIP removal. The pore sizes investigated were $0.22 \mu \mathrm{m}$ (using a nylon syringe filter from Derian); $0.7 \mu \mathrm{m}$ (GF/F glass microfiber filters, Whatmann); $1 \mu \mathrm{m}$ (GF/B glass microfiber filters, Whatmann); $1.6 \mu \mathrm{m}$ (G6 glass fiber circles, Fisher); and $3 \mu \mathrm{m}$ (Grade 6 qualitative filters, Whatmann).

\subsubsection{Removal of NIP with centrifugation}

Centrifuge time and speed were varied to determine optimum conditions for removal of NIP. The NIP solution was prepared as outlined above and poured into three $50 \mathrm{~mL}$ tubes. The centrifuge (Thermo Scientific Sorval Legend $\mathrm{RT}^{+}$, Fisher Scientific) was run for varying times and speeds. Then a $20 \mathrm{~mL}$ sample was pipetted from the surface. In the first 
set of experiments, the centrifuge time was varied for a constant speed of 10,000 rpm corresponding to 670,800 times the force of gravity $(\mathrm{g})$. In the second set of experiments the centrifuge speed was varied for a constant time.

\subsubsection{Removal of NIP with ballasted flocculation}

The jar test procedure for ballasted flocculation was followed as described by Desjardins et al (2001). A jar test apparatus from Phipps and Bird (Richmond, Virginia) was used with a flat blade impeller. The mixing speed was kept constant throughout and the coagulants, microsand, and polymer were added as follows: (1) at time $t=0$, the coagulant was added and the jar tester started; (2) at time $\mathrm{t}=2$, the microsand and a percentage of the polymer were added; (3) at time $t=4$, the remainder of the polymer was added; (4) at time $t=10$ the mixing was turned off and the NIP were allowed to settle by gravity; and (5) at time $\mathrm{t}=13$, a sample was taken approximately $5 \mathrm{~cm}$ below the surface. The times were varied in some cases to achieve optimum performance but the order of the additions was maintained. The speed of impeller rotation for the jar-tester remained constant throughout (Desjardins et al., 2002).

\subsubsection{Chemicals and sand for ballasted flocculation}

The coagulant used for ballasted flocculation was alum (CAS 7784-31-8) (Anachemia, Montreal, Quebec). The Polymer was Flopolymer CA4800 (SNF, Trois Rivieres, Quebec). Microsand samples were obtained from John Meunier Inc. (Montreal, Quebec). 


\subsubsection{Effect of settling time}

To illustrate the effect that ballasted flocculation had on NIP settleability, the NIP solution was allowed to settle for $1 \mathrm{~h}$ following treatment and the turbidity was monitored with time.

\subsubsection{Control parameters}

Since there were many factors (alum dose, polymer dose, the percent of the polymer added initially, sand dose, mixing intensity, coagulation time, flocculation time, and settling time) capable of influencing the effectiveness of the ballasted flocculation treatment, a statistical approach employing a $2^{8-4}$ factorial design was used to determine which factors had a significant impact on performance. The combinations of conditions tested are outlined in Table 4-3, below. A linear regression model was created in Excel from the results.

Table 4-3: Ballast flocculation control parameters

\begin{tabular}{|l|l|l|l|l|l|l|l|}
\hline & $\begin{array}{l}\text { polymer } \\
\text { percent } \\
\text { added } \\
\text { in Step } \\
2\end{array}$ & $\begin{array}{l}\text { alum dose } \\
\text { solym } \\
\text { sose } \\
\text { dose } \\
(\mathrm{mL})\end{array}$ & $\begin{array}{l}\text { mixing } \\
\text { intensity } \\
\text { (rpm) }\end{array}$ & $\begin{array}{l}\text { loagulation } \\
\text { time (min) }\end{array}$ & $\begin{array}{l}\text { flocculation } \\
\text { time (min) }\end{array}$ & $\begin{array}{l}\text { settling } \\
\text { time } \\
(\mathrm{min})\end{array}$ \\
\hline 5 & 2 & 70 & 2 & 300 & 4 & 10 & 3 \\
\hline 10 & 2 & 30 & 2 & 150 & 4 & 6 & 6 \\
\hline 10 & 10 & 30 & 4 & 150 & 2 & 6 & 6 \\
\hline 5 & 2 & 30 & 2 & 150 & 2 & 10 & 3 \\
\hline 10 & 10 & 30 & 2 & 300 & 4 & 6 & 3 \\
\hline 10 & 10 & 70 & 2 & 150 & 2 & 10 & 3 \\
\hline 5 & 10 & 70 & 2 & 150 & 4 & 6 & 6 \\
\hline 10 & 10 & 70 & 4 & 300 & 4 & 6 & 6 \\
\hline
\end{tabular}




\begin{tabular}{|l|l|l|l|l|l|l|l|}
\hline 10 & 2 & 30 & 4 & 300 & 2 & 6 & 3 \\
\hline 5 & 2 & 70 & 2 & 150 & 2 & 10 & 6 \\
\hline 10 & 2 & 70 & 2 & 300 & 2 & 6 & 6 \\
\hline 5 & 10 & 30 & 2 & 300 & 2 & 10 & 6 \\
\hline 5 & 10 & 30 & 4 & 150 & 4 & 10 & 3 \\
\hline 5 & 10 & 70 & 4 & 300 & 2 & 10 & 3 \\
\hline 10 & 2 & 70 & 4 & 150 & 4 & 6 & 3 \\
\hline 5 & 2 & 30 & 4 & 300 & 4 & 10 & 6 \\
\hline
\end{tabular}

\subsubsection{Optimization of ballasted flocculation conditions}

Using the linear regression model obtained from the factorial design experiments, a path proportional to the coefficients in the regression model was followed until an increase in turbidity was observed. The conditions corresponding to the step before the increase in turbidity were taken as the optimum conditions.

\subsubsection{Effect of alum dose}

To compare the effectiveness of ballasted flocculation for tap water and wastewater, the effect of varying alum dose on ballasted flocculation was compared for both. The procedure for ballasted flocculation was followed as outlined above. For tap water, the alum was dispensed from a $1 \mathrm{~g} / \mathrm{L}$ stock solution and for wastewater a $10 \mathrm{~g} / \mathrm{L}$ stock solution was used due to the higher alum concentrations required for wastewater. The wastewater used was secondary effluent collected from the Robert O. Pickard Environmental Centre in Ottawa, Ontario. 


\subsubsection{Scanning electron microscope (SEM) images}

A Tescan Vegall XMU SEM instrument was used to obtain SEM images. The NIP were coated with gold prior to imaging using RF sputter (Anatech Hummer, Union City, CA, USA). The images were collected at a working distance of 7-8mm.

\subsubsection{Evaluation of removal efficiency with various analytical devices}

\subsubsection{Turbidity measurements}

The amount of NIP removed was evaluated using before and after comparisons of turbidity. Turbidity measurements were taken using a Hach 2100 AN turbidity meter.

\subsubsection{Particle size and distribution as measured by the DPA 4100 particle analysis system}

A DPA 4100 Particle Analysis System (Brightwell Technologies, Ottawa, ON) was used to obtain all particle count measurements. The DPA 4100 Particle Analysis system analyzes samples which are passed through a flow cell capillary device while light is shone on them. The resulting signal is optically magnified and projected onto a photo diode array detector (Thomas and Moore, 2004). It can quickly and accurately provide particle size distributions and particle concentration measurements, and simultaneously gathers images of the sample stream, allowing for characterization of particle types. $10 \mathrm{~mL}$ samples were stirred continuously as $2 \mathrm{~mL}$ sub-samples were drawn through the flow cell capillary and injected into the sampling syringe at a flowrate of $0.22 \mathrm{~mL} / \mathrm{min}$. Particle size distributions were measured, and images of the sample water were gathered. Results reported are the 
average of two experimental replicates each of which consisted of three analytical replicates.

\subsubsection{Particle size as measured by the Nano ZS Zetasizer}

A Malvern Nano ZS Zetasizer was used to obtain particle size distributions and measure the average particle size before and after NIP removal. Two experimental replicates each consisting of two analytical replicates were conducted. The Nano ZS Zetasizer uses light scattering to measure the size of particles in a solution. A laser is directed towards the sample cell, and the light scattered at $173^{\circ}$ is measured. This angle means that the light does not have to travel through the entire sample, so multiple scattering, where light that has already been scattered by one particle hits another particle, as well as interference from dust and other contaminants, is avoided. Dust particles are usually large, and tend to scatter in a forward direction, and would not be detected at this angle (Malvern Instruments, 2000). Measurements taken using the Nano ZS Zetasizer complimented those provided by the DPA 4100 Particle Analysis System because the Nano ZS Zetasizer can measure particle sizes from $0.3 \mathrm{~nm}$ to $10 \mu \mathrm{m}$ in diameter, whereas the DPA 4100 does not accurately measure particles below $2 \mu \mathrm{m}$ in diameter. The Zetasizer was able to provide accurate size distributions before and after NIP removal, but could not provide particle concentrations, which were provided by the DPA 4100.

\subsubsection{Exposure of NIP to acidic and basic conditions}

$20 \mathrm{mg}$ samples of NIP were added to $1.5 \mathrm{~mL}$ centrifuge tubes and $1 \mathrm{~mL}$ of water of varying $\mathrm{pH}(2-11)$ was added to each tube. The $\mathrm{pH}$ solutions were prepared using deionized water 
and concentrated sodium hydroxide or hydrochloric acid solutions (18 \% and 36.5-38\%, respectively). The tubes were shaken to mix and left for 24 hours before the NIP were centrifuged and dried at room temperature.

\subsection{REFERENCES}

Certificate of Accreditation - Canadian Association for Laboratory Accreditation. 2010.

Desjardins, C., B. Koudjonou, and R. Desjardins. 2002 Laboratory Study of Ballasted Flocculation. Water Research, 36 (3), 744-54.

Eduardo, B., and J. Tascon. 2008 Classical Methods for Pore Characterization. Adsorption by Carbons: Novel Carbon Adsorbents,. Elsevier, New York.

Englert, B. (US E. 2007 Method 1694 : Pharmaceuticals and Personal Care Products in Water, Soil, Sediment, and Biosolids by HPLC / MS / MS. USEPA 821-R-08002,. Washington, D.C.

International Humic Substances Society. 2015a Elemental Compositions and Stable Isotopic Ratios of IHSS Samples.

International Humic Substances Society. 2015b Acidic Functional Groups of IHSS Samples.

Malvern Instruments. 2000 Dynamic Light Scattering: An Introduction in 30 Minutes. Http:/Www.Malvern.Com/En/Products/Technology/Dynamic-Light-Scattering/, $1-8$.

Pelekani, C., and V. . Snoeyink. 1999 Competitive Adsorption in Natural Water: Role of Activated Carbon Pore Size. Water Research, 33 (5), 1209-1219.

Quantachrome Corporation. 2013 Nova e Series Brochure. Boynton Beach. 
Quantachrome Corporation. n.d. Application of QSDFT (Quenched Solid Density Functional Theory) - A Novel Density Functional Theory for an Accurate Pore Size Analysis of Disordered Porous Carbons. Powder Technote, 40.

Thomas, D., and P. Moore. 2004 Water Treatment Plant Particle Characterization Using Digital Imaging Technology (April), 1-10.

Wei, S., A. Molinelli, and B. Mizaikoff. 2006 Molecularly Imprinted Micro and Nanospheres for the Selective Recognition of 17Beta-Estradiol. Biosensors \& Bioelectronics, 21 (10), 1943-51.

Yoon, Y., P. Westerhoff, S. a. Snyder, and M. Esparza. 2003 HPLC-Fluorescence Detection and Adsorption of Bisphenol A, 17 $\beta$-Estradiol, and 17 $\alpha$-Ethynyl Estradiol on Powdered Activated Carbon. Water Research, 37 (14), 3530-3537. Zeta Meter Inc. 2010 Zeta Meter System 4.0 Operating Instructions. Staunton VA. 


\title{
CHAPTER 5: USE OF NON-IMPRINTED POLYMERS FOR REMOVAL OF ENDOCRINE DISRUPTING COMPOUNDS AND PHARMACEUTICALS FROM WATER AND WASTEWATER
}

\author{
Audrey Murray ${ }^{1}$, Banu Örmeci ${ }^{1}$, and E.P.C. Lai ${ }^{2}$ \\ ${ }^{1}$ Department of Civil and Environmental Engineering, Carleton University, 1125 Colonel \\ By Drive, Ottawa ON K1S 5B6, Canada. \\ ${ }^{2}$ Department of Chemistry, Carleton University, 1125 Colonel By Drive, Ottawa ON K1S \\ 5B6, Canada.
}

\subsection{ABSTRACT}

Endocrine disrupting compounds (EDCs) and pharmaceuticals pose a challenge for water and wastewater treatment because they exist at very low concentrations in the presence of competing substances at much higher concentrations. Non-imprinted polymers (NIP) were tested to evaluate their potential as a treatment for EDCs including: 17- $\beta$ estradiol (E2), 17 $-\alpha$ ethinylestradiol (EE2), estrone (E1), bisphenol A (BPA), and diethylstilbestrol (DES) as well as a group of 12 pharmaceuticals. NIP were able to remove $98 \%$ of spiked E2, $80 \%$ of EE2, 87\% of BPA, and up to $97 \%$ of DES from single-solute solutions. For a $0.5 \mathrm{ppm}$ mixture of E2, EE2, E1, BPA, and DES, the minimum removal was $24 \%$ (E2) and the maximum was $49 \%$ (DES). They were also able to remove 9 out of 12 of the pharmaceuticals to some degree from deionized water and 3 out of 7 of the pharmaceuticals measured in wastewater. Overall, NIP are a promising advanced treatment for removal of 
both EDCs and pharmaceuticals, but further research is necessary to determine their full potential.

\subsection{INTRODUCTION}

Pharmaceuticals and endocrine disrupting compounds (EDCs) are two overlapping classes of micropollutants of emerging concern for water and wastewater treatment due to their ability to cause adverse health effects at very low concentrations. Pharmaceuticals are designed to target a wide range of health concerns and thus cause a variety of health effects when taken inadvertently, and the transformation products of those parent compounds (Evgenidou et al., 2015), and effects of mixtures of pharmaceuticals and other chemicals (Kuzmanovic' et al., 2014) remain largely unknown. Some pharmaceuticals are also EDCs, but EDCs also include some chemicals which are not pharmaceuticals, such as bisphenol A and atrazine. EDCs mimic or block the hormonal systems of both humans and animals. They can cause reproductive, immune, hormonal, and neurobehavioral disorders (Yang et al., 2006) as well as breast, testicular, and prostate cancers (Gillesby and Zacharewski, 1998; Ashby et al., 1997). There is some debate about the concentrations of EDCs that lead to health effects. For example, Snyder et al (2010) suggested that a screening level acceptable daily intake of $17-\beta$ estradiol should be $0.000026 \mu \mathrm{g} / \mathrm{kg} /$ day, while the Australian guidelines recommend an acceptable daily intake of $0.05 \mu \mathrm{g} / \mathrm{kg} / \mathrm{day}$ (Anderson et al., 2010).

Water and wastewater treatment plants were originally designed to remove particles, organic matter and nutrients, and thus they are not very effective in removing 
micropollutants. In addition, micropollutants pose a challenge for water and wastewater treatment because of competition from other pollutants present at much higher concentrations. A sizeable portion of the capacity of various treatment processes is consumed treating these higher concentration pollutants in addition to some micropollutants. Thus, targeted treatment methods with specific preferences for micropollutants are needed. Molecularly imprinted polymers (MIP) are one such potential treatment. MIP are polymers formed in the presence of a template molecule and possess an affinity for that template molecule once polymerization is complete and the template has been removed. MIP have functional groups capable of interacting with the template molecule through hydrogen bonding, Vander Waals forces, etc. Typically, the template will form several interactions with the MIP functional groups, and the spatial arrangement of those interactions will be unique to the template, providing the MIP with a specific affinity for the template molecule (Masqué, 2001). Non-imprinted polymers (NIP) are prepared in the same way, but in the absence of a template molecule, and are less specific than MIP. Thus, NIP are simple to prepare and do not require the washing steps required by MIP.

A preference for micropollutants is advantageous to target them directly without interference from higher-concentration pollutants. On the other hand, there are a wide range of micropollutants which need to be targeted, so designing treatments to target a single compound may not be useful. For this reason, researchers have investigated the simultaneous synthesis of MIP with several different templates for targeted treatment of micropollutants. Dai et al (2012), simultaneously created MIP with templates to target 
ibuprofen, naproxen, ketoprofen, diclofenac, and clofibric acid (Dai et al., 2012); and Venkatesh et al (2014), created MIP for nicotine, epinephrine, and physostigmine (Venkatesh et al., 2014). A potential downside to this approach is that, these are only a small subset of the micropollutants of interest during water and wastewater treatment, and this technique requires the identification and intentional targeting of individual micropollutants. In addition, using multiple templates substantially increases the complexity and cost of preparing MIPs.

Another disadvantage of MIP is that their specificity is dependent on the shape of the cavity remaining following removal of the template. The cavity shape can change due to the swelling properties of MIP in different solvents, and since MIP are difficult to synthesize in aqueous solutions, their specificity is typically low in water. In some cases, NIP can adsorb equal amounts or even more of the template molecule from water (Deng et al., 2009; Meng et al., 2012). Accordingly, NIP may be considered as a treatment method for micropollutants in their own right (Murray and Ormeci, 2012).

This research provides an investigation into the effectiveness of NIP for removal of a wide range of EDCs and pharmaceuticals in both deionized water and wastewater. NIP were evaluated for their ability to remove 5 EDCs: $17-\beta$ estradiol (E2), 17- $\alpha$ ethinylestradiol (EE2), estrone(E1), diethylstilbestrol (DES) and bisphenol A (BPA) from deionized water. Experiments were conducted with each EDC individually, and with a mixture of the five EDCs. The kinetics of adsorption were also tested for the mixture of 5 EDCs. Additionally, a mixture of 12 pharmaceuticals: acetaminophen, caffeine, carbamazepine, cloxacillin, 
diphenhydramine, enrofloxacin, lincomycin, oxacillin, sulfadiazine, sulfamethizole, sulfanilamide, and sulfathiazole was studied both in deionized water and wastewater. NIP are easy and cost effective to prepare in comparison to MIP, can be manufactured in large quantities, and have the potential to remove a wide variety of micropollutants including both EDCs and pharmaceuticals, which gives them excellent potential for application in full-scale water and wastewater treatment.

\subsection{MATERIALS AND METHODS}

\subsubsection{Micropollutants}

Endocrine disrupting compounds (EDCs) and pharmaceuticals: 17- $\beta$ estradiol (E2), 17- $\alpha$ ethinylestradiol (EE2), estrone(E1), diethylstilbestrol (DES), bisphenol A (BPA), acetaminophen, caffeine, carbamazepine, cloxacillin, diphenhydramine, enrofloxacin, lincomycin, oxacillin, sulfadiazine, sulfamethizole, sulfanilamide, and sulfathiazole were all purchased from Sigma-Aldrich (St Louis, Missouri).

$1 \mathrm{mg} / \mathrm{mL}$ stock solutions were prepared in methanol and stored in the freezer prior to use.

\subsubsection{Analytical measurements}

\subsubsection{Single EDC analysis}

Analysis was conducted using high-performance liquid chromatography (HPLC) with a Phenomenex 50 x $2.00 \mathrm{~mm}$ PFP column and a mobile phase with 55:45 methanol:deionized water. A Shimadzu LC 20 AD HPLC with a Sil 20A autosampler (Shimadzu; Kyoto, Japan) was used for all analysis. Fluorescence detection (FD) with a RF-10AXL detector 
(Shimadzu; Kyoto, Japan) was used for E2, EE2 and BPA, and photo diode array (PDA) detection with a SPD-M20A detector (Shimadzu; Kyoto, Japan) was used for DES. The limits of detection were $0.1 \mathrm{ppb}$ for E2, EE2 and BPA, and $0.25 \mathrm{ppm}$ for DES.

\subsubsection{Treatment of water with a mixture of EDCs and removal kinetics}

Analysis was conducted using high performance liquid chromatography tandem mass spectrometry (LC/MS/MS) in MRM mode. A Shimadzu HPLC (LC 20 AD) was used in conjunction with an AB Sciex API 2000 triple quadrupole tandem mass spectrometer (Framingham, Massachusetts). The HPLC column used was a Phenomenex Kinetex $2.6 \mu$ PFP column. Electrospray ionization was applied in negative mode for the mass spectrometer. Both positive mode and negative mode were tested and negative mode provided the best results. Calibration curves were generated and used to calculate the concentrations of the EDCs after treatment. The limit of detection was $20 \mathrm{ppb}$ for the five EDCs tested.

\subsubsection{Removal of pharmaceuticals}

Analysis was conducted by Axys Analytical Services (Sidney, BC) using LC/MS/MS (Axys Method MLA-075 Rev 5). The Axys Analytical Services method is certified by the Canadian Association for Laboratory Accreditation and corresponds to USEPA Method 1694(Certificate of accreditation - Canadian Association for Laboratory Accreditation, 2010). The Axys Analytical method uses formic acid to adjust the $\mathrm{pH}$ of the sample to 2 prior to solid phase extraction followed by LC/MS/MS analysis (Englert, 2007). Refer to Appendix A for the reporting limits of the analysis. 


\subsubsection{Identification of important parameters}

Preliminary experiments were performed using E2 as a model compound to investigate the effect of NIP mass, binding time, E2 concentration, and NIP batch on the adsorption of E2 onto NIP. This was necessary to design the future experiments in a way that focused on the most important variables. NIP concentrations of 5, 10, 15, or $20 \mathrm{mg} / \mathrm{L}$; binding times of 5, 7.5 , or 10 minutes; E2 concentrations of 2, 6, 10, or $14 \mathrm{ppm}$; and two separate NIP batches were tested. Due to the large number of conditions tested, replicates were not performed for each combination. Instead, 3 replicates were performed for the following combinations: $8.5 \mathrm{ppm}$ E2 with $5,12.5$, or $20 \mathrm{mg} / \mathrm{L}$ of NIP, and a 7.5 minute contact time, for NIP batch 1 or 2 . These replicates were used to estimate a pooled variance for all of the results.

The tests were conducted by weighing the appropriate mass of NIP into $1.5 \mathrm{~mL}$ plastic centrifuge tubes and adding $1 \mathrm{~mL}$ of deionized water spiked with the selected $\mathrm{E} 2$ concentration. The samples were sonicated (Vibracell Sonics; Sonics and Materials Inc.; Newtown, Connecticut) for the required contact time. Following sonification, the tubes were centrifuged (Sorvall Micro 21; Thermo Scientific; Waltham, Massachusetts) for 5 minutes at $10,000 \mathrm{rpm}$ to remove the NIP. The centrate was then analyzed with HPLC (Shimadzu LC 20 AD; Kyoto, Japan) using fluorescence detection (FD) (Shimadzu RF10AXL; Kyoto, Japan). Removal was calculated by comparison to a control prepared in deionized water, following the same procedure outlined above, but without the addition of NIP. 


\subsubsection{Treatment of water with a single EDC}

NIP were tested for their ability to remove EE2, DES, and BPA from deionized water through a series of batch tests each with a single solute. For each sample, $5 \mathrm{mg}$ of NIP was weighed into a $1.5 \mathrm{~mL}$ centrifuge tube, and $1 \mathrm{~mL}$ of deionized water spiked with the relevant concentration of EE2, DES, or BPA was added. NIP from batch 1 were used so that the results could be compared with those obtained for E2. The tubes were sonicated for 5 minutes to disperse the NIP before centrifugation at $10,000 \mathrm{rpm}$ for 5 minutes. The

centrate with HPLC as previously described. Three experimental replicates each consisting of two analytical replicates were performed. For EE2 and BPA, concentrations were varied between 2-14 ppb, and for DES, concentrations of 5-20 ppm were tested. Experiments were performed at room temperature, but neither temperature nor $\mathrm{PH}$ were monitored.

\subsubsection{Treatment of water with a mixture of EDCs}

NIP were also tested for treatment of a mixture of EDCs consisting of 0.5 ppm each of E2, EE2, E1, BPA, and DES over a 24 hour contact time. NIP concentrations of $0.1,1,10,100$, and $1000 \mathrm{mg} / \mathrm{L}$ were tested to establish the range over which NIP were effective. $200 \mathrm{~mL}$ amber glass bottles were filled with $50 \mathrm{~mL}$ each of deionized water. Next, a concentrated NIP solution was created and sonicated for 15 minutes before it was pipetted into the bottles to achieve the desired final concentration. Then, a mixture of E2, EE2, E1, BPA, and DES, each with a concentration of $25 \mathrm{ppm}$, was created in methanol and $1 \mathrm{~mL}$ was pipetted into each of the bottles, to obtain a final concentration of $0.5 \mathrm{ppm}$ for each EDC. The bottles were placed on a shaker table (Excella E1 Platform Shaker; New Brunswick Scientific Inc; 
Enfield, Connecticut) for 24 hours. The following day, the samples were centrifuged at $10,000 \mathrm{rpm}(670,800 \mathrm{~g})$ for 1 hour (Sorval Legend RT+; Thermo Scientific/Fisher Scientific; Waltham, Massachusetts), and the centrate was analyzed with LC/MS/MS as previously described. Three experimental replicates, each consisting of 6 injection replicates, were conducted for each sample. Experiments were performed at room temperature, but neither temperature nor $\mathrm{PH}$ were monitored.

\subsubsection{Establishing the kinetics of removal}

The kinetics of removal were analyzed using a $0.5 \mathrm{ppm}$ mixture of E2, EE2, E1, BPA, and DES following the same procedure as that outlined above for treatment of water with a mixture of EDCs. NIP concentrations of 50 and $500 \mathrm{mg} / \mathrm{L}$ were tested for contact times of 10 minutes, 30 minutes, 1 hour, 2 hours, 5 hours, 1 day, and 4 days. Experiments were performed at room temperature, but neither temperature nor $\mathrm{PH}$ were monitored.

\subsubsection{Removal of pharmaceuticals}

Tests were conducted with 12 pharmaceuticals: acetaminophen, caffeine, carbamazepine, cloxacillin, diphenhydramine, enrofloxacin, lincomycin, oxacillin, sulfadiazine, sulfamethizole, sulfanilamide, and sulfathiazole in deionized water and wastewater. Secondary wastewater effluent samples were collected from the Robert O. Pickard Environmental Centre in Ottawa, Ontario. The Robert O. Pickard Environmental Centre was chosen for wastewater samples because it treats a combination of domestic, commercial and industrial wastewater with conventional wastewater treatment methods including a primary clarifier and activated sludge treatment (City of Ottawa, 2012). Refer 
to Appendix B for a summary of the wastewater characteristics. Surface water samples were not tested due to limits in the number of samples that were feasible. $200 \mathrm{ng} / \mathrm{L}$ of acetaminophen, $50 \mathrm{ng} / \mathrm{L}$ of sulfathiazole, and $100 \mathrm{ng} / \mathrm{L}$ of the remaining pharmaceuticals were spiked into $2.5 \mathrm{~L}$ each of deionized water and wastewater. A $100 \mathrm{~g} / \mathrm{L}$ solution of NIP was created and sonicated to disperse the NIP. Following sonification there was no visible settling of the particles. $1 \mathrm{~L}$ of each of the spiked solutions was treated by adding $10 \mathrm{~mL}$ of the concentrated NIP solution to give a final concentration of $1 \mathrm{~g} \mathrm{NIP/L}$. The samples were mixed for 24 hours on a shaker table before being centrifuged at 10, $000 \mathrm{rpm}$ for 1 hour to remove the NIP. The centrate was poured into sample bottles, which were packed in a cooler and shipped to Axys Analytical Services (Sidney, BC). Experiments were performed at room temperature, but neither temperature nor PH were monitored.

\subsection{RESULTS AND DISCUSSION}

\subsubsection{Identification of important parameters}

Because adsorption can be affected by a wide range of factors, tests were completed to evaluate which variables were important prior to proceeding with further experiments. The effects of binding time, E2 concentration, particle mass and NIP batch were investigated for adsorption of E2 onto NIP. No effects were observed for particle mass $(5,10,15$, or 20 $\mathrm{mg} / \mathrm{L})$ or binding time $(5,7.5$, or 10 minutes $)$, but there was a significant effect of NIP batch (1 or 2$)$ and initial concentration $(2,6,10$, or $14 \mathrm{ppb}$ of E2). Linear regression was used to determine the effects of each parameter on the amount of E2 adsorbed; 95\% confidence intervals for the coefficients were 0.000359 to 0.000117 for concentration and -0.00235 to -0.00259 for batch. It is not surprising that there was significant batch-to-batch 
variation, because the NIP particles were prepared in small batches in the lab and experimental error and small differences in measured quantities could have had a significant effect on the final NIP produced. Because there was significant batch-to-batch variation in removal efficiencies, several batches were prepared, mixed well and used for the rest of the experiments. Refer to Appendix E for detailed results of this investigation.

The adsorption efficiency was greater than $95 \%$ for all NIP concentrations $(5-20 \mathrm{mg} / \mathrm{L})$ tested, and the results showed a high degree of removal for the E2. It is also possible that the contact time and NIP concentration had little effect on performance because of the relatively high particle concentration.

\subsubsection{Treatment of water with a single EDC}

The advantage of using NIP over MIP for water and wastewater treatment is that NIP are non-specific and should be able to remove many different water contaminants. The results from the previous experiments for E2 can be compared to experiments performed under similar conditions for other EDCs. Due to their specificity, MIP designed with an E2 template would be expected to show a very high removal for E2, and lower removal for other EDCs. The template-less NIP; however, should be able to remove all the EDCs through non-specific interactions. To test this, adsorption of E2 was compared to adsorption of $17 \alpha$-ethinylestradiol (EE2), bisphenol A (BPA), and diethylstilbestrol (DES). Removal of each of these EDCs was evaluated by preparing standard solutions of varying concentrations and mixing them with $5 \mathrm{mg} / \mathrm{L}$ of NIP in $1.5 \mathrm{~mL}$ centrifuge tubes 
for 5 minutes. Therefore, results can be compared with those previously obtained for E2 for $5 \mathrm{mg} / \mathrm{L}$ of NIP and a contact time of 5 minutes.

Figure 5-1 shows the removal of E2, EE2, and BPA with NIP. Error bars, representing the standard deviation are shown. For E2, the error bars represent the pooled standard deviations, and were very small and not visible. For E2, the NIP exhibited a high binding efficiency greater than $98 \%$ for all of the concentrations studied. The high removal efficiency is also related to the NIP concentration used, and can be lower at lower NIP concentrations. The results show that NIP have an excellent ability to remove E2 from water within a very short contact time. 


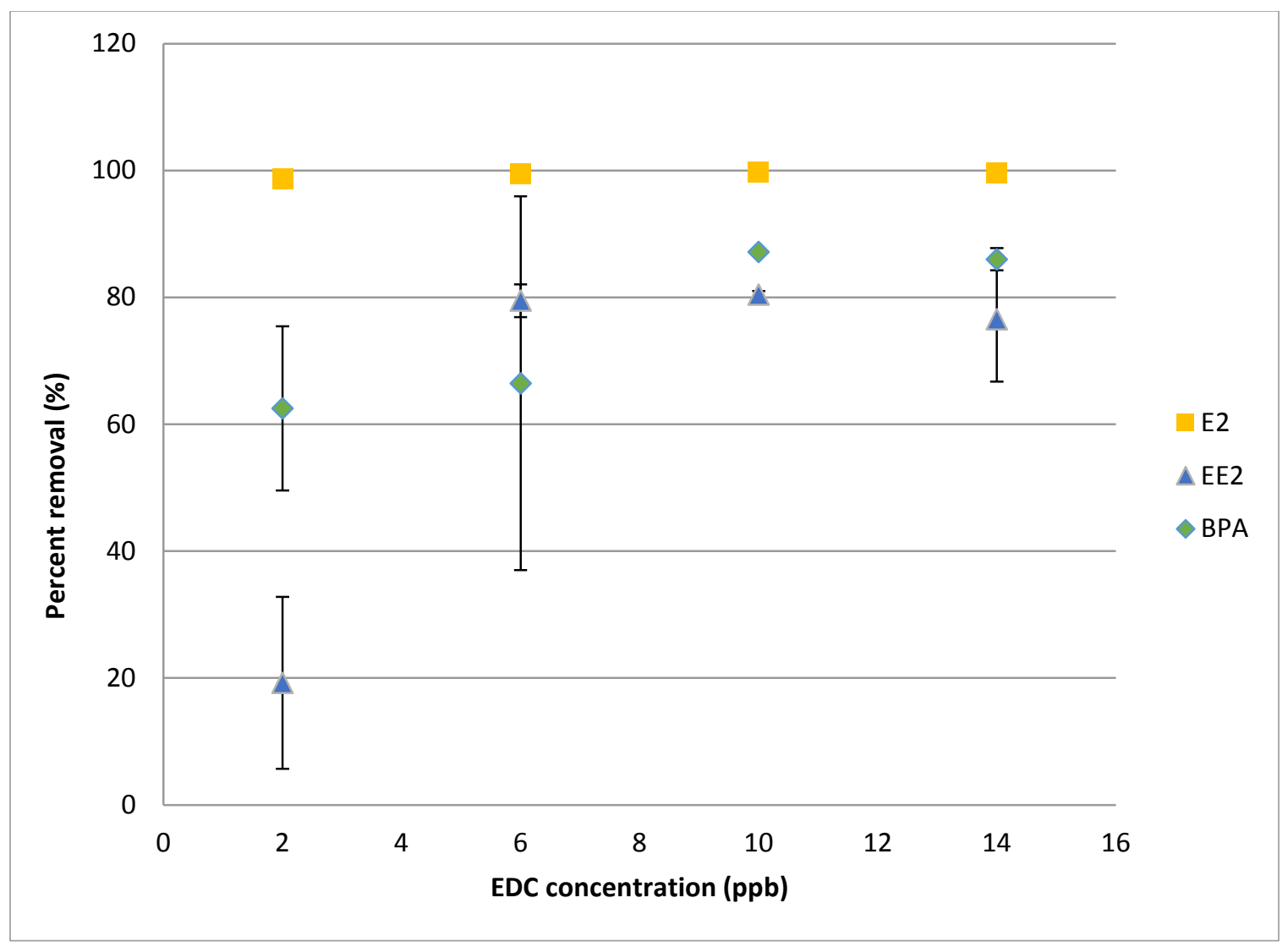

Figure5-1: Removal of E2, EE2, and BPA (2-14 ppb) with NIP

For EE2, Figure 5-1 shows an increase in efficiency with increasing E2 concentration, up to $80 \pm 0.6 \%$ removal for an initial E2 concentration of $6 \mathrm{ppb}$ (from $19 \pm 14 \%$ at $2 \mathrm{ppb}$ ), followed by an area of uniform removal (80\%) with increasing concentration. At 2 ppb E2, $19 \%$ removal was measured but the data point also had larger error bars compared to the other points. This was a very low initial concentration, and the concentration of the final sample, measured after some removal, was approaching the detection limit of the analysis. Further optimization of the analytical method may be required to reduce the detection limit. 
For BPA, the percent removal increased from $62 \pm 13 \%$ for $2 \mathrm{ppb}$ to $87 \pm 0.6 \%$ for $10 \mathrm{ppm}$ before leveling off. Again, the lower removal efficiencies for very low concentrations may have been due to the short contact time. Larger uncertainty for very low concentrations also occurred due to difficulties measuring concentrations close to the detection limit.

Figure 5-2 shows the percent removal for DES with $5 \mathrm{mg} / \mathrm{mL}$ of NIP for a contact time of 5 minutes. For DES, the initial concentrations tested were in the range of 5-20 ppm instead of 2-14 ppb because DES had a much higher detection limit. The percent removal for DES decreased through this range from $97 \pm 2 \%$ for $5 \mathrm{ppm}$ to $78 \pm 0.75 \%$ for $20 \mathrm{ppm}$. The decrease in efficiency for high DES concentrations likely occurred because the NIP were approaching saturation, but the quantity of DES adsorbed/mass of NIP increased from 15 ppm $(2.4 \pm 0.2 \mathrm{mg} / \mathrm{g})$ to $20 \mathrm{ppm}(3.1 \pm 0.03 \mathrm{mg} / \mathrm{g})$, indicating that the NIP had not reached saturation yet. Overall, these results demonstrate excellent potential for use of NIP to remove DES, because the concentrations tested were more than a thousand times those tested for E2, EE2, and BPA; yet, up to 97\% removal was achieved. 


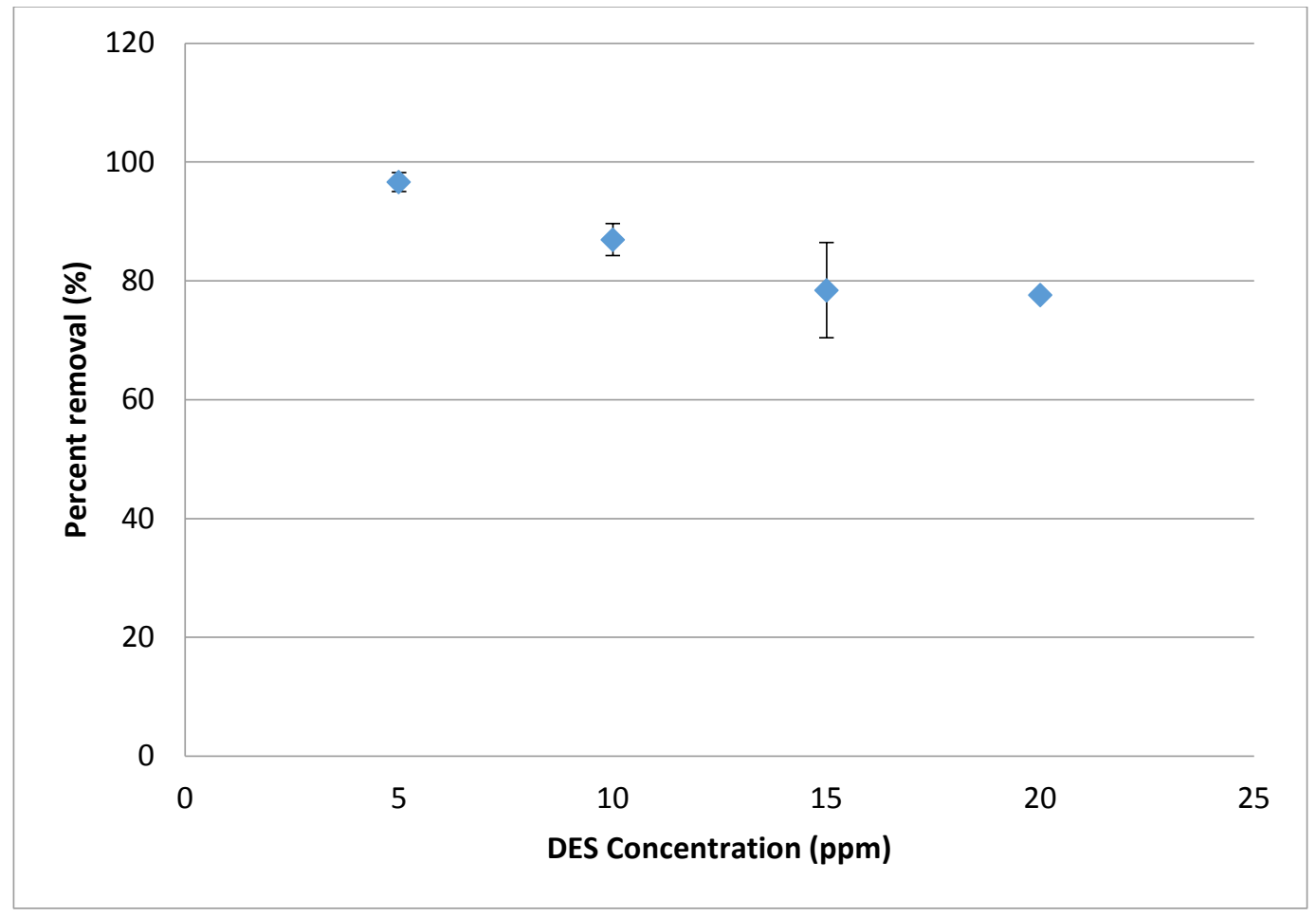

Figure 5-2: Removal of DES (5-20 ppm) with NIP

For 2-14 ppb of E2, EE2, and BPA, the maximum percent removals achieved after 5 minutes were $98 \%, 80 \%$, and $87 \%$, respectively. Therefore, the NIP did show a preference for E2 followed by BPA, and then EE2. For NIP, the mechanism for adsorption is nonspecific and occurs through Van der Waals forces and hydrogen bonds (Pichon and Chapuis-Hugon, 2008). The order of preference is expected to be inversely related to the hydrophobicity of the molecules, leading to an order of E2>EE2>BPA (NCBI, 5757; NCBI, 5991; NCBI 6623). This accurately predicts the preference for E2 over EE2 and BPA, but not for BPA over EE2. However, it is important to note that the contact time was relatively short and the order of removal may change with increased contact time. Generally, larger molecular size molecules have lower solubilities, but also diffuse more 
slowly in water, so adsorbates with lower solubilities may have adsorbed more slowly. The molecular weights of E2, EE2 and BPA follow the order: EE2 $>$ E2 $>$ BPA, which may explain why the smaller BPA molecule was adsorbed to a greater extent after a short contact time. In general, the NIP showed an excellent potential for removal of E2, EE2, BPA, and DES,

\subsubsection{Treatment of water with a mixture of EDCs}

NIP are best suited for the removal of many EDCs simultaneously from a water or wastewater stream. Additional tests were completed with a mixture of E2, EE2, estrone (E1), BPA, and DES to determine the removal efficiencies in the presence of competing compounds and compare them to the removal efficiencies measured in the previous section when there was no competing compound.

The capacity of the NIP was then tested over a wide range of concentrations to determine the working range for the NIP. For this set of tests, $50 \mathrm{~mL}$ sample sizes were used to further dilute the NIP, and NIP concentrations were varied from $0.1-1000 \mathrm{mg} / \mathrm{L}$ over several orders

of magnitude. As seen in Figure 5-2 for DES, a DES concentration more than a thousand times higher than the concentrations tested for E2, EE2, and BPA, exhibited a very high removal (97\%), and the NIP appear to work effectively over a wide range of concentrations. A mixture of the five EDCs was prepared using $0.5 \mathrm{ppm}$ of each, and the contact time was 24 hours. Samples were analyzed with LC-MS-MS to clearly distinguish between different EDCs in the mixture. 
Figure 5-3 shows the results obtained, with percent removal on the y-axis, and NIP concentration on the $\mathrm{x}$-axis. The results provided are averages of 3 experimental replicates, each of which represents the average of 6 analytical replicates. The error bars represent the standard deviations of the experimental replicates. Referring to Figure 5-3, zero percent removal was within 1 standard deviation of the mean percent removal for all 5 EDCs for NIP concentrations up to $100 \mathrm{mg} / \mathrm{L}$, indicating negligible removal. For EE2, addition of $100 \mathrm{mg} / \mathrm{L}$ of NIP also led to negligible removal. Percent removal values for E1, E2, BPA, and DES were low for $100 \mathrm{mg} / \mathrm{L}$ of NIP and ranged from $10 \pm 3 \%$ for DES to $13 \pm 1 \%$ for E1. For $1000 \mathrm{mg} / \mathrm{L}$ of NIP, $30 \pm 12 \%$ of BPA, $33 \pm 8 \%$ of E2, $24 \pm 13 \%$ of EE2, $49 \pm 6 \%$ of DES, and $35 \pm 8 \%$ of E2 were removed from a $0.5 \mathrm{ppm}$ mixture. Comparison between the results shown in Figure 5-3, below and those shown in Section 5.4.3 was not completed because the range of concentrations of both the adsorbates and the NIP were different. The ratio of DES to NIP was similar for both experiments, but, as seen in Section 5.4.1, the effects of varying EDC concentration and varying NIP concentration were not the same in all cases, therefore it is not possible to make meaningful comparisons between the two sets of experiments. 


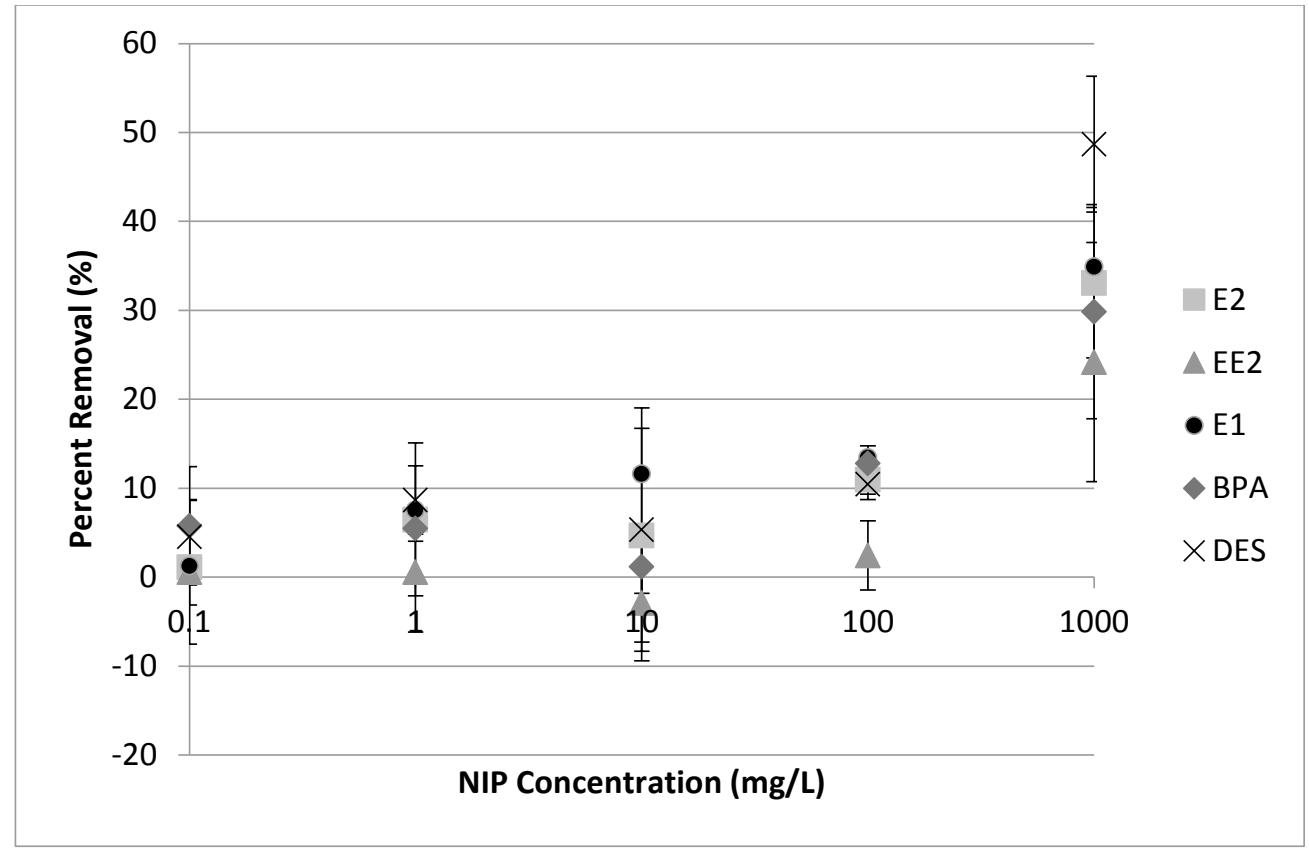

Figure 5-3: Treatment of a mixture of EDCs with NIP

Adsorption of hydrophobic EDCs onto NIP is typically governed by the hydrophobicity of each EDC (Zhang and Hu, 2012). For a NIP concentration of $1000 \mathrm{mg} / \mathrm{L}$, the order of preference was: $\mathrm{DES}>\mathrm{E} 1>\mathrm{E} 2>\mathrm{BPA}>\mathrm{EE} 2$. This was roughly the order of the hydrophobicity of the molecules which is: DES $>$ E2 $>$ EE2 $>$ BPA $>$ E1 (NCBI, 448537; NCBI, 5757; NCBI, 5991; NCBI, 6623; NCBI, 5870). E2 is more hydrophobic than E1 and EE2 is more hydrophobic than BPA, but it is important to note that the percent removal values for BPA and EE2 were within 1 standard deviation of each other. Overall, the ability of NIP to remove a range of EDCs from water was good. Further testing should be completed with higher NIP to EDC ratios to achieve the near-complete removal seen in Figures 5-1 and 5-2, and to determine the optimum NIP concentration. 


\subsubsection{Establishing the kinetics of removal}

In the determination of the important parameters for removal of E2 with NIP, contact time was not identified as an important controlling factor for removal. However, the concentration of NIP used for those tests was later found to be very high $(\mathrm{mg} / \mathrm{mL}$ versus $\mathrm{mg} / \mathrm{L}$ ), and this may have had an effect on the results. For this reason, removal kinetics were studied for the $0.5 \mathrm{ppm}$ mixture of E2, EE2, E1, BPA, and DES in deionized water over a four-day period.

A standard solution with $0.5 \mathrm{ppm}$ each of E2, EE2, E1, BPA, and DES was treated with either 50 or $500 \mathrm{mg} / \mathrm{L}$ of NIP. Samples were taken after 10 minutes, 30 minutes, 1 hours, 2 hours, 5 hours, 1 day, and 4 days. The results, shown in Figures 5-4 (a) and (b), are averages of three experimental replicates, each of which is the average of six analytical replicates. 


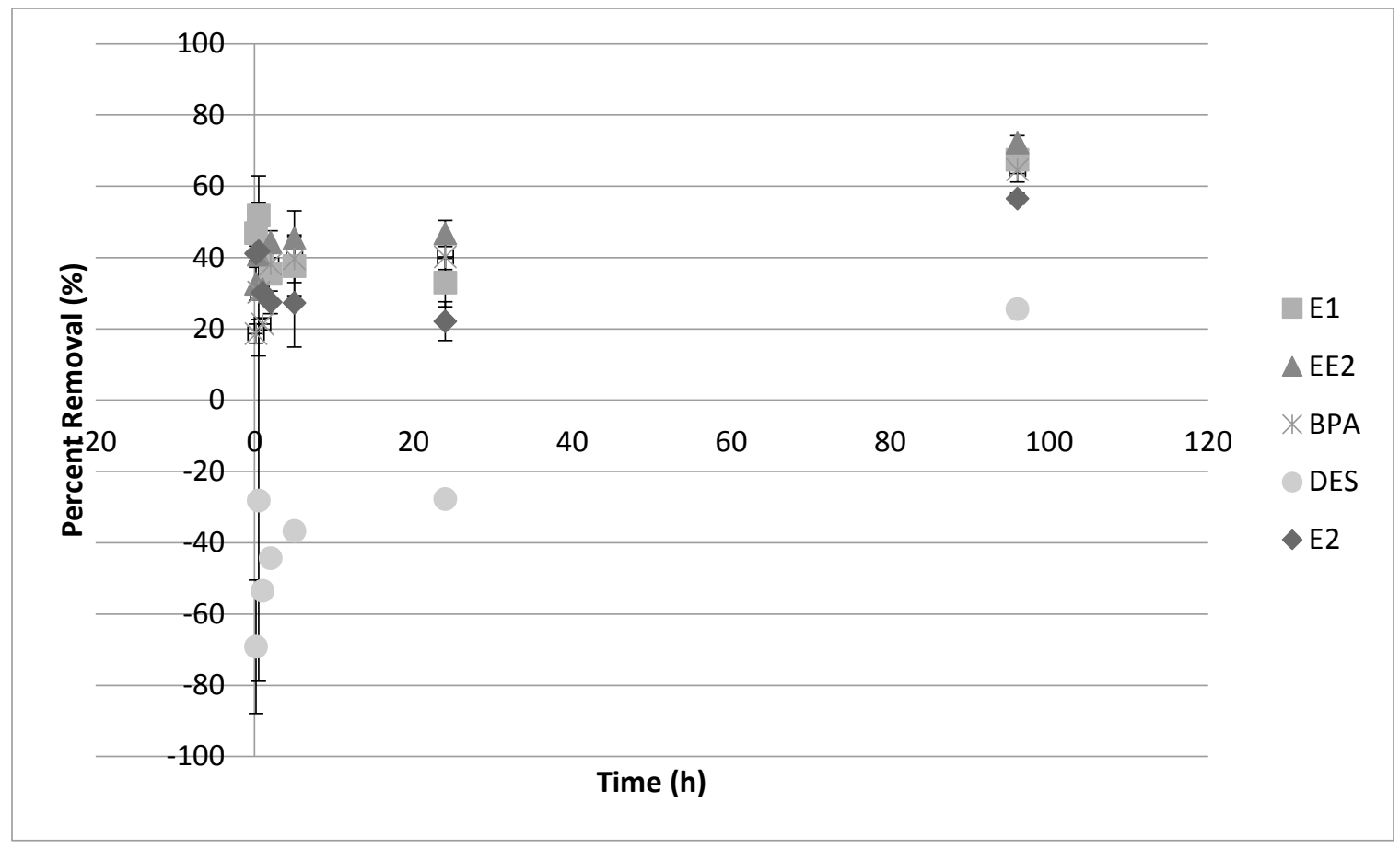

Figure 5-4 (a): Kinetics of removal for a mixture of 5 EDCs with $50 \mathrm{mg} / \mathrm{L}$ of NIP over a 4 day period

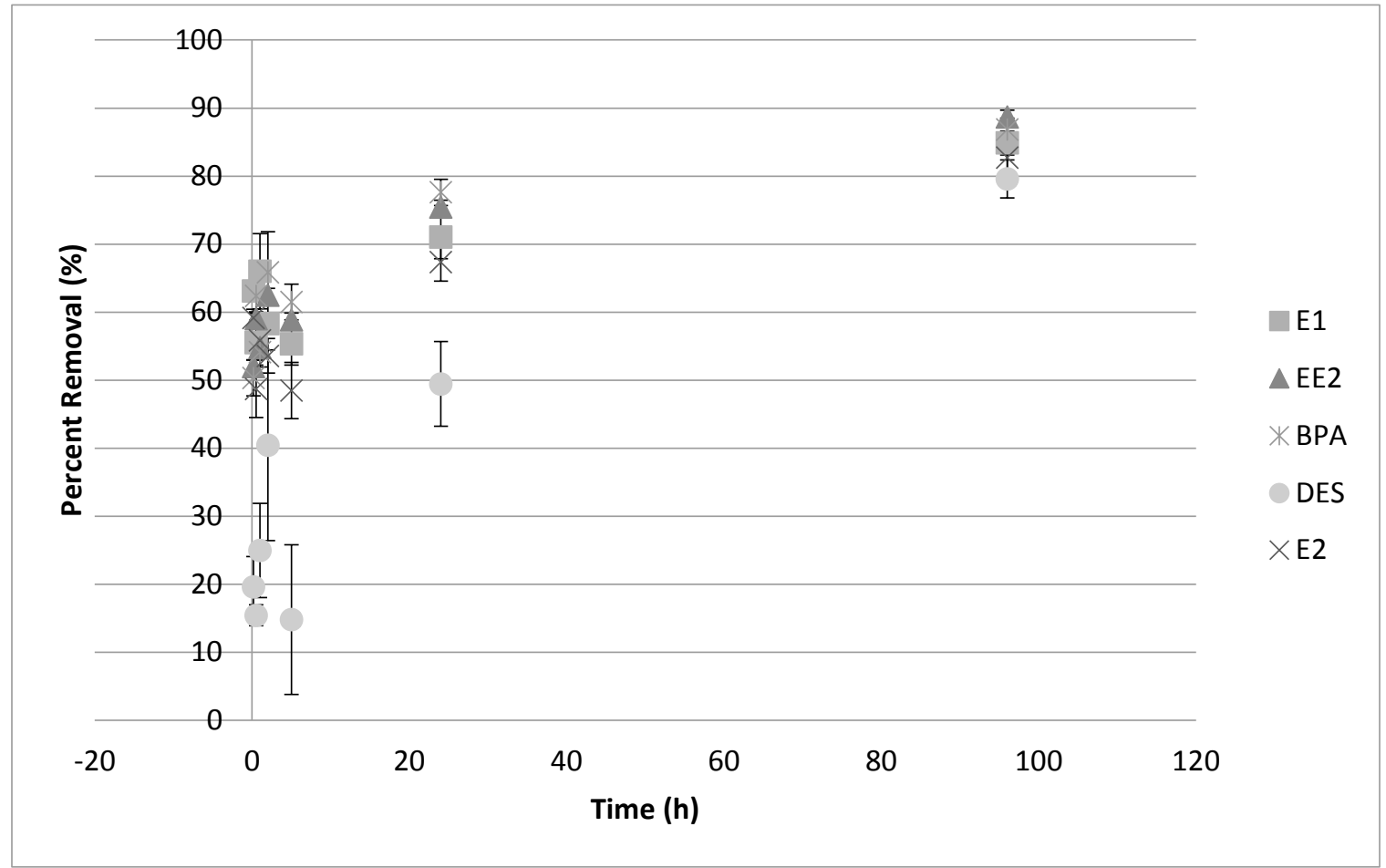


Figure 5-4 (b): Kinetics of removal for a mixture of 5 EDCs with $500 \mathrm{mg} / \mathrm{L}$ of NIP over a 4 day period

Figure 5-4 (a) shows the adsorption of E2, EE2, E1, BPA, and DES onto $50 \mathrm{mg} / \mathrm{L}$ NIP over a 4 day contact time. Percent removal values were used to describe adsorption rather than loadings to highlight the fact that the experiments were not conducted at equilibrium, and the figures are not isotherms. For adsorption of E2 onto $50 \mathrm{mg} / \mathrm{L}$ of NIP, there were some initial fluctuations in the percent removal. After 10 minutes, $41 \pm 2 \%$ of the E2 had been removed, but this decreased to $22 \pm 5 \%$ after 1 day, before increasing to $57 \pm 2 \%$ after 4 days. These fluctuations may have been caused by competitive NOM and heavy metal complexation, adsorption to the bottle, or competition with other EDCs for adsorption sites.

For adsorption of EE2, the percent removal generally increased with increasing contact time, although a dip in the percent removal was observed after 1 hour. Overall, the removal of EE2 increased from $33 \pm 0.5 \%$ after 10 minutes to $72 \pm 2 \%$ after 4 days. It is interesting to compare these results with those for E2. After 10 minutes, the percent removal was higher for E2 ( $41 \pm 2 \%$ for $\mathrm{E} 2$ vs. $33 \pm 5 \%$ for EE2), but after 2 days, the percent removal was higher for EE2 $(72 \pm 2 \%$ for EE2 vs. $57 \pm 2 \%$ for E2). This indicates that although the removal kinetics may have been faster for E2 (which was suspected based on the results in Figure 5-1), the final amount adsorbed was higher for EE2.

For E1, Figure 5-4 (a) shows that the amount adsorbed with $50 \mathrm{mg} / \mathrm{L}$ of NIP initially decreased from $47 \pm 2 \%$ after 10 minutes to $33 \pm 7 \%$ after 1 day, before increasing to a final percent removal of $67 \pm 4 \%$ after 4 days. For E1, the amount adsorbed after 10 
minutes, was higher than that for E2 and EE2. However, after 10 days, the percent removal was higher for EE2. This can be explained by looking at the molecular weights of E1, E2, and EE2, which are 270, 272, and $296 \mathrm{~g} /$ mole, respectively. Smaller molecules can be expected to diffuse more quickly through solution, and adsorb more quickly (Crittenden et al., 2005). Therefore the kinetics of adsorption for E1 can be expected to be faster than that for EE2.

For BPA, the amount adsorbed increased from $19 \pm 3 \%$ after 10 minutes to $65 \pm 3 \%$ after 4 days for $50 \mathrm{mg} / \mathrm{L}$ of NIP, as shown in Figure $5-4$ (a). For $500 \mathrm{mg} / \mathrm{L}$ of NIP, the percent removal fluctuated, but generally increased from $63 \pm 1 \%$ after 10 minutes to $85 \pm 2 \%$ after 4 days. After 10 minutes the percent removal of BPA was lower than that for E2, EE2, and E1. After 4 days, it was higher than E2 for both E2 and E1. This was unexpected, because the molecular weight of BPA was $228 \mathrm{~g} / \mathrm{mole}$, which was the lowest of the 5 EDCs studied, so BPA was expected to have the fastest adsorption kinetics.

For DES with $50 \mathrm{mg} / \mathrm{L}$ of NIP, negative percent removal values were recorded for sample times less than 4 days. This was likely due to a source of DES contamination or due to the concentration of the controls used in the calculations. The concentrations of the controls were not measured for each sampling time and were only measured once after 4 days. If a significant portion of the DES was removed by other mechanisms between 1 and 4 days, this could lead to falsely low or negative percent removal values. After 4 days, $26 \pm 6 \%$ of the DES was removed, which was lower than all the other EDCs tested. 
Figure 5-4 (b) shows the adsorption of E2, EE2, E1, BPA, and DES onto $500 \mathrm{mg} / \mathrm{L}$ NIP over a 4 day contact time. For E2 with $500 \mathrm{mg} / \mathrm{L}$ of NIP, the percent removal fluctuated during the first 24 hours, before increasing from $67 \pm 3 \%$ to $83 \pm 25 \%$ between 1 and 4 days.

For adsorption of EE2 with $500 \mathrm{mg} / \mathrm{L}$ of NIP, similar trends to those shown in Figure 5-4 (a) were seen. The amount of EE2 adsorbed increased from $52 \pm 2 \%$ after 10 minutes to 84 $\pm 2 \%$ after 4 days. Also, similar to the $50 \mathrm{mg} / \mathrm{L}$ NIP results, the amount adsorbed after 10 minutes was higher for E2 (59 $\pm 1 \%$ for E2 vs. $52 \pm 2 \%$ for EE2), but after 4 days, more EE2 was adsorbed $(89 \pm 2 \%$ for EE2 vs. $83 \pm 2 \%$ for E2). The magnitude of the difference was less than that for $50 \mathrm{mg} / \mathrm{L}$ of NIP; however, probably because there was less competition for adsorption sites due to a greater availability of NIP.

For adsorption of E1 with $500 \mathrm{mg} / \mathrm{L}$ of NIP shown in Figure 5-4 (b), the amount adsorbed fluctuated before increasing from $71 \pm 3 \%$ after 1 day to $85 \pm 2 \%$ after 4 days. Similar to the results shown in Figure 5-4 (a), the amount adsorbed after 10 minutes, was higher than that for E2 and EE2. However, after 10 days, the percent removal was higher for EE2. This can be explained by the molecular weight of EE2, which is larger than that of E1 and E2, decreasing the rate of adsorption.

For adsorption of BPA with $500 \mathrm{mg} / \mathrm{L}$ of NIP, the percent removal fluctuated, but generally increased from $63 \pm 1 \%$ after 10 minutes to $85 \pm 2 \%$ after 4 days. After 10 minutes, for both 50 and $500 \mathrm{mg} / \mathrm{L}$ of NIP, the percent removal of BPA was lower than that for E2, 
EE2, and E1. After 4 days, it was higher than E2 for both $50 \mathrm{mg} / \mathrm{L}$ and both E2 and E1 for $500 \mathrm{mg} / \mathrm{L}$, although the magnitude of the difference was lower for $500 \mathrm{mg} / \mathrm{L}$ due to reduced competition.

For adsorption of DES with $500 \mathrm{mg} / \mathrm{L}$ of NIP, the amount removed with time fluctuated, but generally increased from $20 \pm 4 \%$ after 10 minutes to $80 \pm 3 \%$ after 4 days, which, again, was lower than the removal of any other EDCs.

For a mixture of E2, EE2, E1, BPA, and DES in water at $0.5 \mathrm{ppm}$, equilibrium was not reached after 4 days. However, high removals of $80 \%$ or greater were achieved for all five of these EDCs with $500 \mathrm{mg} / \mathrm{L}$ of NIP.

\subsubsection{Removal of pharmaceuticals}

To further test the potential for NIP to treat a wide variety of micropollutants, the NIP were tested for their ability to remove a range of pharmaceuticals from both deionized water and wastewater. Acetaminophen, caffeine, carbamazepine, cloxacillin, diphenhydramine, enrofloxacin, lincomycin, oxacillin, sulfadiazine, sulfamethizole, sulfanilamide, and sulfathiazole were all spiked into both deionized water and wastewater in concentrations ranging from 50-200 $\mathrm{ng} / \mathrm{L}$. An NIP concentration of $1 \mathrm{~g} / \mathrm{L}$ was used to treat the pharmaceuticals over a 24 hour contact time.

Figure 5-5 provides the results for treatment of the pharmaceuticals in deionized water. The spiked concentration, untreated concentration, and treated concentrations are shown 
for each of the 12 pharmaceuticals. The untreated samples were handled using exactly the same procedure as the treated samples, but NIP were not added. This was done to account for any effects of NOM and heavy metal complexation, adsorption to the bottle, dilution errors, or contamination. The pharmaceuticals were tested at very low concentrations, and avoiding errors at these concentrations can be difficult. Looking at Figure 5-5, many of the concentrations measured for the untreated samples were close to the spiked concentrations, except those for acetaminophen and caffeine. However, since the interest here is not the actual concentrations, but rather the removal with NIP, this is not an issue. Comparing the treated and untreated samples (before and after), some of the pharmaceuticals (carbamazepine, cloxacillin, diphenhydramine, enrofloxacin, and oxacillin) exhibited a high $(>50 \%)$ degree of removal; some (caffeine, sulfadiazine, sulfamethizole, and sulfathiazole) were removed to some degree $(<50 \%)$; and for a few (acetaminophen, lincomycin, and sulfanilamide), the treated concentrations were higher than the untreated concentrations. Treated concentrations that were higher than untreated concentrations were likely due to differences measuring the pharmaceuticals at very low concentrations. These preliminary results show some promise for the NIP: 5 out of the 12 pharmaceuticals were removed by more than $50 \%$. 


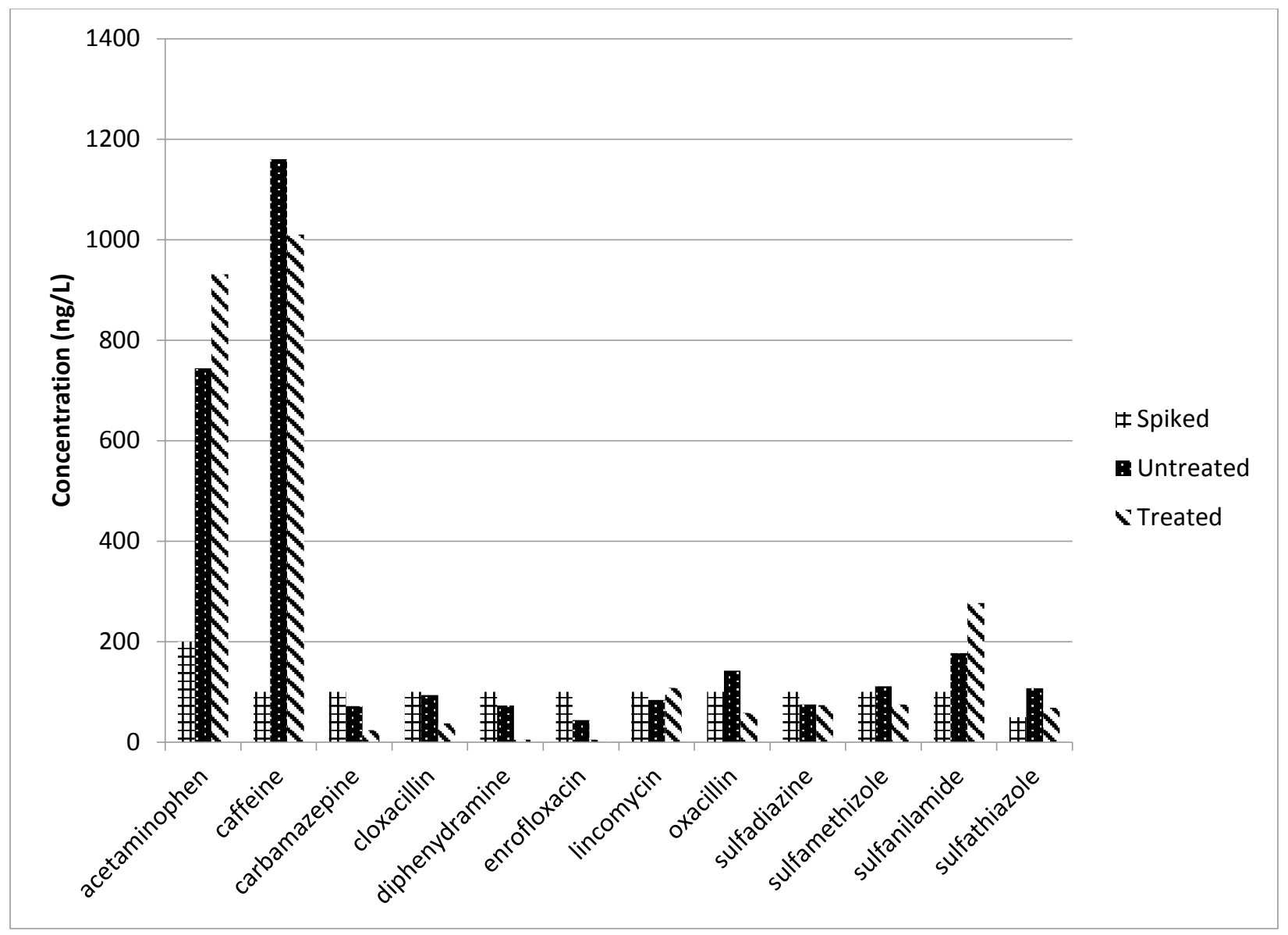

Figure 5-5: Treatment of pharmaceuticals in deionized water with NIP

The same 12 pharmaceuticals were also spiked into a sample of secondary wastewater effluent collected from the Robert O. Pickard Environmental Centre in Ottawa, Ontario. However, due to difficulties anticipating the final concentrations of the pharmaceuticals after spiking, carbamazepine, sulfadiazine, sulfamethizole, sulfanilamide, and sulfathiazole could not be measured by the lab because they were outside of the lab's linear calibration range. Acetaminophen, caffeine, cloxacillin, diphenhydramine, enrofloxacin, lincomycin, and oxacillin were measured. Figure 5-6 shows the spiked, untreated, and treated concentrations for these 7 pharmaceuticals. For the wastewater sample, with the 
exception of acetaminophen, all of the concentrations measured in the untreated samples were higher than the spiked concentrations. This was not surprising because the wastewater itself may have contained low concentrations of these pharmaceuticals.

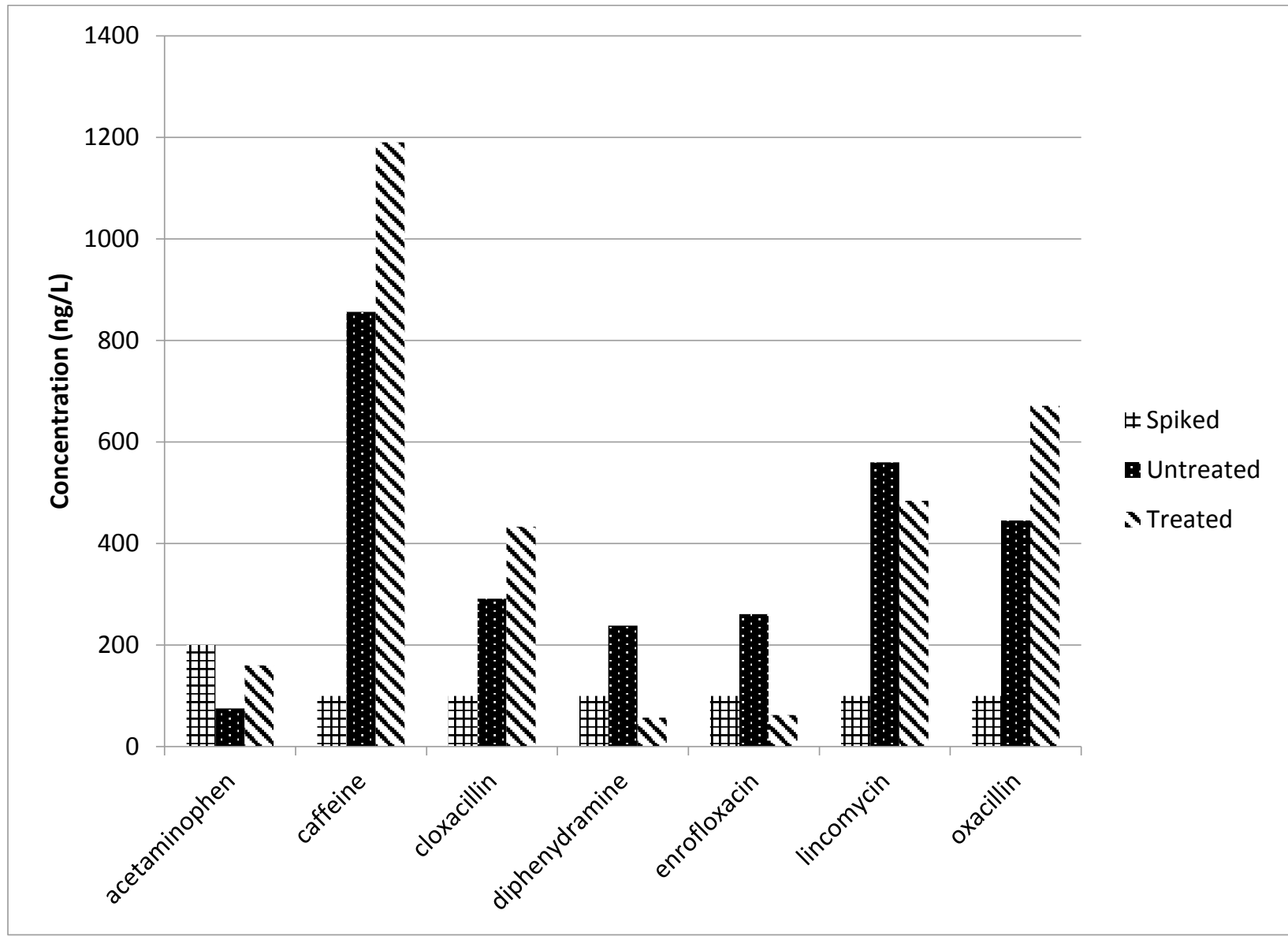

Figure 5-6: Treatment of pharmaceuticals in wastewater with NIP

The wastewater results were less promising than those for deionized water. The presence of competing substances in the wastewater may have decreased the removal of the pharmaceuticals and a higher concentration of NIP may be required to treat wastewater. For the wastewater, diphenhydramine and enrofloxacin exhibited high (>50\%) removals, 
lincomycin was removed to some degree $(<50 \%)$, and acetaminophen, caffeine cloxacillin, and oxacillin had higher treated than untreated concentrations. Again, difficulties measuring pharmaceuticals at very low concentrations and likely variations in results makes it difficult to draw any firm conclusions, but the results do show some potential, and further investigation is merited.

In addition to the 12 spiked pharmaceuticals, measurements were also taken for 32 other pharmaceuticals in both the deionized water and wastewater samples. Many of these were not detected, but some did show some degree of removal. The concentrations measured are presented in Appendix F.

\subsection{CONCLUSIONS}

Preliminary tests demonstrated the potential for NIP to treat a wide variety of EDCs and pharmaceuticals. For water spiked with a single EDC, NIP were able to remove $98 \%$ of spiked E2, $80 \%$ of EE2, $87 \%$ of BPA, and up to $97 \%$ of DES. It was also investigated whether or not the NIP could effectively treat water spiked with a mixture of different micropollutants. In a mixed solution with $0.5 \mathrm{ppm}$ each of E2, EE2, E1, BPA, and DES, percent removals with $500 \mathrm{mg} / \mathrm{L}$ of NIP were in the range of $24 \pm 13 \%$ (E2) to $49 \pm 6 \%$ (DES), and increased with increasing NIP concentrations, suggesting the potential for more removal with higher particle concentrations. Further testing with a complex mixture of 12 pharmaceuticals in both deionized water and wastewater were less conclusive likely due to very low concentrations and variability in sample processing and measurements. Nonetheless, NIP were able to remove 9 out of 12 of the pharmaceuticals to some degree 
from deionized water and 3 out of 7 of the pharmaceuticals measured in wastewater. These tests are not conclusive, but do indicate that NIP merits further study as a potential treatment method for micropollutants in water and wastewater.

\subsection{REFERENCES}

Anderson, P., Denslow, N., Drewes, J.E., Olivieri, A., Schlenk, D., Snyder, S., 2010. Final Report ( Draft for Public Comments ) Monitoring Strategies for Chemicals of Emerging Recommendations of a Science Advisory Panel Panel Members. Sacramento California.

Certificate of accreditation - Canadian Association for Laboratory Accreditation. 2010.

City of Ottawa,. Regulatory Compliance Report: Wastewater And Stormwater Systems 2012 Annual Summary Report. Ottawa: City of Ottawa, 2013.

Alexander, C., 2006. Molecular Imprinting Science and Technology: a Survey of the Literature for the Years up to and Including 2003. Journal of Molecular Recognition. 19, 106-80.

Dai, C.-M., J. Zhang, Y.-L. Zhang, X.-F. Zhou, Y.-P. Duan, and S.-G. Liu. 2012 Selective Removal of Acidic Pharmaceuticals from Contaminated Lake Water Using Multi-Templates Molecularly Imprinted Polymer. Chemical Engineering Journal, 211-212 302-309.

Deng, S., D. Shuai, Q. Yu, J. Huang, and G. Yu. 2009 Selective Sorption of Perfluorooctane Sulfonate on Molecularly Imprinted Polymer Adsorbents. Frontiers of Environmental Science \& Engineering in China, 3 (2), 171-177. 
Englert, B. (US E. 2007 Method 1694 : Pharmaceuticals and Personal Care Products in Water, Soil, Sediment, and Biosolids by HPLC / MS / MS. USEPA 821-R-08002,. Washington, D.C.

Evgenidou, E. N., I. K. Konstantinou, and D. a. Lambropoulou. 2015 Occurrence and Removal of Transformation Products of PPCPs and Illicit Drugs in Wastewaters: A Review. Science of The Total Environment, 505 905-926.

Gillesby, B. E., and T. R. Zacharewski. 1998 Exoestrogens: Mechanisms of Action and Strategies for Identification and Assessment. Environmental Toxicology and Chemistry, 17 (1), 3-14.

Kuzmanovic', M., A. Ginebreda, M. Petrovic', and D. Barcelo'. 2014 Risk Assessment Based Prioritization of 200 Organic Micropollutants in 4 Iberian Rivers. Science of the Total Environment, 504 289-299.

Masqué, N. 2001 Molecularly Imprinted Polymers: New Tailor-Made Materials for Selective Solid-Phase Extraction. TrAC Trends in Analytical Chemistry, 20 (9), $477-486$.

Meng, Z., Q. Zhang, M. Xue, D. Wang, and A. Wang. 2012 Removal of 2,4,6Trinitrotoluene from "Pink Water" Using Molecularly-Imprinted Absorbent. Propellants, Explosives, Pyrotechnics, 37 (1), 100-106.

Murray, A., and B. Ormeci. 2012 Application of Molecularly Imprinted and Non-Imprinted Polymers for Removal of Emerging Contaminants in Water and Wastewater Treatment: a Review. Environmental Science and Pollution Research, 19 (9), 3820-30. 
National Center for Biotechnology Information. PubChem Compound Database; $\mathrm{CID}=5757, \quad \mathrm{https}: / /$ pubchem.ncbi.nlm.nih.gov/compound/5757 (accessed September 10, 2015).

National Center for Biotechnology Information. PubChem Compound Database; $\mathrm{CID}=5870, \quad$ https://pubchem.ncbi.nlm.nih.gov/compound $/ 5870 \quad$ (accessed September 10, 2015).

National Center for Biotechnology Information. PubChem Compound Database; CID=5991, https://pubchem.ncbi.nlm.nih.gov/compound/5991 (accessed September 10, 2015).

National Center for Biotechnology Information. PubChem Compound Database; $\mathrm{CID}=6623, \quad \mathrm{https}: / /$ pubchem.ncbi.nlm.nih.gov/compound/6623 (accessed September 10, 2015).

National Center for Biotechnology Information. PubChem Compound Database; $\mathrm{CID}=448537, \quad$ https://pubchem.ncbi.nlm.nih.gov/compound/448537 (accessed September 10, 2015).

Pichon, V., and F. Chapuis-Hugon. 2008 Role of Molecularly Imprinted Polymers for Selective Determination of Environmental Pollutants--a Review. Analytica Chimica Acta, 622 (1-2), 48-61.

Venkatesh, A., N. Chopra, and R. J. Krupadam. 2014 Removal of Acutely Hazardous Pharmaceuticals from Water Using Multi-Template Imprinted Polymer Adsorbent. Environmental Science and Pollution Research International, 21 (10), 6603-11. 
Yang, M., M. S. Park, and H. S. Lee. 2006 Endocrine Disrupting Chemicals: Human Exposure and Health Risks. Journal of Environmental Science and Health. Part C, Environmental Carcinogenesis \& Ecotoxicology Reviews, 24 (2), 183-224.

Zhang, Z. B., and J. Y. Hu. 2012 A Quantitative Method Evaluating the Selective Adsorption of Molecularly Imprinted Polymer. Journal of Environmental Science and Health. Part A, Toxic/Hazardous Substances \& Environmental Engineering, 47 (6), 837-42. 


\title{
CHAPTER 6: USE OF NON-IMPRINTED POLYMERS FOR REMOVAL OF HEAVY METAL IONS FROM WATER AND WASTEWATER
}

\author{
Audrey Murray and Banu Örmeci \\ Department of Civil and Environmental Engineering, Carleton University, 1125 Colonel \\ By Drive, Ottawa ON K1S 5B6, Canada.
}

\subsection{ABSTRACT}

This research investigated the removal capacity and kinetics of NIP for the removal of lead, copper, zinc, and nickel from synthetic and natural waters. NIP were tested for their ability to remove a mixture of lead, copper, zinc and nickel from both river water and wastewater. They were able to remove $82 \pm 0.2 \%$ of the lead, $46 \pm 0.6 \%$ of the copper, $55 \pm 20 \%$ of the zinc, and $17 \pm 2 \%$ of the nickel from a river water sample spiked with $500 \mathrm{ppb}$ of each heavy metal. Similarly, in a wastewater sample, they were able to remove $86 \pm 0.1 \%$ of the lead, $38 \pm 0.8 \%$ of the copper, $28 \pm 1 \%$ of the zinc, and $11 \pm 1 \%$ of the nickel. Competition with humic acid was investigated using synthetic natural water spiked with humic acid, and adsorption was compared to powdered activated carbon under similar conditions. $20 \mathrm{mg} / \mathrm{L}$ of humic acid was shown to limit the removal of heavy metals by adsorption for both NIP and activated carbon. Isotherm tests, conducted at $\mathrm{pH} 4$ in water, displayed a linear relationship between the free-lead remaining in solution and the adsorbent-phase concentration at equilibrium, and kinetics tests performed with lead, demonstrated that equilibrium was reached in less than 2 hours. 


\subsection{INTRODUCTION}

Wastewater effluents are the largest source of heavy metals in rivers and lakes (Ryan et al., 1982), and, due to increasing water stress, re-use of wastewater for irrigation is a major contributor to heavy metals in the food supply (Mapanda et al., 2005; Wang et al., 2005; Toze, 2006; Arora et al., 2008; Singh et al., 2010). The Canadian Drinking Water Guidelines set a maximum acceptable concentration of lead of $10 \mu \mathrm{g} / \mathrm{L}$ in treated drinking water. This concentration is derived from the concentration causing chronic health effects and therefore applies to average concentrations consumed over long periods of time (Health Canada, 2014). Heavy metals are typically removed from wastewater with ionexchange, membrane filtration (Kurniawan et al., 2006), or activated carbon adsorption (Fu and Wang, 2011). However, membrane filtration and ion-exchange processes can both have high capital and operational costs (Kurniawan et al., 2006), and activated carbons typically require chemical treatments to enhance their adsorption of heavy metals (Babel and Kurniawan, 2003).

Exposure to heavy metals can cause a variety of health effects depending on the heavy metal in question (Jarup, 2003). Heavy metals can accumulate in and affect the function of the heart, brain, kidneys, bones, liver, and other vital organs or they can displace essential nutrients required by the body (Singh et al., 2011). Cadmium can cause kidney damage, bone and skeletal damage, or renal failure, and is a possible human carcinogen (Jarup, 2003). Mercury can cause lung or kidney damage, or neurological and psychological symptoms (Jarup, 2003). Lead can cause acute symptoms such as headaches or irritability, behavioural disturbances in children, or neurological symptoms such as decreased memory 
(Jarup, 2003). Arsenic can be acutely fatal in large doses, and prolonged exposure through drinking water can lead to lung, bladder, and kidney cancer (Jarup, 2003).

Molecularly imprinted polymers (used for emerging contaminants), or ion-imprinted polymers (used for heavy metals) are designed to specifically target micropollutants. Molecularly imprinted polymers (MIP) are specific adsorbents for which polymerization is completed in the presence of a template. Following polymerization, the template is removed, and the resulting polymer particle possesses an affinity for the template molecule or molecules with similar structures (Masqué, 2001; Alexander, 2006). Ion imprinting is a similar process used to make polymers with an affinity for heavy metal ions, but typically require the addition of chelating agents. This is typically achieved either by incorporating chelating functional groups into the monomer, or by trapping chelating agents inside the polymer cavity during polymerization (Branger et al., 2013).

Non-imprinted polymers (NIP) are typically used as a reference material, against which MIP are tested, to investigate how specific the MIP are. However, the NIP themselves can also be used as non-specific adsorbents. In fact, for wastewater treatment purposes, specificity may not be the most important characteristic. An ideal treatment would target a variety of contaminants, but with limited interference from other wastewater constituents. This research investigated the use of NIP for removal of heavy metals from natural waters.

Methacrylic acid (MAA) was used as a monomer to create the NIP. MAA can also be used as a monomer for IIP, but typically requires the addition of chelating agents. These 
chelating agents are trapped inside the polymer cavity and form several interacting sites with the heavy metal of interest (Branger et al., 2013). In contrast, the NIP used in this study had no incorporated chelating agents or groups. When polymerized, MAA contains a carboxylic acid functional group, which is expected to adsorb heavy metals through an ion-exchange mechanism, but each MAA molecule contributes only one functional group and contributes a charge of -1 , whereas heavy metals typically have higher positive charges.

The goal of this research was to investigate the removal capacity and kinetics of NIP for the removal of lead, copper, zinc and nickel. NIP were tested for their ability to remove a mixture of lead, copper, zinc, and nickel from both river water and wastewater. In addition, synthetic natural water was created using humic acid, and was used to compare the performance of NIP to activated carbon during both simultaneous loading and pre-loading with humic acid. The kinetics of adsorption were established for adsorption of lead onto NIP, and lead isotherms, as well as isotherms for a mixture of lead, copper, zinc, and nickel, were evaluated after 14 days. These experiments were conducted over 14 days in contrast to those in Chapter 5 that were conducted over 4 days to evaluate the equilibrium behaviour of the NIP. The objective of this study was to determine whether or not NIP could quickly and effectively remove heavy metals from surface water and wastewater, and to compare the performance of the NIP with that of activated carbon. 


\subsection{MATERIALS AND METHODS}

\subsubsection{NIP synthesis}

Functional monomer methacrylic acid (MAA) (Sigma-Aldrich; Oakville, Canada) and cross-linker ethylene glycol dimethacrylate (EGDMA) (Sigma-Aldrich; Oakville, Canada) were dissolved in a porogen with a molar ratio of $8 \mathrm{mmol}: 6.7 \mathrm{mmol}$ (Wei et al., 2006). The porogen was composed of $40 \mathrm{~mL}$ of 1:3 (v:v) acetone (Fisher Scientific; Ottawa, Canada), and acetonitrile (Fisher; Ottawa, Canada). 2\%(w:w) of 2- isobutryonitrile (AIBN) was added as the initiator (Sigma-Aldrich; Oakville, Canada). The mixture was mixed with a vortex mixer (Fisher Scientific Vortex Mixer, USA), deoxygenated with nitrogen for five minutes, and then placed in a $60^{\circ} \mathrm{C}$ hot water bath for $24 \mathrm{~h}$ (Isotemp 220 , Fisher, USA). The resulting polymer particles were dewatered using a centrifuge (Thermo Scientific Sorval Legend $\mathrm{RT}^{+}$, Fisher Scientific) at 10,000 RPM, air dried at room temperature, and ground manually.

\subsubsection{Analytical measurements}

Heavy metal analysis was carried out at the ICP-MS facility at the University of Ottawa. Quantitative analysis of heavy metals was conducted with an Agilent 8800 triple quadrapole inductively coupled plasma mass spectrometer at an RF (radio frequency) power of $1500 \mathrm{~kW}$, gas flow of $1.07 \mathrm{~L} /$ minute, and sample uptake rate of $0.2 \mathrm{~mL} / \mathrm{minute}$. A total of 8 lab blanks were run prior to sample analysis, and lab standards were analyzed between each 10 samples. The detection limit for all four heavy metals was $4 \mathrm{ppt}$. 


\subsubsection{Experiments with environmental samples}

NIP were tested for their ability to remove lead, copper, nickel, and zinc from river water and wastewater samples. River water samples were collected from the Rideau River near Carleton University in Ottawa, Ontario. Secondary wastewater effluent samples were collected from the Robert O. Rickard Environmental Centre in Ottawa, Ontario. Appendix B provides summaries of the water quality for both water sampling locations. Water samples were not filtered, but controls with no NIP particles were used to eliminate effects from adsorption to suspended solids. Heavy metal standard solutions at concentrations of $1000 \mathrm{mg} / \mathrm{L}$ in nitric acid were purchased from Sigma-Aldrich. Two dilutions, one with 250 ppm of each metal and one with $10 \mathrm{ppm}$ of each metal were prepared in deionized water and neutralized with sodium hydroxide and hydrochloric acid as needed. The $\mathrm{pH}$ was measured using a Thermo Scientific Orion 5 Star $\mathrm{pH}$ meter.

River water and wastewater samples spiked with mixtures of $100 \mathrm{ppb}, 500 \mathrm{ppb}$, and 10, $000 \mathrm{ppb}$ of each heavy metal were prepared in $15 \mathrm{~mL}$ plastic centrifuge tubes. A $1000 \mathrm{mg} / \mathrm{L}$ NIP solution was created and sonicated (Vibracell Sonics, Sonics and Materials Inc, Newtown Connecticut) for 15 minutes to disperse the particles. $0.75 \mathrm{~mL}$ of the concentrated NIP solution was added to each tube to provide a final NIP concentration of $50 \mathrm{mg} / \mathrm{L}$. The samples were mixed on a shaker table (New Brunswick Scientific Excella E1 Platform Shaker, New Brunswick Scientific Inc.) for two weeks. They were then centrifuged (Thermo Scientific Sorval Legend RT+, Fisher Scientific) for 1 hour at a speed of $10,000 \mathrm{rpm}(670,800 \mathrm{~g})$. Following centrifugation, the centrate was carefully poured into new $15 \mathrm{~mL}$ centrifuge vials and the initial vials (containing the NIP particles) were 
discarded. Samples were acidified with $0.15 \mathrm{~mL}$ nitric acid (68-70\%, Anachemica, Montreal, Quebec) per $15 \mathrm{~mL}$ sample and stored at room temperature prior to analysis. Two experimental replicates were collected for each sample. PH was not monitored after initial sample preparation. Control samples were also prepared with the sample water alone, and sample water with NIP particles added.

\subsubsection{Experiments with synthetic water}

NIP were also tested and compared with a powdered activated carbon (PAC $200 \mathrm{M}-2018$, Norit, Cabot Canada, Sarnia, Ontario) for removal of the heavy metal mixture in deionized water spiked with humic acid. Refer to Appendix $\mathrm{C}$ for information from the manufacturer about the activated carbon. Norit PAC 200 is a coal-based activated carbon which was chosen for this purpose to maintain consistency with the experiments conducted in Chapter 7 of this thesis. Powdered activated carbon was used instead of granular activated carbon, because it has smaller particle sizes, which, although they are much larger than NIP, are closer in size than those of granular activated carbon. Sample water was created with 20 mg/L of Suwannee River (II) humic acid obtained from the International Humic Substances Society. A stock solution of the humic acid was prepared from the powdered humic acid and diluted to the final concentration. A detailed analysis of the humic acid, provided by the International Humic Substances Society, is available in Appendix D. The sample water was neutralized with sodium hydroxide (18\%) and hydrochloric acid (36.5-38\%). PH was not monitored after initial sample preparation.

Two sets of experiments were conducted: 
(1) NIP particles and activated carbon were pre-loaded with humic acid before exposure to heavy metals. Pre-loading experiments were conducted to investigate the extent of pore-blocking.

(2) NIP particles and activated carbon were simultaneously mixed with both humic acid and heavy metals. Simultaneous loading experiments were conducted to investigate the relative extents of direct competition and pore blocking. A decrease in adsorption for simultaneous loading is indicative of competition, and a further decrease for pre-loading is indicative of pore-blocking (Pelekani and Snoeyink, 1999).

Control samples were also prepared with the sample water alone, sample water with activated carbon added, and sample water with NIP particles added.

\subsubsection{Pre-loading experiments}

$1000 \mathrm{mg} / \mathrm{L}$ solutions of both NIP and activated carbon were produced as described above in Section 6.3.3 for the river water and wastewater samples. The particles were mixed with $15 \mathrm{~mL}$ of the humic acid sample at final adsorbent concentrations of $50 \mathrm{mg} / \mathrm{L}$ for the NIP and $10 \mathrm{mg} / \mathrm{L}$ for the activated carbon, for 24 hours on a shaker table. A 24 hour pre-loading period was chosen because it was not necessary for equilibrium adsorption of NOM to be achieved. Following the mixing period, the samples were centrifuged for 1 hour at 10,000 rpm. The centrate was discarded, and $100 \mathrm{ppb}, 500 \mathrm{ppb}$, and 10,000 ppb mixtures of the four heavy metals (lead, copper, zinc, and nickel) at $\mathrm{pH} 7$ were added. The samples were mixed with a vortex mixer (Fisher Scientific Vortex Mixer, USA) to distribute the particles, 
followed by a 2 week mixing period on a shaker table. At the end of the two weeks, samples were centrifuged and treated as outlined above in Section 6.3.3.

\subsubsection{Simultaneous loading experiments}

For the simultaneous loading experiments, the $20 \mathrm{mg} / \mathrm{L}$ of humic acid, $50 \mathrm{mg} / \mathrm{L}$ NIP or 10 $\mathrm{mg} / \mathrm{L}$ activated carbon, and $100 \mathrm{ppb}, 500 \mathrm{ppb}$, or 10,000 ppb heavy metal mixtures were combined simultaneously and treated as outlined above in Section 6.3.3.

\subsubsection{Adsorption isotherms and kinetics}

\subsubsection{Adsorption calculations and modelling}

\subsection{Percent removal by adsorption}

Percent removal was calculated based on the difference in concentrations between the control (containing no adsorbent) and the sample. The percent removal was calculated as shown in Equation 1, below.

$P_{A}(\%)=\frac{C_{S}-C_{C}}{C_{C}} \times 100,($ Equation 1)

Where:

$\mathrm{P}_{\mathrm{A}}=$ Percent removal by adsorption (\%)

$\mathrm{C}_{\mathrm{S}}=$ Liquid-phase sample concentration $(\mathrm{ppb})$ 
$\mathrm{C}_{\mathrm{C}}=$ Liquid-phase control concentration $(\mathrm{ppb})$

\subsection{Percent removal by other mechanisms}

In addition to removal by adsorption, some removal of heavy metals also occurred by other mechanisms such as NOM and heavy metal complexation, precipitation, or adsorption to the bottle. The percent difference between the concentration added and the measured concentration of the control sample was used to determine the percent removal, as shown in Equation 2, below.

$P_{O}(\%)=\frac{C_{C}-C_{i}}{C_{i}} \times 100,($ Equation 2$)$

Where:

$\mathrm{P}_{\mathrm{O}}=$ Percent removal by other mechanisms $(\%)$

$\mathrm{C}_{\mathrm{C}}=$ Liquid-phase control concentration $(\mathrm{ppb})$

$\mathrm{C}_{\mathrm{i}}=$ Initial liquid-phase concentration added $(\mathrm{ppb})$

\subsection{Adsorbent-phase concentration}

The amount adsorbed per mass of adsorbent, or the adsorbent-phase concentration, is an important parameter for evaluating adsorbent efficiency. The amount adsorbed, or percent 
removal, when not normalized by the mass of adsorbent, cannot be used to evaluate the efficiency of an adsorbent, because the amount adsorbed is dependent on the amount of adsorbent added. The adsorbent-phase concentration was calculated as shown in Equation 3 , below.

$q_{e}=\frac{C_{c}-C_{S}}{A}($ Equation 3)

Where:

$\mathrm{q}_{\mathrm{e}}=$ amount adsorbed $(\mu \mathrm{g} / \mathrm{mg}$ or $\mathrm{mg} / \mathrm{g})$

$\mathrm{A}=$ Adsorbent concentration (mg/L)

$\mathrm{C}_{\mathrm{s}}=$ Liquid-phase sample concentration $(\mu \mathrm{g} / \mathrm{L})$

$\mathrm{C}_{\mathrm{C}}=$ Liquid-phase control concentration $(\mu \mathrm{g} / \mathrm{L})$

\subsection{Langmuir adsorption isotherm}

The Langmuir adsorption isotherm is based on an assumption of a reversible chemical equilibrium between adsorbed adsorbate and free adsorbate. The Langmuir model is appropriate for adsorbents with homogeneous adsorbent surfaces each with equal energies of adsorption, with fixed adsorption sites, each able to adsorb one molecule of adsorbate. The Langmuir adsorption model can be written as: 
$q_{e}=\frac{Q_{M} b C_{s}}{1+b C_{s}}$, (Equation 4)

Where:

$\mathrm{q}_{\mathrm{e}}=$ amount adsorbed $(\mathrm{mg} / \mathrm{g})$

$\mathrm{Q}_{\mathrm{M}}=$ maximum adsorbent-phase concentration $(\mathrm{mg} / \mathrm{g})$

$\mathrm{b}=$ Langmuir adsorption constant $(\mathrm{L} / \mu \mathrm{g})$

$\mathrm{C}_{\mathrm{s}}=$ Liquid-phase sample concentration (ppb)

The Langmuir adsorption isotherm can be linearized as shown in Equation 5, below.

$\frac{C_{S}}{q_{e}}=\frac{1}{b Q_{M}}+\frac{C_{S}}{Q_{M}}$, (Equation 5)

\subsection{Freundlich adsorption isotherm}

The Freundlich adsorption isotherm model can be used to describe adsorption for heterogeneous adsorbents, where adsorption sites are not energetically equal. The Freundlich adsorption model is given by:

$q_{e}=K C_{s}^{1 / n},($ Equation 6)

Where: 
$\mathrm{q}_{\mathrm{e}}=$ amount adsorbed $(\mathrm{mg} / \mathrm{g})$

$\mathrm{K}=$ Freundlich adsorption capacity parameter $(\mathrm{mg} / \mathrm{g})(\mathrm{L} / \mu \mathrm{g})^{1 / \mathrm{n}}$

$1 / \mathrm{n}=$ Freundlich adsorption intensity parameter (unitless)

$\mathrm{C}_{\mathrm{s}}=$ Liquid-phase sample concentration $(\mathrm{ppb})$

The Freundlich isotherm can be linearized as shown in Equation 7, below.

$\log \left(q_{e}\right)=\log (K)+\left(\frac{1}{n}\right) \log \left(C_{s}\right),($ Equation 7)

\subsubsection{Adsorption kinetics for adsorption of lead onto NIP}

Experiments to establish the kinetics of adsorption were conducted in deionized water. The lead standard solution with $1000 \mathrm{mg} / \mathrm{L}$ of lead in nitric acid was diluted to $10,000 \mathrm{ppb}$ and adjusted to $\mathrm{pH} 4$ prior to addition to the sample tubes. Dilution water was created by acidifying deionized water to $\mathrm{pH}$ 4. Samples were prepared by filling $15 \mathrm{~mL}$ centrifuge tubes with $500 \mathrm{ppb}$ of lead and $50 \mathrm{mg} / \mathrm{L}$ NIP, using the acidified deionized water for dilution. The samples were placed on the shaker table and mixed. Samples were taken in duplicate following $1 \mathrm{~h}, 2 \mathrm{~h}, 5 \mathrm{~h}, 24 \mathrm{~h}, 2$ day, 5 day, and 7 day mixing periods. They were centrifuged and treated as outlined above for river water and wastewater. 


\subsubsection{Isotherm experiments for adsorption of lead onto NIP}

Lead isotherms were created following the procedure outlined above for kinetic experiments except that the NIP concentration was varied $(10 \mathrm{mg} / \mathrm{L}, 20 \mathrm{mg} / \mathrm{L}, 30 \mathrm{mg} / \mathrm{L}, 40$

$\mathrm{mg} / \mathrm{L}, 50 \mathrm{mg} / \mathrm{L}, 100 \mathrm{mg} / \mathrm{L}, 200 \mathrm{mg} / \mathrm{L}$, and $500 \mathrm{mg} / \mathrm{L}$ ) and the mixing time was kept constant at 14 days. A 14 day equilibration time was chosen because the kinetic and isotherm experiments were conducted at the same time, so the time to reach equilibrium was unknown, and excess time was provided to ensure that the samples would be at equilibrium.

\subsubsection{Isotherm experiments for adsorption of a mixture of heavy metals onto NIP}

Isotherms were also created for a mixture of lead, copper, zinc, and nickel. The procedure outlined above for lead isotherms was followed except that $500 \mathrm{ppb}$ each of lead, copper, zinc, and nickel were added.

\subsection{RESULTS AND DISCUSSION}

\subsubsection{Experiments with environmental samples}

\subsubsection{River water}

NIP were first tested for their ability to remove a mixture of four heavy metals (lead, copper, zinc, and nickel) from river water from the Rideau River in Ottawa, Ontario. The Rideau River comes from a watershed with an area of $3.935 \mathrm{~km}^{2}$ that is predominately forested (44\%), and agricultural (38\%), and was chosen because it contains relatively few wastewater inputs (City of Ottawa, 2006). The $\mathrm{pH}$ of the river water was 7.56. Three different initial heavy metal concentrations (100 ppb, $500 \mathrm{ppb}$, and 10,000 ppb) were spiked into the river water along with $50 \mathrm{mg} / \mathrm{L}$ of NIP, and allowed to equilibrate for 2 
weeks. The maximum acceptable concentration for lead in drinking water established by the Canadian Drinking Water Guidelines is $10 \mathrm{ppb}$, and $100 \mathrm{ppb}$ represents a high concentration of lead for surface waters, but could represent a highly polluted surface water (Health Canada, 2014). The $500 \mathrm{ppb}$ concentration was chosen to represent a wastewater effluent concentration, and the $10,000 \mathrm{ppb}$ concentration was chosen to represent industrial wastewater or highly polluted waters to evaluate the limits of treatment. Figure 6-1 (a) presents the removal by mechanisms other than removal on NIP particles, and was calculated based on the difference between the concentrations added, and the concentrations of the control samples containing no NIP. Figures 6-1 (b) and (c) represent the percent removal and equilibrium adsorbent phase concentration (y-axis) versus the initial heavy metal concentration (x-axis), respectively. Percent removal was plotted in addition to adsorbent loading to clearly show the efficiency of the particles over a wide range of initial concentrations. Both the percent removal and amount adsorbed were calculated by comparing the equilibrium aqueous phase concentrations to blanks with no NIP particles. This was done to exclude the removal by other mechanisms, shown in Figure 6-1 (a), such as complexation by filterable organic materials, precipitation, or adsorption to the sample tubes from the calculation of the amount adsorbed. 


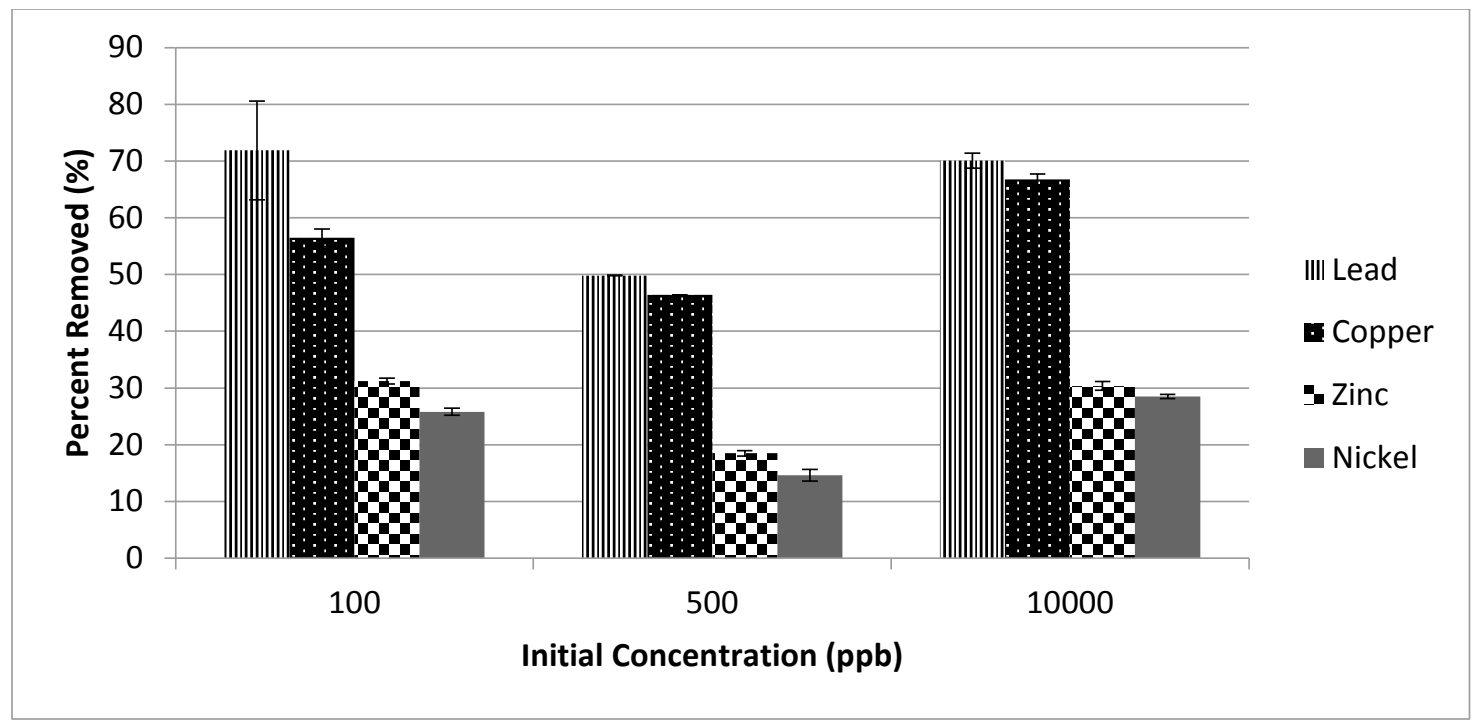

Figure 6-1 (a): Percent removed via mechanisms other than adsorption by NIP versus initial heavy metal concentration for lead, copper, zinc, and nickel in river water

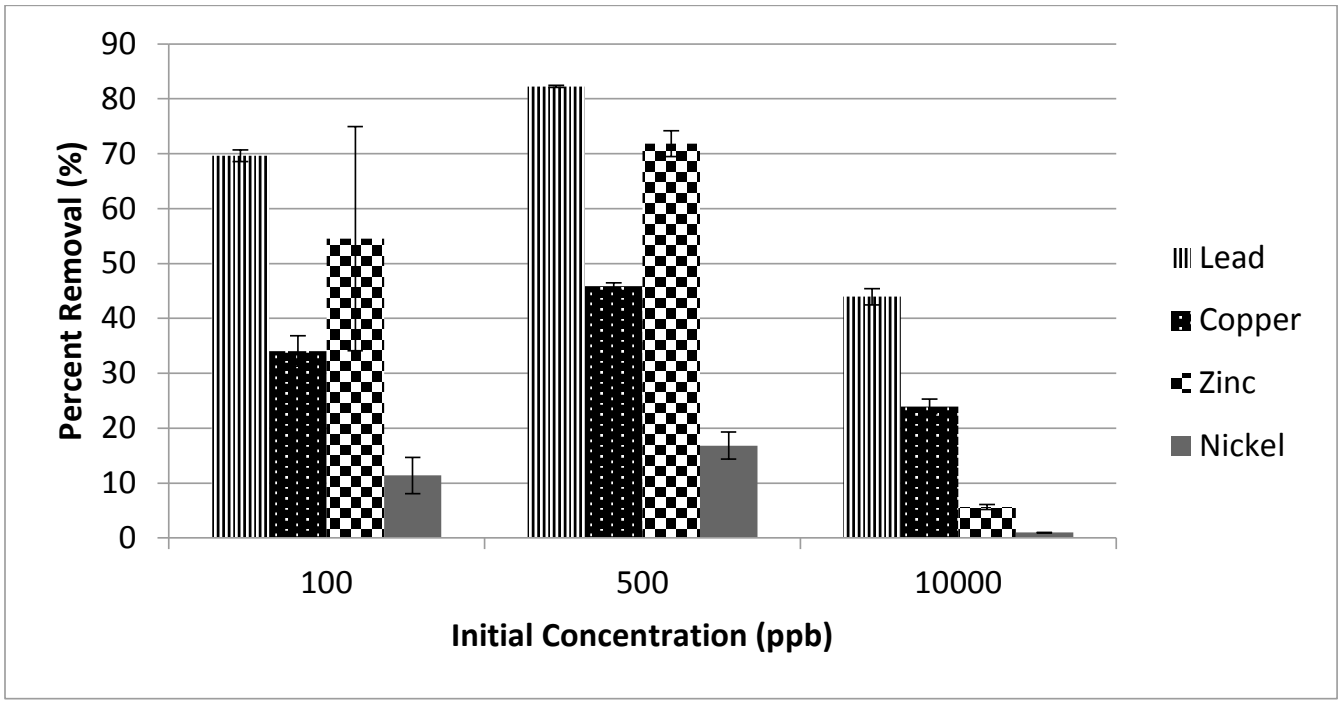

Figure 6-1 (b): Percent removal by NIP versus initial heavy metal concentration for lead, copper, zinc, and nickel in river water 


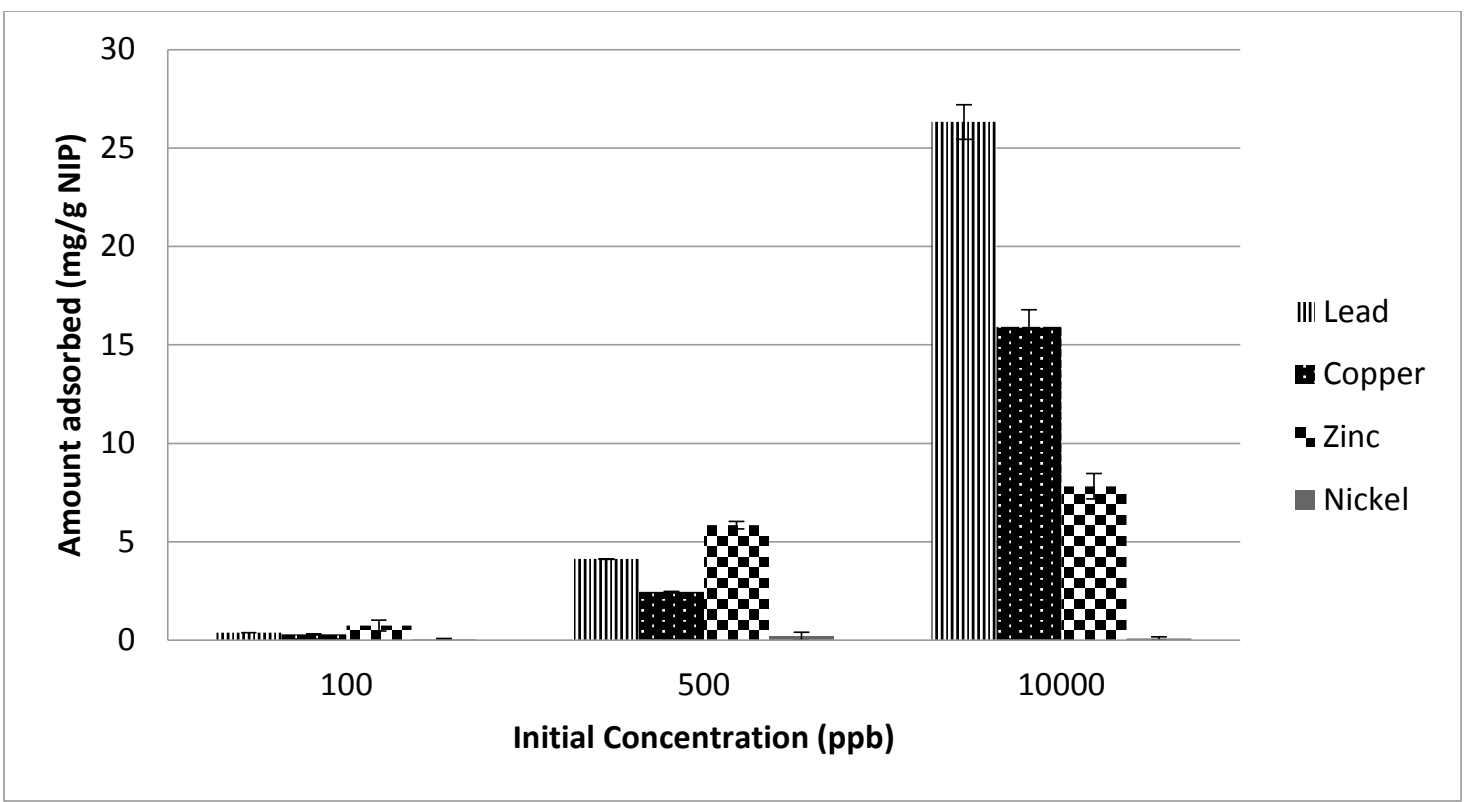

Figure 6-1 (c): Amount adsorbed by NIP versus initial heavy metal concentration for lead, copper, zinc, and nickel in river water

Figure 6-1 (b) indicates some removal of all four heavy metals. The percent removal for lead increased from $70 \pm 1 \%$ to $82 \pm 0.2 \%$ as the initial concentration of heavy metals increased from $100 \mathrm{ppb}$ to $500 \mathrm{ppb}$, before decreasing to $44 \pm 1 \%$ for $10,000 \mathrm{ppb}$. Figure 6-1 (c) shows the corresponding adsorbent-phase concentrations, which increased from $0.39 \pm 0.006 \mathrm{mg} / \mathrm{g}$ for $100 \mathrm{ppb}$ to $4.13 \pm 0.008 \mathrm{mg} / \mathrm{g}$ for $500 \mathrm{ppb}$ and $26 \pm 0.9 \mathrm{mg} / \mathrm{g}$ for $10,000 \mathrm{ppb}$. This indicates that the particles had not reached adsorbent-phase saturation at $500 \mathrm{ppb}$. An additional experimental concentration is required to determine if the particles had reached saturation at 10,000 ppb because a constant adsorbent-phase concentration with increasing initial concentration is required to confirm saturation.

Likewise, for copper, the percent removal increased from $34 \pm 3 \%$ for 100 ppb to $46 \pm 0.6$ $\%$ for $500 \mathrm{ppb}$, before decreasing to $24 \pm 1 \%$ for $10,000 \mathrm{ppb}$. Like lead, the adsorbent- 
phase concentration continued to increase as the concentration of heavy metals increased. The adsorbent-phase concentration increased from $0.3 \pm 0.002 \mathrm{mg} / \mathrm{g}$ for $100 \mathrm{ppb}$ to $2.46 \pm$ $0.03 \mathrm{mg} / \mathrm{g}$ for $500 \mathrm{ppb}$ and $16 \pm 0.9 \mathrm{mg} / \mathrm{g}$ for $10,000 \mathrm{ppb}$. Again, it is not possible to conclusively state whether the particles reached saturation.

Zinc also exhibited an increasing percent removal between 100 and $500 \mathrm{ppb}$, from $55 \pm$ $20 \%$ to $72 \pm 2 \%$. There was a large difference between sample replicates for the $100 \mathrm{ppb}$ initial concentration, which makes it difficult to discern whether or not there was a real difference, but the amount adsorbed, shown in Figure 6-1 (c), also increased. The percent removal then decreased to $6 \pm 0.5 \%$ for an initial concentration of $10,000 \mathrm{ppb}$. The adsorbent-phase concentration followed a similar trend to that already discussed for lead and copper. The adsorbent-phase concentration increased from $0.8 \pm 0.3 \mathrm{mg} / \mathrm{g}$ for $100 \mathrm{ppb}$ to $5.9 \pm 0.2 \mathrm{mg} / \mathrm{g}$ for $500 \mathrm{ppb}$ and $7.8 \pm 0.6 \mathrm{mg} / \mathrm{g}$ for $10,000 \mathrm{ppb}$. Again, it is not possible to conclude whether or not the particles were fully saturated for initial concentrations greater than $500 \mathrm{ppb}$.

Nickel followed a similar trend to lead, copper, and zinc for percent removal shown in Figure 6-1 (b), but a somewhat different trend for the adsorbent-phase concentration shown in Figure 6-1 (c). Figure 6-1 (b) indicates an increase in percent removal from $11 \pm 3 \%$ for $100 \mathrm{ppb}$ to $17 \pm 2 \%$ for $500 \mathrm{ppb}$ and a decrease to $1 \pm 0.06 \%$ for $10,000 \mathrm{ppb}$. The adsorbentphase concentration increased from $0.17 \pm 0.05 \mathrm{mg} / \mathrm{g}$ for $100 \mathrm{ppb}$ to $1.4 \pm 0.2 \mathrm{mg} / \mathrm{g}$ for 500 $\mathrm{ppb}$ and remained constant at $1.4 \pm 0.09 \mathrm{mg} / \mathrm{g}$ for $10,000 \mathrm{ppb}$. The constant value of the adsorbent-phase concentration indicates that the particles reached saturation for an initial 
liquid phase concentration between 100 and $500 \mathrm{ppb}$. This is interesting, because nickel was tested as part of a mixture of other heavy metals, which did not reach saturation for $500 \mathrm{ppb}$. This indicates that the other metals may have out-competed nickel at higher concentrations or nickel may have had access to fewer adsorption sites.

\subsection{Water chemistry and adsorption of heavy metal ions}

From Figures 6-1 (b) and (c), it is clear that the NIP showed a greater affinity for some heavy metals over others, as indicated by a higher level of adsorption for those metals. The system, which included the NIP and heavy metal ions, but also the river water with dissolved and suspended organic and inorganic matter, and the sample tubes, was complex. Heavy metal ions may have formed complexes with natural organic matter (NOM) present in the river water, or reacted with alkalinity and precipitated out of solution, as well as adsorbing to the NIP particles.

The NIP prepared for this study contained carboxylic acid functional groups, and possessed a negative surface charge under neutral conditions (Lai et al., 2010). Caroxylic acid functional groups on the NIP surface can act through ion-exchange, exchanging $\mathrm{H}^{+}$ions with heavy metal ions. Polymeric ion exchangers get their selectivity from the stereochemistry of functional groups on the polymer surface, which is fixed due to the rigid structure provided by the cross-linker. The heavy metal ion can bind with at most two oxygen atoms from neighbouring carboxylic acid groups, and the preferred spatial orientation of the metal-functional group complex depends on the metal ions coordination number. The selectivity series, or order of preference for heavy metal ions is generally the 
same for ion-exchangers with the same functional groups, and for carboxylic acid functional groups is: $\mathrm{Pb}>\mathrm{Cu}>\mathrm{Zn}>\mathrm{Ni}$ (SenGupta, 2002). From Figure 6-1 (c), the selectivity sequence was $\mathrm{Zn}>\mathrm{Pb}>\mathrm{Cu}>\mathrm{Ni}$ for initial concentrations of $100 \mathrm{ppb}$ and $300 \mathrm{ppb}$, and $\mathrm{Pb}>\mathrm{Cu}>\mathrm{Zn}>\mathrm{Ni}$ for an initial concentration of $10,000 \mathrm{ppb}$. Therefore the selectivity series for $10,000 \mathrm{ppb}$ followed that expected for carboxylic acid ion exchangers, but those for $100 \mathrm{ppb}$ and $500 \mathrm{ppb}$ did not. These differences were likely due to competitive effects of heavy metal-NOM complexes and precipitation.

NOM present in natural waters contains carboxyl, phenol, sulfhydryl, and amine functional groups which will compete with the carboxyl functional groups on the NIP for heavy metals. Generally, heavy metals form stable complexes with NOM, which increases the solubility of the heavy metals by reducing the metal reactivity. Complexed heavy metals are generally less reactive than free heavy metal ions present at the same concentration, and are less likely to react with carbonates in solution and precipitate out of solution (Benjamin, 2002). Since ion-exchange reactions with NOM were competing directly with the ion-exchange reaction on the NIP, it is possible that NOM-lead complexes, formed before the addition of the NIP, out-competed NIP for complexation with lead. This may have led to NIP adsorbing more zinc than lead for initial concentrations of $100 \mathrm{ppb}$ and $500 \mathrm{ppb}$. For $10,000 \mathrm{ppb}$, available ion-exchange sites on the NOM may have been filled, leaving more lead available for adsorption to the NIP.

Natural waters contain carbonate species due to reactions with air. In natural waters under neutral conditions, the probable main species of the heavy metals studied are: $\mathrm{PbCO}_{3}$ and 
$\mathrm{Pb}\left(\mathrm{CO}_{3}\right)_{2}{ }^{-2}$ for lead, $\mathrm{CuCO}_{3}$ and $\mathrm{CuOH}^{+}$for copper, $\mathrm{ZnCO}_{3}$ and $\mathrm{ZnOH}^{+}$for zinc, and $\mathrm{Ni}^{2+}$ and $\mathrm{NiCO}_{3}$ for nickel. Metal carbonates are typically insoluble and precipitate from solution (Stumm and Morgan, 1981). For these experiments, precipitation was accounted for with the control samples, which contained river water spiked with heavy metals, and which were prepared following the same procedure as the samples containing the NIP. The percent removals and amounts adsorbed shown in Figure 6-1 (b) and (c) were determined by calculating the amount removed by the NIP based on the difference between the samples containing NIP and the controls. Figure 6-1 (a) shows the amount removed by other mechanisms, which was high, especially for lead and copper. It is likely that most of the removal by other mechanisms was due to reactions with alkalinity and subsequent precipitation. It is also possible for the addition of the NIP to enhance precipitation by providing nucleation sites for precipitation to occur. It is not possible to account for this based on the experimental design, and, removal by the NIP may have included both removal through ion exchange and through precipitation onto the surface of the NIP.

\subsubsection{Wastewater}

NIP were also tested for their capacity to remove a mixture of heavy metals from wastewater. A secondary effluent sample was taken prior to disinfection from the Robert O. Pickard Environmental Centre in Ottawa, Ontario. The $\mathrm{pH}$ of the wastewater sample was 8.01 . Figure 6-2 (a) was calculated based on the difference between the concentrations of metals added, and the concentrations of the controls, and provides percent removal values for removal via other mechanisms in absence of NIP. Figures 6-2 (b) and (c) represent the percent removal and equilibrium adsorbent phase concentrations (y-axis) 
versus the initial concentration ( $\mathrm{x}$-axis) for samples containing NIP, respectively. The results shown represent the averages of two replicates, and the error bars represent the maximum and minimum values.

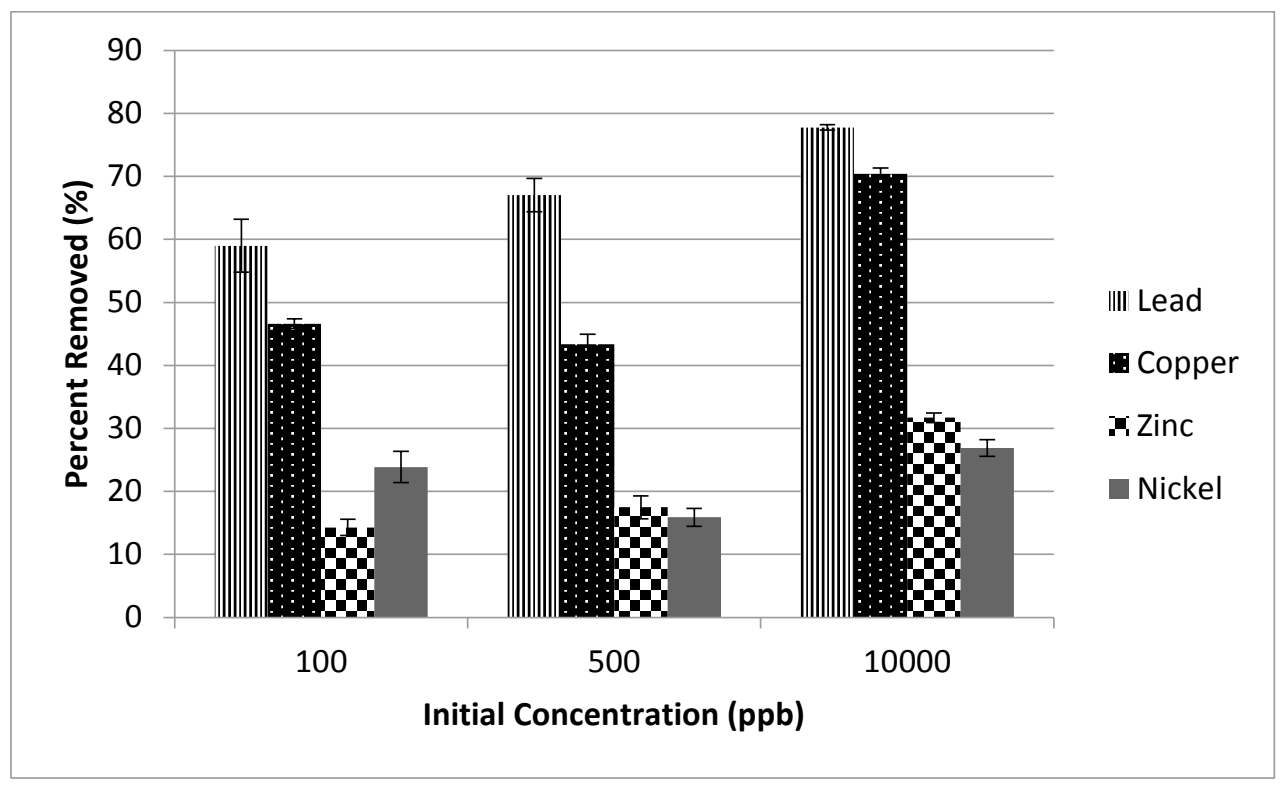

Figure 6-2 (a): Percent removal via mechanisms other than adsorption by NIP versus initial heavy metal concentration for lead, copper, zinc and nickel in wastewater 


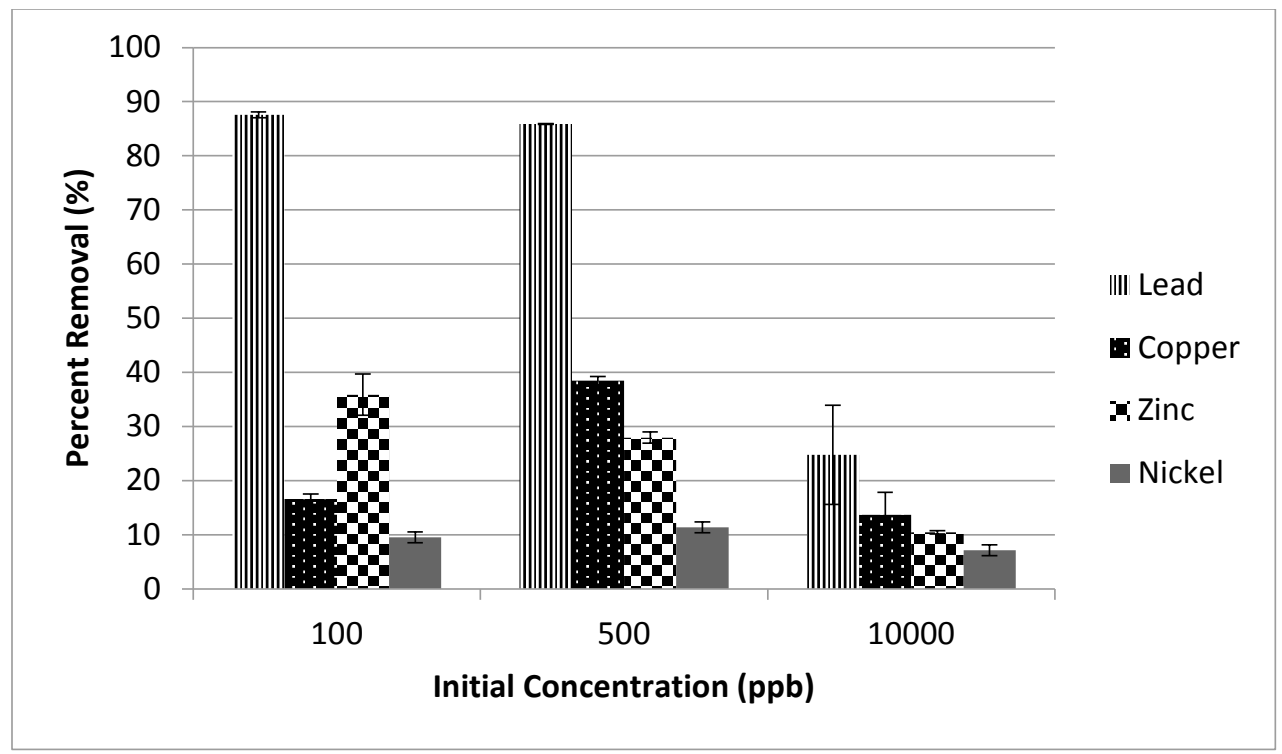

Figure 6-2 (b) Percent removal by NIP versus initial heavy metal concentration for lead, copper, zinc, and nickel in wastewater

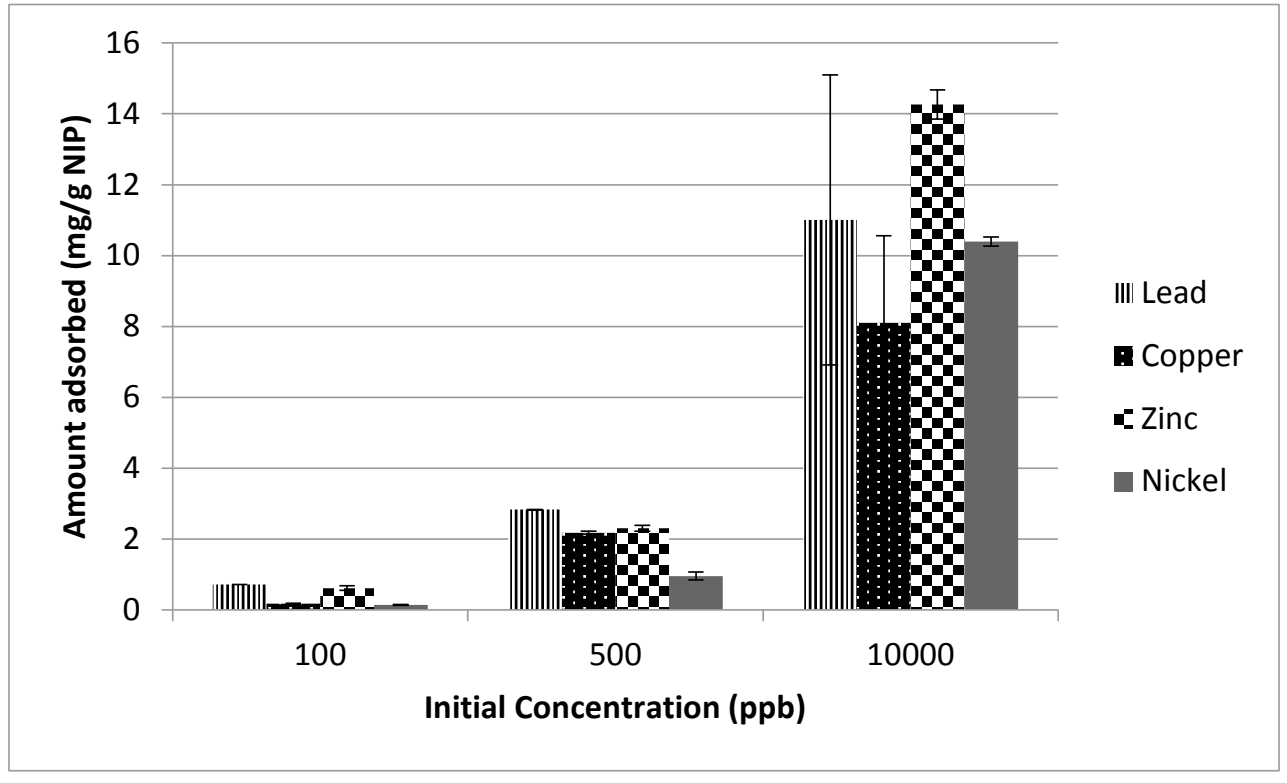

Figure 6-2 (c): Amount adsorbed by NIP versus initial heavy metal concentration for lead, copper, zinc and nickel in wastewater 
Figure 6-2 (b) provides percent removal values for removal of lead, copper, zinc, and nickel from wastewater. In general, the removal of each heavy metal was less than that shown in Figure 6-1 (b) for river water, with a few notable exceptions. Lead exhibited a higher percent removal in wastewater for concentrations of 100 and $500 \mathrm{ppb}$, and zinc and nickel were both removed to a greater extent in wastewater for an initial concentration of 10,000 ppb. It can be assumed that the wastewater contained greater concentrations of NOM, generally lowering adsorption of heavy metals onto the NIP particles.

Figure 6-2 (b) shows that the percent removal for lead decreased from $87.5 \pm 0.5 \%$ for 100 ppb to $85.9 \pm 0.1 \%$ for $500 \mathrm{ppb}$, and $24.8 \pm 0.5 \%$ for $10,000 \mathrm{ppb}$. Figure $6-2$ (c) provides the corresponding adsorbent-phase concentrations, which increased from $0.72 \pm 0.005$ $\mathrm{mg} / \mathrm{g}$ for $100 \mathrm{ppb}$ to $2.83 \pm 0.002 \mathrm{mg} / \mathrm{g}$ for $500 \mathrm{ppb}$ and $11 \pm 4.1 \mathrm{mg} / \mathrm{g}$ for $10,000 \mathrm{ppb}$. Interestingly, the amount of lead adsorbed from the wastewater was higher than for river water for the $100 \mathrm{ppb}$ sample, but lower for $500 \mathrm{ppb}$ and $10,000 \mathrm{ppb}$, but the percent removal was greater for both $100 \mathrm{ppb}$ and $500 \mathrm{ppb}$. This difference can be explained by looking at the percent removal via other mechanisms for wastewater, shown in Figure 6-2 (c), in comparison to that for river water in Figure 6-1 (a). Competitive removal was higher for river water for $100 \mathrm{ppb}$, but higher for wastewater for $100 \mathrm{ppb}$ and 10,000 ppb, making comparisons with percent removal difficult.

The percent removal for copper, shown in Figure 6-2 (b), was lower than that in the river water (Figure 6-1 (b)), for all three concentrations. The percent removal for copper increased from $17 \pm 1 \%$ for $100 \mathrm{ppb}$ to $38 \pm 0.8 \%$ for $500 \mathrm{ppb}$ prior to decreasing to $14 \pm$ 
$4 \%$ for 10,000 ppb. Adsorbent-phase concentrations, shown in Figure 6-2 (c), increased throughout this range, starting at $0.18 \pm 0.01 \mathrm{mg} / \mathrm{g}$ for $100 \mathrm{ppb}$, and increasing to $2.18 \pm$ $0.04 \mathrm{mg} / \mathrm{g}$ for $500 \mathrm{ppb}$ and $8.10 \pm 2.46 \mathrm{mg} / \mathrm{g}$ for $10,000 \mathrm{ppb}$. It is not possible to conclude whether the adsorption sites on the NIP became saturated for copper over this range. The adsorbent-phase concentrations for $100 \mathrm{ppb}$ and $500 \mathrm{ppb}$ were similar to those recorded for the river water sample (Figure 6-2 (c)), but that for the 10,000 ppb sample was approximately half the concentration observed for river water. This behaviour indicates that copper ions may have experienced competition for adsorption sites at high concentrations or have become saturated at a lower adsorbent-phase concentration in the wastewater.

The percent removal for zinc was higher for the river water sample (Figure 6-1 (b)) for 100 and $500 \mathrm{ppb}$, and higher for the wastewater sample for $10,000 \mathrm{ppb}$. Percent removal of zinc decreased from $36 \pm 4 \%$ for $100 \mathrm{ppb}$ to $28 \pm 1 \%$ for $500 \mathrm{ppb}$, and $10 \pm 0.3 \%$ for 10,000 ppb. The amount adsorbed over this range increased from $0.61 \pm 0.07 \mathrm{mg} / \mathrm{g}$ for $100 \mathrm{ppb}$ to $2.3 \pm 0.08 \mathrm{mg} / \mathrm{g}$ for $500 \mathrm{ppb}$ and $14 \pm 0.4 \mathrm{mg} / \mathrm{g}$ for $10,000 \mathrm{ppb}$. The amount adsorbed was less than that for river water for concentrations of 100 and $500 \mathrm{ppb}$, but nearly twice that observed for the river water sample for $10,000 \mathrm{ppb}$.

Similar to zinc, the percent removal for nickel in river water (shown in Figure 6-1 (b)) was higher than for river water (shown in Figure 6-2 (b)) for 100 and 500 ppb, but higher for the wastewater sample for an initial concentration of 10,000 ppb. This is likely because of competition from the NOM in wastewater. The percent removal for nickel from wastewater 
increased from $10 \pm 0.8 \%$ to $11 \pm 1 \%$ from 100 to $500 \mathrm{ppb}$, before decreasing to $7 \pm 0.1 \%$ for $10,000 \mathrm{ppb}$; a decrease which was less pronounced in the wastewater than the river water. The adsorbent-phase concentration increased in this range, and did not remain constant for the highest concentrations as it did for the river water. As previously noted, a constant adsorbent-phase concentration with increasing initial concentration, indicates that the adsorbent has no additional capacity for the adsorbate. For the river water sample, this occurred for initial concentrations greater than $500 \mathrm{ppb}$, but there was no evidence of saturation for the wastewater sample. In fact, the adsorbent-phase concentration for the wastewater for an initial concentration of $10,000 \mathrm{ppb}$ was more than 7 times that observed for the river water sample. The adsorbent-phase concentration increased from $0.14 \pm 0.01$ $\mathrm{mg} / \mathrm{g}$ for $10,000 \mathrm{ppb}$ to $0.96 \pm 0.1 \mathrm{mg} / \mathrm{g}$ for $500 \mathrm{ppb}$ and $10.4 \pm 0.1 \mathrm{mg} / \mathrm{g}$ at $10,000 \mathrm{ppb}$. Heavy metal-organics complexation on the surface of NIP in wastewater may have increased adsorption.

\subsubsection{Experiments with synthetic water}

As previously stated, both the river water and wastewater samples were complex systems containing unknown compositions of ions, organic materials, particles, and other dissolved and suspended constituents. They were real water samples containing the types of complexities that might be encountered while using NIP for water or wastewater treatment. However, to fully understand the mechanisms behind what was occurring, a simpler matrix was needed. Synthetic natural water samples were created for this purpose using deionized water spiked with $20 \mathrm{mg} / \mathrm{L}$ of humic acid. The humic acid used was Suwannee River Humic Acid Standard (II) purchased from the International Humic Substances Society. 
Information about the composition and properties of the humic acid is provided in Appendix D. For these studies, removal of heavy metals with NIP was compared to removal with a powdered activated carbon (PAC 200 from Norit). NIP and activated carbon were compared because activated carbon is currently the most commonly used adsorbent in water and wastewater treatment.

Two sets of experiments were completed. In the first, deionized water was spiked with the humic acid standard and neutralized before $50 \mathrm{mg} / \mathrm{L}$ of NIP particles or $10 \mathrm{mg} / \mathrm{L}$ of activated carbon were added. The adsorbents were allowed to contact the humic acid for 24 hours before being centrifuged. The centrate was discarded, and the particles, containing adsorbed humic acid, were saved. Deionized water spiked with varying heavy metal concentrations at a neutral $\mathrm{pH}$ was then added to the tubes with the particles, mixed well, and allowed to equilibrate for 2 weeks. For the second set of experiments, the humic acid, heavy metals, and adsorbents were added and mixed in one step. This was done to discern whether or not the humic acid blocked access to pores in the activated carbon or NIP, or competed directly for adsorption sites. If the pores were blocked, the pre-loaded samples would be expected to exhibit lower adsorption because heavy metal ions would have no exposure to pores prior to blocking by the humic acid. If humic acid and heavy metal ions competed directly for access to adsorption sites, pre-loading with humic acid would not be expected to have an additional effect. (Pelekani and Snoeyink, 1999)

Figures 6-3 (a) and (b) both show that there was negligible removal (less than $6 \pm 2 \%$ ) for all heavy metals at all concentrations. This makes it difficult to determine whether 
significant differences existed between the pre-loaded and simultaneously loaded samples. It also makes it difficult to determine a selectivity series for comparison with the river water and wastewater samples. Figure 6-3 (c), shows removal by other mechanisms, which may have included NOM and heavy metal complexation, precipitation, adsorption to the bottle, or adsorption and subsequent precipitation with humic acid. From Figure 6-3 (c), it is clear that while the NIP did not significantly remove any of the heavy metals during either simultaneous exposure with humic acid or pre-loading, the NIP were still removed by other adsorption mechanisms.

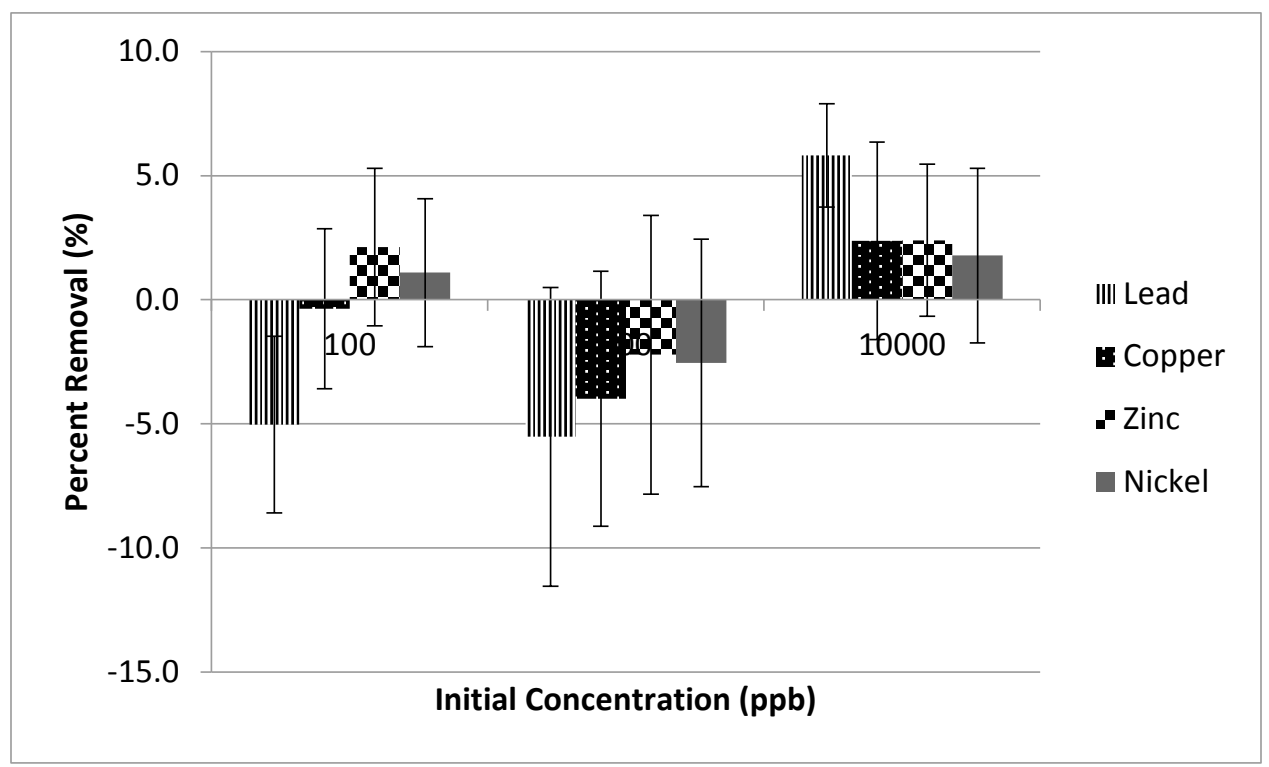

Figure 6-3 (a): Adsorption of lead, copper, zinc, and nickel on NIP pre-loaded with humic acid 


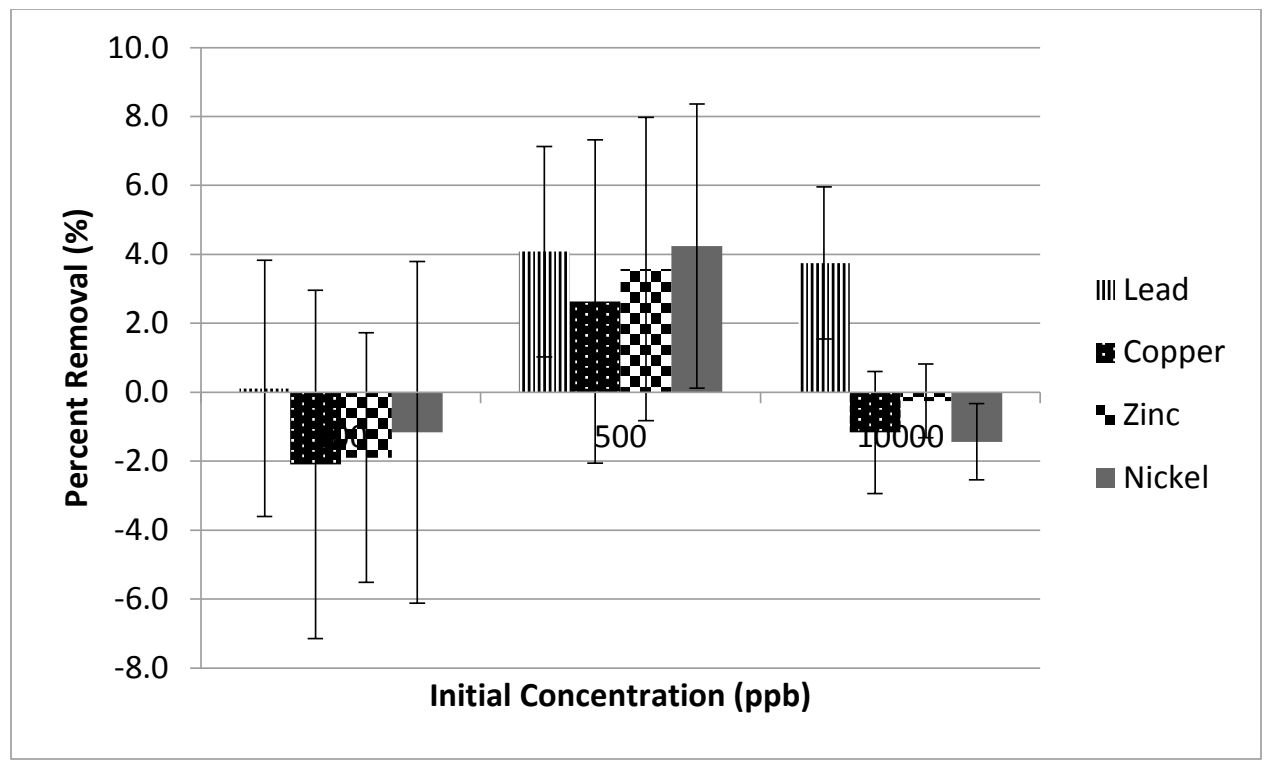

Figure 6-3 (b): Adsorption of lead, copper, zinc, and nickel on NIP simultaneously loaded with humic acid

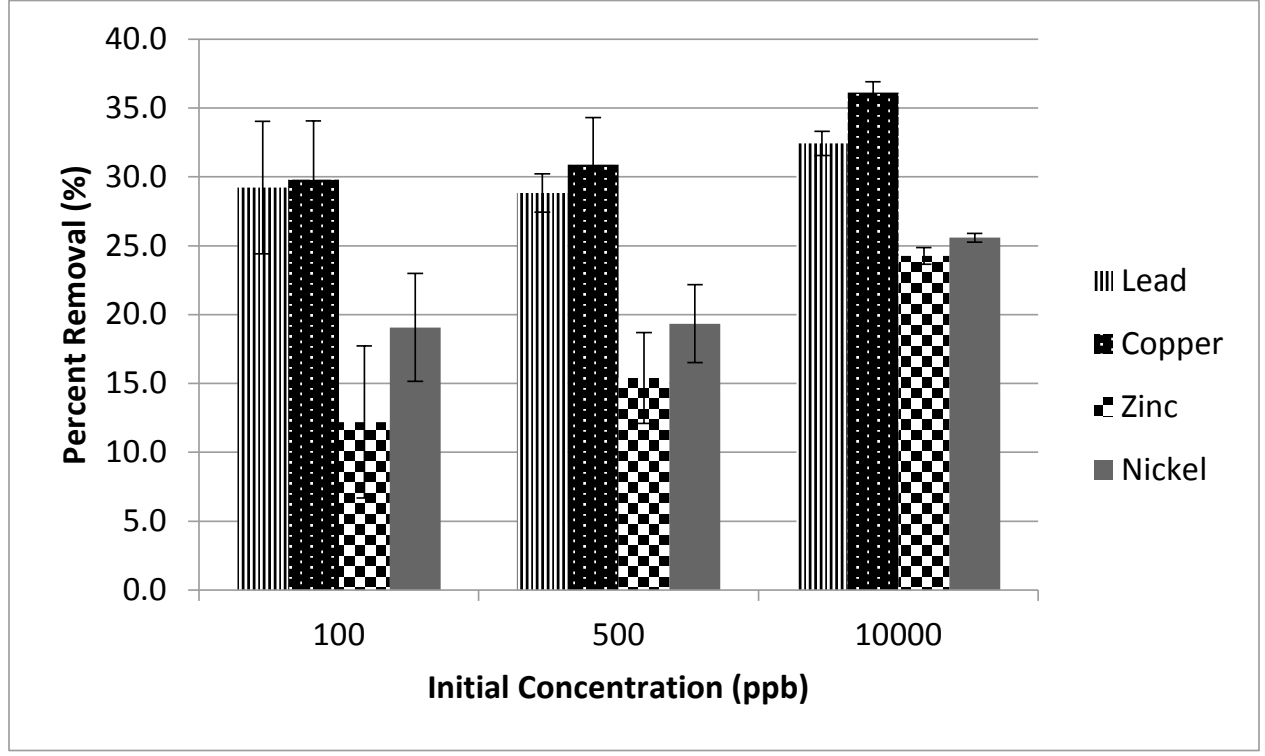

Figure 6-3 (c): Percent removed via mechanisms other than adsorption by NIP particles or activated carbon versus initial heavy metal concentration for lead, copper, zinc and nickel in wastewater 
The pre-loaded and simultaneously-loaded activated carbon results are provided in Figures 6-4 (a) and (b), respectively. Adsorption of heavy metals onto the activated carbon was not significant in the presence of humic acid and it was difficult to discern an effect from preloading due to large error bars. Low removal was likely due to the formation of humic acid - heavy metal complexes which were stable in solution. For lead and copper at initial concentrations of $100 \mathrm{ppb}$ each, the simultaneously loaded activated carbon exhibited slightly higher removal. For the pre-loaded sample, the percent removal was $-10 \pm 2 \%$ for lead and $-4.1 \pm 3.2 \%$ for copper at $100 \mathrm{ppb}$. Negative percent removal values can be attributed to experimental error, indicating that there was zero to negligible removal for the pre-loaded sample. A control sample was prepared with activated carbon in the sample water, but without spiked heavy metals, and indicated that there was no leaching of heavy metals from the activated carbon. The simultaneously loaded sample had corresponding percent removals of $6 \pm 12 \%$ for lead and $5.3 \pm 2.3 \%$ for copper at the same concentration. The percent removal values were too low and the variability was too high to state conclusively whether or not there was an effect from pore blocking. Figure 6-3 (c) contained no NIP or activated carbon, and was used as a control for both adsorbents. As shown in Figure 6-3 (c), there was significant removal of each of the heavy metals by other mechanisms, which may have included adsorption to settleable humic acid, adsorption to the sample tubes, or precipitation. The removal by other mechanisms shown in Figure 6-3 (c) was lower than that observed for river water and wastewater, shown in Figures 6-1(c) and 6-2 (c), respectively. This was probably due to a lower concentration of carbonate species in the deionized water versus the natural water samples which had more time to 
equilibrate with air, and reduced precipitation. Copper had the highest percent removal by other mechanisms followed by lead, nickel, and zinc for all three concentrations studied.

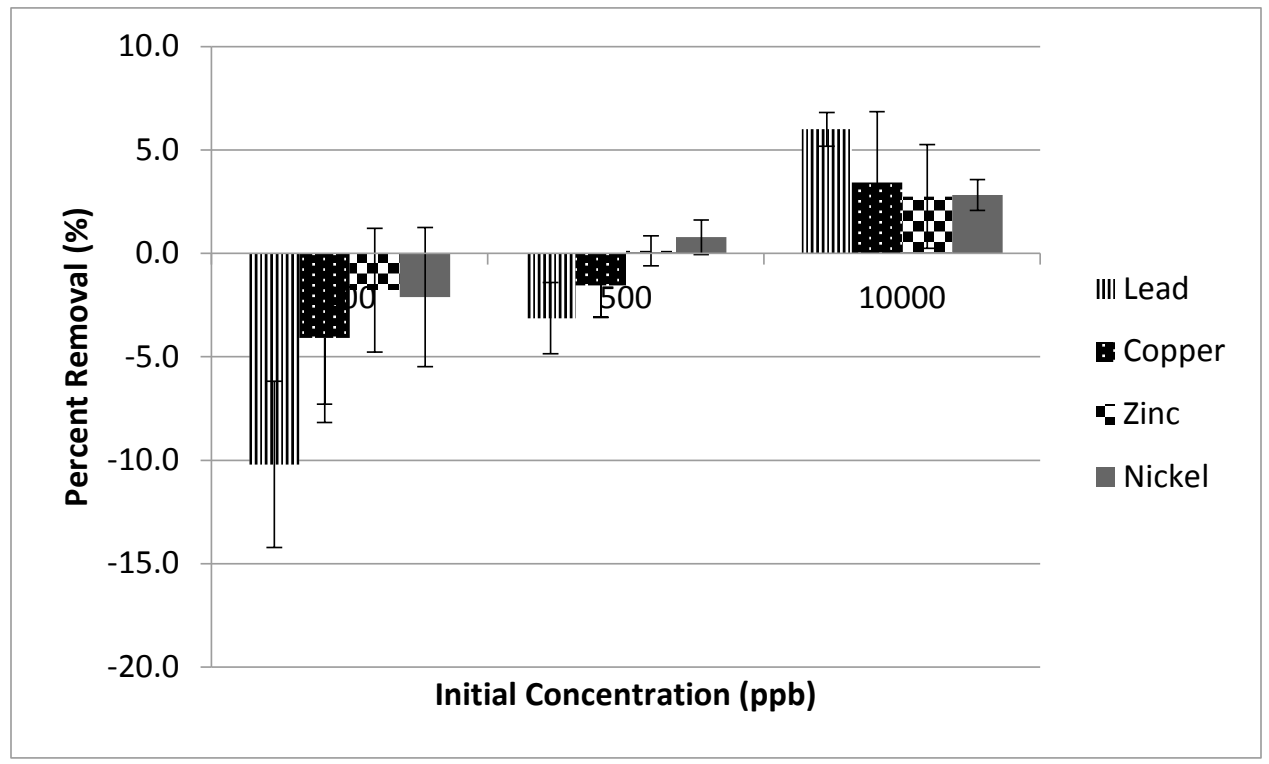

Figure 6-4 (a): Adsorption of lead, copper, zinc, and nickel on activated carbon preloaded with humic acid

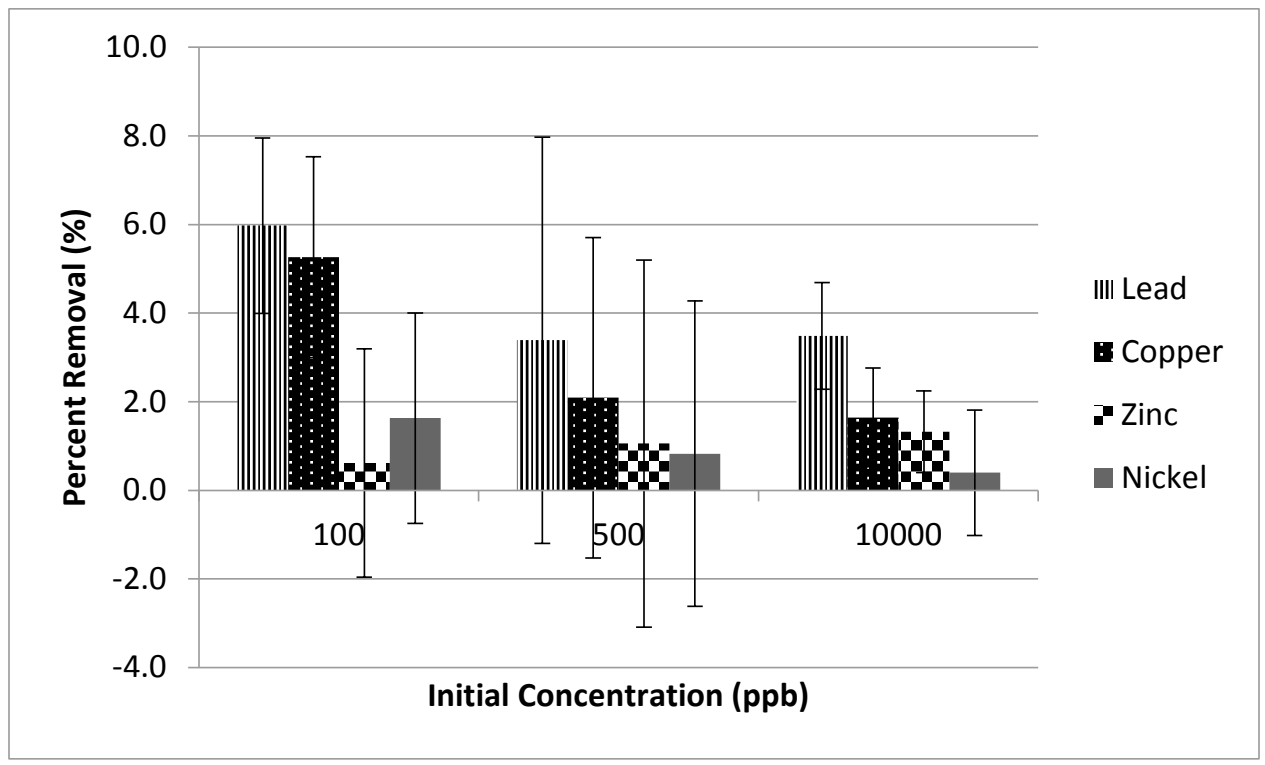


Figure 6-4 (b): Adsorption of lead, copper, zinc and nickel on activated carbon simultaneously loaded with humic acid

It is likely that for both the NIP and the activated carbon, the humic acid competed to such a degree that there was negligible adsorption of the heavy metals. It was hypothesized herein that pre-loaded samples would experience decreased adsorption due to both increased pore blocking and direct competition, whereas the simultaneously loaded samples would predominately experience direct competition with more limited pore blocking. Heavy metal ions are very small in size, and are not expected to experience direct competition from humic acid, which has a much larger molecular size (Ebie et al, 2001). However, both direct site competition and pore blocking can occur during simultaneous loading, and pore blocking of the very small pores favoured by heavy metal ions may have occurred to such an extent that there was limited adsorption of heavy metal ions for either sample, and it was not possible to observe any additional decrease caused by pore blocking during pre-loading. It is also possible that there was another mechanism reducing adsorption, such as the presence of heavy metal - humic acid complexes, which may have also affected the diffusion of heavy metals into pores. Competition with humic acid was not expected to have such an extensive effect on the NIP and activated carbon, and should be studied further to determine why competition with humic acid led to such large decreases in adsorption capacity. Al- Attas et al (2012) studied the effects of NOM-iron complexes on adsorption of iron with GAC, and found that the presence of NOM decreased adsorption by a factor of 7 in comparison to organic-free water, and found that the decrease was largely due to competition for adsorption sites and not NOM-iron complexes. 


\subsubsection{Adsorption isotherms and kinetics}

\subsubsection{Adsorption kinetics for adsorption of a mixture of heavy metals onto NIP}

Studying adsorption of heavy metals from real waters at their naturally occurring $\mathrm{pH}$ values is logical because those are the conditions under which treatment will occur. On the other hand, the complexity of natural waters makes the interpretation of results difficult. For this reason, further experiments examining adsorption kinetics and isotherms were conducted in deionized water at a $\mathrm{pH}$ of 4.

A $\mathrm{pH}$ of 4 was chosen because at this $\mathrm{pH}$ the heavy metal ions are present in their $\mathrm{M}^{2+}$ forms (Baes and Mesmer, 1976), eliminating the complication of heavy metal precipitation from solution or onto the NIP surface (Somasundaran, 2006). In deionized water, carbonate species will be less important and metal hydroxide species will be important. Adsorption of $\mathrm{M}(\mathrm{OH})^{+}$is favoured over adsorption of $\mathrm{M}^{2+}$ because it has a smaller effective hydrated radius, and because the functional groups on the NIP have a charge of -1 (Xiao and Thomas, 2005). At a neutral $\mathrm{pH}$, there is a mixture of different metal species present, and some metals have a greater proportion of $\mathrm{M}(\mathrm{OH})^{+}$ions present than others. Thus competition between different heavy metals is more straightforward at a lower $\mathrm{pH}$, where all metals are present as $\mathrm{M}^{2+}$. Figures showing the distribution of hydrolysis products with $\mathrm{pH}$ for each of the heavy metals studied are provided in Appendix G. At a pH of 7 or 8 (the wastewater had a $\mathrm{pH}$ of 8.01) $\mathrm{Pb}(\mathrm{OH})^{+}, \mathrm{Zn}(\mathrm{OH})_{2}, \mathrm{Cu}(\mathrm{OH})^{+}$, and $\mathrm{Cu}_{2}(\mathrm{OH})_{2}{ }^{2+}$ become significant and cannot be ignored. A pH of 4 was chosen to avoid these complications. However, there are also disadvantages to studying adsorption of heavy metals at an acidic $\mathrm{pH}$. The PKa for carboxyl functional groups is 4.5 , meaning that there will be no significant charge on the 
NIP surface at $\mathrm{pH} 4$ (Xiao and Thomas, 2004). Adsorption of NIP can also be considered an ion-exchange process between $\mathrm{H}+$ ions and heavy metal ions on the carboxylic acid functional groups, so increasing the number of $\mathrm{H}+$ ions present by decreasing the $\mathrm{pH}$ increases competition for those sites.

The kinetics of the adsorption process is an important consideration for application purposes. Practically, the NIP would not be given two weeks to reach equilibrium during full-scale application. A high degree of removal must be achieved within a reasonable time frame. One of the drawbacks to using ion-exchange for heavy metal removal is, in fact, that the rate of ion exchange can be slow. The kinetics of ion-exchange processes are typically controlled by diffusion and influenced by the degree of cross-linking, location of functional groups, charge density, and whether the polymer matrix is hydrophobic or hydrophilic. Generally, the degree of cross-linking, the nature of the polymer matrix, and the size of the polymer beads can be controlled to increase the rate of ion-exchange. NIP are very small $(217 \pm 4 \mathrm{~nm})$ in comparison to the bead size typically used for fixed-bed ion exchangers (0.3-1.2 mm), and kinetics are expected to be much faster (SenGupta, 2002).

Figure 6-5 shows the kinetics for removal of lead from deionized water at $\mathrm{pH} 4$ for a constant initial lead concentration of $500 \mathrm{ppb}$. The results shown are averages of two replicates and the error bars represent the maximum and minimum values measured. Looking at Figure 6-5 (a), the 5 day (120h) measurement appears to be an outlier, which does not follow the general trend of the data. It is possible that the samples for this point were not $\mathrm{pH}$ adjusted. 


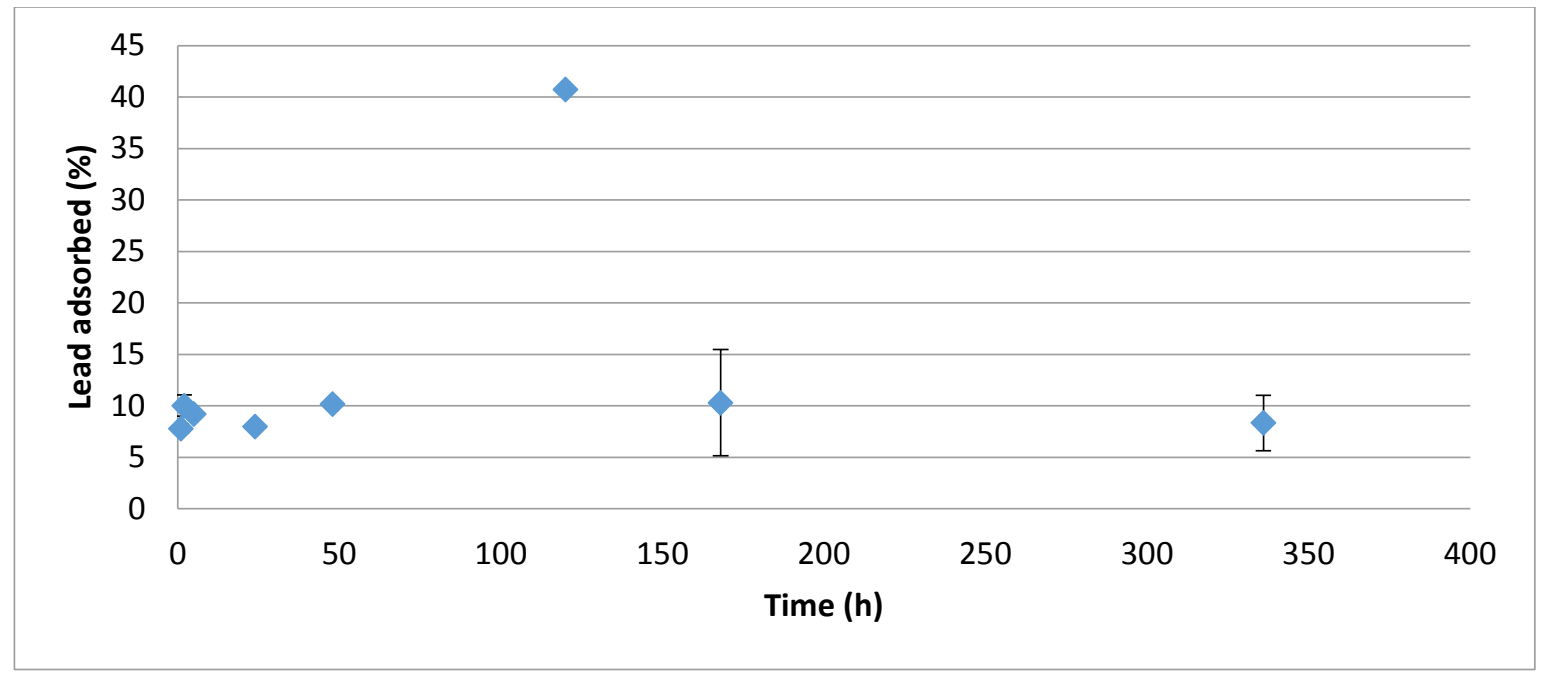

Figure 6-5: Kinetics for adsorption of lead from deionized water (pH 4)

Overall, there does not appear to be a clear increase or decrease in the percent removal of lead with time. There were some random fluctuations around an average percent removal of $9 \pm 1 \%$, but no sustained increases or decreases. The percent removal $(9 \pm 1 \%)$ was much less than that recorded for lead in river water $(82 \pm 0.2 \%)$ or wastewater $(86 \pm 0.1$ $\%$ ) even though the natural water samples were tested with a mixture of four heavy metals all present at $500 \mathrm{ppb}$. This can be explained by the low $\mathrm{pH}$. However, despite the low value for percent removal of lead, equilibrium was reached within 2 hours.

\subsubsection{Adsorption experiments for adsorption of lead onto NIP}

Isotherm studies for adsorption of lead alone (Figure 6-6) and a mixture of heavy metals (Figures 6-7 and 6-8) on NIP were also conducted at $\mathrm{pH} 4$ in deionized water. To obtain the isotherms, the mass of adsorbent was varied for a constant heavy metal concentration of $500 \mathrm{ppb}$, and the final liquid-phase concentration of the heavy metal was measured. The 
results shown are averages of two replicates and the error bars represent the maximum and minimum values. As previously mentioned, experiments were conducted at $\mathrm{pH} 4$ to simplify the system and ensure that lead was present in its $\mathrm{Pb}^{2+}$ form, but reducing the $\mathrm{pH}$ also reduces adsorption by protonating functional groups on the NIP surface and increasing competition from $\mathrm{H}+$ ions.

Figure 6-6 (a) shows the amount of free lead remaining in solution at equilibrium versus the NIP concentration. Linearized versions of the Freundlich and Langmuir models are shown in Figures 6-6 (b) and (c). The average percent absolute error was calculated and used to evaluate which model provided the best fit for the data. The average percent absolute error values were $2 \%, 17 \%$, and $87 \%$ for the models described in Figures 6-6 (a), (b), and (c), respectively. This indicates that the amount of free lead remaining in solution was best described by a linear relationship with the NIP concentration, but that the Langmuir model described the experimental results better than the Freundlich model. Additionally, the linearized Langmuir model shown in Figure 6-6 (c) has a cluster of 5 data points on the left hand side of the trend line, which heavily impact the regression. The remaining points appear to be explained well by the trend line. The Langmuir adsorption model assumes that adsorption is energetically homogeneous and occurs in a monolayer. It also assumes that adsorption and desorption from one site occurs independently of surrounding adsorption sites (Kammerer et al., 2011). This may not be the best assumption for the NIP because heavy metal ions can be involved in ion exchange reactions with neighbouring carboxyl groups. The adsorbent loading for an NIP concentration of $50 \mathrm{mg} / \mathrm{L}$ was $0.67 \pm 0.22 \mathrm{mg} / \mathrm{g}$. This can be compared to values for $50 \mathrm{mg} / \mathrm{L}$ and $500 \mathrm{ppb}$ for heavy 
metals in river water $(4.1 \pm 0.08 \mathrm{mg} / \mathrm{g})$ and wastewater $(2.83 \pm 0.002 \mathrm{mg} / \mathrm{g})$. As mentioned previously, the reduced adsorbent-phase concentration recorded for the isotherm in deionized water was probably due to the acidic $\mathrm{pH}$.

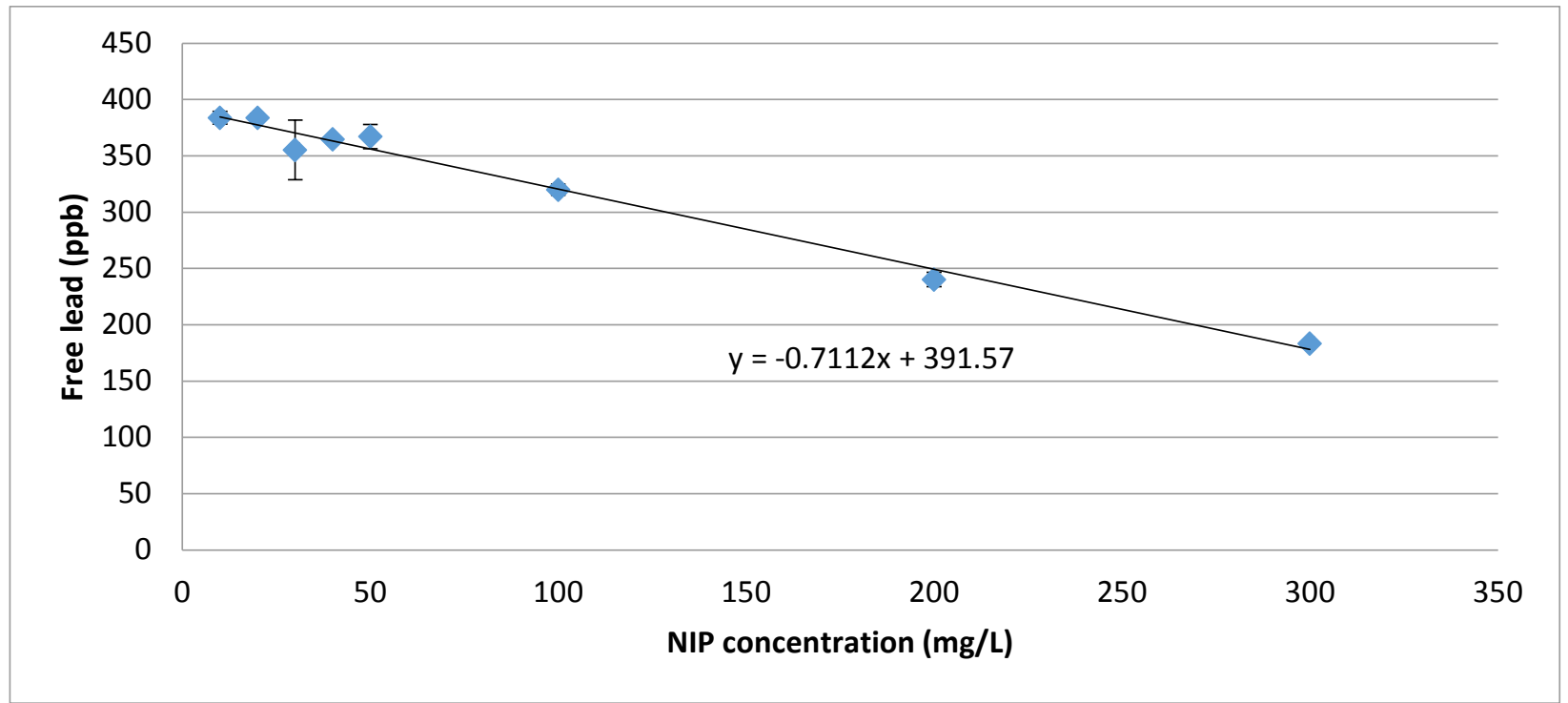

Figure 6-6 (a): Lead isotherm for adsorption onto NIP in deionized water ( $\mathrm{pH} 4$ ) 


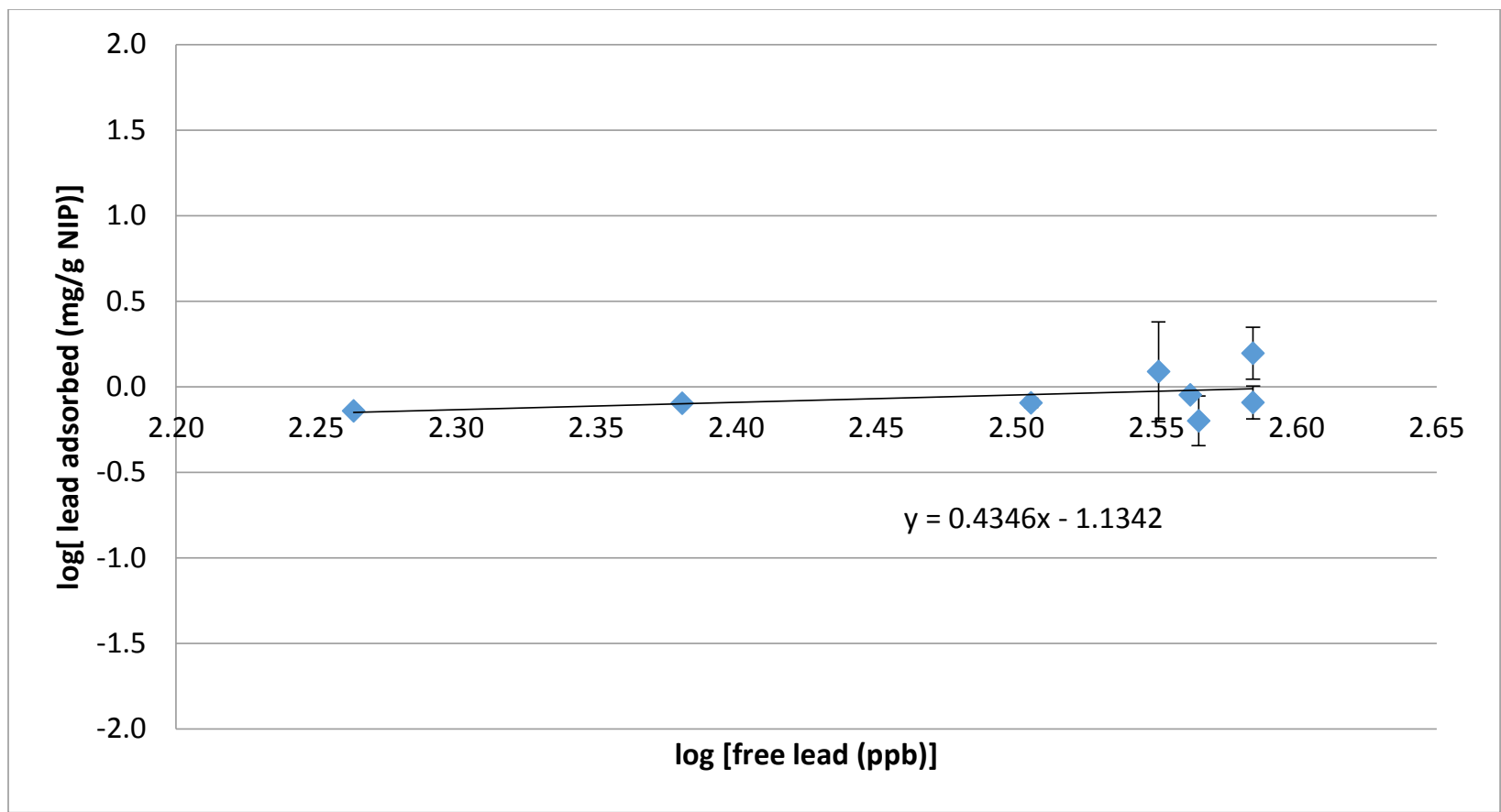

Figure 6-6 (b): Linearized Freundlich model for adsorption of lead from deionized water onto NIP (pH 4)

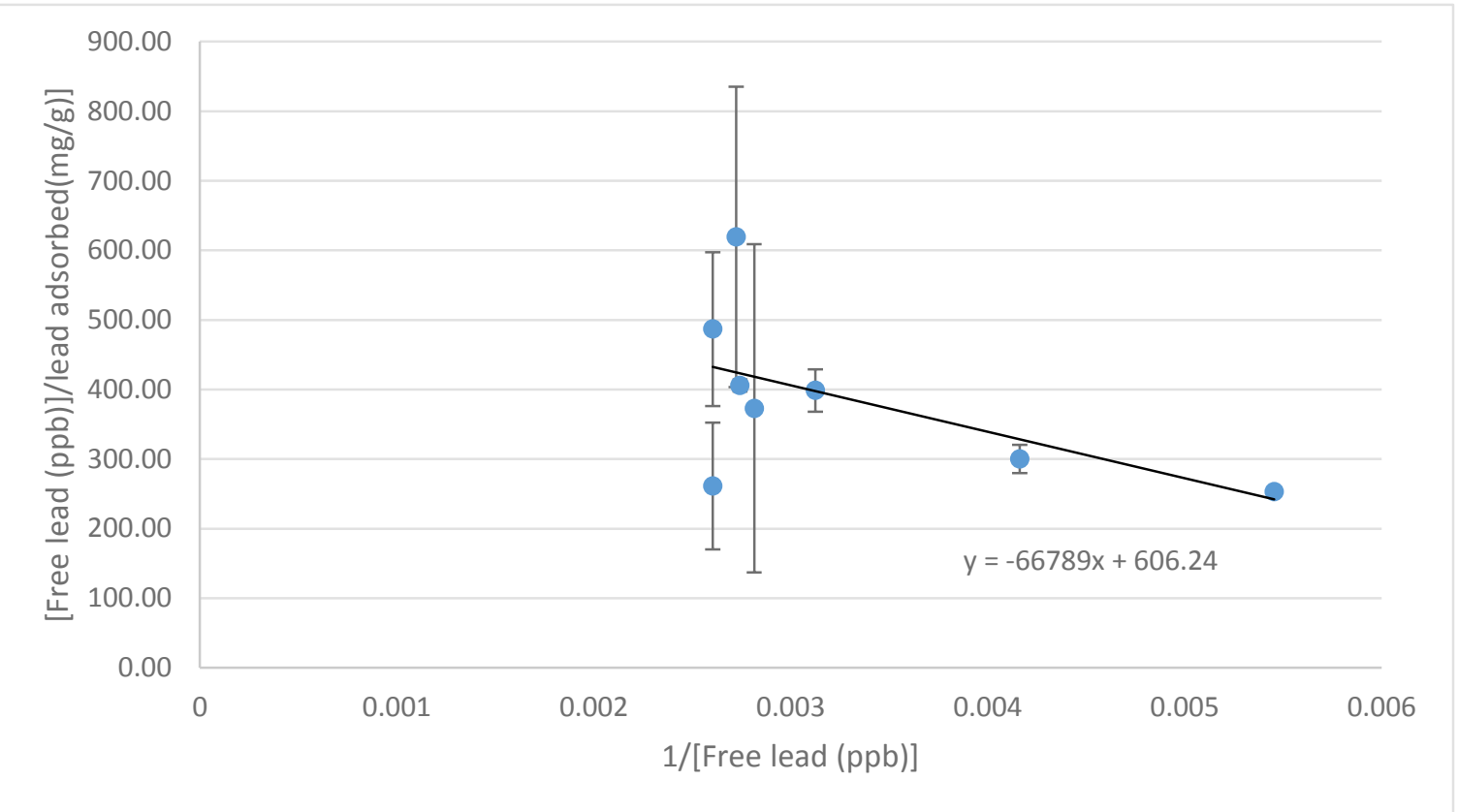


Figure 6-6 (c): Linearized Langmuir model for adsorption of lead from deionized water onto NIP ( $\mathrm{pH} 4)$

\subsubsection{Adsorption experiments for adsorption of a mixture of heavy metals onto NIP}

Isotherm data was also gathered for a mixture of $500 \mathrm{ppb}$ each of lead, copper, zinc, and nickel in deionized water at $\mathrm{pH}$ 4. Figure 6-7 (a) shows the amount of free lead remaining in solution versus the NIP concentration while Figures 6-7 (b) and (c) show the data fit to linearized forms of the Freundlich and Langmuir isotherms. The results shown are averages of two experimental replicates and the error bars represent the maximum and minimum measured values.

Similar to the results shown in Figure 6-6, the equilibrium lead concentration was linearly related to the NIP concentration with an average percent absolute error of $3 \%$. The Langmuir model did not fit the data as well as for lead alone. The average percent absolute error calculated for the linearized Langmuir model was $222 \%$. However, looking at Figure 6-7 (c), there is an outlier evident. Once the outlier was removed, the average percent absolute error was $38 \%$, while that for the Freundlich model was $40 \%$, indicating that the Langmuir model provided only a slightly better description of the data. The adsorbent loading for an NIP concentration of $50 \mathrm{mg} / \mathrm{L}$ was $0.47 \pm 0.06 \mathrm{mg} / \mathrm{g}$, which was lower than that observed for single-solute adsorption of lead under the same conditions, which was $0.67 \pm 0.22 \mathrm{mg} / \mathrm{g}$. Increased competition and adsorbent loading from other heavy metals likely caused a decrease in adsorption of lead. 


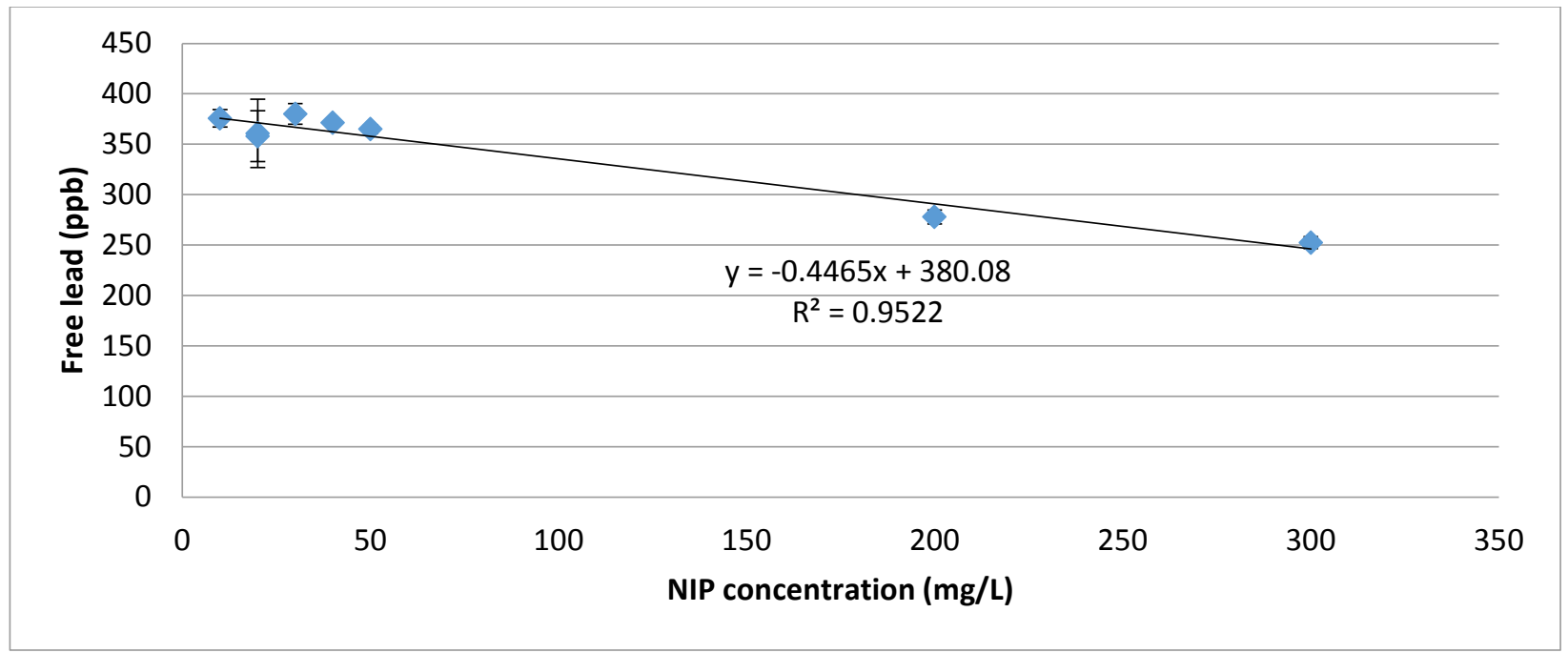

Figure 6-7 (a): Lead isotherm in deionized water for adsorption of lead onto NIP with competition from copper, zinc, and nickel ( $\mathrm{pH} 4)$

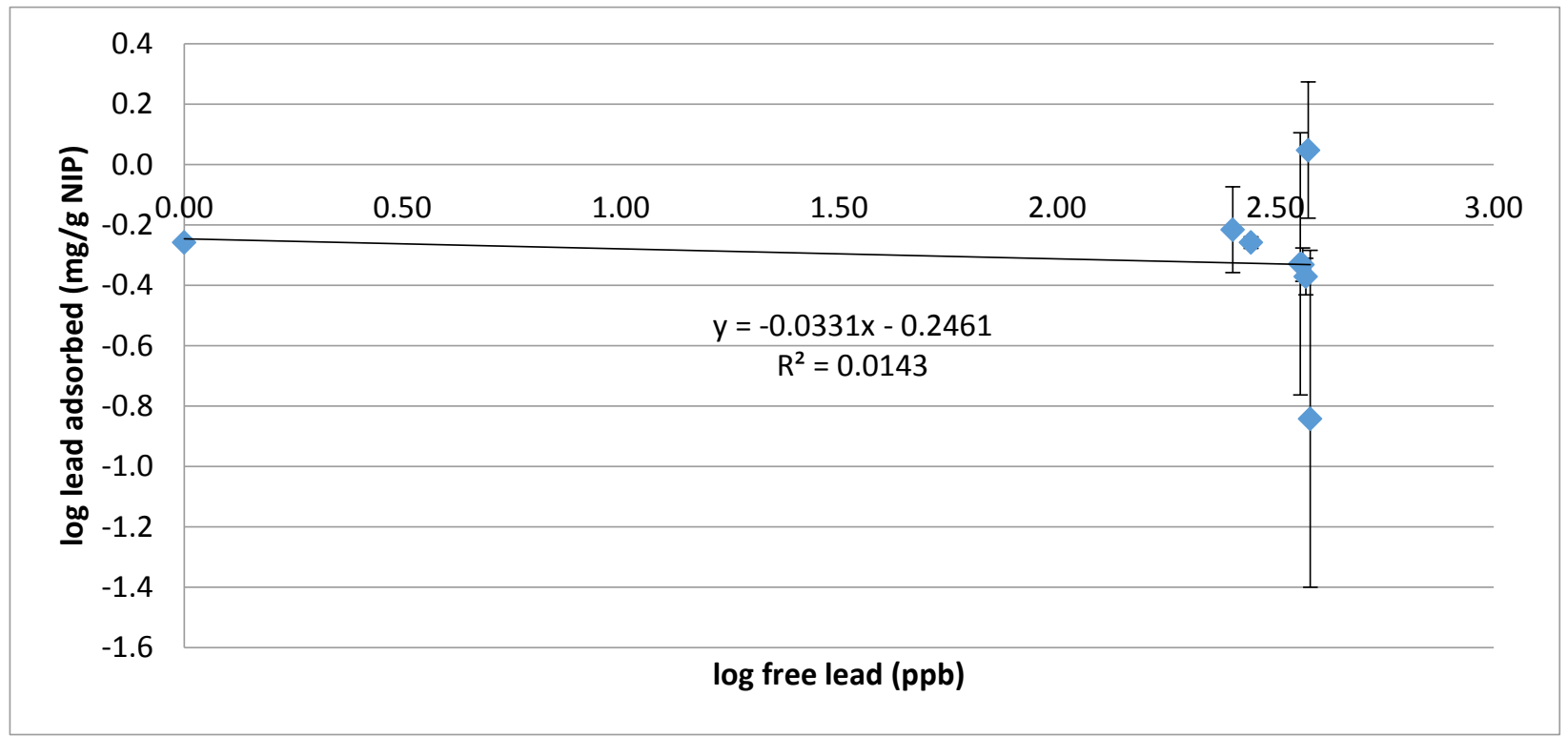

Figure 6-7 (b): Linearized Freundlich model for adsorption of lead onto NIP with competition from copper, zinc, and nickel in deionized water (pH 4) 


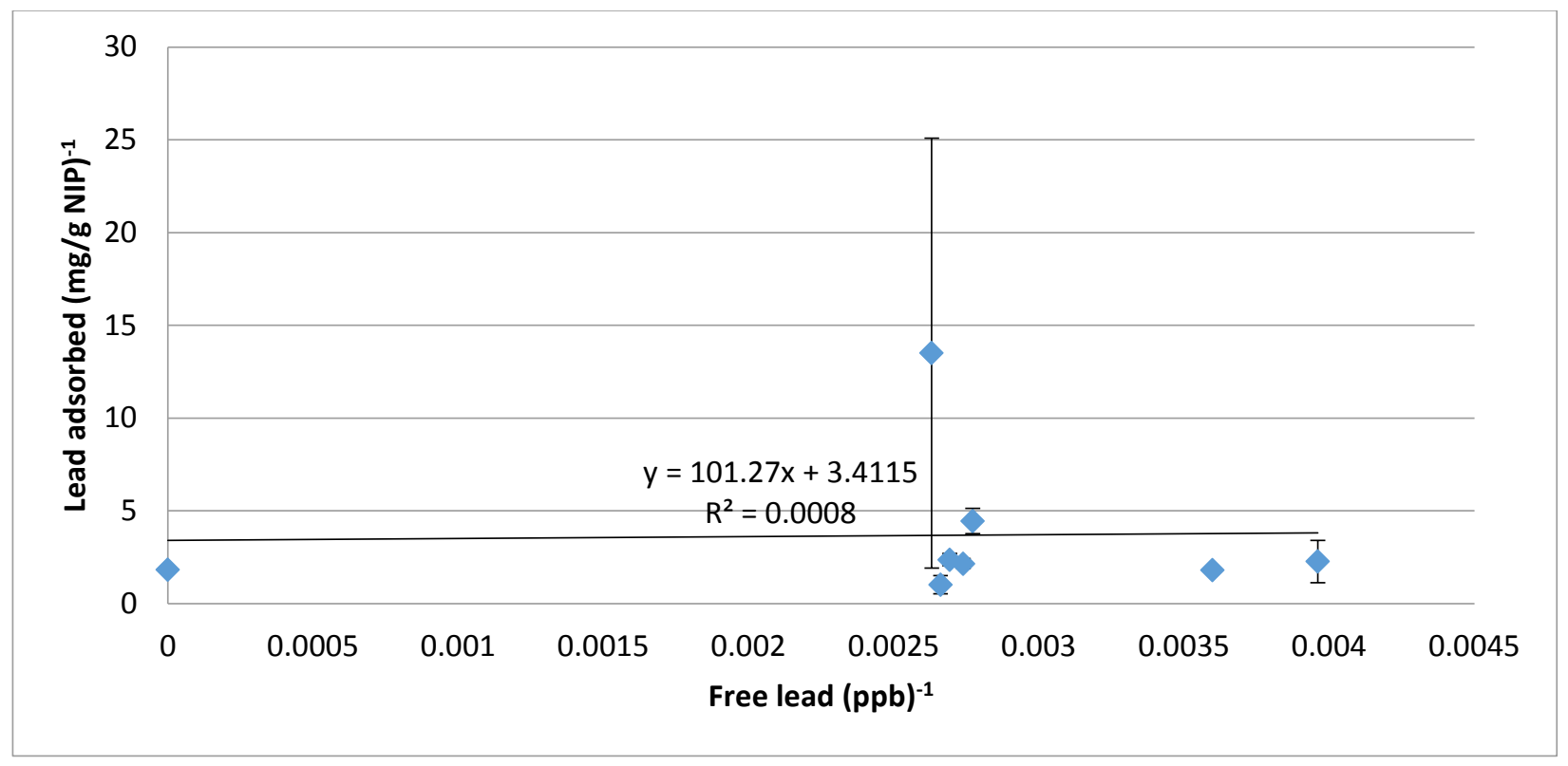

Figure 6-7 (c): Linearized Langmuir model for adsorption of lead onto NIP with competition from copper, zinc, and nickel in deionized water ( $\mathrm{pH} 4)$

Figure 6-8 (a) shows the liquid-phase heavy metal concentrations versus the NIP concentration for other heavy metals (copper, zinc, nickel) in solution, and Figure 6-8 (b) shows the percent removals versus the NIP concentration for these other heavy metal ions. It is clear from these figures that there was very little removal of other heavy metal ions from solution by the NIP particles. Isotherms are not shown because the fit for the linearized versions of the Freundlich and Langmuir isotherms was very poor. Under these conditions (at $\mathrm{pH} 4$ in deionized water), lead was preferred for removal over the other heavy metal ions. Referring to Table 6-1, this indicates that adsorption was controlled either by hydrolysis or ion-exchange. Due to the low $\mathrm{pH}$, it is unlikely that lead was present as $\mathrm{Pb}(\mathrm{OH})^{+}$or $\mathrm{Pb}(\mathrm{OH})_{2}$, indicating that adsorption likely occurred via ion-exchange. 


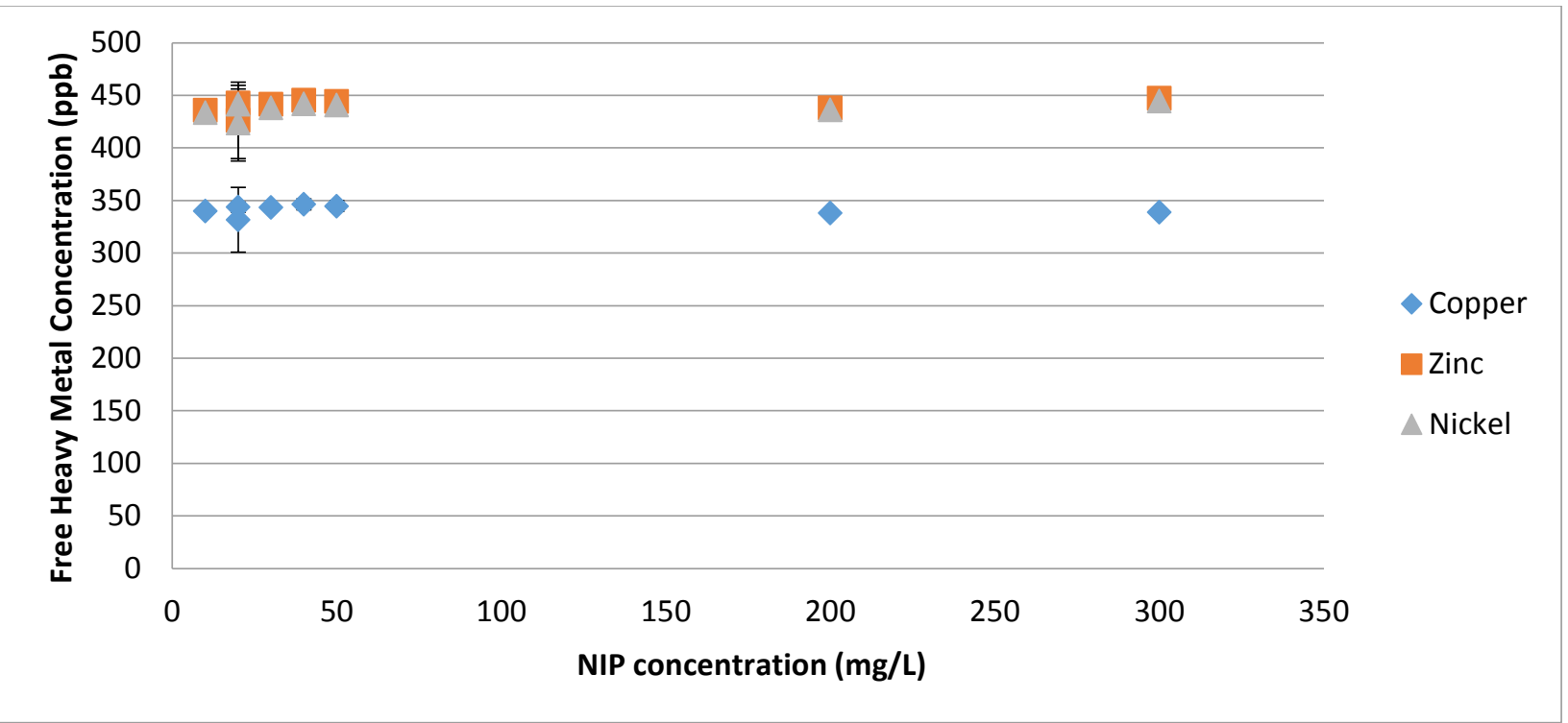

Figure 6-8 (a): Adsorption isotherms adsorption of a mixture of lead, copper, zinc, and nickel onto NIP in deionized water ( $p H 4)$

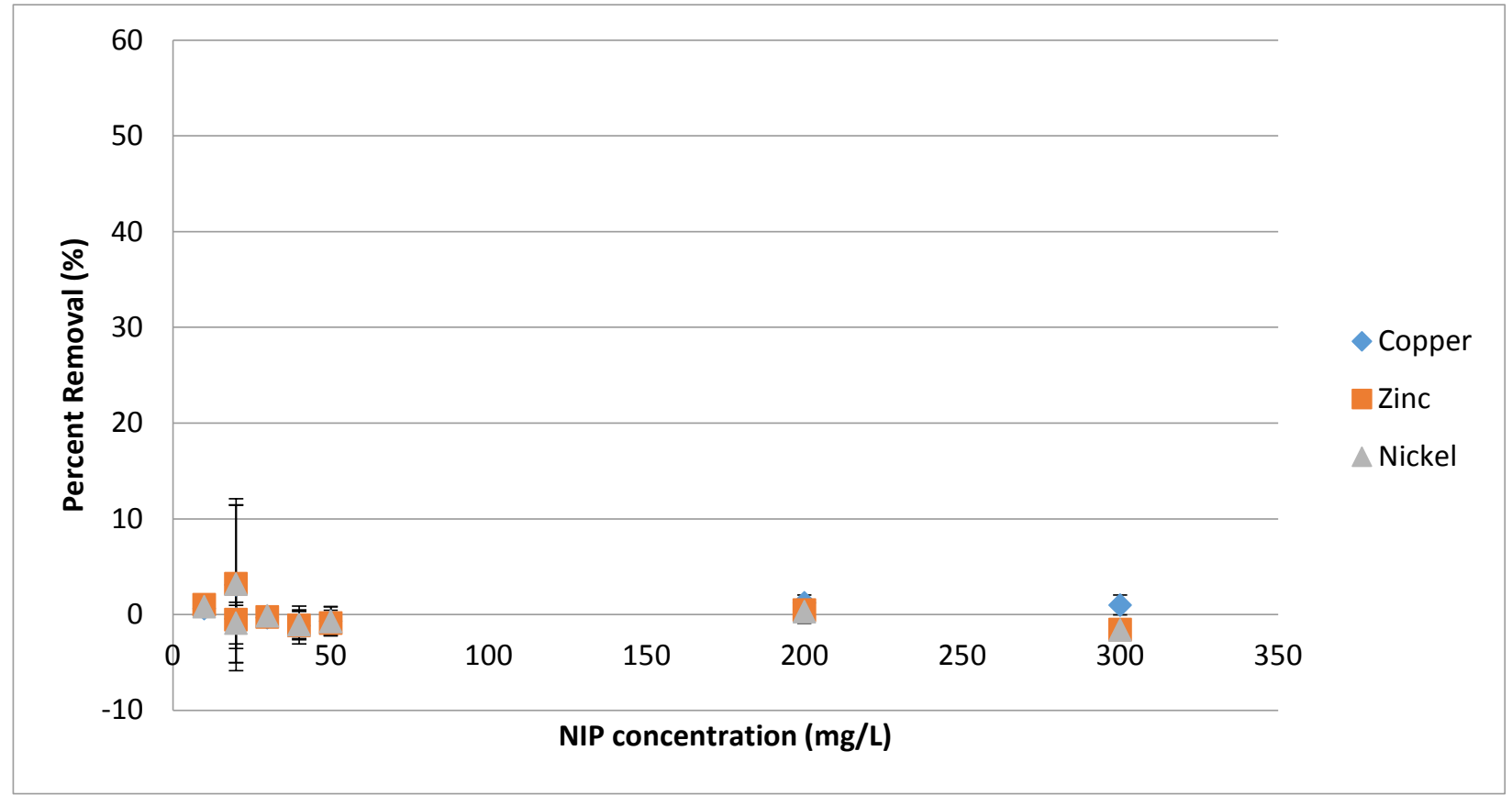

Figure 6-8 (b): Percent removal versus NIP concentration for a mixture of lead, copper, zinc, and nickel in deionized water ( $p H 4)$ 


\subsection{CONCLUSION}

The removal capacity of NIP was investigated for lead, copper, zinc, and nickel in natural and synthetic waters. NIP demonstrated excellent potential for removal of lead from natural waters, removing $82 \pm 0.2 \% 85.9 \pm 0.1 \%$ of a similar mixture from a secondary wastewater effluent. The NIP were less effective for removal of other heavy metals from natural water, which were out-competed by lead for adsorption sites. The kinetics of adsorption were fast, and equilibrium was reached in less than 2 hours, which shows the potential for fullscale application. However, competition with humic acid was shown to be a potential problem, and the addition of $20 \mathrm{mg} / \mathrm{L}$ of humic acid to deionized water, limited the removal of heavy metal ions. Isotherm tests conducted at $\mathrm{pH} 4$ in deionized water exhibited a linear relationship between the free lead remaining in solution and the adsorbent concentration at equilibrium. Isotherm studies conducted with a mixture of heavy metals at $\mathrm{pH} 4$ again showed a strong preference for lead with limited removal of other heavy metal ions. Overall, NIP demonstrated excellent potential for adsorption of lead from natural waters, but were less effective for other heavy metals, and were not effective in the presence of 20 $\mathrm{mg} / \mathrm{L}$ of humic acid.

\subsection{REFERENCES}

Al-Attas, Omar. Competitive Adsorption of Iron and Natural Organic Matter in Groundwater Using Granular Activated Carbon. Thesis. University of Ottawa, 2012. N.p.: n.p., n.d. UO Research. Web. 07 Sept. 2015. 
Alexander, C. 2006 Molecular Imprinting Science and Technology: a Survey of the Literature for the Years up to and Including 2003. Journal of Molecular Recognition, 19 (2), 106-80.

Arora, M., B. Kiran, S. Rani, A. Rani, B. Kaur, and N. Mittal. 2008 Heavy Metal Accumulation in Vegetables Irrigated with Water from Different Sources. Food Chemistry, 111 (4), 811-815.

Babel, S., and T. A. Kurniawan. 2003 Low-Cost Adsorbents for Heavy Metals Uptake from Contaminated Water: a Review. Journal of Hazardous Materials, 97 (1-3), $219-43$.

Baes, C. F., and R. E. Mesmer. 1976 The Hydrolysis of Cations. John Wiley and Sons, Toronto.

Benjamin, M., 2002. Water Chemistry. McGraw Hill, Boston.

Branger, C., W. Meouche, and A. Margaillan. 2013 Recent Advances on Ion-Imprinted Polymers. Reactive and Functional Polymers, 73 (6), 859-875.

City of Ottawa, 2006. Water Quality in Ottawa's Rivers and Streams. Prepared by the Water Environment Protection Program, Environmental Programs and Technical Support Division, Utility Services Branch, Public Works and Services, City of Ottawa.

Ebie, K., F. Li, Y. Azuma, A. Yuasa, and T. Hagishita. 2001 Pore Distribution Effect of Activated Carbon in Adsorbing Organic Micropollutants from Natural Water. Water Research, 35 (1), 167-79.

Fu, F., and Q. Wang. 2011 Removal of Heavy Metal Ions from Wastewaters: a Review. Journal of Environmental Management, 92 (3), 407-18. 
Health Canada (2014). Guidelines for Canadian Drinking Water Quality—Summary

Table. Water and Air Quality Bureau, Healthy Environments and Consumer Safety Branch, Health Canada, Ottawa, Ontario.

Jarup, L. 2003 Hazards of Heavy Metal Contamination. British Medical Bulletin, 68 (1), $167-182$.

Kammerer, J., R. Carle, and D. R. Kammerer. 2011 Adsorption and Ion Exchange: Basic Principles and their Application in Food Processing. Journal of Agricultural and Food Chemistry, 59 (1), 22-42.

Kurniawan, T. A., G. Y. S. Chan, W.-H. Lo, and S. Babel. 2006 Physico-Chemical Treatment Techniques for Wastewater Laden with Heavy Metals. Chemical Engineering Journal, 118 (1-2), 83-98.

Lai, E. P. C., Z. De Maleki, and S. Wu. 2010 Characterization of Molecularly Imprinted and Nonimprinted Polymer Submicron Particles Specifically Tailored for Removal of Trace 17b-Estradiol in Water Treatment.

Magdi, S., ed. 2013 Competitive Sorption and Transport of Heavy Metals in Soils and Geological Media. Taylor Francis \& Group, Boca Raton, Florida.

Mapanda, F., E. N. Mangwayana, J. Nyamangara, and K. E. Giller. 2005 The Effect of Long-Term Irrigation Using Wastewater on Heavy Metal Contents of Soils Under Vegetables in Harare, Zimbabwe. Agriculture, Ecosystems and Environment, 107 (2-3), 151-165.

Masqué, N. 2001 Molecularly Imprinted Polymers: New Tailor-Made Materials for Selective Solid-Phase Extraction. TrAC Trends in Analytical Chemistry, 20 (9), $477-486$. 
Pelekani, C., and V. . Snoeyink. 1999 Competitive Adsorption in Natural Water: Role of Activated Carbon Pore Size. Water Research, 33 (5), 1209-1219.

Province of Ontario, 2013. Provincial (Stream) Water Quality Monitoring Network. Prepared by Environment and Climate Change Ontario, http://www.ontario.ca/data/provincial-stream-water-quality-monitoring-network, Station ID: 18003303102.

Ryan, J., H. Pahren, and J. Lucas. 1982 Controlling Cadmium in the Human Food Chain: a Review and Rationale Based on Health Effects. Environmental Research, 28 (2), $251-302$.

SenGupta, A. K. 2002 Environmental Separation of Heavy Metals: Engineering Processes. Lewis Publishers, CRC Press, Washington, D.C.

Singh, A., R. K. Sharma, M. Agrawal, and F. M. Marshall. 2010 Health Risk Assessment of Heavy Metals via Dietary Intake of Foodstuffs from the Wastewater Irrigated Site of a Dry Tropical Area of India. Food and Chemical Toxicology, 48 (2), 611619

Singh, R., N. Gautam, and R. Gupta. 2011 Heavy Metals and Living Systems: An Overview. Indian Journal of Pharmacology, 43 (3), 246-253.

Somasundaran, P., ed. 2006 Encyclopedia of Surface and Colloid Science. Taylor Francis \& Group, Boca Raton, Florida.

Stumm, W., Morgan, J., 1981. Aquatic Chemistry: An Introduction Emphasizing Chemical Equilibria in Natural Waters, 2nd ed. Wiley-Interscience, New York. 
Toze, S. 2006 Reuse of effluent water - Benefits and risks. Agricultural Water Management, 80 (1-3 SPEC. ISS.), 147-159.

Wang, X., T. Sato, B. Xing, and S. Tao. 2005 Health Risks of Heavy Metals to the General Public in Tianjin, China via Consumption of Vegetables and Fish. Science of the Total Environment, 350 (1-3), 28-37.

Wei, S., A. Molinelli, and B. Mizaikoff. 2006 Molecularly Imprinted Micro and Nanospheres for the Selective Recognition of 17Beta-Estradiol. Biosensors \& Bioelectronics, 21 (10), 1943-51.

Xiao, B., and K. Thomas. 2005 Adsorption of Aqueous Metal Ions on Oxygen and Nitrogen Functionalized Nanoporous Activated Carbons. Langmuir, (11), 38923902.

Xiao, B., and K. M. Thomas. 2004 Competitive Adsorption of Aqueous Metal Ions on an Oxidized Nanoporous Activated Carbon. Langmuir, 20 (11), 4566-4578. 


\title{
CHAPTER 7: COMPETITIVE EFFECTS OF HUMIC ACID AND WASTEWATER ON ADSORPTION OF METHYLENE BLUE DYE BY ACTIVATED CARBON AND NON-IMPRINTED POLYMERS
}

\author{
Audrey Murray and Banu Örmeci \\ Department of Civil and Environmental Engineering, Carleton University, 1125 Colonel \\ By Drive, Ottawa ON K1S 5B6, Canada.
}

\subsection{ABSTRACT}

Natural organic matter (NOM), present in natural waters and wastewater, decreases adsorption of micropollutants, increasing treatment costs. This research investigated mechanisms of competition for non-imprinted polymers (NIP) and three activated carbon (Norit PAC 200, Darco KB-M, and Darco S-51) with humic acid and wastewater. Generally, competition occurs directly via competition for adsorption sites, or indirectly by pore-blocking, restricting access to adsorption sites. Experiments were conducted for single-solute adsorption of methylene blue dye, simultaneous adsorption with humic acid and wastewater, and pre-loading with humic acid and wastewater followed by adsorption of methylene blue dye. A decrease in adsorption capacity between single-solute adsorption and simultaneous adsorption is indicative of competition, and a further decrease for preloaded adsorbents is indicative of a pore blocking mechanism. The only decrease observed was for simultaneous adsorption with humic acid for NIP (for a $90 \%$ confidence limit). Humic acid and wastewater were found to increase adsorption for Norit PAC 200 for preloading with humic acid and simultaneous exposure to wastewater. Adsorbed humic acid 
or NOM from wastewater may have increased the negative surface charge of the activated carbon and increased adsorption, cancelling out the decreases due to competition.

\subsection{INTRODUCTION}

Micropollutants, such as pharmaceuticals and endocrine disrupting compounds, released into the environment in wastewater effluent, contaminate freshwater resources and are harmful to both human health and the environment (Gillesby and Zacharewski, 1998; Bögi et al., 2003; Segner et al., 2003; Yang et al., 2006; Helfman, 2007; Ashby et al., 1997; Bergman et al., 2013; Rochester, 2013). Micropollutants are present in wastewater at very low concentrations, typically in the order of parts per billion or parts per million. Treatment of micropollutants is challenging because wastewater effluents typically contain natural organic matter (NOM) at concentrations several orders of magnitude higher than that of the micropollutants. A significant fraction of the capacity of various treatment methods implemented to target micropollutants is consumed treating NOM instead, increasing the cost of treatment.

Molecularly imprinted polymers (MIP) are designed to specifically target a single micropollutant or group of micropollutants despite competition from other pollutants present at much higher concentrations, ensuring that resources are not consumed treating NOM instead of micropollutants. MIP are polymers formed around a template molecule. Following polymerization, the template is removed, but the MIP possess an affinity for the template molecule or molecules of similar shape and size (Masqué, 2001). Non-imprinted 
polymers (NIP) are prepared in the same way, but polymerization is performed in the absence of a template molecule and they do not specifically target a template.

MIP are able to remove micropollutants with high efficiency despite the presence of competition in natural waters (Meng et al., 2005; Zhongbo and Hu, 2008; Hajizadeh et al., 2010; Deng et al., 2009; Luo et al., 2011; Guo et al., 2011; Li et al., 2009; Yu et al., 2008; Lin et al., 2008; Krupadam et al., 2010; Le Noir, Lepeuple, et al., 2007; Le Noir, Plieva, et al., 2007; Fernández-Alvarez et al., 2009; Randhawa et al., 2007; Xie et al., 2011). This is advantageous for industrial wastewater treatment because it provides targeted treatment of one chemical or group of chemicals. However, there are a wide range of micropollutants which need to be targeted during municipal wastewater treatment, and it is not practical to create MIP for each one individually. NIP provide a non-specific alternative for municipal wastewater treatment and are capable of removing a wide range of different micropollutants (Refer to Chapters 5 and 6).

Because NIP are nonspecific, they do not have the advantage of being able to specifically target a single compound, and may experience competition from NOM in natural waters the same way that activated carbons do. Xie et al (2011) found that NOM in river water and lake water interfered with adsorption of bisphenol A less for NIP than activated carbon (Xie et al., 2011), and Murray et al (2009) found that NIP could reduce ammonia, TOC, and COD concentrations in wastewater without reduced adsorption of E2 (Murray, 2009). This suggests that NIP may possess an advantage over activated carbon in natural waters. 
However, further studies are needed as the differences in the adsorption characteristics of activated carbon and NIP are not yet understood.

NIP are synthetic polymers, and their properties can be controlled by using a different monomer, or cross-linker, or the amounts of each added (Sellergren, 1999; Masqué, 2001) as well as the temperature of polymerization (Ensing and De Boer, 1999; Yan and Row, 2006). An understanding of the differences between activated carbon and NIP, as well as the characteristics that lead to enhanced adsorption, can provide the information necessary to create NIP with better adsorption capabilities.

Adsorption processes are complex, and the differences could be due to either chemical or physical factors. The choice of monomer used in polymerization results in functional groups on the surface of the NIP capable of adsorbing micropollutants as well as competing compounds. The structure and distribution of pores is also an important parameter for micropollutant adsorption (Newcombe et al., 1997; Pelekani and Snoeyink, 1999; Pelekani and Snoeyink, 2000; Ebie et al., 2001; Pelekani and Snoeyink, 2001; Li et al., 2002; Newcombe et al., 2002; Li et al., 2003a; Matsui et al., 2003; Li et al., 2003b; Ding et al., 2008; Redding and Cannon, 2014). There are two mechanisms for competition between NOM and micropollutants: (1) direct competition for adsorption sites, and (2) pore blocking. Molecules preferentially adsorb into pores close to their molecular size (Ebie et al., 2001). Micropollutants are typically smaller than NOM and thus adsorb into smaller pores (Newcombe et al., 1997; Pelekani and Snoeyink, 1999; Pelekani and Snoeyink, 2000; Newcombe et al., 2002; Ding et al., 2008; Redding and Cannon, 2014). However, NOM 
encompasses a large range of molecular sizes, and smaller NOM molecules are able to compete directly with micropollutants while larger molecules adsorb in larger pores, blocking the entrances to small micropores (Newcombe et al., 1997; Pelekani and Snoeyink, 2001; Newcombe et al., 2002). Increasing the volume of larger pores can result in increased micropollutant adsorption because it provides more pores of the ideal size for adsorption of NOM and reduces the incidence of pore blocking (Pelekani and Snoeyink, 1999; Pelekani and Snoeyink, 2000; Redding and Cannon, 2014). Pore blocking can be detrimental to micropollutant adsorption, because, in comparison to direct competition, adsorption of relatively few competing molecules can cause a significant decrease in capacity (Pelekani and Snoeyink, 2001).

NIP are very small spherical particles with an average diameter of $217 \pm 4 \mathrm{~nm}$. In comparison, the activated carbons used in this study had median particle diameters between 25 and $45 \mu \mathrm{m}$, more than 100 times that of the NIP. As such, NIP have a large area available on their outer surface for adsorption. It is possible that this area adsorbs larger NOM molecules leaving the inner pores available for adsorption of micropollutants. This research will investigate adsorption of methylene blue dye, which is similar in size to many micropollutants, from water containing humic acid and from wastewater with both NIP and activated carbon. 


\subsection{MATERIALS AND METHODS}

\subsubsection{Adsorbents}

\subsubsection{Activated carbon}

Three powder activated carbon samples were obtained from Cabot Norit: Norit PAC 200, Darco KB-M, and Darco S-51 (Marshall, Tx). Norit PAC 200 is a coal-based activated carbon which is suited for the removal of taste, odor, and color from water. Darco KB-M is designed for removal of high molecular weight organics or dyes. Darco S-51 is designed for removal smaller molecular weight organics such as sugars, and combined with Darco KB-M, provides removal of a range of molecular weight organics. Additional information from the manufacturer is available in Appendix C.

\subsubsection{NIP Synthesis}

Functional monomer methacrylic acid (MAA) (Sigma-Aldrich; Oakville, Canada) and cross-linker ethylene glycol dimethacrylate (EGDMA) (Sigma-Aldrich; Oakville, Canada) were dissolved in a porogen with a molar ratio of $1 \mathrm{mmol}: 8 \mathrm{mmol}: 6.7 \mathrm{mmol}$ (Wei et al., 2006). The porogen was composed of $40 \mathrm{~mL}$ of 1:3 (v:v) acetone (Fisher Scientific; Ottawa, Canada), and acetonitrile (Fisher; Ottawa, Canada). 2\% (w:w) of 2isobutryonitrile (AIBN) was added as the initiator (Sigma-Aldrich; Oakville, Canada). The mixture was mixed with a vortex mixer (Fisher Scientific Vortex Mixer, USA), deoxygenated with nitrogen for five minutes, and then placed in a $60^{\circ} \mathrm{C}$ hot water bath for 24h (Isotemp 220, Fisher, USA). The resulting polymer particles were dewatered using a centrifuge (Thermo Scientific Sorval Legend $\mathrm{RT}^{+}$, Fisher Scientific) at 10,000 RPM, air dried at room temperature, and ground manually. 


\subsubsection{Adsorbent characterizations}

BET surface area, average pore size, pore volume, and mesopore volume were measured by Engineering Performance Solutions (Jacksonville, FL). Barret-Joyner-Halenda (BJA) and Quenched Solid Density Functional Theory (QSDFT) analyses were performed using a NOVA BET surface analyzer. The NOVA BET surface analyzer measures the pore volume of the adsorbent as a function of the partial pressure of nitrogen. A sample cell was submerged in liquid nitrogen to maintain a constant temperature of $77 \mathrm{~K}$. The sample cell was slowly filled with nitrogen gas, and the volume of gas was recorded for several pressure intervals to create isotherms. From the isotherm data, the BET surface area, pore volume, average pore size, and mesopore volumes were calculated. The micropore volume was then calculated by subtracting the mesopore volume from the total pore volume, and the ratio of the micropore volume to the mesopore volume was calculated by dividing these two values.

BET surface area was calculated by estimating the number of molecules of nitrogen required to cover the surface of the adsorbent in a monolayer. If the number of adsorbate molecules and the size of those molecules are known, they can be multiplied to calculate the surface area of the adsorbent (Quantachrome Corporation, 2013).

The total pore volume was calculated from the total volume of nitrogen gas adsorbed and the density of the nitrogen gas at $273 \mathrm{~K}$. The average pore size was calculated from the total pore volume using the BJH and QSDFT methods for macropores and micropores, respectively. 
The mesopore volume was calculated based on the BJH method. The BJH method was the earliest method developed to estimate the pore size distribution for activated carbons, and assumes cylindrical pores (Eduardo and Tascon, 2008). In the BJH method, isotherm data consisting of adsorbed nitrogen gas volumes versus pressures, are matched mathematically using software to those expected from a certain pore distribution (Quantachrome Corporation, 2013).

The QSDFT method, more accurately, accounts for surface roughness and heterogeneity (Quantachrome Corporation, n.d.). The QSDFT method was used to calculate the micropore volume and the BJH and QSDFT methods were combined to calculate the average pore radius. Both methods use isotherms of adsorbed nitrogen gas volumes versus pressures, which are matched using software and mathematical analysis to those expected from a certain pore distribution and shape. As the adsorbate pores are filled with nitrogen, and knowing the density of the nitrogen gas, the volume occupied by the gas can be calculated. The hysteresis during adsorption and desorption can be matched to a specific pore distribution (Quantachrome Corporation, n.d.).

\subsubsection{Humic acid}

A Suwannee River Humic Acid sample (Suwanee River Humic Acid II) was purchased from the International Humic Substances Society (St. Paul, Mn). A chemical characterization and a characterization of the functional groups in the humic acid are provided in Table 7-1 below. 
Table 7-1: Chemical Properties and Functional Groups for Suwanee River II Humic Acid

\begin{tabular}{|l|l|l|l|l|l|l|l|l|l|}
\hline \multicolumn{7}{|l|}{ Chemical Characterization (International Humic Substances Society, 2015a) } \\
\hline C (\%) & $\mathrm{H}(\%)$ & $\mathrm{O}(\%)$ & $\begin{array}{l}\mathrm{N} \\
(\%)\end{array}$ & $\begin{array}{l}\mathrm{S} \\
(\%)\end{array}$ & $\mathrm{P}(\%)$ & & & & \\
\hline 52.63 & 4.28 & 42.04 & 1.17 & 0.54 & 0.013 & & & & \\
\hline Functional Groups & International Humic Substances Society, 2015b) ** & \\
\hline Carboxyl & Phenolic & $\mathrm{Q}_{1}$ & $\begin{array}{l}\text { Log } \\
\mathrm{k}_{1}\end{array}$ & $\mathrm{n}_{1}$ & $\mathrm{Q}_{2}$ & $\begin{array}{l}\text { Log } \\
\mathrm{k}_{2}\end{array}$ & $\mathrm{n}_{2}$ & $\mathrm{~N}$ & $\mathrm{RMSE}$ \\
\hline 9.13 & 3.72 & 9.74 & 4.35 & 3.30 & 4.48 & 10.44 & 1.73 & 171 & 0.0815 \\
\hline
\end{tabular}

\footnotetext{
** The carboxyl functional groups are represented by the charge density (meq/gC) at $\mathrm{pH} 8$. The phenolic functional groups are measured by the change in charge density between $\mathrm{pH} 8$ and $10 . \mathrm{Q}_{1}$ and $\mathrm{Q}_{2}$ are the maximum charge densities of the carboxyl and phenolic functional groups, respectively, and $\log \mathrm{k}_{1}$ and $\log \mathrm{k}_{2}$ are the mean $\log \mathrm{k}$ values for binding of protons. The empirical parameters, $\mathrm{n}_{1}$ and $\mathrm{n}_{2}$, describe the width of the variability of the Log $\mathrm{k}$ values. $\mathrm{N}$ is the number of points used for the titration, and RMSE is the root of the mean square error for fitting the data to a Langmuir-Freundlich model.
}

\subsubsection{Wastewater}

Secondary wastewater effluent was collected from the Robert O. Pickard Environmental Centre in Ottawa, Ontario. It was stored in the refrigerator and then left at room temperature overnight prior to use.

\subsubsection{Single-solute adsorption}

Adsorption isotherm tests were conducted to evaluate removal of methylene blue (MB) dye from deionized water using NIP, Norit PAC 200, Darco KB-M, and Darco S-51. 150 $\mathrm{mL}$ amber glass bottles were filled with $50 \mathrm{~mL}$ of deionized water spiked with 10,12, 14, 16,18 or $20 \mathrm{ppm}$ of MB dye. The concentrations of MB dye used were much larger than those of micropollutants present in natural waters; however, the purpose of the experiments was to determine how micropollutants enter the pores and access adsorption sites on NIP 
versus powdered activated carbon. Therefore, the size of the MB molecules was the main concern. $50 \mathrm{~g} / \mathrm{L}$ solutions were prepared for the NIP and three activated carbons, and sonicated (Vibracell Sonics, Sonics and Materials Inc, Newtown Conneticut) for 15 minutes to disperse the particles. $1 \mathrm{~mL}$ of the NIP solution or $0.1 \mathrm{~mL}$ of each activated carbon solution was then added to each sample bottle to obtain final concentrations of 0.1 $\mathrm{g} / \mathrm{L}$ for the activated carbons and $0.5 \mathrm{~g} / \mathrm{L}$ for the NIP. The samples were mixed on a shaker table (New Brunswick Scientific Excella E1 Platform Shaker, New Brunswick Scientific Inc) for two weeks. They were then centrifuged (Thermo Scientific Sorval Legend RT+, Fisher Scientific) for 1 hour at a speed of 10,000 rpm (670, 800g). Following centrifugation, the centrate was poured into $15 \mathrm{~mL}$ centrifuge bottles and analyzed with a UV-visible spectrophotometer (Varian Model Cary 100Bio; Victoria, Australia) using a scan from 200 to $800 \mathrm{~nm}$. A peak for MB was identified at $663 \mathrm{~nm}$, which did not experience any interference from humic acid or wastewater, and was used for data analysis. PH was not monitored.

\subsubsection{Simultaneous loading}

Isotherm experiments were conducted using $\mathrm{MB}$ in a $20 \mathrm{mg} / \mathrm{L}$ humic acid solution and secondary wastewater effluent. Samples were adjusted to $\mathrm{pH} 7$ using sodium hydroxide and hydrochloric acid prior to addition of MB. The procedure outlined above for singlesolute adsorption (Section 7.3.5) was then followed using NIP and Norit PAC 200 as adsorbents. Control samples were prepared without adsorbents using the same procedure (including centrifuging) and used to generate calibration curves. The $\mathrm{pH}$ was not monitored during or following adsorption experiments. PH was not monitored. 


\subsubsection{Pre-loading}

Isotherm experiments were also conducted using NIP and Norit PAC 200 that were first exposed to humic acid or wastewater solutions and then subsequently exposed to MB. 50 $\mathrm{mL}$ of the $20 \mathrm{mg} / \mathrm{L}$ humic acid solution or secondary wastewater effluent were added to $150 \mathrm{~mL}$ amber glass bottles. NIP or Norit PAC 200 were added to obtain final concentrations of $0.5 \mathrm{~g} / \mathrm{L}$ or $0.1 \mathrm{~g} / \mathrm{L}$, respectively. The bottles were placed on a shaker table (New Brunswick Scientific Excella E1 Platform Shaker; New Brunswick Scientific Inc.) for 24 hours. Following the 24 hour adsorption period, the samples were centrifuged (Thermo Scientific Sorval Legend RT+; Fisher Scientific) in $50 \mathrm{~mL}$ centrifuge tubes for 1

hour at $10,000 \mathrm{rpm}(670,800 \mathrm{~g}) .50 \mathrm{~mL}$ of dionized water with methylene blue ranging from 10-20 ppm was added to the centrifuge tubes and they were shaken to disperse the particles. The solution was then poured into $150 \mathrm{~mL}$ amber glass bottles, shaken on a platform shaker for two weeks, and centrifuged, and the centrate was analyzed using UV visible spectrophotometry. PH was not monitored.

\subsubsection{Statistical analysis}

To determine whether or not there was a competitive effect from humic acid or wastewater during either pre-loading or simultaneous adsorption, comparisons between sets of data for different sample conditions were necessary. Since the MB concentration was also varied for each data set, a t-test using a paired comparison of means was used. First, the differences between the average adsorption capacities for the two conditions being compared were calculated for each MB concentration. For example, to investigate the 
effect of competition from wastewater, the average adsorption capacity in wastewater for each MB concentration (10ppm-20ppm) was subtracted from the corresponding adsorption capacity in deionized water. The average and variance of these differences were calculated, and used to calculate the corresponding t-statistics and estimated probabilities for rejecting the null hypothesis. The null hypothesis was that there was no difference in adsorption capacity between the two sample groups. If the $\mathrm{P}$ value was greater than 0.1 , which corresponds to a $90 \%$ confidence limit, the difference was considered insignificant. If it was less than 0.1 , the difference was considered significant. The percent difference between the samples was calculated similarly using a paired t-test with a $90 \%$ confidence interval. The percent difference was calculated by dividing the calculated difference in adsorption capacities for the two samples by the average of the two samples and multiplying by 100 .

\subsubsection{Zeta potential}

\subsubsection{Sample preparation}

\subsection{Adsorbents in water}

$5 \mathrm{mg}$ of Norit PAC 200 or $25 \mathrm{mg}$ of NIP were weighed and added to $50 \mathrm{~mL}$ centrifuge tubes containing $50 \mathrm{~mL}$ each of deionized water. The solutions were sonicated (Vibracell Sonics, Sonics and Materials Inc., Newtown Connecticut) to disperse the particles.

\subsection{Adsorbents in humic acid}

$200 \mathrm{~mL}$ of $20 \mathrm{mg} / \mathrm{L}$ humic acid solution were prepared by adding $4 \mathrm{mg}$ of Suwanee River Humic Acid to $200 \mathrm{~mL}$ of deionized water. This solution was also used for pre-loading of 
adsorbents with humic acid as outlined in Section 7.3.9.1.3, below. $50 \mathrm{~mL}$ of this solution was added to each of two $50 \mathrm{~mL}$ centrifuge tubes. $5 \mathrm{mg}$ of Norit PAC 200 or $25 \mathrm{mg}$ of NIP were weighed and added to each tube. The solutions were sonicated for 15 minutes each to disperse the particles.

\subsection{Pre-loading of adsorbents with humic acid}

The procedure outlined in Section 7.3.9.1.2, above, was followed to create solutions with PAC 200 and NIP in $20 \mathrm{mg} / \mathrm{L}$ of humic acid. The centrifuge tubes were placed on a shaker table (New Brunswick Scientific Inc) for 24 hours. They were then centrifuged (Thermo Scientific Sorval Legend $\mathrm{RT}^{+}$, Fisher Scientific) for 1 hour at a speed of 10,000 rpm $(670,800 \mathrm{~g})$. Following centrifugation, the centrate was poured out and $50 \mathrm{~mL}$ of deionized water was added to each centrifuge tube. The solutions were sonicated for 15 minutes each to disperse the particles in the deionized water.

\subsection{Zeta potential measurements and instrument}

A Zeta Meter 4.0 (Zeta-Meter Inc, Staunton, Virginia) was used for zeta potential measurements. The Zeta Meter uses backlighting to increase the visibility of small particles that would usually require high magnification. The magnification provided by the objectives and eyepieces was 80x (Zeta Meter Inc, 2010). First, the electrophoresis cell was filled with the sample. Then the Zeta Meter was turned on. The electrophoresis cell was placed on the cell holder and the electrodes were connected. The specific conductance of the sample was measured and set with the Zeta Meter. A voltage of $300 \mathrm{~V}$ was applied. The time for a particle to travel between two tracking lines was measured by pressing the 
track key as the particle reached the first tracking line and releasing it as the particle reached the second tracking line. This was repeated for 20 particles. The instrument automatically calculated the average zeta potential and standard deviation. The electrophoresis cell was rinsed well with tap water followed by the next sample between samples.

\subsection{RESULTS AND DISCUSSION}

\subsubsection{Adsorbent characterization}

NIP and three activated carbons: Norit Darco KB-M, Norit PAC 200, and Norit Darco S51, were studied. The Brunauer-Emmett-Teller (BET) surface area, average pore size, total pore volume, micropore volume, mesopore volume, and ratio of micropore volume to mesopore volume for each adsorbent are provided in Table 7-2. In general, the NIP had the smallest BET surface area of the four adsorbents studied, followed by Darco S-51, PAC 200, and Darco KB-M. The surface area of the NIP was 176 times smaller than that of the activated carbon with the smallest surface area (Darco S-51). The NIP also had the smallest ratio of micropore volume to mesopore volume $(0.12)$, meaning that most of their pore volume was contained in larger rather than smaller pores. In contrast, PAC 200, had the highest ratio of micropore volume to mesopore volume (2.81). Generally, a higher surface area enhances adsorption, and a higher micropore volume is favorable for adsorption of micropollutants because micropollutants preferentially adsorb in smaller pores (Newcombe et al., 1997; Pelekani and Snoeyink, 1999; Pelekani and Snoeyink, 2000; Newcombe et al., 2002; Ding et al., 2008; Redding and Cannon, 2014). 
Table 7-2: Adsorbent Characterization

\begin{tabular}{|c|c|c|c|c|c|c|}
\hline & $\begin{array}{l}\text { BET } \\
\text { Surface } \\
\text { Area } \\
\left(\mathrm{m}^{2} / \mathrm{g}\right)\end{array}$ & $\begin{array}{l}\text { Pore Size } \\
(\AA)\end{array}$ & $\begin{array}{l}\text { Pore } \\
\text { Volume } \\
\left(\mathrm{cm}^{3} / \mathrm{g}\right)\end{array}$ & $\begin{array}{l}\text { Mesopore } \\
\text { volume } \\
\left(\mathrm{cm}^{3} / \mathrm{g}\right)\end{array}$ & $\begin{array}{l}\text { Micropore } \\
\text { volume } \\
\left(\mathrm{cm}^{3} / \mathrm{g}\right)\end{array}$ & $\begin{array}{l}\text { Micropore } \\
\text { volume/Mesopore } \\
\text { volume }\end{array}$ \\
\hline NIP & 3.4 & 73.06 & 0.0125 & 0.0112 & 0.0013 & 0.12 \\
\hline $\begin{array}{l}\text { Darco } \\
\text { KB-M }\end{array}$ & 992.7 & 20.12 & 0.9989 & 0.5872 & 0.4117 & 0.70 \\
\hline $\begin{array}{l}\text { PAC } \\
200\end{array}$ & 961.8 & 11.44 & 0.5502 & 0.1421 & 0.4081 & 2.87 \\
\hline $\begin{array}{l}\text { Darco } \\
\text { S-51 }\end{array}$ & 599.5 & 25.5 & 0.7643 & 0.5041 & 0.2602 & 0.52 \\
\hline
\end{tabular}

\subsubsection{Single-solute adsorption}

Methylene blue (MB) was used as a model compound for organic micropollutants. MB is similar in size to many organic micropollutants, and is able to penetrate into the same pores and occupy the same adsorption sites. Pelekani et al (2000) studied competitive adsorption between $\mathrm{MB}$ and atrazine and found that $\mathrm{MB}$ was able to compete directly for adsorption sites with atrazine. $\mathrm{MB}$ is a good model compound for experiments because it can be measured easily using UV-vis absorbance. MB was meant to represent micropollutants only in terms of size. The purpose of these experiments were to investigate the effects of NOM on adsorption of micropollutants in terms of access to porous networks, particularly investigating the effects of molecular size.

For each adsorbent, the adsorbent concentration was kept constant while the MB concentration was varied between 10-20 ppm. Figure 7-1, below, provides a plot of the 
adsorption capacity ( $\mathrm{mg}$ adsorbed/mg adsorbent) versus the initial concentration of MB. Linear trends were observed for all adsorbents. The initial concentration of MB was plotted on the $\mathrm{x}$-axis rather than the equilibrium concentration because there was no visible trend when the loading was plotted against the equilibrium concentration. Typical equilibrium concentrations of MB ranged from $0.4 \mathrm{ppm}$ to $16 \mathrm{ppm}$, depending on the adsorbent and the initial concentration of MB. The percent of MB dye removed remained constant for each of the four adsorbents as the initial concentration was increased, leading to a linear increase in the adsorption capacity with increasing concentration. The adsorption capacities plotted represent the average of three experimental replicates and the error bars represent the standard deviation. For PAC 200 and Darco KB-M, the error bars were large. Visible differences in colour between experimental replicates were observable for these two activated carbons. Every attempt was made to ensure that the activated carbon and NIP solutions were as homogeneous as possible. They were sonicated for a full 15 minutes to disperse the particles. Considering that the same procedure was also followed for Darco S-51 and the NIP, and they showed less variability between replicates, the variation is likely due to heterogeneity in the activated carbons themselves. Activated carbon samples are heterogeneous in nature, and this appears to be reflected in the results.

The kinetics of removal have the potential to effect the final amount adsorbed, and could, potentially be effected by the particle size of the powdered activated carbons. The NIP had an average particle diameter of approximately $300 \mathrm{~nm}$, while the PACs had larger average particle sizes. Darco KB-M had the smallest $\mathrm{D}_{50}$ of $25 \mu \mathrm{m}$, followed by Darco S-51 and PAC 200. Darco S-51 had a $\mathrm{D}_{50}$ of $30-42 \mu \mathrm{m}$, while PAC 200 had a minimum of $50 \%$ of 
particles smaller than $45 \mu \mathrm{m}$. Therefore, the particle sizes of the PAC samples were approximately 100 times larger than those of the NIP. Due to the differences of particle sizes, the kinetics of adsorption was expected to be much faster for the NIP than the PACs. Therefore, an adsorption time of 2 weeks was allowed to provide ample time for all adsorbents to reach equilibrium.

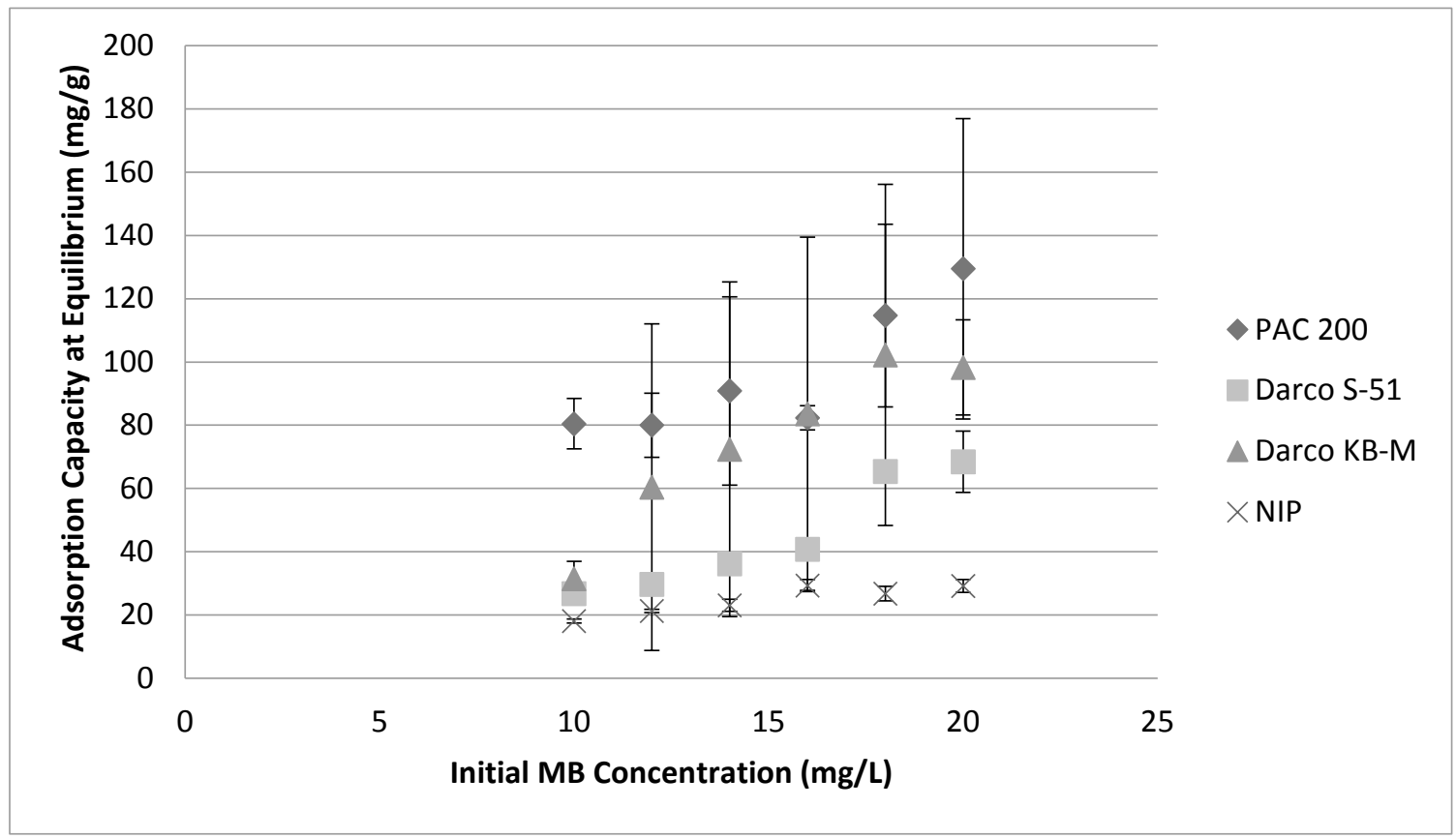

Figure 7-1: Adsorption capacities of PAC 200, Darco S-51, Darco KB-M, and NIP for varying initial $M B$ concentrations in deionized water

Referring to Figure 7-1, PAC 200 adsorbed the greatest mass of MB per mass of adsorbent, followed by Darco KB-M, Darco S-51, and finally NIP. Theoretically, the adsorbent with the greatest surface area should have the greatest adsorption capacity, but the distribution of pore sizes is also important. In fact, Wang et al (2004) found that surface area and pore volume had little effect on MB adsorption by activated carbon, which was instead 
controlled by the pore size distribution and chemical characteristics (Wang et al., 2005). The BET surface area followed the order: Darco KB-M>PAC 200> Darco S-51> NIP. On the other hand, the average pore sizes increased in order: PAC $200<$ Darco KB-M $<$ Darco S-51< NIP. Pelekani et al (2000) found that adsorption of MB correlated well with micropore sizes close to $7.5 \AA$, predicting increased adsorption for decreasing pore size. The observed performance order, shown in Figure 7-1, followed the order predicted by the pore sizes, with increasing adsorption capacities correlating well with decreasing pore size. Trendlines were not shown in Figure 7-1 for clarity. However, the slope of the line fitted to the data for Darco KB-M $(6.7 \mathrm{~L} / \mathrm{g})$ was steeper than that for PAC $200(4.9 \mathrm{~L} / \mathrm{g})$, indicating that Darco KB-M may possess a greater adsorption capacity than PAC 200 for high MB loadings. This may occur once all the micropores of ideal size for MB adsorption on PAC 200 have been occupied and further adsorption sites on PAC 200 are no longer more energetically favorable than those on Darco KB-M. Smaller pores allow the adsorbate to interact with the adsorbent three dimensionally, and overlapping potentials from the walls of the pores results in stronger binding between the adsorbate and adsorbent (Pelekani and Snoeyink, 2000).

Darco S-51 exhibited a lower adsorption capacity for MB than either PAC 200 or Darco KB-M. This was expected because it had both a lower BET surface area and a larger average pore size than either of the other two activated carbons. Surprisingly, the NIP, which possessed a BET surface area 176 times smaller than that of Darco S-51, had an adsorption capacity of $18 \pm 0.7 \mathrm{mg} / \mathrm{mg}$ for an initial MB concentration of $10 \mathrm{ppm}$ whereas the Darco S-51 had an adsorption capacity of $27 \pm 1 \mathrm{mg} / \mathrm{mg}$. The range of concentrations 
is always important for adsorption experiments. The NIP concentration used to treat 10-20 ppm MB was higher than that of the activated carbons $(0.5 \mathrm{~g} / \mathrm{L}$ as compared to $0.1 \mathrm{~g} / \mathrm{L})$, and it is clear from Figure 7-1, that the slope of the line of best fit was less steep. Increasing MB concentrations further would lead to greater increases in adsorption for the three activated carbons than it would for the NIP. Therefore, it is expected that at a higher concentration of MB, the difference in performance between Darco S-51 and NIP would be greater.

This is important if NIP is to be used as an adsorbent for water and wastewater treatment. The NIP particles were only $217 \pm 4 \mathrm{~nm}$ in diameter. They have a high surface area on the surface of the particles due to their small size, but it is still not enough to compete with the large surface area possessed by the activated carbons due to their internal porous structure. As provided in Table 7-2, the NIP have a surface area of $3.4 \mathrm{~m}^{2} / \mathrm{g}$ while the activated carbons had surface areas of 992.7, 961.8, and $599.5 \mathrm{~m}^{2} / \mathrm{g}$ for Darco KB-M, PAC 200, and Darco S-51, respectively. The disadvantage posed by the lower surface area is apparent in Figure 7-1, where it is clear that all three of the activated carbons have a higher adsorption capacity than the NIP. The large area on the outer surface of the NIP alone does not allow them to compete with activated carbon; they also require a greater pore volume to further increase their surface area and adsorption capacity. The solvent in which the NIP are created is called a porogen, and the chemistry of the porogen as well as the amount used can control the pore morphology and volume (Cormack and Elorza, 2004). Creating NIP particles with a greater specific surface area, smaller average pore size, and greater pore volume, should increase adsorption. 


\subsubsection{Competitive adsorption with humic acid}

Natural organic matter (NOM), present in natural waters and wastewater, can reduce the adsorption capacity of activated carbons by one or two orders of magnitude, and can also decrease the rate of adsorption (Pelekani and Snoeyink, 1999). Humic acid represents one fraction of the NOM present in natural waters, typically the larger fraction (Newcombe, 1994). The humic acid used in this study was Suwannee River Humic Acid provided by the International Humic Substances Society. This humic acid was chosen because it has been widely characterized. Several researchers have measured the average molecular weight of Suwannee River Humic Acid by number and by mass, and the averages of these recorded values are $1716 \pm 478 \mathrm{~g} /$ mole (by number) and $3303 \pm 904 \mathrm{~g} / \mathrm{mole}$ (by mass). Refer to Table 7-3, below, for a summary of the individual studies. There are wide distributions in the data measured by different researchers using different measurement methods, but this is to be expected from a compilation of measurements from a variety of sources. The humic acid itself also contains a wide range of molecular weights.

Table 7-3: Molecular Weight of Suwanee River Humic Acid Adapted from Perdue and Ritchie (2003) (Perdue and Ritchie, 2003)

\begin{tabular}{l|l|l|l|l|}
\hline & Year & Method ** & $\begin{array}{l}\text { Number } \\
\text { Average }\end{array}$ & $\begin{array}{l}\text { Weight } \\
\text { Average }\end{array}$ \\
$\begin{array}{l}\text { Btudy Author } \\
\text { Benedetti, Ranville, Ponthieu, }\end{array}$ & 2002 & FFF & 1320 & 2090 \\
\hline $\begin{array}{l}\text { Pinheiro } \\
\text { Dycus, Healy, Stearman, and }\end{array}$ & 1995 & FFF & 2247 & 3157 \\
$\begin{array}{l}\text { Wells } \\
\begin{array}{l}\text { Croue, Violleau, Bodaire, and } \\
\text { Legube }\end{array}\end{array}$ & 1999 & SEC & & 2600 \\
$\begin{array}{l}\text { Westerhoff, Aiken, Amy, and } \\
\text { Debroux }\end{array}$ & 1999 & SEC & & 3320 \\
\hline
\end{tabular}




\begin{tabular}{l|l|l|l|l|}
$\begin{array}{l}\text { Reid, Wilkinson, Tipping, and } \\
\text { Jones }\end{array}$ & 1990 & UV-UCGN & & 4260 \\
\hline Beckett, Jue, and Giddings & 1987 & FFF & 1580 & 4390 \\
\hline & & Average & 1716 & 3303 \\
\hline ** Flow Field-Flow Fractionation (FFF), Size Exclusion Chromatography (SEC), UV Scanning Ultracentrifugation (UV-UCGN) \\
\hline
\end{tabular}

The humic acid concentration used was higher than that typically found in surface waters, but it was a concentration that was expected to have an effect on adsorption. Also, as previously mentioned humic acid is not necessarily representative of the NOM in surface waters. However, it was very important for this study to have some idea of the molecular weight of the NOM. Although the molecular weight data for Suwanee River Humic acid varied, it provided an estimate of the molecular weight. Alternatively, surface water samples could have been taken and the sizes of NOM contained therein analyzed, perhaps using size exclusion liquid chromatography with organic carbon detection. However, the molecular sizes of NOM contained in surface waters vary considerably, and the NOM contained in surface waters is heterogeneous, so this would not necessarily have provided more information about the size of molecules competing with MB.

The Suwannee River Humic Acid was combined with MB in aqueous solution to investigate the competitive effects of humic acid on MB adsorption for activated carbon and NIP. PAC 200, the best-performing activated carbon based on single-solute experiments, was studied alongside NIP for competitive experiments. PAC 200 had the smallest average pore size (11.44 $\AA$ ) of all the adsorbents studied and the highest ratio of micropore volume to mesopore volume (2.87). NIP, on the other hand, had the largest 
average pore size $(73.06 \AA)$, and lowest micropore volume to mesopore volume ratio (0.116).

Figures 7-2 (a) and (b) show the amounts adsorbed during single solute adsorption with no humic acid, simultaneous adsorption with humic acid, and pre-loading with humic acid versus the initial MB concentrations for NIP and PAC 200, respectively. The amounts adsorbed are averages of three experimental replicates and the error bars represent the standard deviations. Data was adjusted to account for MB removed by humic acid in control samples containing no adsorbents, and represents only the MB removed by the adsorbent.

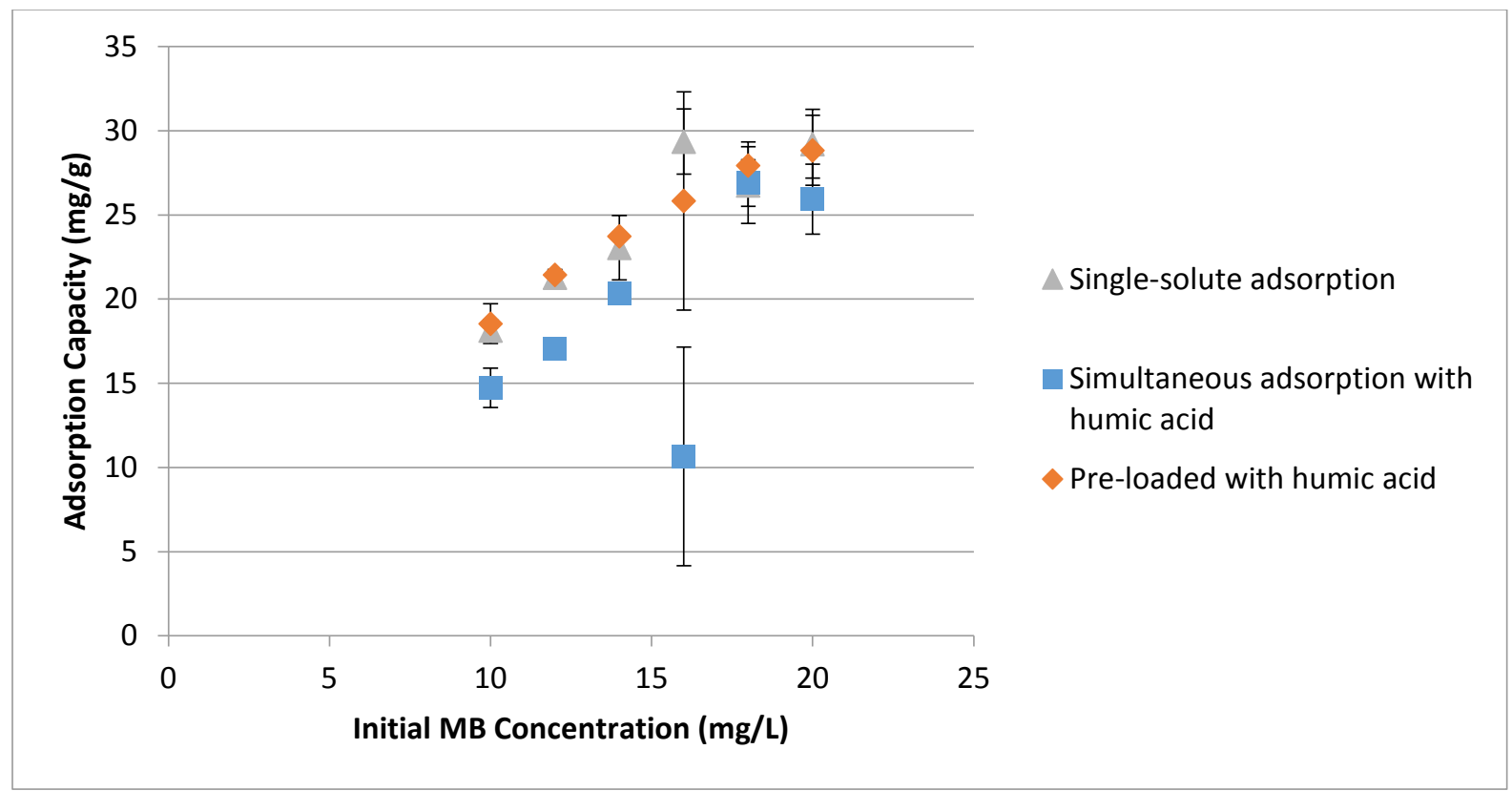

Figure 7-2 (a): Comparison between single-solute adsorption of MB and simultaneous adsorption and pre-loading with humic acid (20mg/L) for NIP 


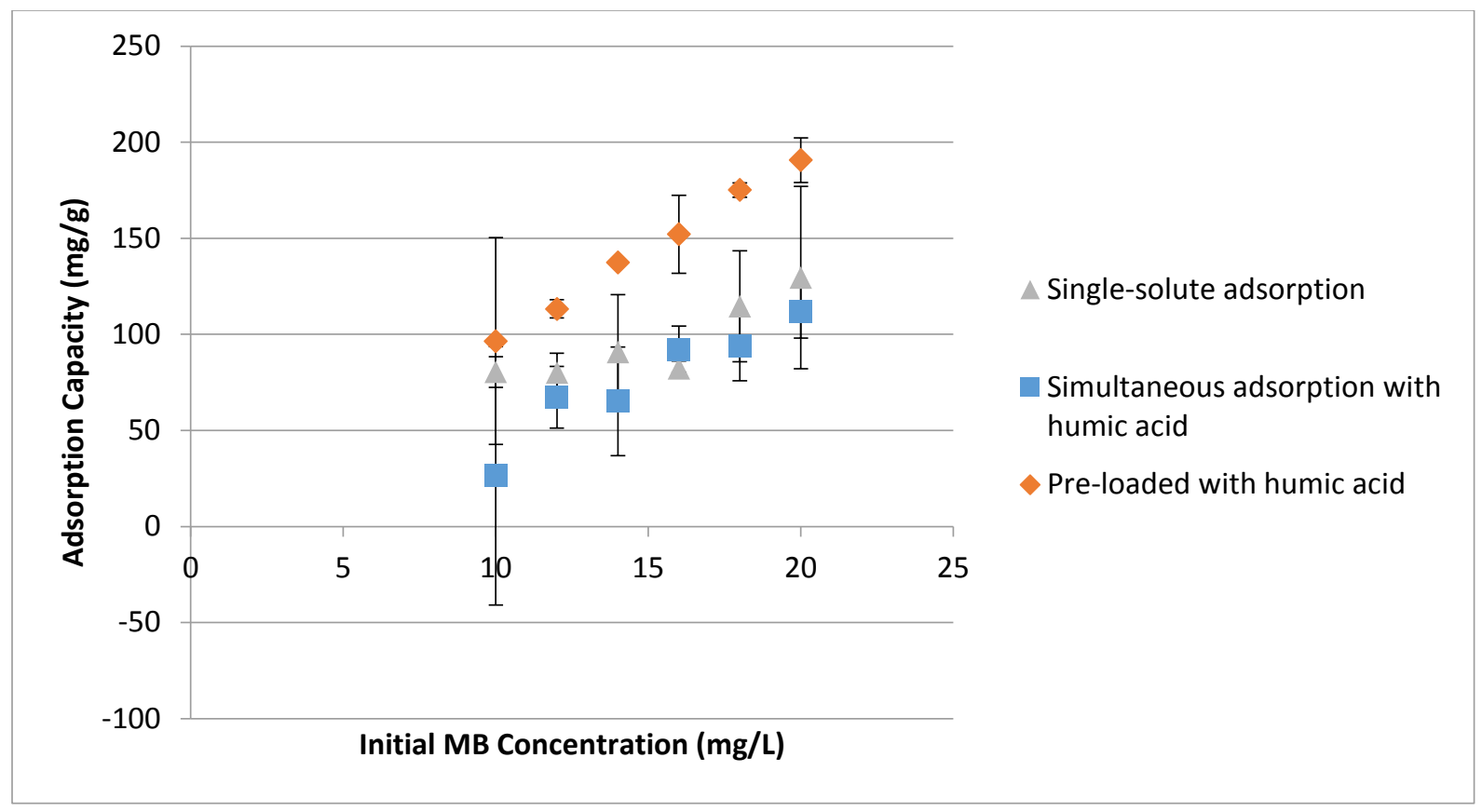

Figure 7-2 (b): Comparison between single-solute adsorption of MB and simultaneous adsorption and pre-loading with humic acid $(20 \mathrm{mg} / \mathrm{L})$ for PAC 200

To compare adsorption for single solute MB solutions to that in competition with humic acid, a t-test with a $90 \%$ confidence limit was used to conduct a paired comparison of means for changing initial MB concentrations. For the NIP during simultaneous adsorption with humic acid, adsorption was $27 \%(\mathrm{p}=0.087)$ lower with humic acid, which was significant for a $90 \%$ confidence limit. Similarly, for PAC 200, adsorption was $29 \%$ lower with the humic acid $(\mathrm{p}=0.096)$. Note that the adsorption capacity calculated for adsorption with humic acid for an initial MB concentration of $16 \mathrm{mg} / \mathrm{L}$ was an outlier, which may have increased the percent difference, but the results remain significant when this point is removed. These results indicate that there was competition between humic acid and MB for both NIP and PAC 200. The magnitude of the competitive effect was similar for both; 
adsorption of MB onto NIP decreased by $27 \%$ while adsorption onto PAC 200 decreased by $29 \%$.

In general, there are two mechanisms for competition between NOM and micropollutants: direct site competition and pore blocking. Direct site competition typically occurs when the adsorbate and NOM are similar in size and capable of occupying the same adsorption sites. In this case, the humic acid had an average molecular weight of $1716 \pm 478 \mathrm{~g} / \mathrm{mole}$ (by number) or $3303 \pm 904 \mathrm{~g} /$ mole (by mass) whereas MB has a molecular weight of 284 $\mathrm{g} /$ mole. It is unlikely that the humic acid would be capable of occupying the small pores energetically preferred by the MB molecules. Ebie et al (1995), found that volumes of pores ranging from 30-100 $\AA$ correlated best with adsorption of humic acid (Ebie, 1995). As stated previously, Pelekani et al (2000) found that adsorption of MB correlated well with micropore sizes close to $7.5 \AA$. Therefore, direct competition for adsorption sites is unlikely because size exclusion would prevent humic acid from occupying the same pores as the MB.

The NIP have a relatively low micropore volume, and had an average pore size of $73 \AA$. NIP also have a very low particle size of $217 \pm 4 \mathrm{~nm}$, and a relatively larger portion of their surface area is contained on the outside of the particles as compared to the activated carbon. Matsui et al (2012), compared a superfine activated carbon with conventionally sized activated carbons for adsorption of 2-methylisoborneol and NOM. Similar to NIP, the superfine activated carbon also contained a larger portion of its surface area on the outside surface of the activated carbon than the PAC did. They found that the superfine activated 
carbon was able to adsorb more NOM than the conventional activated carbon with a comparable decrease in 2- methylisoborneol capacity (Matsui et al., 2012). Likewise, many researchers have found that increasing the mesopore volume to allow for greater adsorption of humic acid actually increased micropollutant adsorption because humic acid adsorbed into the mesopores and secondary micropores, preventing pore blocking and leaving smaller micropores available for micropollutant adsorption (Pelekani and Snoeyink, 1999; Pelekani and Snoeyink, 2000; Redding and Cannon, 2014). Therefore, the presence of ideal pore sizes for humic acid adsorption does not necessarily indicate that the MB will have more competition from humic acid. However, the absence of smaller micropores in the NIP may mean that the MB is occupying adsorption sites in larger micropores and mesopores typically occupied by humic acid, and thus competing directly with humic acid.

A decreased adsorption capacity observed during simultaneous adsorption can be indicative of either direct competition for adsorption sites or pore blocking (Pelekani and Snoeyink, 1999). Although the larger molecules that block pores diffuse more slowly through solution, they block pores near the surface of the adsorbent and do not have to diffuse through the interior pore structure of the adsorbent to reach their final adsorption sites (Newcombe et al., 2002). However, a further decrease in adsorption performance following pre-loading with NOM, or exposure to NOM prior to exposure to the target adsorbate, is indicative of pore-blocking (Pelekani and Snoeyink, 1999).

Therefore, pre-loading experiments were conducted to further investigate the competitive mechanism. PAC 200 and NIP were exposed to $20 \mathrm{mg} / \mathrm{L}$ humic acid solutions for 24 hours, 
centrifuged, and then exposed to a MB solution for two weeks. These results are also shown in Figures 7-2 (a) and (b). Similar to the comparison presented between single-solute adsorption and simultaneous competition, t-tests with a $90 \%$ confidence limit were used to compare the average amounts adsorbed for each initial concentration.. As previously indicated, a decrease in adsorption between simultaneous and pre-loaded samples is indicative of pore blocking (Pelekani and Snoeyink, 1999).

For adsorption with NIP, shown in Figure 7-2 (a), the pre-loaded sample out-performed those that were simultaneously exposed. The NIP samples which were pre-loaded with humic acid performed $26 \%(\mathrm{p}=0.057)$ better than those that were simultaneously exposed to humic acid and $\mathrm{MB}$, which was significant for a $90 \%$ confidence limit. For adsorption with PAC 200, shown in Figure 7-2 (b), the pre-loaded activated carbon subsequently adsorbed 66\% $(\mathrm{p}=0.0008)$ more $\mathrm{MB}$ than was adsorbed during simultaneous exposure, which is significant for a $90 \%$ confidence limit. For both the NIP and PAC 200, adsorption increased for the pre-loaded sample in comparison to those that were simultaneously exposed to humic acid and MB. The difference could be caused by interactions between the activated carbon surface, humic acid, and MB. Referring to Table 7-1, the humic acid contained both carboxyl and phenolic functional groups. The MB dye may have interacted with these functional groups and adsorbed onto the humic acid. A comparison between single-solute adsorption and adsorbent pre-loading is required to determine which of these potential explanations is the most fitting. 
Comparisons were also made between pre-loading scenarios and single-solute adsorption, as shown in Figure 7-2. From Figure 7-2 (a) for NIP, it is clear that pre-loading with humic acid had no significant effect on subsequent adsorption of MB for a $90 \%$ confidence limit.

Figure 7-2 (b) indicates that the pre-loaded PAC 200 subsequently adsorbed more MB than was adsorbed during single-solute experiments. For a $90 \%$ confidence limit, the difference was $39 \%(\mathrm{p}=0.0005)$. This is clearly indicative of interactions between adsorbed humic acid and MB. Humic acids behave as polyelectrolytes in solution and have been shown to change the surface charge of activated carbons, making them more negative (Newcombe, 1994). This could reasonably increase adsorption of a cationic dye such as MB. The MB dye may also have adsorbed onto humic acid already adsorbed on the activated carbon by interacting with the carboxyl and phenolic functional groups on the humic acid. It does not mean that there was no competition for adsorption sites or pore blocking, but that the system was too complex to determine the degree of competition.

\subsubsection{Competitive adsorption with wastewater}

As discussed previously, natural organic matter, present in water and wastewater, can reduce the adsorption capacity of adsorbents by competing for adsorption sites or blocking pores. Competition with humic acid was studied because the sample was wellcharacterized, and, although the variability was large, the average molecular weight of the humic acid was known. However, humic acid represents only one fraction, typically the larger molecular weight fraction, of the natural organic matter present in wastewater, and wastewater also contains other organic and inorganic components which also compete with 
methylene blue during adsorption (Newcombe, 1994). Most significantly, it contains smaller molecules capable of accessing smaller pores occupied by MB. Adsorption of MB from wastewater using adsorbents pre-loaded with wastewater was examined to further evaluate competitive effects for NIP and PAC 200.

Figures 7-3 (a) and (b) show the amounts adsorbed during single solute adsorption with no humic acid, simultaneous adsorption with humic acid, and for samples pre-loaded with humic acid versus the initial MB concentrations for NIP and PAC-200, respectively. The amounts adsorbed are the averages of three experimental replicates and the error bars represent the standard deviations.

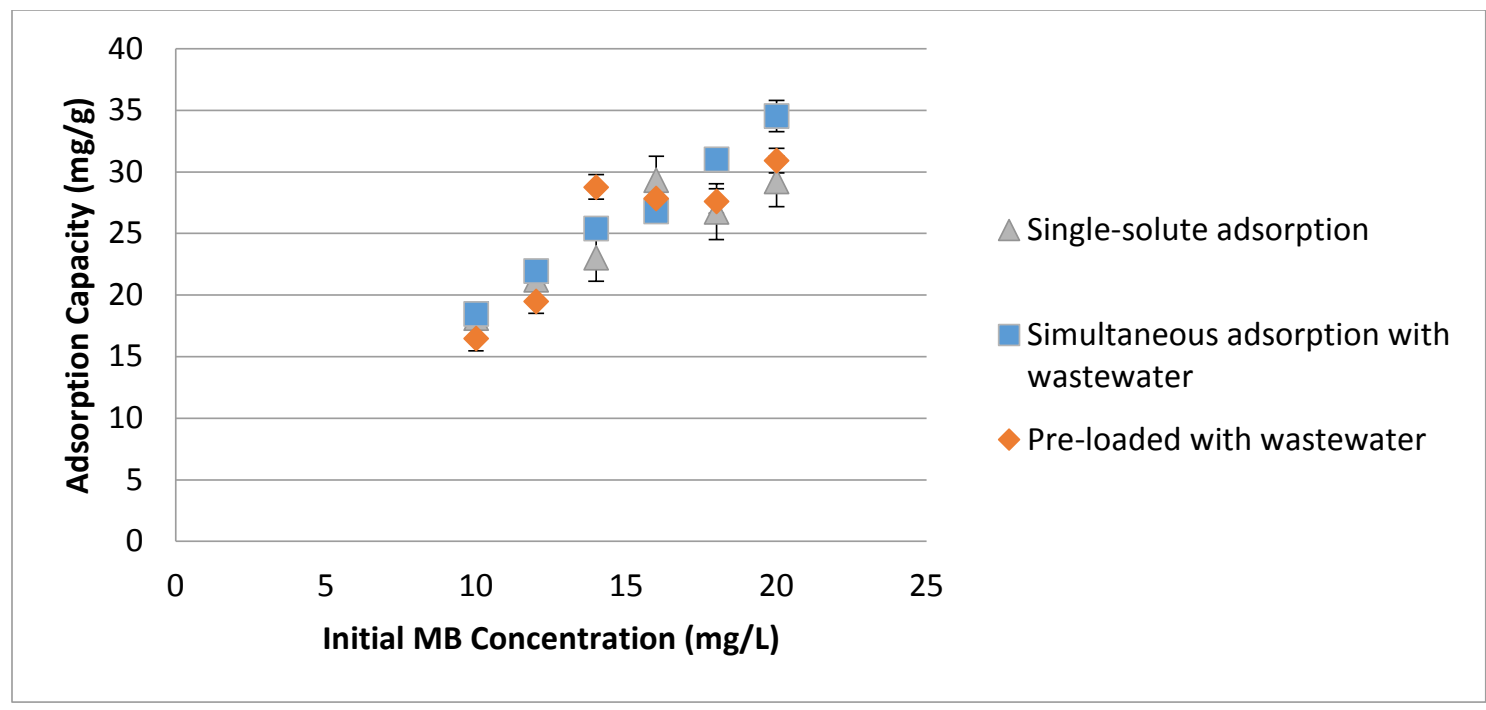

Figure 7-3 (a): Comparison between single-solute adsorption of MB and simultaneous adsorption and pre-loading with wastewater for NIP 


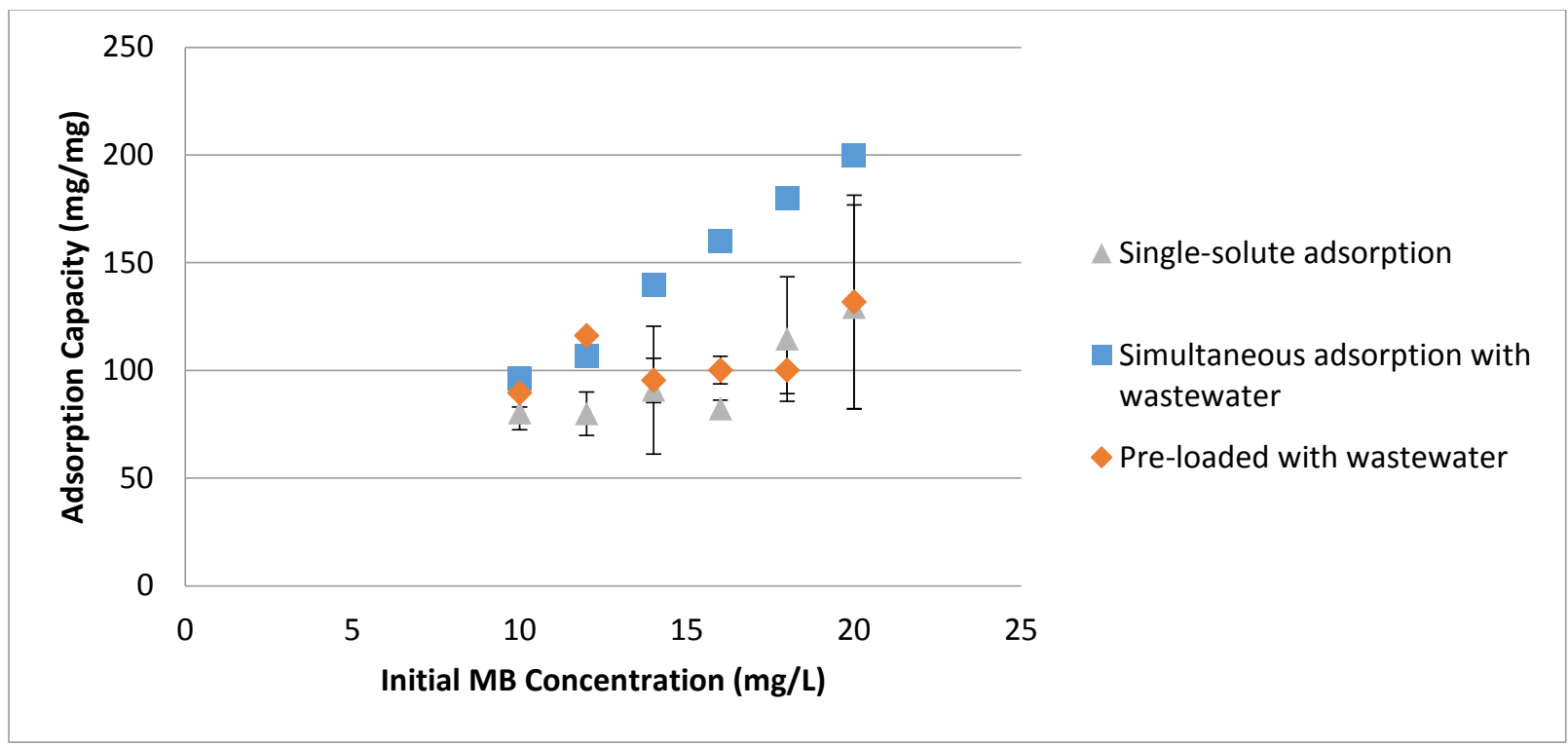

Figure 7-3 (b): Comparison between single-solute adsorption of $M B$ and simultaneous adsorption and pre-loading with wastewater for PAC 200

A t-test with a paired comparison of means was used to compare the amount of $\mathrm{MB}$ adsorbed from deionized water and secondary wastewater effluent with a $90 \%$ confidence limit. For the NIP, there was no significant difference for a $90 \%$ confidence limit for performance in wastewater versus deionized water $(\mathrm{p}=0.14)$. In fact, $20 \mathrm{mg} / \mathrm{L}$ of humic acid, which decreased the adsorption capacity by $27 \%(\mathrm{p}=0.09)$, had a more detrimental effect on MB adsorption onto NIP than the wastewater did. Humic acid has been shown to adsorb best in pores ranging from 30-100 $\AA$ (Ebie, 1995), and the average pore size of the NIP was $73 \AA$, which is within that range. Competitive adsorption of humic acid onto NIP may have been greater than that of wastewater for that reason. The wastewater contained a greater distribution in molecular size, and thus would have competed for a greater distribution of pore sizes. 
For PAC 200, adsorption of MB from wastewater was $40 \%(\mathrm{p}=0.001)$ higher than from deionized water and was significant for a $90 \%$ confidence limit. An increase in adsorption in the presence of competition was also seen for humic acid. Adsorption of NOM from wastewater can increase the negative surface charge of the adsorbent (Newcombe, 1994) and increase adsorption of $\mathrm{MB}$, which is a cationic dye.

Pre-loading with wastewater was compared to simultaneous exposure to wastewater and MB to investigate competition by pore-blocking. The differences between the samples were evaluated using a t-test with a $90 \%$ confidence limit. There was no significant difference between simultaneous exposure and pre-loading for NIP, shown in Figure 73(a) $(\mathrm{p}=0.3)$. For PAC 200 , the pre-loaded sample reduced adsorption by $30 \%(\mathrm{p}=0.0008)$ as compared to simultaneous exposure. However, the pre-loaded sample needs to be compared to single-solute adsorption from deionized water to determine whether this is an effect of competition, or simply a reduction in the enhanced adsorption caused by the change in surface charge due to adsorbed NOM from the wastewater.

Pre-loading with wastewater followed by adsorption of MB was compared to single-solute adsorption of MB dye from deionized water. There was no significant difference for a $90 \%$ confidence interval between the adsorbents pre-loaded with wastewater and fresh adsorbents for either NIP ( $\mathrm{p}=0.8)$ or PAC $200(\mathrm{p}=0.2)$. This could be because of an absence of competition from humic acid or enhanced adsorption caused by changes in the surface charge of the adsorbents shadowing negative effects of competition. 
It is clear from this work that experiments into competitive mechanisms between micropollutants and NOM cannot ignore the effects of surface chemistry and surface charge. MB dye is a basic, hydrophobic dye with a Kow value of 5.85, and as such, could potentially be used as a model for basic, hydrophobic micropollutants (NCBI, 6099). In this case, those effects became clear because the change in surface charge likely enhanced adsorption. However, it is possible for those changes to decrease adsorption instead (Newcombe, 1994), in which case they would be indistinguishable from competitive effects.

\subsubsection{Effect of zeta potential on adsorption performance for NIP and PAC 200}

Investigations into simultaneous adsorption of humic acid and MB onto NIP and activated carbon as well as adsorption of MB onto adsorbents pre-loaded with humic acid revealed competitive effects for simultaneous adsorption, but no decrease in MB adsorption for preloaded adsorbents. Both NIP and PAC 200 showed significant decreases during simultaneous exposure to humic acid and $\mathrm{MB}$, but there was no significant decrease for NIP pre-loaded with humic acid, and pre-loaded PAC 200 showed a significant increase in performance. These increases may have been due to an increase in the negative surface

charge of the adsorbents due to adsorbed humic acid, leading to increased adsorption of MB dye, which was a cationic dye. To further investigate the effect of surface charge on adsorption, the zeta potentials were measured for: (1) NIP and PAC 200 in deionized water; (2) NIP and PAC 200 in $20 \mathrm{mg} / \mathrm{L}$ of humic acid; and (3) NIP and PAC 200 pre-loaded with $20 \mathrm{mg} / \mathrm{L}$ of humic acid. The humic acid and adsorbent concentrations were the same as those studied during the single-solute and competitive adsorption experiments. Figure 7-4 
below, shows the zeta potential measurements for each case. The data shown are averages of 20 measurement replicates and the error bars indicate the standard deviation.

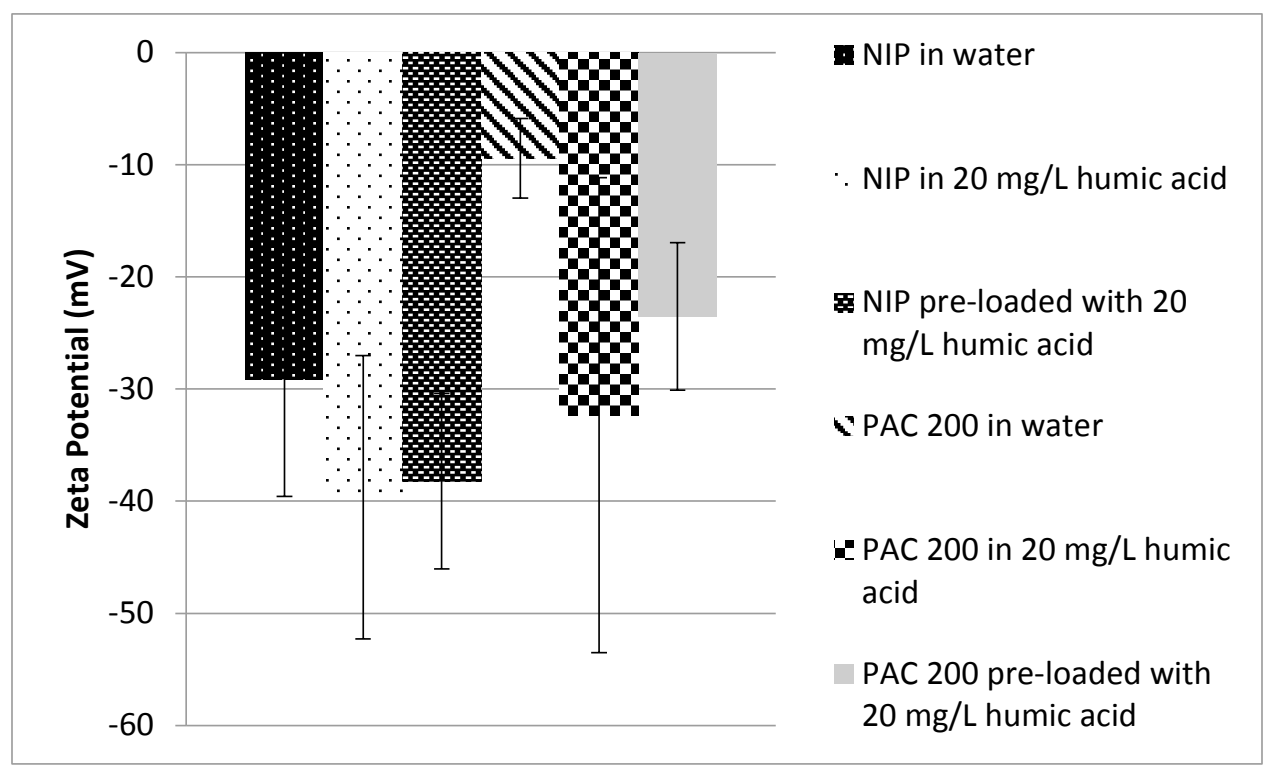

Figure 7-4: Effect of humic acid on the zeta potential of NIP and PAC 200

The zeta potential was measured using a Zeta-Meter 4.0. The Zeta-Meter uses backlighting to increase the visibility of colloidal particles that would normally require very high magnification. A microscope with magnification of $80 \mathrm{x}$ is sufficient to see the NIP particles which are $217 \pm 4 \mathrm{~nm}$ in diameter. The particles appear as bright dots under the microscope. A voltage is applied and the time that it takes a particle to travel a set distance is recorded. The error bars for some of the zeta potential measurements were large because the NIP were very small and difficult to see with the zeta potential microscope, and the activated carbon did not reflect light uniformly and, although the particles were easily visible, they 
tended to blink. There was also a noticeable difference in the speed at which individual particles travelled when the voltage was applied.

Although the change was within the range of the error bars, Figure 7-4 shows that the NIP particles did, in fact, become more negative in humic acid or when pre-loaded with humic acid than in water. The zeta potential decreased from $-29.16 \pm 10.42 \mathrm{mV}$ in water to -38.23 $\pm 7.82 \mathrm{mV}$ in $20 \mathrm{mg} / \mathrm{L}$ of humic acid and $-39.65 \pm 12.64 \mathrm{mV}$ pre-loaded with humic acid. Due to the size of the error bars, it is not possible to say conclusively that the particles became more negative, but the zeta potential measurements do support the results obtained during single-solute and competitive adsorption of MB where increased adsorption was observed for solutions with humic acid. Humic acid was expected to significantly decrease the performance of the NIP both during simultaneous adsorption with MB and when preloaded. However, although there was a significant decrease for simultaneous adsorption, there was no corresponding decrease for pre-loaded NIP. The zeta potential suggests that this may have been because the humic acid increased the negative surface charge of the NIP, and increased adsorption of MB. The decrease observed for simultaneous adsorption of $\mathrm{MB}$ and humic acid suggests that the humic acid competed with MB for adsorption sites or by blocking pores, decreasing adsorption, while also potentially increase the negative surface charge, increasing adsorption. For pre-loaded activated carbon the increase in adsorption caused by the increase in the negative surface charge may have cancelled out any competitive effects, leading to no significant difference between competitive adsorption and single-solute adsorption. 
The same phenomenon was also seen for PAC 200 in $20 \mathrm{mg} / \mathrm{L}$ of humic acid and preloaded with humic acid. Figure 7-4 shows that the zeta potential for PAC 200 decreased from $-9.41 \pm 3.55 \mathrm{mV}$ in water to $-32.33 \pm 21.16 \mathrm{mV}$ in $20 \mathrm{mg} / \mathrm{L}$ of humic acid, and -23.53 $\pm 6.57 \mathrm{mV}$ pre-loaded with humic acid. The error bars for the sample in humic acid were large, and the decrease was within the range of error, but those for the pre-loaded activated carbon were not, and the decrease was beyond the range of the error bars. The zeta potential measurements are supported by the adsorption experiments. Similar to NIP, PAC 200 showed a significant increase in performance for simultaneous exposure to MB and humic acid. Changes to zeta potential suggest that competitive effects may have been partially counteracted by an increase in the negative surface charge of the activated carbon. For activated carbon pre-loaded with humic acid, the increase in negative surface charge was enough to increase adsorption of MB dye.

\subsection{CONCLUSION}

This study compared NIP with three activated carbons (Norit PAC 200, Darco KB-M, and Darco S-51) for adsorption of methylene blue (MB) dye from water. Characterization of the NIP and the three activated carbons revealed that the NIP had a much smaller surface area of $2.4 \mathrm{~m}^{2} / \mathrm{g}$ compared to the powder activated carbons which ranged in surface area from 600 to $993 \mathrm{~m}^{2} / \mathrm{g}$. The NIP also had a smaller ratio of micropores to mesopores. The micropore to mesopore volume was 0.12 for the NIP versus 0.52 to 2.87 for the three activated carbons. The lower surface area and micropore to mesopore ratio of the NIP led to decreased adsorption capacity in comparison to the activated carbons. More porous NIP should be created to enhance their adsorption capacity. The NIP were also compared to 
Norit PAC 200 for adsorption of MB dye with competition from humic acid and wastewater. Competition occurred either during simultaneous exposure to the humic acid or wastewater and MB dye or pre-loading with humic acid or wastewater followed by subsequent adsorption of MB dye. Both the NIP and PAC 200 showed significant decreases of $27 \%$ for NIP $(\mathrm{p}=0.087)$ and $29 \%$ for PAC $200(\mathrm{p}=0.096)$ during simultaneous exposure to humic acid and methylene blue dye. Interestingly, there was no corresponding decrease, considering a 90\% confidence limit, for NIP or PAC 200 pre-loaded with humic acid and then exposed to MB. In fact, for PAC 200, the adsorption capacity of the activated carbon increased when it was pre-loaded with humic acid by $39 \%(\mathrm{p}=0.0005)$. This may have been due to an increase in the negative surface charge of the activated carbon caused by adsorbed humic acid. For wastewater, the NIP showed no significant increase or decrease in adsorption capacity during either simultaneous exposure or pre-loading for a $90 \%$ confidence limit. The adsorption capacity of PAC 200 increased by $40 \%(p=0.001)$ for simultaneous exposure to wastewater and MB. Pre-loading with wastewater had no effect on MB adsorption by PAC 200. Overall, the NIP and PAC 200 performed well in a variety of competitive situations with both humic acid and wastewater. It is not possible to draw conclusions about whether NIP or PAC were most affected by competition from NOM, and further investigation is required.

\subsection{REFERENCES}

Bergman, A., J. Heindel, S. Jobling, K. A. Kidd, and R. T. Zoeller. 2013 State of the science of endocrine disrupting chemicals - 2012. 
Bögi, C., J. Schwaiger, H. Ferling, U. Mallow, C. Steineck, F. Sinowatz, W. Kalbfus, RD Negele, I. Lutz, W. Kloas. 2003 Endocrine Effects of Environmental Pollution on Xenopus Laevis and Rana Temporaria. Environmental Research, 93 (2), 195-201. Cormack, P. a G., and A. Z. Elorza. 2004 Molecularly Imprinted Polymers: Synthesis and Characterisation. Journal of Chromatography. B, Analytical Technologies in the Biomedical and Life Sciences, 804 (1), 173-182.

Deng, S., D. Shuai, Q. Yu, J. Huang, and G. Yu. 2009 Selective Sorption of Perfluorooctane Sulfonate on Molecularly Imprinted Polymer Adsorbents. Frontiers of Environmental Science \& Engineering in China, 3 (2), 171-177.

Ding, L., V. L. Snoeyink, B. J. Mariñas, Z. Yue, and J. Economy. 2008 Effects of Powdered Activated Carbon Pore Size Distribution on the Competitive Adsorption of Aqueous Atrazine and Natural Organic Matter. Environmental Science \& Technology, 42 (4), 1227-31.

Ebie, K. 1995 Effect of Pore Size Distribution of Activated Carbon on the Adsorption of Humic Substances and Trace Organic Compounds. Water Supply, 13 (3-4), 65-70.

Ebie, K., F. Li, Y. Azuma, A. Yuasa, and T. Hagishita. 2001 Pore Distribution Effect of Activated Carbon in Adsorbing Organic Micropollutants from Natural Water. Water Research, 35 (1), 167-79.

Eduardo, B., and J. Tascon. 2008 Classical Methods for Pore Characterization. Adsorption by Carbons: Novel Carbon Adsorbents,. Elsevier, New York.

Ensing, K., and T. De Boer. 1999 Tailor-Made Materials for Tailor-Made Applications: Application of Molecular Imprints in Chemical Analysis. TrAC - Trends in Analytical Chemistry, 18 (3), 138-145. 
Fernández-Alvarez, P., M. Le Noir, and B. Guieysse. 2009 Removal and Destruction of Endocrine Disrupting Contaminants by Adsorption with Molecularly Imprinted Polymers Followed by Simultaneous Extraction and Phototreatment. Journal of Hazardous Materials, 163 (2-3), 1107-12.

Gillesby, B. E., and T. R. Zacharewski. 1998 Exoestrogens: Mechanisms of Action and Strategies for Identification and Assessment. Environmental Toxicology and Chemistry, 17 (1), 3-14.

Guo, W., W. Hu, J. Pan, H. Zhou, W. Guan, X. Wang, J. Dai, et al. 2011 Selective Adsorption and Separation of BPA from Aqueous Solution Using Novel Molecularly Imprinted Polymers Based on Kaolinite/Fe3O4 Composites. Chemical Engineering Journal, 171 (2), 603-611.

Hajizadeh, S., H. Kirsebom, I. Y. Galaev, and B. Mattiasson. 2010 Evaluation of Selective Composite Cryogel for Bromate Removal from Drinking Water. Journal of Separation Science, 33 (12), 1752-9.

Helfman, G. S. 2007 Fish Conservation: a Guide to Understanding and Restoring Global Aquatic Biodiversity and Fishery Resources, Island Press, Washington D.C.

International Humic Substances Society. 2015a Elemental Compositions and Stable Isotopic Ratios of IHSS Samples.

International Humic Substances Society. 2015b Acidic Functional Groups of IHSS Samples.

Krupadam, R. J., M. S. Khan, and S. R. Wate. 2010 Removal of Probable Human Carcinogenic Polycyclic Aromatic Hydrocarbons from Contaminated Water Using Molecularly Imprinted Polymer. Water Research, 44 (3), 681-8. 
Le Noir, M., A.-S. Lepeuple, B. Guieysse, and B. Mattiasson. 2007a Selective Removal of 17Beta-Estradiol at Trace Concentration Using a Molecularly Imprinted Polymer. Water Research, 41 (12), 2825-31.

Le Noir, M., F. Plieva, T. Hey, B. Guieysse, and B. Mattiasson. 2007b Macroporous Molecularly Imprinted Polymer/Cryogel Composite Systems for the Removal of Endocrine Disrupting Trace Contaminants. Journal of Chromatography. A, 1154 $(1-2), 158-64$.

Li, L., P. a Quinlivan, and D. R. U. Knappe. 2002 Effects of Activated Carbon Surface Chemistry and Pore Structure on the Adsorption of Organic Contaminants from Aqueous Solution. Carbon, 40 (12), 2085-2100.

Li, Q., V. L. Snoeyink, B. J. Mariãas, and C. Campos. 2003a Elucidating Competitive Adsorption Mechanisms of Atrazine and NOM Using Model Compounds. Water Research, 37 (4), 773-784.

Li, Q., V. L. Snoeyink, B. J. Mariñas, and C. Campos. 2003b Pore Blockage Effect of NOM on Atrazine Adsorption Kinetics of PAC: The Roles of PAC Pore Size Distribution and NOM Molecular Weight. Water Research, 37 (20), 4863-4872.

Li, Y., X. Li, Y. Li, J. Qi, J. Bian, and Y. Yuan. 2009 Selective Removal of 2,4Dichlorophenol from Contaminated Water Using Non-Covalent Imprinted Microspheres. Environmental Pollution, 157 (6), 1879-85.

Lin, Y., Y. Shi, M. Jiang, Y. Jin, Y. Peng, B. Lu, and K. Dai. 2008 Removal of Phenolic Estrogen Pollutants from Different Sources of Water Using Molecularly Imprinted Polymeric Microspheres. Environmental Pollution, 153 (2), 483-91. 
Luo, X., Y. Zhan, Y. Huang, L. Yang, X. Tu, and S. Luo. 2011 Removal of Water-Soluble Acid Dyes from Water Environment Using a Novel Magnetic Molecularly Imprinted Polymer. Journal of Hazardous Materials, 187 (1-3), 274-82.

Masqué, N. 2001 Molecularly Imprinted Polymers: New Tailor-Made Materials for Selective Solid-Phase Extraction. TrAC Trends in Analytical Chemistry, 20 (9), $477-486$.

Matsui, Y., Y. Fukuda, T. Inoue, and T. Matsushita. 2003 Effect of Natural Organic Matter on Powdered Activated Carbon Adsorption of Trace Contaminants: Characteristics and Mechanism of Competitive Adsorption. Water Research, 37 (18), 4413-4424.

Matsui, Y., T. Yoshida, S. Nakao, D. R. U. Knappe, and T. Matsushita. 2012 Characteristics of Competitive Adsorption Between 2-Methylisoborneol and Natural Organic Matter on Superfine and Conventionally Sized Powdered Activated Carbons. Water Research, 46 (15), 4741-4749.

Meng, Z., W. Chen, and A. Mulchandani. 2005 Removal of Estrogenic Pollutants from Contaminated Water Using Molecularly Imprinted Polymers. Environmental Science \& Technology, 39 (22), 8958-62.

Murray, Audrey. Evaluation of Molecularly Imprinted and Non-imprinted Nanoparticles for Removal of Endocrine Disrupting Compounds from Surface Water and Wastewater. Thesis. Carleton University, 2010. Ottawa: Carleton U, 2010. Print.

Newcombe, G. 1994 Activated Carbon and Soluble Humic Substances - Adsorption, Desorption, and Surface-Charge Effects. Journal of Colloid and Interface Science,. 
Newcombe, G., M. Drikas, and R. Hayer. 1997 Influence of Characterised Natural Organic Material on Activated Carbon Adsorption: II. Effect on Pore Volume Distribution and Adsorption of 2-Methylisoborneol. Water Research, 31 (5), 1065-1073.

Newcombe, G., J. Morrison, C. Hepplewhite, and D. R. U. Knappe. 2002 Simultaneous Adsorption of MIB and NOM onto Activated Carbon II: Competitive Effects. Carbon, 40 2147-2156.

National Center for Biotechnology Information. PubChem Compound Database; $\mathrm{CID}=6099, \quad$ https://pubchem.ncbi.nlm.nih.gov/compound/6099 (accessed September 10, 2015).

Pelekani, C., and V. . Snoeyink. 1999 Competitive Adsorption in Natural Water: Role of Activated Carbon Pore Size. Water Research, 33 (5), 1209-1219.

Pelekani, C., and V. L. Snoeyink. 2000 Competitive Adsorption Between Atrazine and Methylene Blue on Activated Carbon: The Importance of Pore Size Distribution. Carbon, 38 (10), 1423-1436.

Pelekani, C., and V. L. Snoeyink. 2001 Kinetic and Equilibrium Study of Competitive Adsorption Between Atrazine and Congo Red Dye on Activated Carbon: The Importance of Pore Size Distribution. Carbon, 39 (1), 25-37.

Perdue, E. M., and J. D. Ritchie. 2003 Surface and Groundwater Weathering, and Soils: Dissolved Organic Matter in Freshwaters. In H. Holland \& K. Turekian, eds., Treatise on Geochemistry,. Elselvier, Oxford.

Quantachrome Corporation. 2013 Nova e Series Brochure. Boynton Beach. 
Quantachrome Corporation. n.d. Application of QSDFT (Quenched Solid Density Functional Theory) - A Novel Density Functional Theory for an Accurate Pore Size Analysis of Disordered Porous Carbons. Powder Technote, 40.

Randhawa, M., I. Gartner, and C. Becker. 2007 Imprinted Polymers for Water Purification. Journal of Applied Polymer Science, 106 (5), 3321-3326.

Redding, A. M., and F. S. Cannon. 2014 The Role of Mesopores in MTBE Removal with Granular Activated Carbon. Water Research, 56 214-24.

Rochester, J. R. 2013 Bisphenol A and Human Health: A Review of the Literature. Reproductive Toxicology (Elmsford, N.Y.), 42 132-55.

Segner, H., K. Caroll, M. Fenske, C. R. Janssen, G. Maack, D. Pascoe, and C. Sch. 2003 Identification of Endocrine-Disrupting Effects in Aquatic Vertebrates and Invertebrates : Report from the European IDEA project 54 302-314.

Sellergren, B. 1999 Polymer-and Template-Related Factors Influencing the Efficiency in Molecularly Imprinted Solid-Phase Extractions. TrAC Trends in Analytical Chemistry, 18 (3), 164-174.

Wang, S., Z. H. Zhu, A. Coomes, F. Haghseresht, and G. Q. Lu. 2005 The Physical and Surface Chemical Characteristics of Activated Carbons and the Adsorption of Methylene Blue from Wastewater. Journal of Colloid and Interface Science, 284 (2), 440-446.

Wei, S., A. Molinelli, and B. Mizaikoff. 2006 Molecularly Imprinted Micro and Nanospheres for the Selective Recognition of 17Beta-Estradiol. Biosensors \& Bioelectronics, 21 (10), 1943-51. 
Xie, Y., H. Li, L. Wang, Q. Liu, Y. Shi, H. Zheng, M. Zhang, Y. Wu, B. Lu. 2011 Molecularly Imprinted Polymer Microspheres Enhanced Biodegradation of Bisphenol A by Acclimated Activated Sludge. Water Research, 45 (3), 1189-98.

Yan, H., and K. H. Row. 2006 Characteristic and Synthetic Approach of Molecularly Imprinted Polymer. International Journal of Molecular Sciences, 7 (5), 155-178.

Yang, M., M. S. Park, and H. S. Lee. 2006 Endocrine Disrupting Chemicals: Human Exposure and Health Risks. Journal of Environmental Science and Health. Part C, Environmental Carcinogenesis \& Ecotoxicology Reviews, 24 (2), 183-224.

Yu, Q., S. Deng, and G. Yu. 2008 Selective Removal of Perfluorooctane Sulfonate from Aqueous Solution Using Chitosan-Based Molecularly Imprinted Polymer Adsorbents. Water Research, 42 (12), 3089-97.

Zeta Meter Inc. 2010 Zeta Meter System 4.0 Operating Instructions. Staunton VA.

Zhongbo, Z., and J. Hu. 2008 Selective Removal of Estrogenic Compounds by Molecular Imprinted Polymer (MIP). Water Research, 42 (15), 4101-8. 


\section{CHAPTER 8: INVESTIGATION OF PHYSICAL SEPARATION PROCESSES FOR REMOVAL OF NON-IMPRINTED POLYMERS FOLLOWING WATER AND WASTEWATER TREATMENT}

\subsection{ABSTRACT}

Molecularly imprinted polymers (MIP) and non-imprinted polymers (NIP) are effective for removal of trace organic contaminants from both water and wastewater, but their small size (approximately $300 \mathrm{~nm}$ ) poses a challenge for full-scale application. Conventional separation methods for suspended solids such as filtration, centrifugation (for wastewater treatment), and ballasted flocculation were investigated for removal of NIP particles following treatment. Centrifugation at $10,000 \mathrm{rpm}(670800 \mathrm{xg})$ for 10 minutes, filtration with a $0.22 \mu \mathrm{m}$ filter, and ballasted flocculation achieved $99 \pm 1 \%, 92 \pm 3 \%$, and $88 \pm 3 \%$ removal, respectively. Due to the small size of the particles, accurately measuring the concentration of particles remaining in solution can also pose a challenge, and turbidity and particle size measurements were compared for this purpose. Turbidity was found to be a better measure of NIP concentration. NIP were also qualitatively tested for robustness over a $\mathrm{pH}$ range of 2 to 11 using SEM images, and no changes were visible between $\mathrm{pH} 2$ and 9. The results showed that conventional physical separation processes, commonly employed at treatment plants, are effective in removing NIP.

\subsection{INTRODUCTION}

Molecularly imprinted (MIP) and non- imprinted polymers (NIP) effectively treat emerging contaminants of concern such as endocrine disrupting compounds and pharmaceuticals in water and wastewater (Meng et al., 2005; Le Noir et al., 2007a; 
Randhawa et al., 2007; Le Noir et al., 2007b; Lin et al., 2008; Yu et al., 2008; Zhongbo and Hu, 2008; Deng et al., 2009; Fernández-Alvarez et al., 2009; Li et al., 2009; Hajizadeh et al., 2010; Krupadam et al., 2010; Li and Lai, 2010; Guo et al., 2011; Luo et al., 2011; Murray et al., 2011; Xie et al., 2011). Their small size (approximately $300 \mathrm{~nm}$ ) gives them a high surface area to volume ratio, which contributes to a high adsorption potential. However, removal of the small particles after treatment, presents a barrier to full-scale application, which must be overcome before their potential can be fully realized. Simple and cost-effective methods for application and removal of NIP are needed. Filtration, centrifugation (for wastewater treatment), and ballasted sand flocculation are versatile application options involving few structural changes.

Adsorption of trace organic contaminants from water and wastewater is typically achieved using activated carbon. One drawback to using activated carbon is that it functions by nonspecific adsorption and its performance is affected by the quality of the water to be treated and the presence of competing substances mainly organic matter (Fuerhacker et al., 2001). By contrast, MIP can specifically target a trace organic contaminant and have been shown to be equally effective in deionized water, river water, or wastewater (Lin et al., 2008). NIP are designed in the same way as MIP but are not specific to one contaminant and can simultaneously remove a wide range of trace organic contaminants.

Application of MIP in a column has been shown to be ineffective due to clogging, and other methods have been developed. Le Noir et al (2007) tested removal of 17- $\beta$ estradiol from wastewater effluent using a small column packed with $100 \mathrm{mg}$ of MIP and found that 
they were only able to filter $55 \mathrm{~mL}$ of wastewater at a very low flow-rate $(1 \mathrm{~mL} / \mathrm{min})$ before the column clogged (Le Noir et al., 2007b).

Currently two prevailing methods have been investigated for full-scale application of MIP and NIP for water and wastewater treatment. The first method involves the incorporation of the MIP (or NIP) into macroporous cryogels. The cryogels contain pores of varying sizes: smaller pores allow contact between the water and the MIP and larger pores allow for a high flow rate (Le Noir et al., 2007b). Cryogels allow for a higher wastewater flowrate with lower risk of clogging than MIP columns (Le Noir et al., 2007b). However, the incorporation of MIP and NIP into cryogels results in a structure very similar to that of activated carbon, where adsorption sites must be accessed through interconnected pores. Fukuhara et al (2006) suggested that much of the decrease in efficiency of activated carbon in wastewater came from pore blocking and not competition for adsorption sites, so the incorporation of MIP and NIP into a similar structure may not take full advantage of some of their benefits. The second method suggested for application of MIP and NIP is the creation of MIP or NIP with magnetic cores (Li et al., 2010; Luo et al., 2011; Xia et al., 2012a; Xia et al., 2012b). This method is particularly promising for industrial applications where it may be possible to recycle the adsorbate (Luo et al., 2011). Clogging or pore blocking would not be an issue for magnetic MIP; however, structural changes would be required to incorporate a strong magnet into the treatment scheme.

Filtration and centrifugation (for wastewater treatment) are already used in many treatment plants, although centrifugation is currently feasible only for small flowrates, and would 
allow the MIP or NIP to be added in a batch process particularly for small industrial flows with high concentrations of trace organic contaminants. Ballasted flocculation incorporates microsand into the coagulation-flocculation-settling regime typically used for water treatment to increase the size and density of the particles to be removed (Desjardins et al., 2002). It should be noted, however, that recovery of the NIP for reuse would not be possible with ballasted flocculation. Since the challenge in removing MIP and NIP comes from their small particle size, incorporating in dense sand will increase the removal through settling. Ballasted flocculation may also increase the effectiveness of filtration and centrifugation and could be used to treat large volumes of water such as those commonly seen in municipal wastewater treatment. Application of MIP or NIP with a combination of filtration, centrifugation, and ballasted flocculation has the potential to provide a highly versatile means of removing trace contaminants, particularly from industrial wastewater, by taking full advantage of the high surface area and specificity of the MIP or NIP.

The ruggedness and safety of MIP and NIP is another area of concern for treatment applications. If the MIP and NIP are used to treat water and wastewater, and they are not completely removed following treatment, it is possible that some of the particles will enter the surface water systems. Svenson et al (2001) studied the chemical stability of MIP polymers following exposure to a broad range of $\mathrm{pHs}$, organic bases, and an autoclave treatment. They measured the re-binding of the MIP template following these treatments as a means of investigating the ruggedness of the MIP. They found no change over a wide range of pHs (Svenson and Nicholls, 2001). For water and wastewater applications, high temperature is not expected to be a problem, but the MIP and NIP may be exposed to a 
range of $\mathrm{pHs}$ during treatment. It is important to investigate the ruggedness of MIP and NIP under a wide range of $\mathrm{pH}$ conditions. Also, re-binding of the template molecule may be indicative of the ruggedness of the MIP for re-use of the particles, but is not necessarily indicative of safety, other methods of evaluation are required

The objective of this work was to determine whether conventional methods such as centrifugation (for wastewater treatment), filtration, and ballast sand flocculation can be used to effectively remove MIP and NIP particles following treatment. Due to their small size, measuring particle concentrations to determine removal efficiency also posed a challenge. Thus, turbidity, zetasizer, and particle counter measurements were all used along with scanning electron microscope (SEM) imaging to gain insight into the behaviour of the particles under different removal regimes. The ruggedness of the particles under a wide range of $\mathrm{pH}$ conditions was also investigated to evaluate their potential for full-scale application. It should be noted that MIP and NIP technologies are still in their development

phase, and the best method of application has not yet been determined. The following discussion is intended to contribute to that discussion and is not intended to provide final recommendations regarding the ideal method of application.

\subsection{MATERIALS AND METHODS}

\subsubsection{Preparation of NIP solution}

Functional monomer methacrylic acid (MAA) (Sigma-Aldrich; Oakville, Canada) and cross-linker ethylene glycol dimethacrylate (EGDMA) (Sigma-Aldrich; Oakville, Canada) were dissolved in a porogen with a molar ratio of $8 \mathrm{mmol}: 6.7 \mathrm{mmol}$ (Wei et al., 2006). The 
porogen was composed of $40 \mathrm{~mL}$ of 1:3 (v:v) acetone (Fisher Scientific; Ottawa, Canada), and acetonitrile (Fisher; Ottawa, Canada). $2 \%$ (w:w) of 2- isobutryonitrile (AIBN) was added as the initiator (Sigma-Aldrich; Oakville, Canada). The mixture was mixed with a vortex mixer (Fisher Scientific Vortex Mixer, USA), deoxygenated with nitrogen for five minutes, and then placed in a $60^{\circ} \mathrm{C}$ hot water bath for $24 \mathrm{~h}$ (Isotemp 220, Fisher, USA). The resulting polymer particles were dewatered using a centrifuge (Thermo Scientific Sorval Legend $\mathrm{RT}^{+}$, Fisher Scientific) at 10,000 RPM, air dried at room temperature, and ground manually.

NIP were used for all removal experiments because they are smaller than the MIP, meaning that they should theoretically be more difficult to remove and present a most-challenging scenario. They are also easier to prepare because they do not require the addition and subsequent removal of a template molecule. An NIP solution was prepared by sonicating the NIP (Vibracell Sonics, Sonics and Materials Inc., Newtown Connecticut) in $1 \mathrm{~L}$ of water for 15 minutes to disperse the NIP before being diluted to a final concentration of 5 $\mathrm{mg} / \mathrm{L}$. The solution was stirred thoroughly before being dispensed.

\subsubsection{Removal of NIP with gravity settling}

To determine the settleability of NIP with no treatment, three $1 \mathrm{~L}$ beakers of the $5 \mathrm{mg} / \mathrm{L}$ NIP suspension in tap water were monitored for 24 hours. Samples were taken $5 \mathrm{~cm}$ below the surface of the water and measured for turbidity. Tap water was used to evaluate the feasibility of various treatment methods because of the large volume of water required. Refer to Appendix B for a summary of the water characteristics. 


\subsubsection{Removal of NIP with filtration}

A lab-scale filtration set-up was used to evaluate the effectiveness of varying filter pore sizes on NIP removal. The pore sizes investigated were $0.22 \mu \mathrm{m}$ (using a nylon syringe filter from Derian); $0.7 \mu \mathrm{m}$ (GF/F glass microfiber filters, Whatmann); $1 \mu \mathrm{m}$ (GF/B glass microfiber filters, Whatmann); $1.6 \mu \mathrm{m}$ (G6 glass fiber circles, Fisher); and $3 \mu \mathrm{m}$ (Grade 6 qualitative filters, Whatmann).

\subsubsection{Removal of NIP with centrifugation}

Centrifuge time and speed were varied to determine optimum conditions for removal of NIP. The NIP solution was prepared as outlined above and poured into three $50 \mathrm{~mL}$ tubes. The centrifuge (Thermo Scientific Sorval Legend $\mathrm{RT}^{+}$, Fisher Scientific) was run for varying times and speeds. Then a $20 \mathrm{~mL}$ sample was pipetted from the surface. In the first set of experiments, the centrifuge time was varied for a constant speed of $10,000 \mathrm{rpm}$ corresponding to 670,800 times the force of gravity $(\mathrm{g})$. In the second set of experiments, the centrifuge speed was varied for a constant time.

\subsubsection{Removal of NIP with ballasted flocculation}

The jar test procedure for ballasted flocculation was followed as described by Desjardins et al (2001). A jar test apparatus from Phipps and Bird (Richmond, Virginia) was used with a flat blade impeller. The mixing speed was kept constant throughout and the coagulants, microsand, and polymer were added as follows: (1) at time $\mathrm{t}=0$, the coagulant was added and the jar tester started; (2) at time $\mathrm{t}=2$, the microsand and a percentage of the polymer 
were added; (3) at time $t=4$, the remainder of the polymer was added; (4) at time $t=10$ the mixing was turned off and the NIP were allowed to settle by gravity; and (5) at time $\mathrm{t}=13$, a sample was taken approximately $5 \mathrm{~cm}$ below the surface. The times were varied in some cases to achieve optimum performance but the order of the additions was maintained. The speed of impeller rotation for the jar-tester remained constant throughout. PH was allowed to vary with coagulant dose.

\subsubsection{Chemicals and sand for ballasted flocculation}

The coagulant used for ballasted flocculation was alum (CAS 7784-31-8) (Anachemia, Montreal, Quebec). The Polymer was Flopolymer CA4800 (SNF, Trois Rivieres, Quebec). Microsand samples were obtained from John Meunier Inc. (Montreal, Quebec). Alum was chosen as the coagulant because it is the most commonly used coagulant for water treatment (Crittenden, 2005). The polymer chosen was chosen because it is a high molecular weight cationic polymer and the NIP particles were negatively charged.

\subsubsection{Effect of settling time}

To illustrate the effect that ballasted flocculation had on NIP settleability, the NIP solution was allowed to settle for $1 \mathrm{~h}$ following treatment and the turbidity was monitored with time.

\subsubsection{Control parameters}

Since there were many factors (alum dose, polymer dose, the percent of the polymer added initially, sand dose, mixing intensity, coagulation time, flocculation time, and settling time) capable of influencing the effectiveness of the ballasted flocculation treatment, a statistical 
approach employing a $2^{8-4}$ factorial design was used to determine which factors had a significant impact on performance. A linear regression model was obtained from the results.

\subsubsection{Optimization of ballasted flocculation conditions}

Using the linear regression model obtained from the factorial design experiments, a path proportional to the coefficients in the regression model was followed until an increase in turbidity was observed. The conditions corresponding to the step before the increase in turbidity were taken as the optimum conditions.

\subsubsection{Effect of alum dose}

To compare the effectiveness of ballasted flocculation for tap water and wastewater, the effect of varying alum dose on ballasted flocculation was compared for both. The procedure for ballasted flocculation was followed as outlined above. For tap water, the alum was dispensed from a $1 \mathrm{~g} / \mathrm{L}$ stock solution and for wastewater a $10 \mathrm{~g} / \mathrm{L}$ stock solution was used due to the higher alum concentrations required for wastewater. The wastewater used was secondary effluent collected from the Robert O. Pickard Environmental Centre in Ottawa, Ontario.

\subsubsection{Scanning electron microscope (SEM) images}

A Tescan Vegall XMU SEM instrument was used to obtain SEM images. The NIP were coated with gold prior to imaging using RF sputter (Anatech Hummer, Union City, CA, USA). The images were collected at a working distance of 7-8mm. 


\subsubsection{Evaluation of removal efficiency with various analytical devices}

\subsubsection{Turbidity measurements}

The amount of NIP removed was evaluated using before and after comparisons of turbidity. Turbidity measurements were taken using a Hach 2100 AN turbidity meter.

\subsubsection{Particle size and distribution as measured by the DPA 4100 particle analysis system}

A DPA 4100 Particle Analysis System (Brightwell Technologies, Ottawa, ON) was used to obtain all particle count measurements. The DPA 4100 Particle Analysis system analyzes samples which are passed through a flow cell capillary device while light is shone on them. The resulting signal is optically magnified and projected onto a photo diode array detector (Thomas and Moore, 2004). It can quickly and accurately provide particle size distributions and particle concentration measurements, and simultaneously gathers images of the sample stream, allowing for characterization of particle types. $10 \mathrm{~mL}$ samples were stirred continuously as $2 \mathrm{~mL}$ sub-samples were drawn through the flow cell capillary and injected into the sampling syringe at a flowrate of $0.22 \mathrm{~mL} / \mathrm{min}$. Particle size distributions

were measured, and images of the sample water were gathered. Results reported are the average of two experimental replicates each of which consisted of three analytical replicates.

\subsubsection{Particle size as measured by the Nano ZS Zetasizer}

A Malvern Nano ZS Zetasizer was used to obtain particle size distributions and measure the average particle size before and after NIP removal. Two experimental replicates, each 
consisting of two analytical replicates, were conducted. The Nano ZS Zetasizer uses light scattering to measure the size of particles in a solution. A laser is directed towards the sample cell, and the light scattered at $173^{\circ}$ is measured. This angle means that the light does not have to travel through the entire sample, so multiple scattering, where light that has already been scattered by one particle hits another particle, as well as interference from dust and other contaminants, is avoided. Dust particles are usually large, and tend to scatter in a forward direction, and would not be detected at this angle (Malvern Instruments, 2000). Measurements taken using the Nano ZS Zetasizer complimented those provided by the DPA 4100 Particle Analysis System because the Nano ZS Zetasizer can measure particle sizes from $0.3 \mathrm{~nm}$ to $10 \mu \mathrm{m}$ in diameter, whereas the DPA 4100 does not accurately measure particles below $2 \mu \mathrm{m}$ in diameter. The Zetasizer was able to provide accurate size distributions before and after NIP removal, but could not provide particle concentrations, which were provided by the DPA 4100 .

\subsubsection{Exposure of NIP to acidic and basic conditions}

$20 \mathrm{mg}$ samples of NIP were added to $1.5 \mathrm{~mL}$ centrifuge tubes and $1 \mathrm{~mL}$ of water of varying $\mathrm{pH}(2-11)$ was added to each tube. The $\mathrm{pH}$ solutions were prepared using deionized water and concentrated sodium hydroxide or hydrochloric acid solutions (18\% and 36.5-38\%, respectively). The tubes were shaken to mix and left for 24 hours before the NIP were centrifuged and dried at room temperature. It should be noted that the $\mathrm{pH}$ range investigated was much larger than that encountered during water or wastewater treatment but was a test of extreme conditions. 


\subsection{RESULTS AND DISCUSSION}

\subsubsection{Removal of NIP with filtration}

The first method investigated for removal of MIP and NIP was filtration. Adsorption of trace contaminants onto MIP followed by removal of the MIP by filtration may appear to complicate treatment of trace contaminants such as pharmaceuticals and endocrine disruptors, which can be removed directly with nanofiltration or reverse osmosis. Yet, MIP and NIP are much larger than the contaminant molecules and could be removed with microfiltration. Braeken et al (2009) evaluated the use of nanofiltration for removal of endocrine disrupting compounds and found that it was not economically feasible (Braeken and Van der Bruggen, 2009). Since the cost of filtration decreases with increasing pore size, adsorption of the compounds of interest onto the NIP followed by microfiltration may be more cost effective than direct membrane removal with nanofiltration or reverse osmosis alone.

Figure 8-1 shows the removal of NIP using a lab-scale filtration set-up with filter pore sizes ranging from 0.22 to $3 \mu \mathrm{m}$. Filtration experiments were conducted with filter paper rather than granular media, which are commonly used in water treatment plants, and the results

would have to be correlated with those for granular media prior to application, but do show the potential for treatment. Turbidity measurements were used to determine the degree of removal. As expected, the removal of the NIP increased with decreasing pore size. The $3 \mu \mathrm{m}$ filters removed $32 \pm 12 \%$ of the NIP and the $0.22 \mu \mathrm{m}$ filters removed $92 \pm 3 \%$ of the NIP. Since the NIP used in this study have an average particle size of $333 \pm 76 \mathrm{~nm}$ (from SEM images), it was expected that the $0.22 \mu \mathrm{m}$ filters would remove most but not all of 
NIP. However, even the $0.7 \mu \mathrm{m}$ filters were very effective at removing the NIP and were capable of removing $84 \pm 3 \%$.

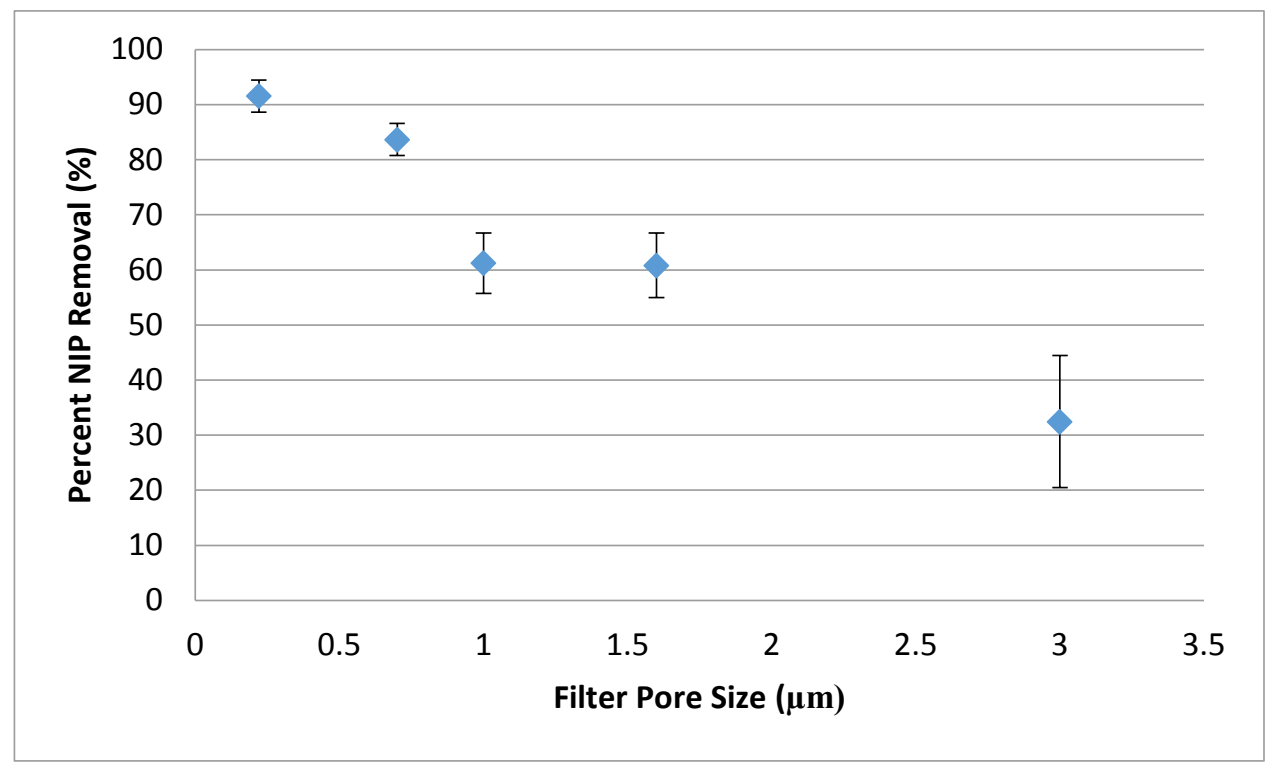

Figure 8-1: Percent removal of NIP with filtration based on turbidity measurements

There are several reasons why filter sizes larger than $0.3 \mu \mathrm{m}$ might retain a fraction of the NIP. Pore blocking from NIP that have already been retained will reduce the effective pore size or electrostatic charges may increase NIP retention. Also, although the NIP were fairly homogeneous in size and shape as seen from scanning electron microscope images (Murray et al., 2011), the NIP may not have been completely separated in solution, and groups of NIP could have been retained on much larger filter sizes. 


\subsubsection{Removal of NIP with centrifugation}

Centrifugation was also explored as a means of removing NIP following wastewater treatment. Centrifuge treatment would be limited to treatment of smaller flow volumes such as for industrial wastewater treatment, but were investigated because of its effectiveness for particle removal. Figure 8-2 a and b show the centrifuge removal of NIP. Figure 8-2 a shows the removal for a constant centrifuge time (3 minutes) and varying centrifuge speed $(5,000-10,000 \mathrm{rpm})$, and Figure 8-2 b shows the results for a constant centrifuge speed $(10,000 \mathrm{rpm}$ corresponding to $670,800 \mathrm{~g})$ and varying centrifuge time (110 minutes). The percent removal in each case was determined by measuring the turbidity before and after centrifugation for three replicates and recording the average.

Figure 8-2 a shows the results for a constant centrifuge time of 3 minutes and varying centrifuge speeds of 5,000-10,000 rpm. The percent removal increased distinctly for increasing centrifuge speeds between 5000 and $7000 \mathrm{rpm}$, from $49 \pm 10 \%$ to $80 \pm 7 \%$. The increase was then more gradual for $8000-10000 \mathrm{rpm}$ from $84 \pm 4 \%$ to $94 \pm 6 \%$. As presented in Figure 8-2 b, the percent removal increased sharply for increasing centrifuge times from 0-3 minutes, increasing from $36 \pm 75$ after 1 minute to $94 \pm 6 \%$ after 3 minutes. The increase in percent removal was more gradual for longer centrifuge times, reaching $99 \pm$ $1 \%$ for a 10 minute centrifuge time. From these results, it is clear that given a centrifuge capable of applying enough centrifugal force or for a long enough time, near complete removal of MIP and NIP following treatment is possible. 


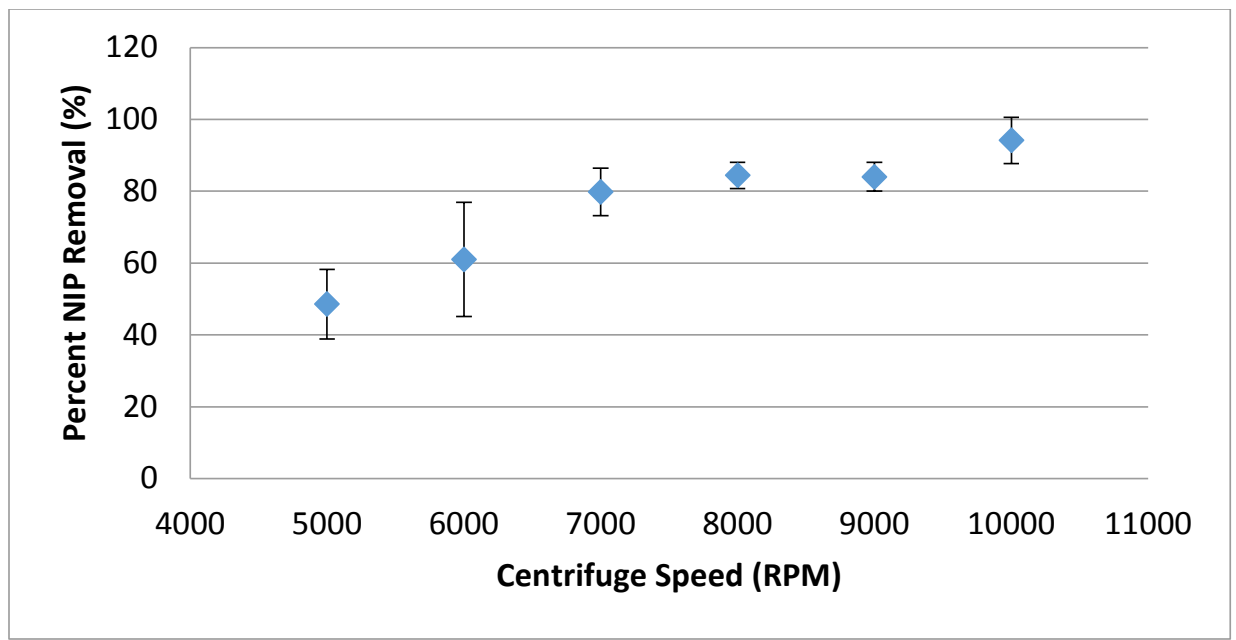

Figure 8-2 (a): Percent removal of NIP by centrifugation at varying centrifuge speeds for 3 minutes based on turbidity measurements

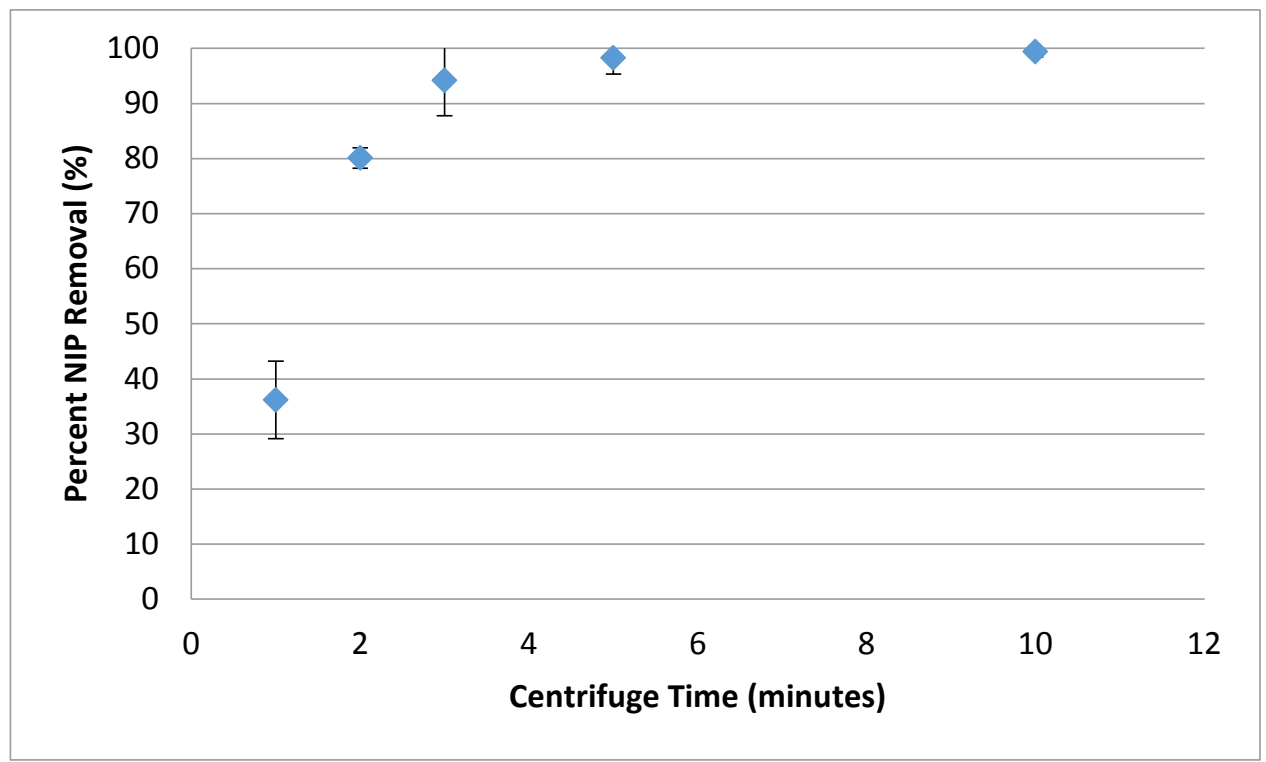

Figure 8-2 (b): Percent removal of NIP by centrifugation at 10,000 RPM for varying centrifuge times based on turbidity measurements 


\subsubsection{Removal of NIP with ballasted flocculation}

As demonstrated above, NIP particles can be removed using filtration and centrifugation based separation processes, which can be ideal for the treatment of side streams or industrial wastewater where the wastewater flow is smaller but has high concentrations of contaminants. However, they would not be feasible for treatment of large flows such as those seen for domestic wastewater. Therefore, the next step investigated the use of enhanced flocculation processes for the removal of NIP through gravity settling, which could be applied at conventional wastewater treatment plants to treat large volumes of wastewater.

Application and removal of MIP and NIP is difficult primarily due to their small size. Ballasted flocculation encourages the flocculation of MIP or NIP and increases the weight of the flocs by incorporating a microsand with a density of approximately $2700 \mathrm{~kg} / \mathrm{m}^{3}$ (Desjardins et al., 2002). Increasing the weight of the MIP and NIP through the formation of higher-density flocs then allows them to be removed easily with gravity settling. A jartest procedure simulating an ACTIFLO (Veolia Inc.) ballasted flocculation process applied in some full-scale drinking water treatment plants was tested as described by Desjardins et al (2002).

Turbidity was used to measure the reduction in NIP concentration during treatment, and the results shown represent an average of 3 replicates. Figure 8-3 shows the settling performance of the NIP with no added coagulants, polymers, or sand. Over a 24 hour period, the maximum settling was $22 \pm 8 \%$. Figure $8-3$ shows the results from ballasted 
flocculation over a range of settling times. The percent removal of the NIP after a 10 minute settling time was $77 \pm 1 \%$ and further extending the settling time did not significantly improve the performance. From these results, it is clear that ballasted flocculation can lead to better settling performance of NIP in a shorter time.

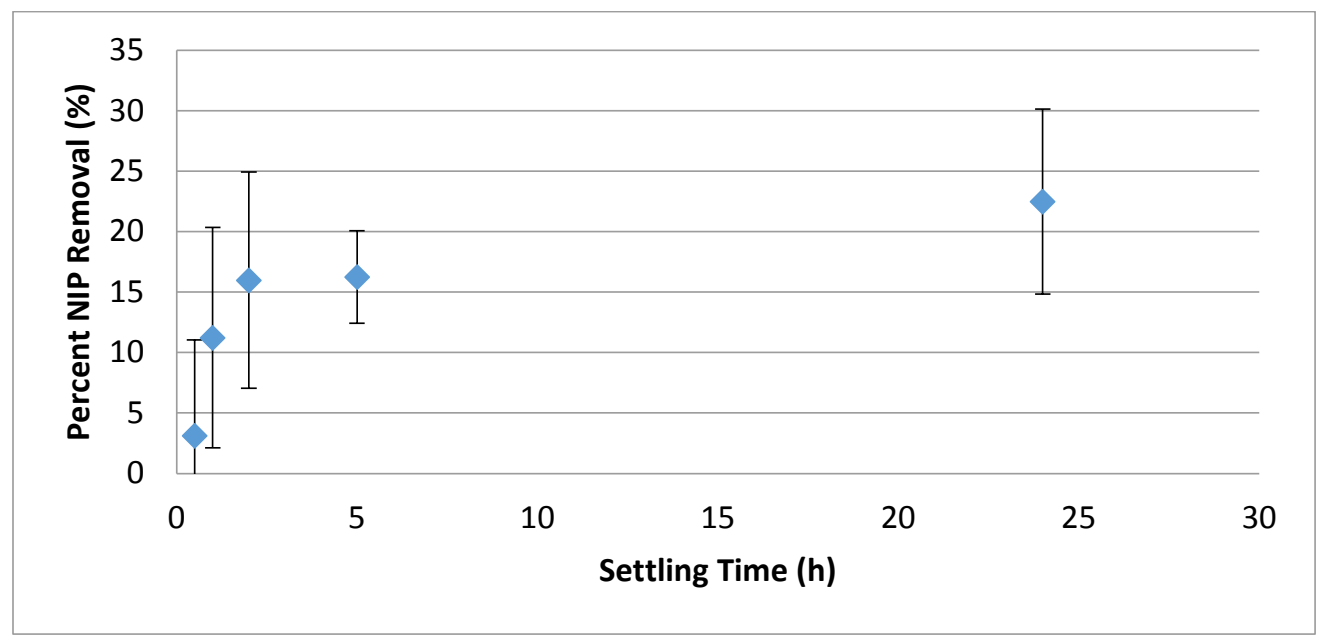

Figure 8-3 (a): Percent removal of NIP by gravity settling for extended settling times based on turbidity measurements

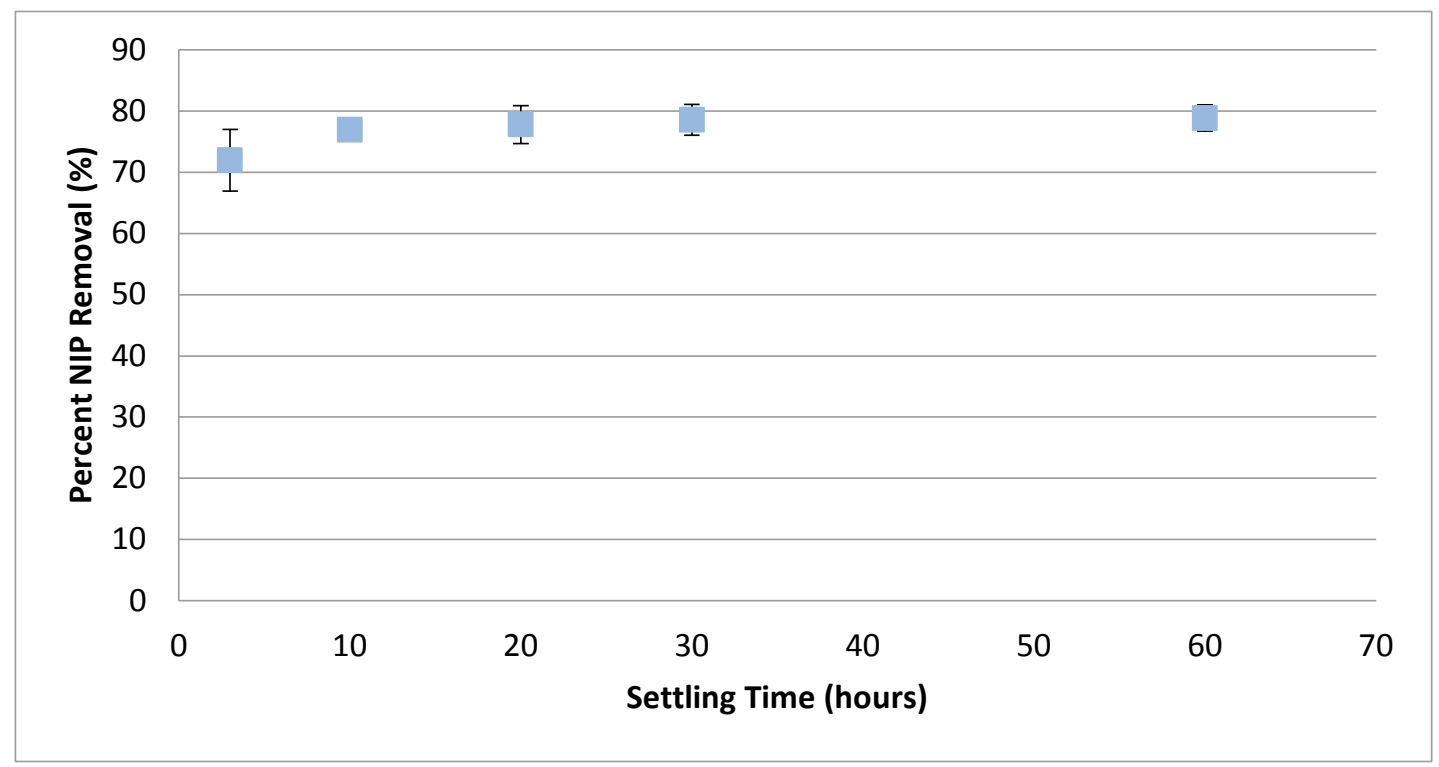


Figure 8-3 (b): Percent removal of NIP by ballasted flocculation with extended settling times based on turbidity measurements

Based on the excellent removal efficiency obtained using ballasted flocculation and gravity settling, and its potential for simple and cost-effective application at treatment plants, further experiments were carried out to determine the optimum conditions for NIP removal.

Since a ballasted flocculation process contains many design parameters which could be optimized, a $2^{8-4}$ factorial design was used to determine the most significant design variables. The ballasted flocculation procedure consists of 5 steps. First, the coagulant is added. Then, in step 2, the microsand and a percentage of the polymer are added. In step 3 , the remainder of the polymer is added. In step 4 , the mixing is turned off and the flocs are allowed to settle, and in step 5, a sample is taken. All parameters related to process operation were considered and included: alum dose, polymer dose, the fraction of the polymer added concurrently with the sand, sand dose, mixing intensity, coagulation time, flocculation time, and settling time. The alum dose, mixing intensity, and coagulation time were found to influence the final turbidity of the treated water as described by the linear regression model shown in Equation 1. Based on the values of the coefficients, alum dose and coagulation time were most important, and the mixing intensity, although it had a significant effect, was less important. Based on these results of the ANOVA test (shown in Appendix H), the polymer dose, fraction of polymer added with the sand, sand dose, flocculation time, and settling time did not influence the percent of NIP removed. However, the results are specific to the range of parameters tested, and they may have impacted the percent removal over a different range. 
Equation 1: Turbidity $=2.52-0.35$ alum dose -0.049 mixing intensity -

\subsection{6 coagulation time}

To optimize the alum dose, mixing intensity, and coagulation time, a path proportional to the regression coefficients in Equation 1 was followed until an increase in turbidity was observed. The optimized conditions led to a NIP removal of $88 \pm 3 \%$. It should be noted that optimum conditions are dependent on additional factors such as water characteristics or hydraulics, which are relevant for full-scale but not for jar tests and not considered in this study. The optimum conditions determined with the jar-test procedure may not necessarily correspond to the optimum in a full-scale system, but provide a general idea of the degree of removal possible. Nonetheless, Desjardins et al (2002) found good agreement between the performance of their jar-test procedure and three full-scale plants, with only a 7\% difference (Desjardins et al., 2002).

During the factorial experiment, alum dose had the most significant effect on performance. Figure 8-4 a and $\mathrm{b}$ show the effect of increasing alum dose for ballasted flocculation applied to $5 \mathrm{mg} / \mathrm{L}$ solutions of NIP in water and wastewater, respectively. Figure 8-4 a shows the effect of increasing alum dose for NIP in tap water. Increasing the alum dose from 4 to 6 $\mathrm{mg} / \mathrm{L}$ led to a substantial increase in performance from $9 \pm 4 \%$ removal with $4 \mathrm{mg} / \mathrm{L}$ to 72 $\pm 2 \%$ with $6 \mathrm{mg} / \mathrm{L}$ of alum. The turbidity continued to decrease at a slower rate as the alum dose was further increased and a maximum percent removal of $87 \pm 5 \%$ was reached with $8 \mathrm{mg} / \mathrm{L}$ of alum. 


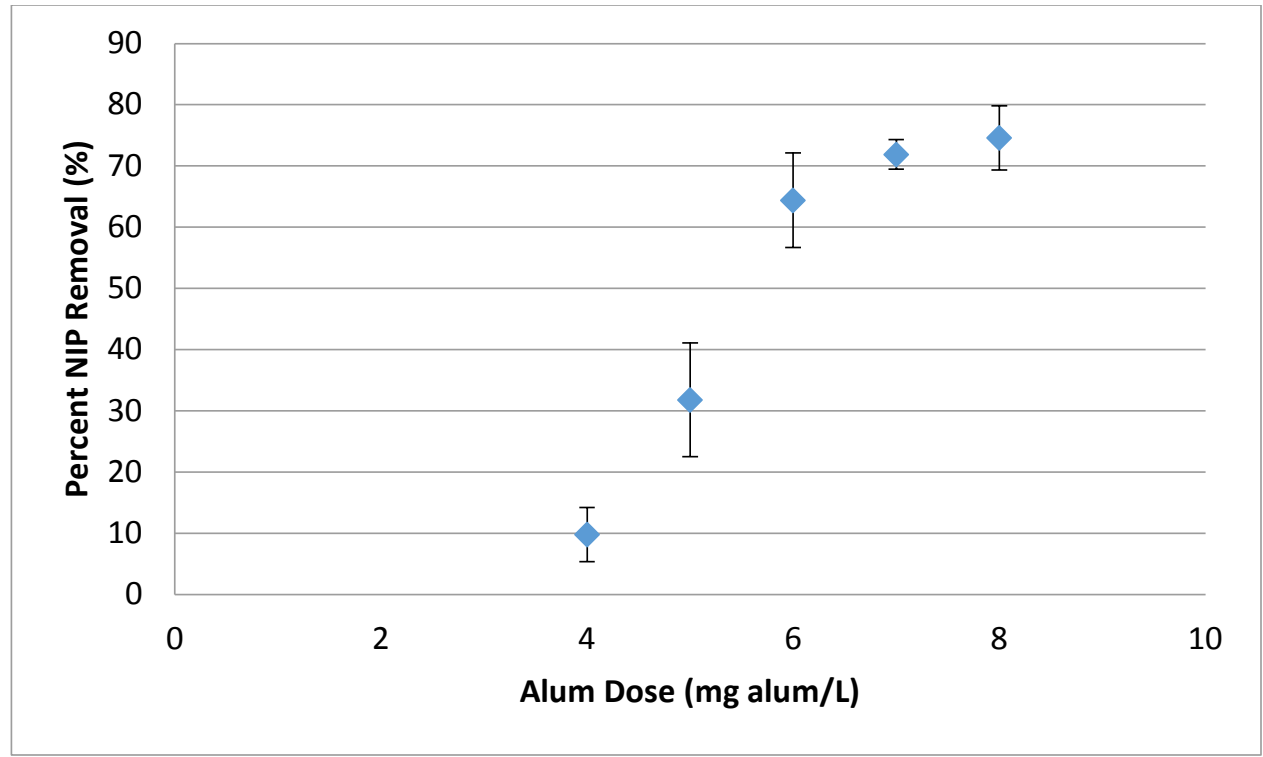

Figure 8-4(a):Percent removal of NIP in tap water by ballasted flocculation for varying alum doses based on turbidity measurements

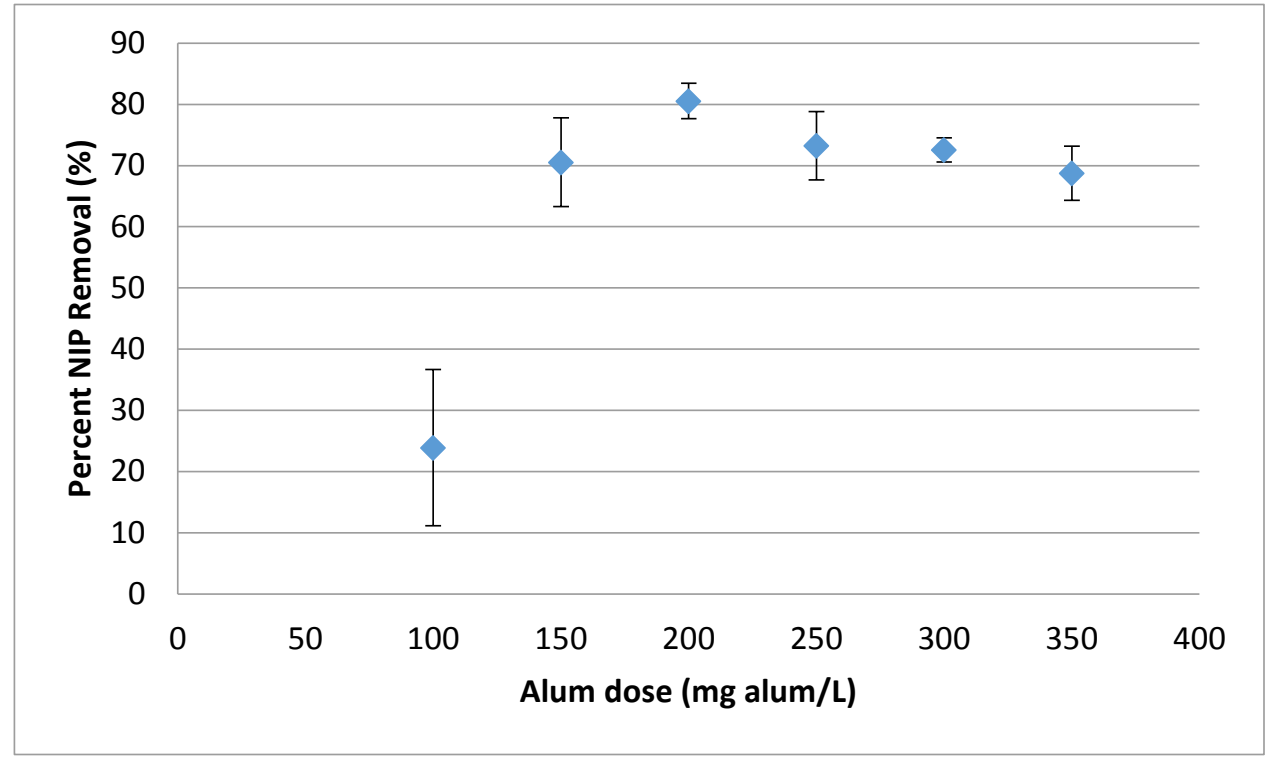

Figure 8-4 (b): Percent removal of NIP in wastewater by ballasted flocculation for varying alum doses based on turbidity measurements 
Increasing the alum dose also increased the efficiency of ballasted flocculation of NIP in wastewater. The factorial design experiments and related linear regression were not performed with wastewater Figure 8-4 b shows the effect of increasing alum dose on removal of NIP from secondary wastewater effluent. It should be noted that turbidity was used as a measure of the NIP concentration and since the wastewater did not have zero turbidity prior to the addition of the NIP, any reduction in the turbidity of the wastewater would also be attributed to a reduction in NIP concentration. The alum dose required for wastewater was much higher than that required for tap water due to the presence of organic matter and competing substances in the wastewater and a more concentrated stock solution was used to avoid dilution of samples. Figure 8-4 b shows a substantial increase in NIP removal caused by an increase in the alum dose from 100 to $200 \mathrm{mg} / \mathrm{L}$ alum from $24 \pm 13$ $\%$ to $81 \pm 3 \%$ followed by a slight decrease in removal for increasing alum doses up to 350 $\mathrm{mg} / \mathrm{L}$.

\subsubsection{Comparison of NIP removal processes}

\subsubsection{Comparison based on turbidity measurements}

Figure 8-5 provides a bar-graph comparison of the best-case scenarios for ballasted flocculation, filtration, and centrifugation as options for the removal of NIP from water samples following treatment as well as average scenarios for filtration and centrifugation, which were used to evaluate ballast flocculation as a pre-treatment. Since ballasted flocculation results in formation of larger flocs, a ballasted sand pre-treatment step was tested to investigate whether or not it could increase the efficiency of filtration. Since ballasted flocculation also increases the density of the flocs, it is possible that it could be 
used to decrease the centrifuge time or the centrifugal force required for NIP removal and was also evaluated as a potential pre-treatment for centrifugation. The optimized ballasted flocculation conditions were used prior to filtration with a $0.7 \mu \mathrm{m}$ pore size filter and centrifugation at $10,000 \mathrm{rpm}$ for 3 minutes. The results showed there was no significant improvement in filtration or centrifugation performance after pre-treatment with ballasted flocculation.

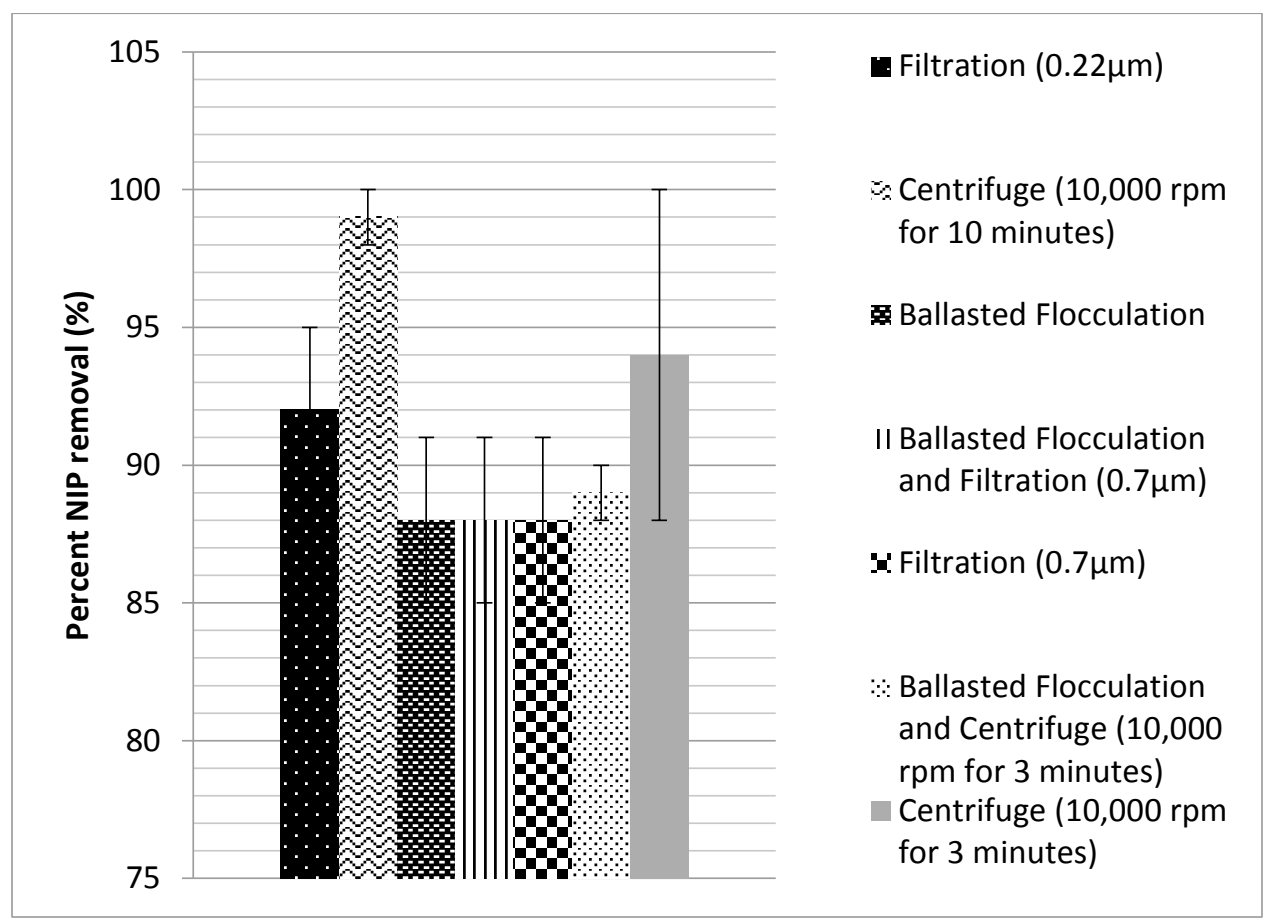

Figure 8-5: Comparison of percent removal of NIP for various removal methods based on turbidity measurements

To determine whether or not ballasted flocculation might provide a good pre-treatment option, the optimized ballasted flocculation conditions were used prior to filtration with a $0.7 \mu \mathrm{m}$ pore size and centrifugation at $10,000 \mathrm{rpm}$ for 3 minutes. Neither of these filtration 
or centrifugation conditions were the best-case scenarios. In the best-case scenarios for filtration and centrifugation, there was little room for improvement, so the effect of pretreatment would not have been measurable. The results of these treatments, without the pre-treatment are also shown in Figure 8-5. From the combined treatment results shown in Figure 8-5, there was no significant improvement provided by the pre-treatment.

An overall comparison of filtration, centrifugation, and ballasted flocculation either alone or as a pre-treatment, shows that centrifugation at $10,000 \mathrm{rpm}$ for 10 minutes provides the best treatment and filtration with a $0.22 \mu \mathrm{m}$ pore size filter was the second best treatment option. It should be noted that centrifugation is not commonly employed in municipal water and wastewater treatment and is limited to treating small flow volumes, such as those commonly seen for industrial wastewater treatment. Use of ballasted flocculation alone with gravity settling led to $88 \pm 3 \%$ NIP removal, but ballasted flocculation was not an effective pre-treatment prior to filtration or centrifugation.

The relative costs of treatment using the various NIP removal strategies also need to be considered. Although centrifugation provided the highest degree of removal, it is the most expensive and energy-intensive of the treatment methods studied and could only be used for small flowrates, such as those typically encountered during industrial wastewater treatment. Capital and operating costs vary depending on the specifics of treatment, but filtration or ballast flocculation would be much more feasible for the large flow volumes encountered in municipal water or wastewater treatment. 


\subsubsection{Comparison based on particle counter measurements}

In addition to turbidity measurements, particle count measurements were also considered to compare the performance of filtration (with a $0.22 \mu \mathrm{m}$ filter), centrifugation (at 10,000 rpm for 10 minutes) and ballasted flocculation in removing NIP. Results showing percent NIP removal based on particle count measurements are shown in Figure 8-6 a and b, respectively. Percent removal was calculated based on the difference in particle counts between the original and treated samples.

Figure 8-6 a shows the total particle count results for the $5 \mathrm{mg} / \mathrm{L}$ NIP in water before and after treatment by filtration, centrifugation, and ballasted flocculation. The results are averages of two experimental replicates consisting of three analytical replicates for each. The error bars represent the range of measured values. Figure 8-6 b provides the percent removal for each of the three treatments in comparison to the untreated sample. As

indicated in Figure 8-6 b, filtration achieved the highest degree of removal $(97 \pm 0.27 \%)$, while centrifugation and ballasted flocculation removed $39 \pm 4 \%$ and $44 \pm 9 \%$ of the particles, respectively. 


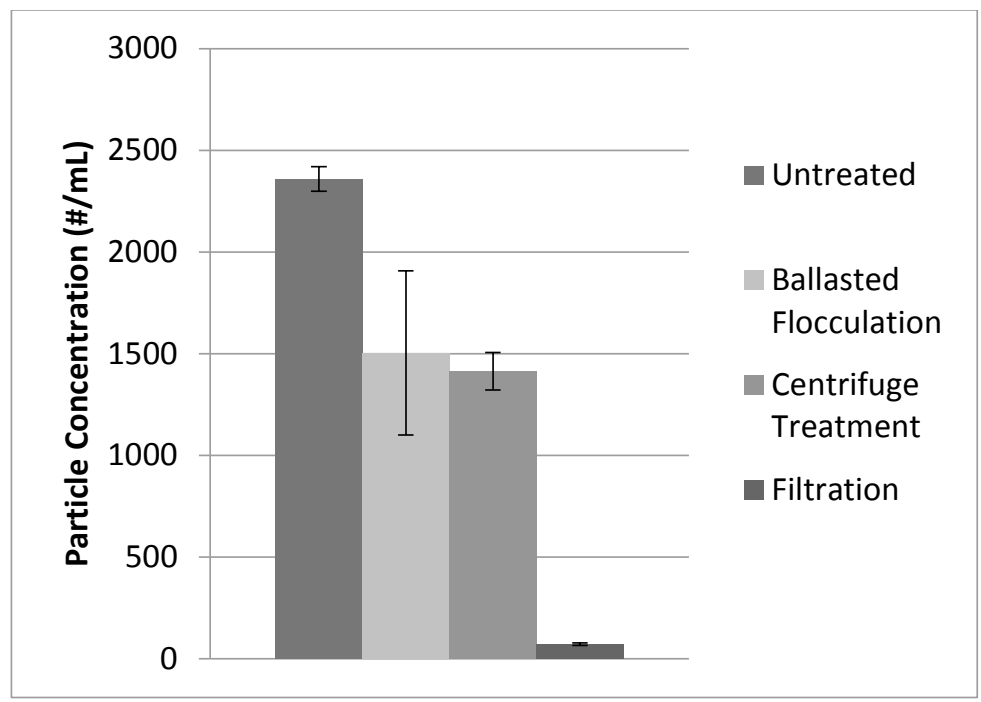

Figure 8-6(a): Particle count concentrations measured by the DPA 4100 Particle Analysis System before and after NIP removal for various removal methods

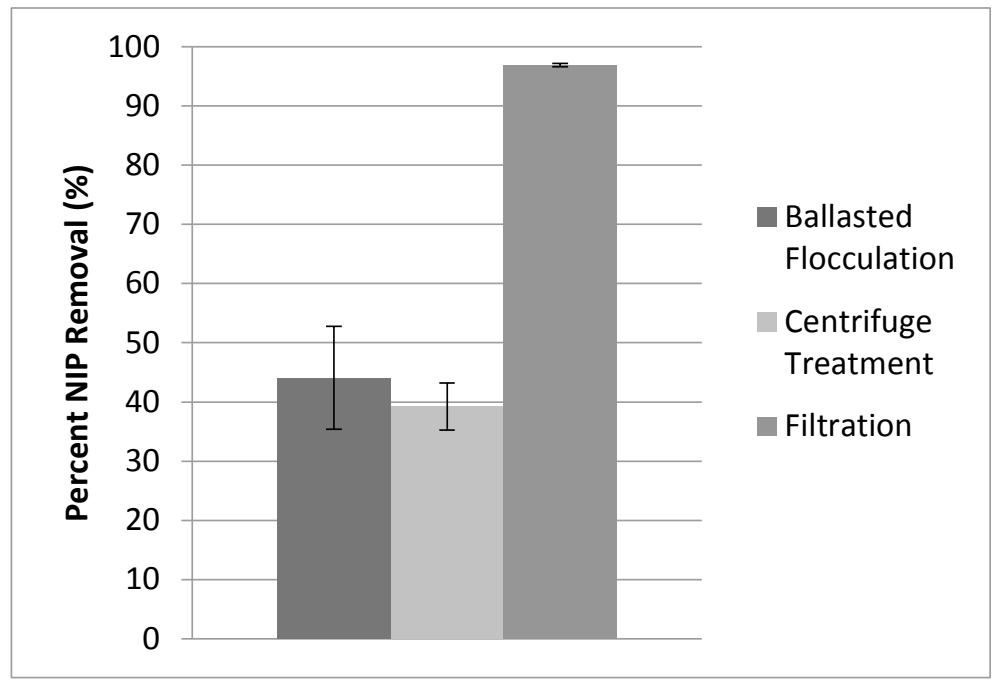

Figure 8-6 (b): Percent removal of NIP following removal with various removal methods based on particle concentrations measured by the DPA 4100 Particle Analysis System

A comparison between the results obtained with the particle counter, shown in Figure 8-6, and those obtained with turbidity measurements, shown in Figure 8-5, showed a large 
discrepancy between the turbidity and particle counter results for the centrifuge and ballasted flocculation treatments. The turbidity results shown in Figure 8-5 indicate $92 \pm$ $3 \%, 99 \pm 1 \%$, and $88 \pm 3 \%$ NIP removal for filtration $(0.22 \mu \mathrm{m})$, centrifuge treatment (10,000 rpm for 10 minutes), and ballasted flocculation, respectively.

The NIP particles had an average size of $333 \pm 76 \mathrm{~nm}$ (as measured from scanning electron microscope images), and measuring the concentration of very small particles in solution is difficult. The particle counter did not accurately count particles with diameters less than 2 $\mu \mathrm{m}$ (Thomas and Moore, 2004). Turbidimeters, on the other hand, are most sensitive for submicron particles with diameters close to the wavelength of visible light. The sensitivity of turbidity measurements is also related to the refractive index of the particles, and factors specific to the turbidimeter such as the wavelength and angle of light emitted. However, a study with latex particles of varying sizes found that the maximum sensitivity occurred at a wavelength of $0.2 \mu \mathrm{m}$. The sensitivity of turbidimeters declines for measuring large particles (several microns in size) which is why they are not necessarily considered sensitive for water treatment applications (Gregory, 1998). Therefore, although both particle counter and turbidity measurements are presented herein, turbidity provides a better measure of particle concentration for submicron particles such as NIP.

Results from the particle counter can be considered to refer to very large particles that are outliers in terms of their size. The filtration results using the particle counter $(97 \pm 0.27 \%)$ most closely matched those obtained with the turbidimeter $(92 \pm 3 \%)$ and in fact showed a slightly higher removal, perhaps because filtration preferentially removes larger particles. 
The filter paper had a pore diameter of $0.22 \mu \mathrm{m}$ and thus can be expected to remove particles greater than $0.22 \mu \mathrm{m}$ in diameter. The low percent removal values for the centrifuge and ballasted flocculation measured using the particle counter may have also occurred due to the formation of agglomerates of particles during treatment, which were not subsequently removed and contributed to an increase in the number of larger particles, leading to a falsely low measure for the removal of very large particles. Larger agglomerates of particles would be more easily removed than smaller particles, but since smaller particles were not measurable, agglomerates of small particles not large enough to be removed, but large enough to be measured would change the results.

\subsubsection{Evaluation of removal efficiency with different analytical devices}

The discrepancy between measurements obtained using the turbidimeter and particle counter to measure the efficiency of various methods for NIP removal highlights the need to better understand the behaviour of NIP particles in water, both before and after separation processes. SEM, zetasizer, and particle counter measurements were used to determine the particle size and distribution of NIP in its dry form as well as in water.

\subsubsection{Characterization of dry NIP}

Figure 8-7, below, shows a scanning electron microscope (SEM) image of dry NIP particles. From this image, the NIP had an average diameter of $333 \pm 76 \mathrm{~nm}$. The size of NIP particles is controlled by the polymerization method (Alexander, 2006). NIP are

spherical in shape and are densely packed in their solid form. To evaluate various treatment 
methods for removal of NIP from water, the NIP were sonicated in $1 \mathrm{~L}$ of tap water for 15 minutes to disperse the particles and then diluted to a final concentration of $5 \mathrm{mg} / \mathrm{L}$.

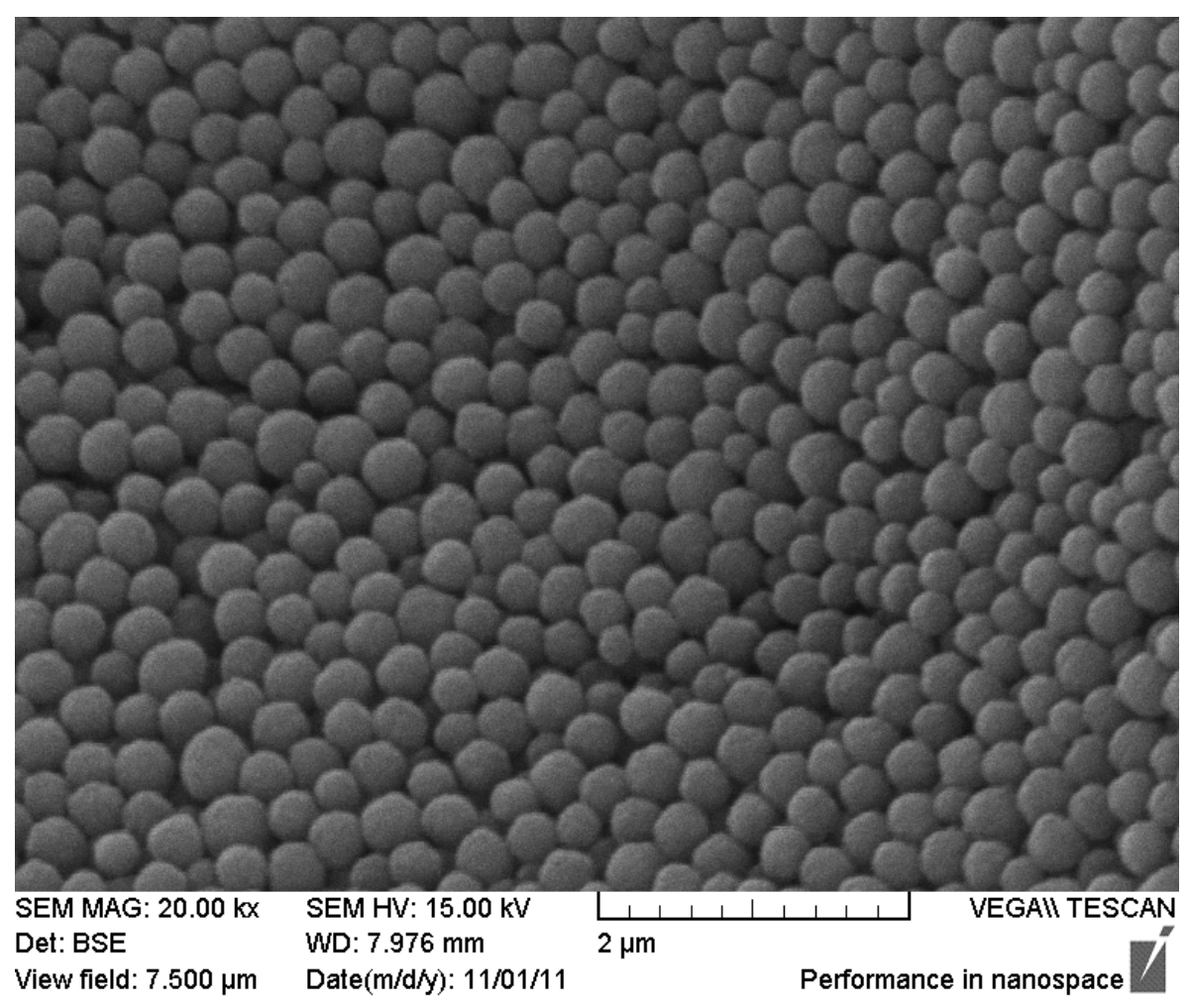

Figure 8-7: SEM image of NIP particles

For wastewater treatment purposes, it is important to determine whether NIP stay dispersed in water as single particles or as groups of aggregated particles. If they were present as groups of particles, the surface area available for adsorption of pollutants would be reduced; however, their removal would be easier. Therefore, it is necessary to establish an understanding of NIP behaviour in water, which can be drawn from an examination of the average particle sizes and particle size distributions both before and after treatment. 


\subsubsection{Characterization of NIP in water}

Particle size distributions of NIP in water were measured using both a zeta sizer (Malvern Nano ZS Zetasizer) and a particle counter (DPA 4100 Particle Analysis System). Zetasizer results show two analytical replicates. A second experimental replicate was also taken with the zetasizer, and results were used to calculate average particle size, but are not shown. The particle counter results are averages of two experimental replicates, each of which is an average of three analytical replicates and the error bars represent the difference between the experimental replicates.

Figure $8-8 \mathrm{a}$ and $\mathrm{b}$, provide particle size distributions of NIP in water $(5 \mathrm{mg} / \mathrm{L})$ obtained using the zetasizer (a) and particle counter (b). Figure 8-8 a shows the average particle size obtained using the Nano ZS Zetasizer. The $\mathrm{x}$-axis shows the equivalent circle diameter, which is the diameter of the particles, assuming that the particles are spherical. The NIP were spherical in shape, so this is an accurate assumption. It is important to note that the $\mathrm{x}$-axis is a logarithmic scale, so while the particle size distribution seen in Figure 8-8 a appears to be normal, it is skewed to the right. The y-axis shows the intensity of the signal for each diameter of particle, which provides a relative measurement of how many particles of each diameter are present. The average particle size prior to treatment as measured by the zetasizer was $217 \pm 4 \mathrm{~nm}$. This is smaller than the $333 \pm 76 \mathrm{~nm}$ particle size obtained from the SEM image. The zetasizer measures the hydrodynamic diameter, which is generally different from the geometric diameter. However, the zetasizer also averages a much larger sample size than the SEM, where the particle size was estimated from the average diameter of 5 particles, and thus the zetasizer may provide a better estimate. 
Nonetheless, these results indicate that the majority of the particles in solution were present as single particles rather than groups of particles. The average particle size in solution is not larger than that measured for single particles in powdered form, indicating that the majority of the particles measured by the zetasizer were single particles. However, measurements were not taken over an extended period of time, so the particles may form aggregates of particles over time.

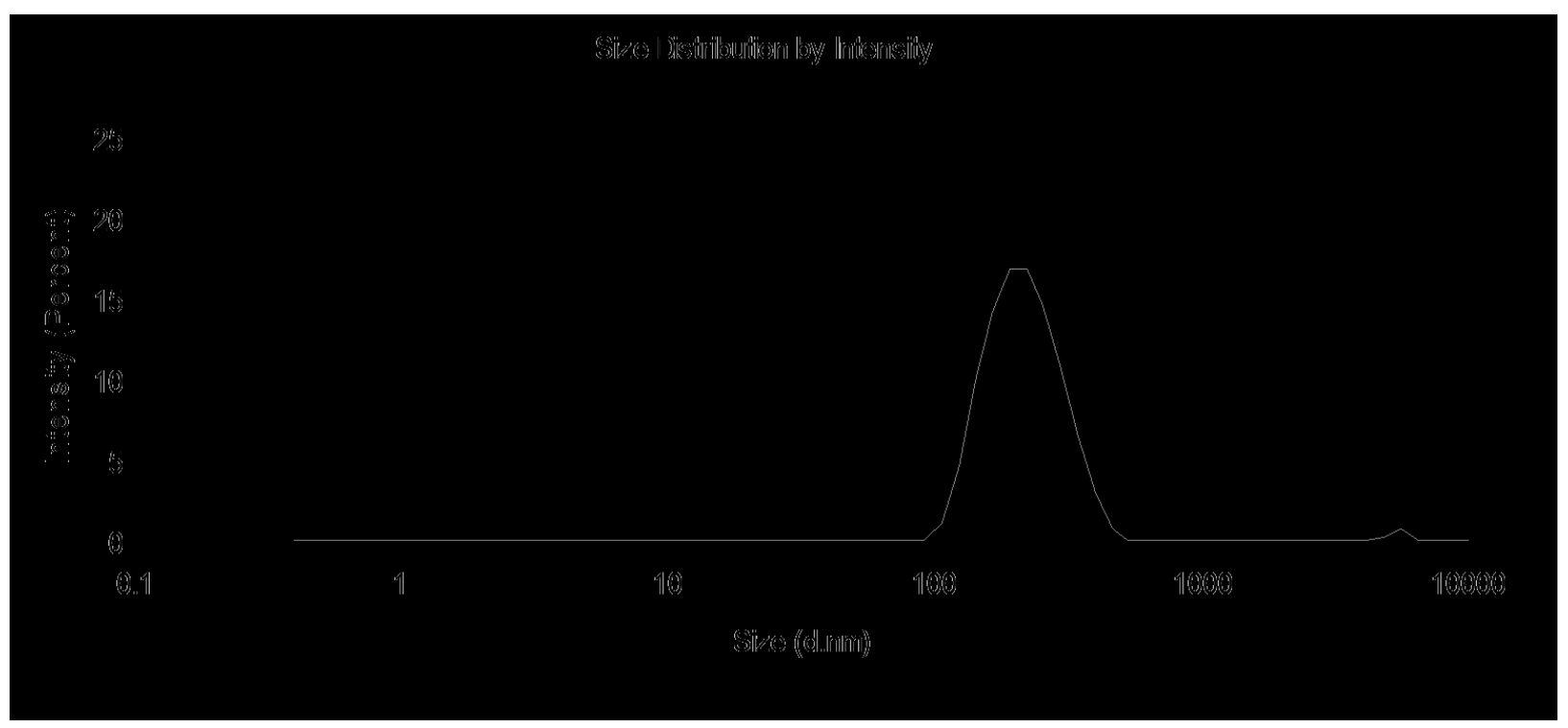

Figure 8-8 (a): NIP particle size distribution in water as measured by the Nano ZS Zetasizer 


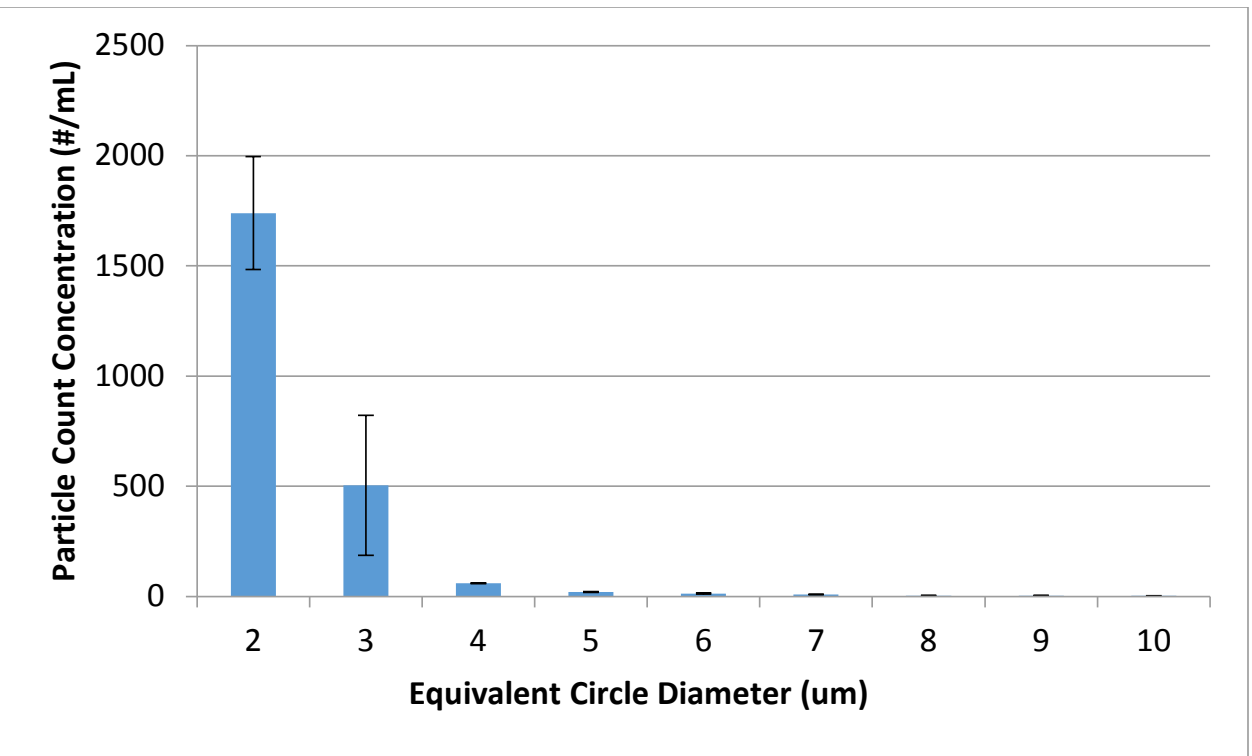

Figure 8-8 (b): NIP particle size distribution in water as measured by the DPA 4100 Particle Analysis System

The SEM image in Figure 8-7 indicates that the particles exhibited some spread in their average particle size. The average particle size measured from the SEM image had a spread of $76 \mathrm{~nm}$. The average particle diameter measured by the zetasizer has been given as 217 $\pm 4 \mathrm{~nm}$, and appears to indicate a smaller spread in particle size. However, the error indicated in this case refers to the standard deviation between average particle sizes measured from different replicates whereas the $76 \mathrm{~nm}$ from the SEM image refers to the standard deviation between measured particle sizes for single particles. This is better indicated by the curves shown in Figure 8-8 than the error indicated with the value of the average.

Figure $8-8$ b provides the particle size distribution obtained using the particle counter. The distribution shown is the average of two experimental replicates each of which was the 
average of three analytical replicates. The error bars represent the difference between the experimental replicates. The particle counter does not accurately count particles below 2 $\mu \mathrm{m}$ in diameter (Thomas and Moore, 2004). It is possible that some particles less than 2 $\mu \mathrm{m}$ were counted and grouped in the $2-3 \mu \mathrm{m}$ bin, but the particle counter is not a reliable method for counting sub-micron particles. Figure 8-8 b can be considered a close-up or detailed look at the very big NIP particles, or groups of particles, that were much larger than the average NIP size of $217 \pm 4 \mathrm{~nm}$. The particle counter was used in addition to the zeta sizer results, because, although the zetasizer was able to measure particles over the entire size range of NIP particles, it did not provide any information about the concentration of the particles. While the particle counter was able to measure concentrations of the larger particles only, this was information not available with the zeta sizer. Figure $8-8 \mathrm{~b}$, as expected, shows that the majority of these large particles were 2-3 $\mu \mathrm{m}$ in diameter, but a small number were up to $10 \mu \mathrm{m}$ in diameter.

Figure 8-9 a and b show NIP size distributions measured using the zetasizer and particle counter following centrifugation at 10,000 rpm for 10 minutes. Figure 8-9 a displays the distribution measured using the zetasizer. The average particle size following centrifuge treatment was $206 \pm 45 \mathrm{~nm}$. Again, the error here represents the difference between experimental replicates. Following centrifugation, there was a greater difference between experimental replicates than the untreated sample, presumably because there were more opportunities for differences to occur. In Figure 8-9 a it appears that the particle size distribution was skewed to the left. However, because the $\mathrm{x}$-axis scale is logarithmic, the skew was, in fact, to the right. A comparison of Figures 8-8 a (untreated) and 8-9 a seem 
to indicate a difference in the number of larger particles, and the spread of particle sizes is larger. This is potentially due to particles contacting each other and forming flocs during centrifuging.

Figure 8-9 b provides the particle size distribution obtained using the particle counter. As previously mentioned, the particle counter can only count very large particles $(>2 \mu \mathrm{m})$, but gives an indication of the behaviour of the larger particles. A comparison of Figures 88 (b) before NIP removal and 8-9 (b) indicates a clear change, with the centrifuged samples having more large particles. Again, this was probably due to aggregation of particles while being centrifuged.

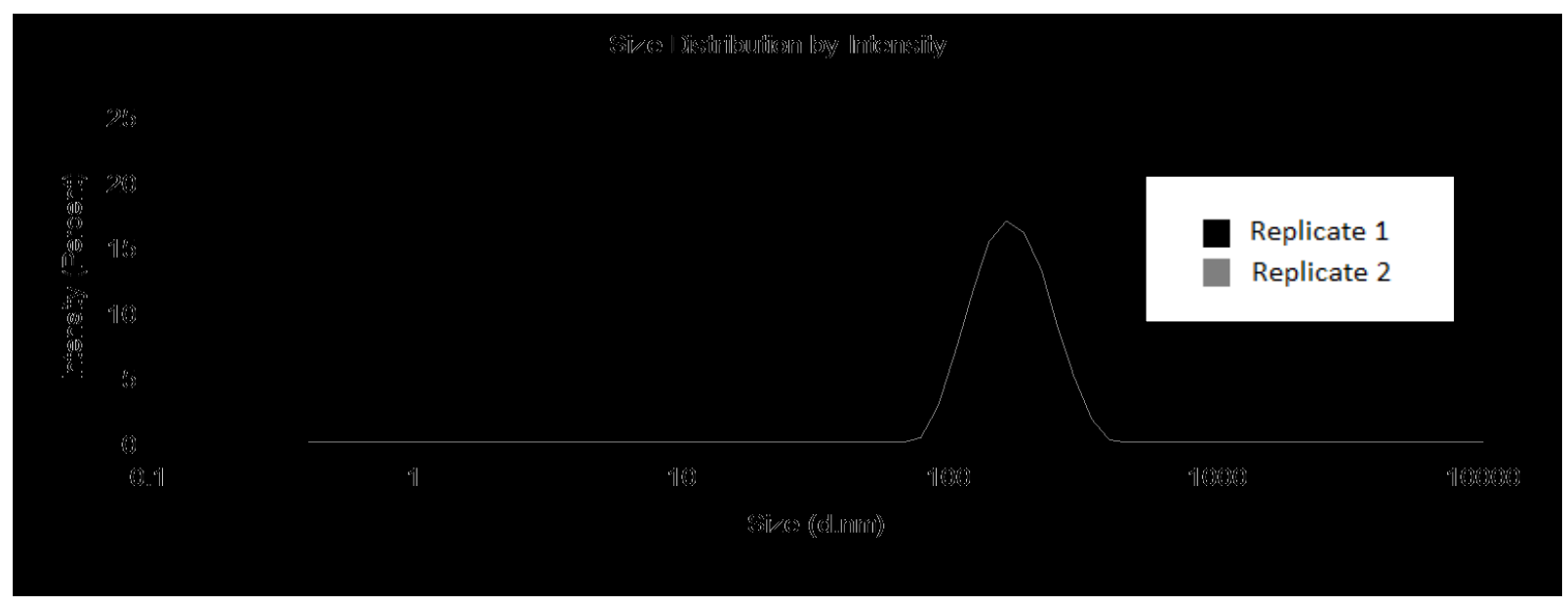

Figure 8-9 (a): NIP particle size distribution following centrifuge treatment as measured by the Nano ZS Zetasizer (2 replicates) 


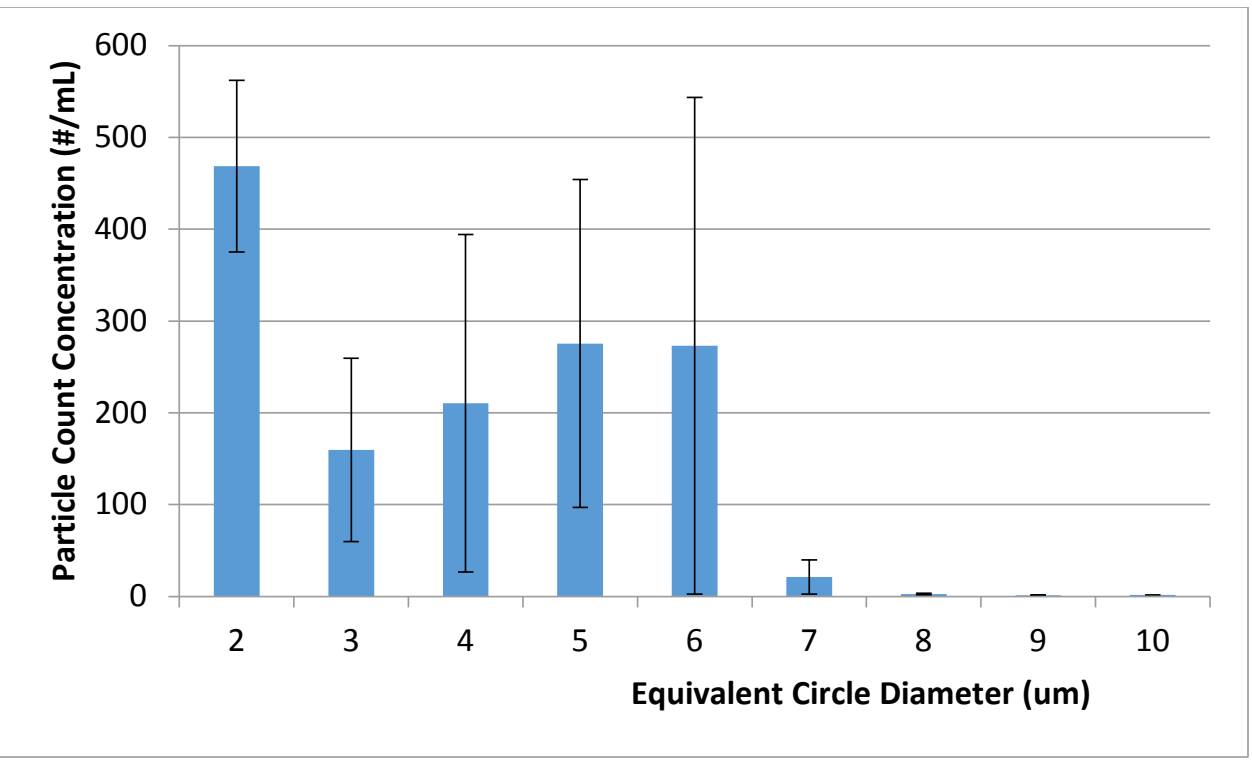

Figure 8-9 (b): NIP particle size distribution following centrifuge treatment as measured by the DPA 4100 Particle Analysis System

Figure 8-10 a and $\mathrm{b}$ shows the particle size distributions measured using the zetasizer and particle counter following filtration with a $0.22 \mu \mathrm{m}$ filter. Figure $8-10$ a presents the particle size distribution measured using the zetasizer. The average particle size following filtration was $275 \pm 70 \mathrm{~nm}$. Similar to the centrifuged samples, the filtered samples also showed greater differences than the untreated samples due to differences experienced during treatment. Although the average particle size remained within the range of error from the untreated sample, the average particle size for filtered samples can be expected to be larger than that of the untreated sample because filtration preferentially removes larger particles. Comparing the distribution shown in Figure 8-10 to that in Figure 8-8, the spread of particle sizes is much smaller and there are fewer larger particles. Figure 8-10 b shows the particle size distribution as measured by the particle counter following filtration. Compared to Figure 8-8 b, the number of large particles are fewer, but again, most of the particles are 2- 
$3 \mu \mathrm{m}$ in diameter. The particle count concentration for the filtered sample was also much lower than that for the unfiltered sample shown in Figure 8-8 b, illustrating that filtration effectively removed NIP from solution.

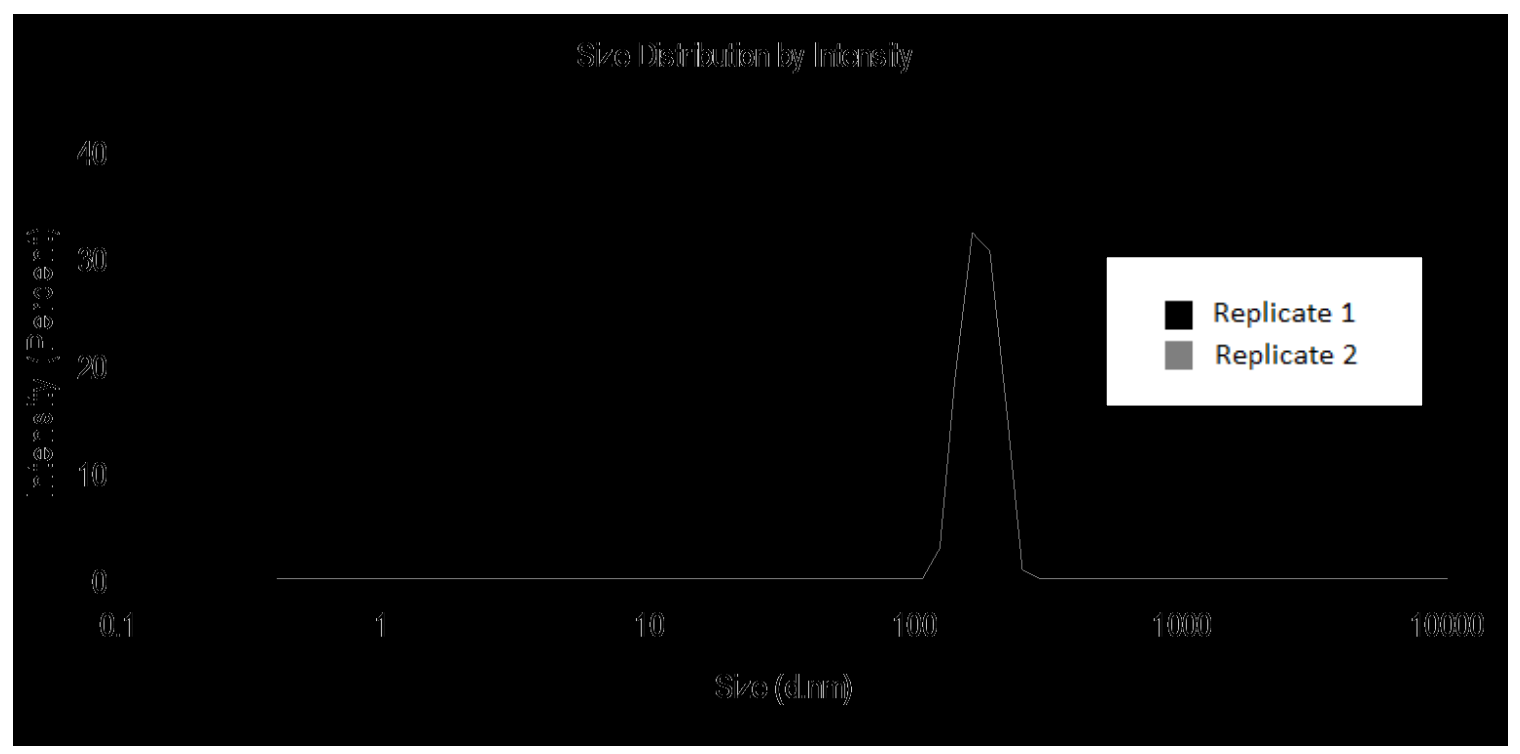

Figure 8-10 (a): NIP particle size distribution following filtration as measured by the Nano ZS Zetasizer (2 replicates)

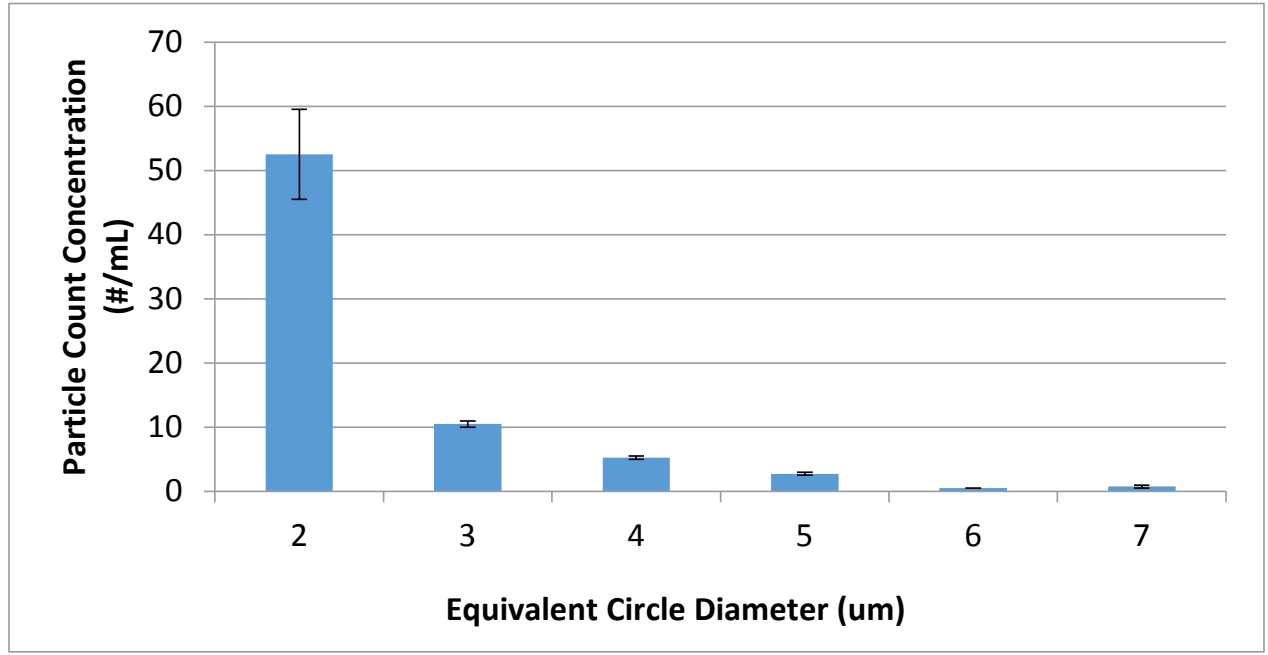


Figure 8-10 (b): NIP particle size distribution following filtration as measured by the DPA 4100 Particle Analysis System

Figure 8-11 $\mathrm{a}$ and $\mathrm{b}$ show the particle size distributions measured using the zetasizer and particle counter following ballasted flocculation. Figure 8-11 a shows the particle size distribution measured using the zetasizer. The average particle size following ballasted flocculation was $251 \pm 8 \mathrm{~nm}$, which was larger than that for the untreated sample $(217 \pm$ $4 \mathrm{~nm})$. This could be an indication of floc formation or it could be due to the presence of the ballasted sand. Comparing the distribution shown in Figure 8-11 to that in Figure 8-8, it is difficult to see a difference visually. Figure $8-11$ b shows the size distribution obtained with the particle counter, and also follows a similar pattern to that of the untreated sample in Figure $8-8 \mathrm{~b}$, with most of the large particles being $2 \mu \mathrm{m}$ in diameter. The difference between these two samples lies in the number of large particles.

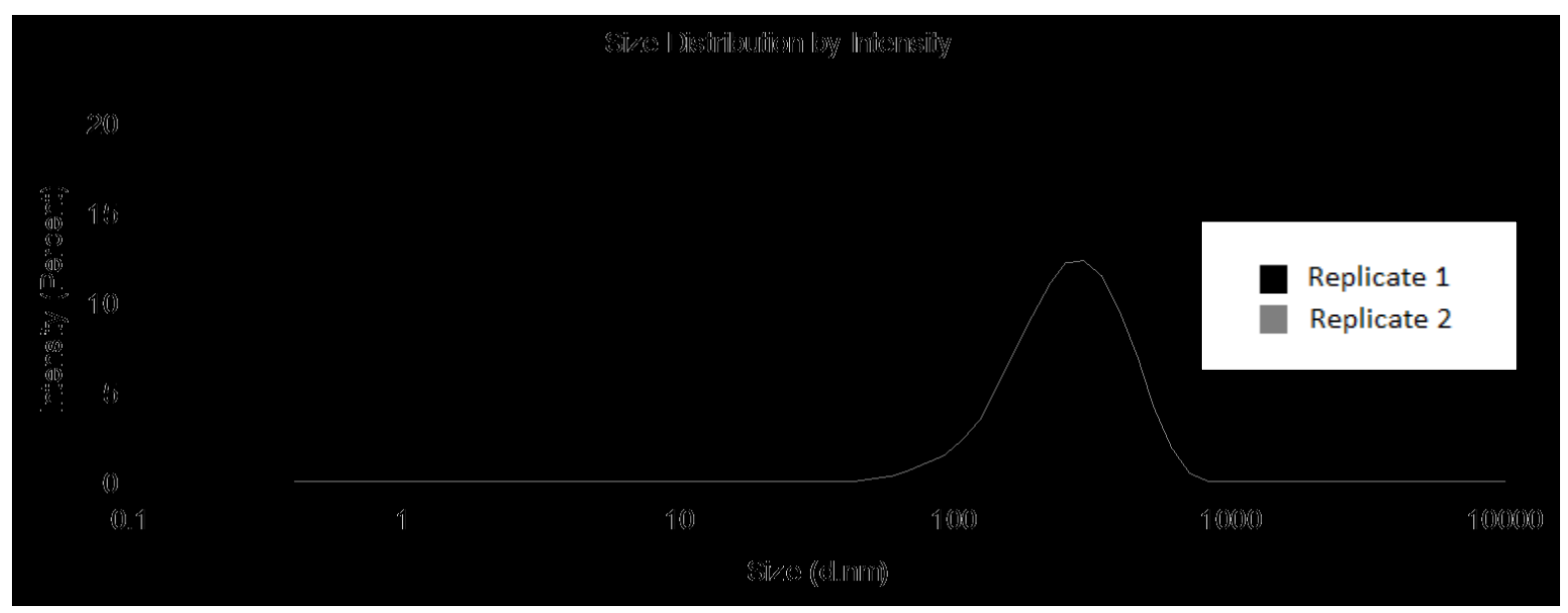

Figure 8-11 (a): NIP particle size distribution following ballasted flocculation as measured by the Nano ZS Zetasizer (2 replicates) 


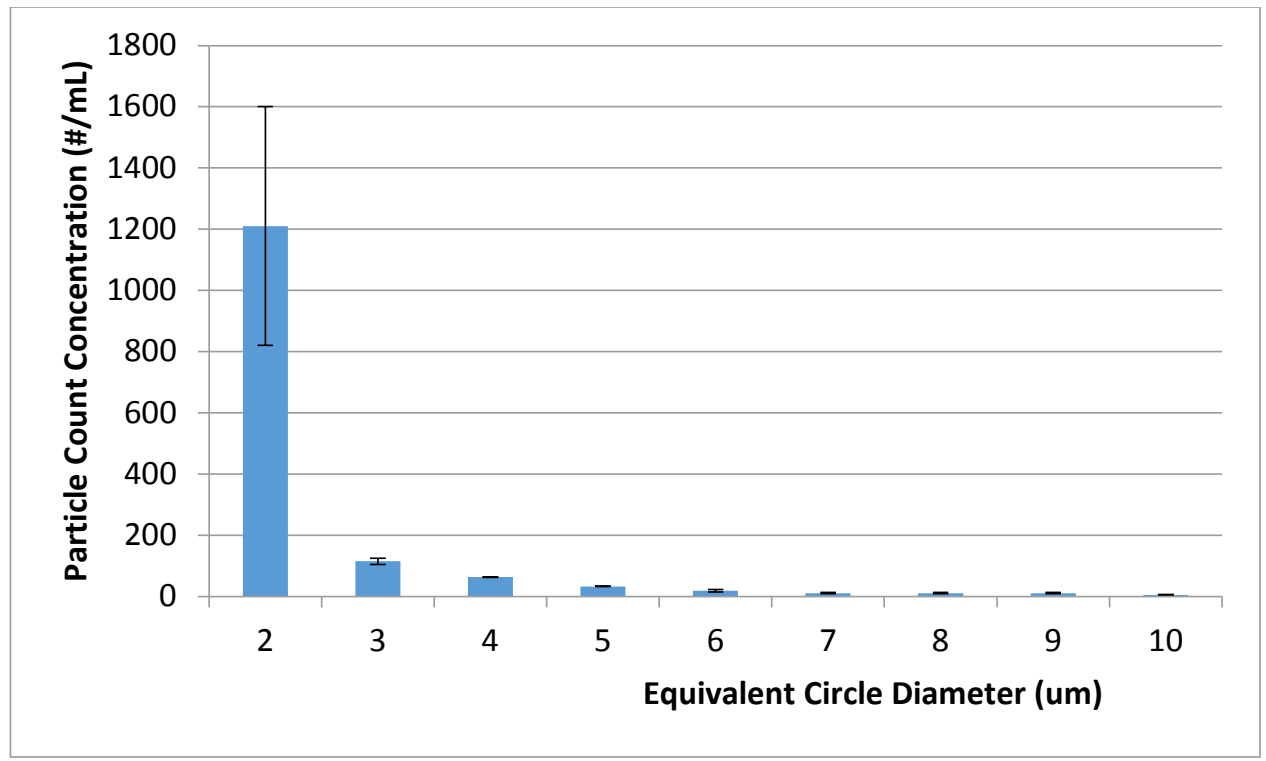

Figure 8-11(b): NIP particle size distribution following ballasted flocculation as measured by the DPA 4100 Particle Analysis System

Figure 8-12 summarizes the average particle sizes before and after treatment with centrifugation at $10,000 \mathrm{rpm}$ for 10 minutes, filtration with a $0.22 \mu \mathrm{m}$ filter, and ballasted flocculation, measured using the zetasizer. The results shown are averages of 2 experimental replicates and the error bars show the differences between the replicates. Each experimental replicate was calculated as the average between two analytical replicates. From Figure 8-12, there was a decrease in the average particle diameter following centrifuge treatment, and an increase following filtration. However, these changes were not bigger than the error associated with each of these measurements. The zeta sizer used dynamic light scattering, which tends to be skewed towards larger particles and there was some error inherent in this mode of measuring. There was an increase in the average particle size following ballasted flocculation, which was greater than the corresponding 
difference between measured samples. As previously stated, this difference was likely caused by either the presence of sand particles, or the formation of flocs of particles.

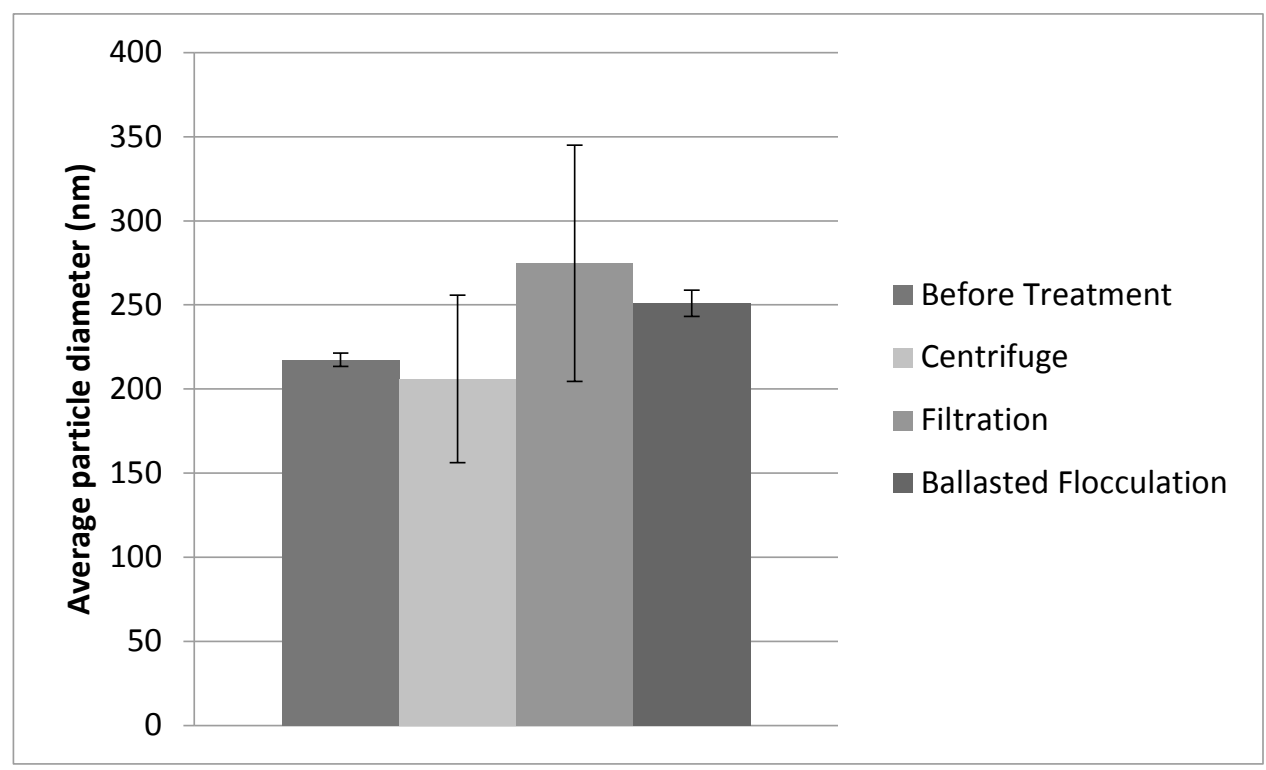

Figure 8-12: Average NIP particle sizes before and after various treatments as measured by the Nano ZS Zetasizer

\subsubsection{Exposure to acidic and basic conditions}

NIP can be exposed to a variety of $\mathrm{pH}$ conditions during treatment, so it was important to test them under a range of $\mathrm{pH}$ conditions. As a preliminary investigation into the ability of the NIP to withstand adverse conditions, SEM images of NIP were taken following $24 \mathrm{~h}$ of exposure to $\mathrm{pH}$ solutions ranging from 2 to 11 . Figure 8-7 shows a SEM image of NIP particles at a neutral $\mathrm{pH}$. Figure 8-13 a and $\mathrm{b}$ shows NIP particles that were exposed to $\mathrm{pH}$ 2 and 11, respectively. As shown in the figures, there were no visible changes to the NIP particles. A change was visible in the $\mathrm{pH} 11$ solution, which turned pink, indicating that there was some leaching from the particles. This did not occur in the $\mathrm{pH} 9$ solution. There 
was no visible change in NIP in the acidic solutions. These preliminary tests indicate that the NIP were stable over a range of $\mathrm{pH}$.

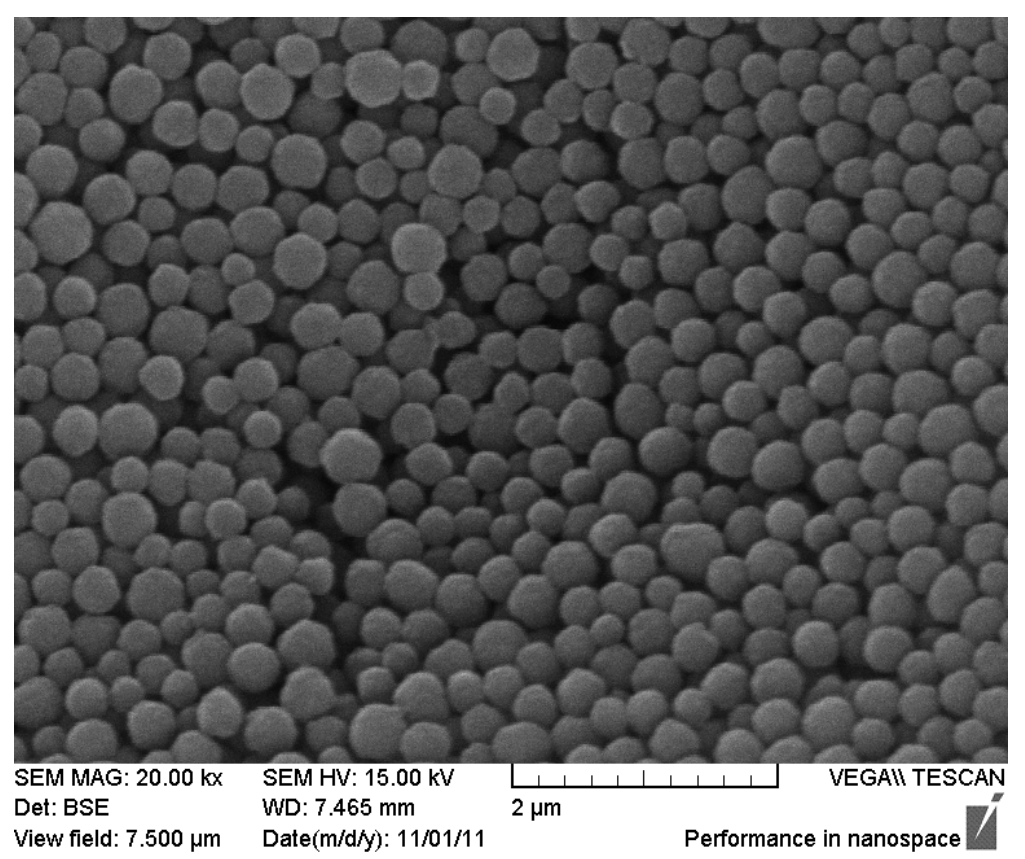

Figure 8-13 (a): SEM Image of NIP particles following 24 h exposure to $\mathrm{pH} 2$ (Acid) 


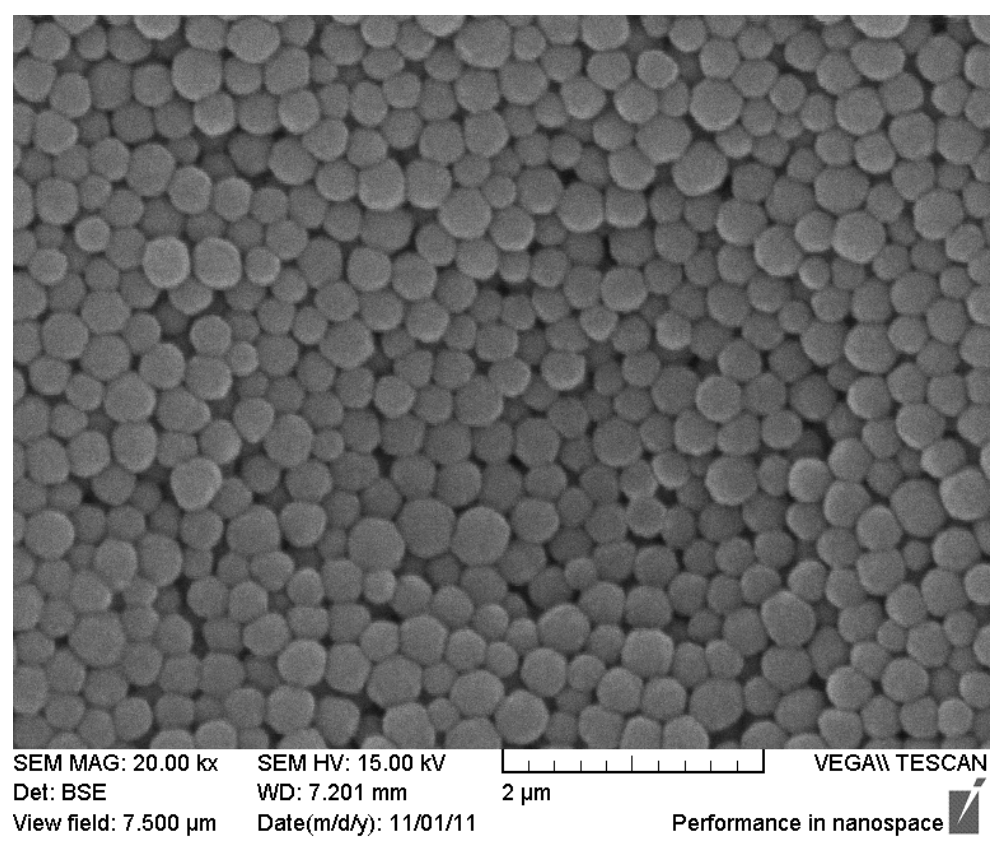

Figure 8-13 (b): SEM Image of NIP particles following 24 h exposure to pH 11 (Base)

\subsection{CONCLUSION}

MIP and NIP are used for removal of trace organic contaminants due in part to their small size which gives them a high surface area. The NIP were $333 \pm 76 \mathrm{~nm}$ in diameter, as estimated from SEM images. However, this small size also makes them difficult to remove following treatment. Use of filtration, centrifugation or ballasted flocculation following a batch application of the particles would take full advantage of the high surface area of the particles. Centrifuge treatment was capable of completely removing the particles $(99 \pm$ $1 \%$ ), and filtration with a $0.22 \mu \mathrm{m}$ syringe filter was able to remove most of the particles $(92 \pm 3 \%)$. Ballasted flocculation followed by gravity settling was able to remove $88 \pm 3 \%$ of the particles. These separation processes are already in use in many plants and use of conventional methods such as gravity settling may increase the flexibility of MIP and NIP 
allowing them to be applied in more diverse situations. The NIP were also found to exhibit no visible changes when exposed to solutions of $\mathrm{pH} 2-9$, which is an indication of their robustness.

\subsection{REFERENCES}

Braeken, L., and B. Van der Bruggen. 2009 Feasibility of Nanofiltration for the Removal of Endocrine Disrupting Compounds. Desalination, 240 (1-3), 127-131.

City of Ottawa, 2014. Drinking Water Quality Test Results Summary: Britannia Water Purification Plant. http://documents.ottawa.ca/en/node/6751

Deng, S., D. Shuai, Q. Yu, J. Huang, and G. Yu. 2009 Selective Sorption of Perfluorooctane Sulfonate on Molecularly Imprinted Polymer Adsorbents. Frontiers of Environmental Science \& Engineering in China, 3 (2), 171-177.

Desjardins, C., B. Koudjonou, and R. Desjardins. 2002 Laboratory Study of Ballasted Flocculation. Water Research, 36 (3), 744-54.

Fernández-Alvarez, P., M. Le Noir, and B. Guieysse. 2009 Removal and Destruction of Endocrine Disrupting Contaminants by Adsorption with Molecularly Imprinted Polymers Followed by Simultaneous Extraction and Phototreatment. Journal of Hazardous Materials, 163 (2-3), 1107-12.

Fuerhacker, M., A. Dürauer, and A. Jungbauer. 2001 Adsorption Isotherms of 17ßEstradiol on Granular Activated Carbon (GAC). Chemosphere, 44 (7), 15731579.

Gregory, J. 1998 Turbidity and Beyond. Filtration \& Separation, 35 (1), 63-67. 
Guo, W., W. Hu, J. Pan, H. Zhou, W. Guan, X. Wang, J. Dai, et al. 2011 Selective Adsorption and Separation of BPA from Aqueous Solution Using Novel Molecularly Imprinted Polymers Based on Kaolinite/Fe3O4 composites. Chemical Engineering Journal, 171 (2), 603-611.

Hajizadeh, S., H. Kirsebom, I. Y. Galaev, and B. Mattiasson. 2010 Evaluation of Selective Composite Cryogel for Bromate Removal from Drinking Water. Journal of Separation Science, 33 (12), 1752-9.

Krupadam, R. J., M. S. Khan, and S. R. Wate. 2010 Removal of Probable Human Carcinogenic Polycyclic Aromatic Hydrocarbons from Contaminated Water Using Molecularly Imprinted Polymer. Water Research, 44 (3), 681-8.

Le Noir, M., A.-S. Lepeuple, B. Guieysse, and B. Mattiasson. 2007a Selective Removal of 17Beta-Estradiol at Trace Concentration Using a Molecularly Imprinted Polymer. Water Research, 41 (12), 2825-31.

Le Noir, M., F. Plieva, T. Hey, B. Guieysse, and B. Mattiasson. 2007b Macroporous Molecularly Imprinted Polymer/Cryogel Composite Systems for the Removal of Endocrine Disrupting Trace Contaminants. Journal of Chromatography. A, 1154 (1-2), 158-64.

Li, Y., and E. P. C. Lai. 2010 Extraction of 17ß-Estradiol in Water Using Non-Imprinted Polymer Submicron Particles in Membrane Filters. Journal of Environmental Sciences, 22 (11), 1820-1825.

Li, Y., X. Li, J. Chu, C. Dong, J. Qi, and Y. Yuan. 2010 Synthesis of Core-Shell Magnetic Molecular Imprinted Polymer by the Surface RAFT Polymerization for the Fast and Selective Removal of Endocrine Disrupting Chemicals from 
Aqueous Solutions. Environmental Pollution (Barking, Essex : 1987), 158 (6), $2317-23$.

Li, Y., X. Li, Y. Li, J. Qi, J. Bian, and Y. Yuan. 2009 Selective Removal of 2,4Dichlorophenol from Contaminated Water Using Non-Covalent Imprinted Microspheres. Environmental Pollution (Barking, Essex : 1987), 157 (6), 1879_ 85.

Lin, Y., Y. Shi, M. Jiang, Y. Jin, Y. Peng, B. Lu, and K. Dai. 2008 Removal of Phenolic Estrogen Pollutants from Different Sources of Water Using Molecularly Imprinted Polymeric Microspheres. Environmental Pollution (Barking, Essex : 1987), 153 (2), 483-91.

Luo, X., Y. Zhan, Y. Huang, L. Yang, X. Tu, and S. Luo. 2011 Removal of WaterSoluble Acid Dyes from Water Environment Using a Novel Magnetic Molecularly Imprinted Polymer. Journal of Hazardous Materials, 187 (1-3), 27482.

Malvern Instruments. 2000 Dynamic Light Scattering: An Introduction in 30 Minutes. Http://Www.Malvern.Com/En/Products/Technology/Dynamic-Light-Scattering/, $1-8$.

Meng, Z., W. Chen, and A. Mulchandani. 2005 Removal of Estrogenic Pollutants from Contaminated Water Using Molecularly Imprinted Polymers. Environmental Science \& Technology, 39 (22), 8958-62.

Murray, A., B. Ormeci, and E. P. C. Lai. 2011 Removal of 17 Beta-Estradiol (E2) and its Chlorination By-Products from Water and Wastewater Using Non-Imprinted Polymer (NIP) Particles. Water Science and Technology, 64 (6), 1291-1297. 
Randhawa, M., I. Gartner, and C. Becker. 2007 Imprinted Polymers for Water Purification. Journal of Applied Polymer Science, 106 (5), 3321-3326.

Svenson, J., and I. a Nicholls. 2001 On the Thermal and Chemical Stability of Molecularly Imprinted Polymers. Analytica Chimica Acta, 435 (1), 19-24.

Thomas, D., and P. Moore. 2004 Water Treatment Plant Particle Characterization Using Digital Imaging Technology (April), 1-10.

Wei, S., A. Molinelli, and B. Mizaikoff. 2006 Molecularly Imprinted Micro and Nanospheres for the Selective Recognition of 17Beta-Estradiol. Biosensors \& Bioelectronics, 21 (10), 1943-51.

Xia, X., E. P. C. Lai, and B. Ormeci. 2012a Duo-Molecularly Imprinted Polymer-Coated Magnetic Particles for Class-Selective Removal of Endocrine-Disrupting Compounds from Aqueous Environment. Environmental Science and Pollution Research International,

Xia, X., E. P. C. Lai, and B. Ormeci. 2012b Ultrasonication Assisted Synthesis of Molecularly Imprinted Polymer Encapsulated Magnetic Nanoparticles for Rapid and Selective Removal of $17 \beta$ Estradiol from Aqueous Environment. Polymer Engineering \& Science, 52 (8), 1775-1783.

Xie, Y., H. Li, L. Wang, Q. Liu, Y. Shi, H. Zheng, M. Zhang, Y. Wu, B. Lu. 2011 Molecularly Imprinted Polymer Microspheres Enhanced Biodegradation of Bisphenol A by Acclimated Activated Sludge. Water Research, 45 (3), 1189-98. Yu, Q., S. Deng, and G. Yu. 2008 Selective Removal of Perfluorooctane Sulfonate from Aqueous Solution Using Chitosan-Based Molecularly Imprinted Polymer Adsorbents. Water Research, 42 (12), 3089-97. 
Zhongbo, Z., and J. Hu. 2008 Selective Removal of Estrogenic Compounds by Molecular Imprinted Polymer (MIP). Water Research, 42 (15), 4101-8. 


\section{CHAPTER 9: CONCLUSIONS AND FUTURE WORK}

The main conclusions of this study and areas for further study are presented below. More detailed conclusions are provided in the individual chapters.

The first question addressed by this research was if non-imprinted polymers (NIP) could be used to remove endocrine disrupting compounds (EDCs) and pharmaceuticals from water and wastewater. In Chapter 4, NIP were tested to evaluate their potential as a treatment for EDCs including: 17- $\beta$ estradiol (E2), $17-\alpha$ ethinylestradiol (EE2), estrone (E1), bisphenol A (BPA), and diethylstilbestrol (DES) as well as a group of 12 pharmaceuticals. NIP were able to remove $98 \%$ of spiked E2, $80 \%$ of EE2, $87 \%$ of BPA, and up to $97 \%$ of DES from single-solute solutions. In a mixed solutions with $0.5 \mathrm{ppm}$ each of E2, EE2, E1, BPA, and DES, percent removals with $500 \mathrm{mg} / \mathrm{L}$ of NIP were in the range of $24 \%$ (E2) to $49 \%$ (DES), and increased with increasing NIP concentrations, indicating that higher removal might be possible with higher concentrations of NIP. NIP were able to remove 9 out of 12 of the pharmaceuticals to some degree in deionized water and 3 out of 7 of the pharmaceuticals measured in wastewater. However, tests with pharmaceuticals were less conclusive due to low concentrations of pharmaceuticals. In conclusion, NIP are effective for removal of the EDCs studied, but further work is required to determine their effectiveness for adsorption of a variety of pharmaceutical compounds.

Future work required to more fully investigate the potential of NIP for water and wastewater treatment includes an investigation into the removal of a still wider range of EDCs and pharmaceuticals, to determine characteristics of emerging contaminants which 
make them suited to treatment with NIP. A wide range of contaminants of different classes and with different characteristics should be tested. More in-depth testing with pharmaceutical compounds can also be conducted to conclusively determine the ability of NIP to remove pharmaceuticals.

The second question addressed was whether NIP could be used to remove heavy metals from water and wastewater. NIP were tested for their ability to remove a $500 \mathrm{ppb}$ mixture of lead, copper, zinc, and nickel from both river water and wastewater. They were able to remove $82 \%$ of the lead, $46 \%$ of the copper, $55 \%$ of the zinc, and $17 \%$ of the nickel from a river water sample and $86 \%$ of the lead, $38 \%$ of the copper, $28 \%$ of the zinc, and $11 \%$ of the nickel from a wastewater sample. The presence of humic acid limited adsorption of heavy metals for both NIP and powder activated carbon. Isotherm tests were also conducted at $\mathrm{pH} 4$ in water, and displayed a linear relationship between heavy metal concentrations in solution and adsorbed heavy metal concentrations at equilibrium. Kinetics tests revealed that equilibrium was reached quickly, in less than 2 hours. Overall, NIP demonstrated excellent potential for adsorption of lead from natural waters, removing more than $82 \%$ of the lead with fast reaction kinetics, but were less effective in the presence of humic acid, and less effective for removal of other heavy metals.

Future work with heavy metals should focus on adsorption from solutions of varying $\mathrm{pH}$, because the results of this study indicated that adsorption was limited in acidic solutions. Effects of competition from humic acid should also be studied further, because adsorption was negligible in the presence of $20 \mathrm{mg} / \mathrm{L}$ of humic acid. Effects of ionic strength on heavy 
metal adsorption can also be studied in further experiments, because, if the NIP function through an ion exchange mechanism, the presence of other ions in solution may impact adsorption.

The third question addressed was how natural organic matter (NOM) competes with micropollutants (as modelled by methylene blue (MB) dye) for NIP adsorption sites, and how this compares to activated carbon. NIP were characterized along with three different powder activated carbons and found to have a much smaller surface area, and a smaller ratio of micropores to mesopores, which resulted in lower adsorption of MB from water. The NIP were then compared to Norit PAC 200 for adsorption of MB with competition from humic acid or wastewater. Competition occurred either during simultaneous exposure to the humic acid or wastewater and MB dye or pre-loading with humic acid or wastewater followed by subsequent adsorption of MB dye. Both the NIP and PAC 200 showed significant decreases of $27 \%$ for NIP $(\mathrm{p}=0.087)$ and $29 \%$ for PAC $200(\mathrm{p}=0.096)$ during simultaneous exposure to humic acid and methylene blue dye. Interestingly, there was no corresponding decrease, considering a 90\% confidence limit, for NIP or PAC 200 preloaded with humic acid and then exposed to MB. In fact, for PAC 200, the adsorption capacity of the activated carbon increased when it was pre-loaded with humic acid by $39 \%$ $(\mathrm{p}=0.0005)$. This may have been due to an increase in the negative surface charge of the activated carbon caused by adsorbed humic acid. For wastewater, the NIP showed no significant increase or decrease in adsorption capacity during either simultaneous exposure or pre-loading for a $90 \%$ confidence limit. The adsorption capacity of PAC 200 increased by $40 \%(\mathrm{p}=0.001)$ for simultaneous exposure to wastewater and MB. Pre-loading with 
wastewater had no effect on MB adsorption by PAC 200. Overall, the NIP and PAC 200 performed well in a variety of competitive situations with both humic acid and wastewater; however, it is not possible, at this point, to conclude whether or not NIP or PAC perform better under competition from humic acid or NOM in wastewater.

Competitive effects of humic acid on adsorption of MB were difficult to elucidate because humic acid and NOM from wastewater simultaneously affected both the available adsorption sites, and the surface charge on the adsorbents, cancelling each other. Future work should investigate methods for separating surface charge effects from competitive effects. Repeating experiments under a variety of $\mathrm{pH}$ conditions to control the surface charge, and measuring humic acid or NOM adsorbed in addition to MB adsorption may provide additional information.

The last question addressed how conventional methods such as filtration, centrifugation, and ballast flocculation could be used to remove NIP particles following treatment. Centrifugation and filtration with a $0.22 \mu \mathrm{m}$ filter were both very effective, removing 99 and $92 \%$ of the NIP, respectively. Ballast flocculation was less effective, removing only $88 \%$ of the NIP, and was not effective as a pre-treatment prior to centrifugation or filtration. Use of conventional methods such as gravity settling may increase the flexibility of MIP and NIP allowing them to be applied in more diverse situations, and centrifugation and filtration were effective for removal of NIP particles. 
Prior to full-scale application of NIP, further work is required to develop a feasible means of particle removal following adsorption. Centrifugation and filtration are both highly effective, but may not be feasible for treatment of large volumes of domestic wastewater. It may be possible to combine different removal strategies to develop a feasible and costeffective approach. NIP may also be more feasible for the treatment of smaller but highstrength wastewater flows such as industrial wastewater and side-streams at treatment plants. The overall feasibility of NIP for treatment of water or wastewater needs to be investigated for different scenarios including different flow volumes. Pilot scale testing is also required. In addition, the safety of both MIP and NIP particles should be evaluated due to the possibility of the particles escaping treatment and entering surface water bodies or drinking water supplies there have been no studies to date investigating the health or environmental effects of the particles. 


\section{APPENDIX A: REPORTING LIMITS FOR PHARMACEUTICALS}

\begin{tabular}{|l|l|}
\hline Analyte & Reporting Limit (ng/L) \\
\hline Acetaminophen & 59.9 \\
\hline Carbamazepine & 15.6 \\
\hline Cloxacillin & 1.56 \\
\hline Diphenhydramine & 10.4 \\
\hline Enrofloxacin & 0.623 \\
\hline Lincomycin & 3.12 \\
\hline Oxacillin & 3.12 \\
\hline Sulfadiazine & 1.56 \\
\hline Sulfamethizole & 0.821 \\
\hline Sulfanilamide & 15.6 \\
\hline Sulfathiazole & 1.56 \\
\hline
\end{tabular}




\section{APPENDIX B: WATER SAMPLE CHARACTERISTICS}

\section{B-1 WASTEWATER EFFLUENT CHARACTERISTICS (2012)}

\begin{tabular}{l} 
Average flow \\
Peak flow \\
\hline CBOD5 \\
\hline SS \\
\hline TP \\
\hline E coli (May 16 - Nov 15) \\
\hline
\end{tabular}

$$
\begin{aligned}
& 389,000 \mathrm{~m}^{3} / \text { day } \\
& 936,000 \mathrm{~m}^{3} / \text { day } \\
& 8 \mathrm{mg} / \mathrm{L} \\
& 12 \mathrm{mg} / \mathrm{L} \\
& 0.42 \mathrm{mg} / \mathrm{L} \\
& <200 \mathrm{CFU}
\end{aligned}
$$

(City of Ottawa, 2012)

\section{B-2 RIDEAU RIVER CHARACTERISTICS (HOGGS BACK 2012)}

\begin{tabular}{|l|l}
\hline Particulate residue & $5.8 \mathrm{mg} / \mathrm{L}$ \\
\hline Alkalinity & $143 \mathrm{mg} / \mathrm{L}$ \\
\hline pH & 8.12 \\
\hline Chloride & $35.6 \mathrm{mg} / \mathrm{L}$ \\
\hline Ammonium & $0.045 \mathrm{mg} / \mathrm{L}$ \\
\hline Total nitrogen & $0.52 \mathrm{mg} / \mathrm{L}$ \\
\hline Total phosphate & $0.021 \mathrm{mg} / \mathrm{L}$
\end{tabular}

(Province of Ontario, 2013)

\section{B-3 TAP WATER CHARACTERISTICS}

\begin{tabular}{|l|}
\hline Turbidity \\
\hline Total dissolved solids \\
\hline Alkalinity \\
\hline Total chlorine \\
\hline Total hardness \\
\hline
\end{tabular}

$0.04 \mathrm{NTU}$

$103 \mathrm{mg} / \mathrm{L}$

$33 \mathrm{mg} / \mathrm{L}$ as $\mathrm{CaCO}_{3}$

$1.99 \mathrm{mg} / \mathrm{L}$

$28.6 \mathrm{mg} / \mathrm{L}$ as $\mathrm{CaCO}_{3}$

(City of Ottawa, 2014) 
APPENDIX C: ACTIVATED CARBON INFORMATION SHEETS

САВOT

NORIT ACTIVATED CARBON

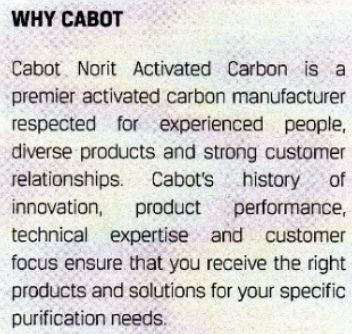

Cabot Norit Activated Carbon is a premier activated carbon manufacturer respected for experienced people, diverse products and strong customer relationships. Cabot's history of innovation, product performance, technical expertise and customer focus ensure that you receive the right products and solutions for your specific purification needs.

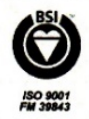

NORIT PAC 200 is a powdered activated carbon produced from select grades of coal. As a result of a unique patented activation process, this powdered activated carbon offers superior adsorption properties and is suited for the removal of taste, odor and color from water and industrial process applications. NORIT PAC 200 is Kosher certified and meets NSF/ANSI Standard 61 with a maximum use level of $250 \mathrm{mg} / \mathrm{L}$

\begin{tabular}{|c|c|c|}
\hline \multicolumn{3}{|c|}{ PRODUCT SPECIFICATIONS } \\
\hline lodine number & 900 min. & $\mathrm{mg} / \mathrm{g}$ \\
\hline Molasses number & 230 min. & \\
\hline Moisture & 3 max. & $\%$ as packed \\
\hline \multicolumn{3}{|l|}{ Mesh size (U.S. Sieve Series) } \\
\hline Less than 325 mesh $(45 \mu \mathrm{m})$ & $50 \mathrm{~min}$. & $\%$ \\
\hline \multicolumn{3}{|c|}{ TYPICAL PROPERTIES* } \\
\hline \multicolumn{3}{|l|}{ Mesh size (U.S. Sieve Series) } \\
\hline Less than 100 mesh $(150 \mu \mathrm{m})$ & 96 & $\%$ \\
\hline Less than 200 mesh $(75 \mu \mathrm{m})$ & 88 & $\%$ \\
\hline Food Chemical Codex & Passes & \\
\hline \multicolumn{3}{|c|}{ *For general information only, not to be used as purchase specifications. } \\
\hline \multicolumn{3}{|c|}{ PACKAGING/TRANSPORTATION } \\
\hline \multirow{2}{*}{\multicolumn{3}{|c|}{$\begin{array}{l}\text { Standard package is } 50 \mathrm{lb} \text { bags, } 45 \text { bags per pallet for a net pallet } \\
\text { weight of } 2250 \mathrm{lb} \text {. } \\
\text { Activated carbon (NOT REGULATED) }\end{array}$}} \\
\hline & & \\
\hline \multicolumn{3}{|c|}{ Exempt from DOT, IATA, and IMDG regulations } \\
\hline \multicolumn{3}{|c|}{ Import/Export classification: 3802.10 .0000 (HS Tariff Classification) } \\
\hline \multicolumn{3}{|c|}{ Domestic Freight Classification: NMFC 040560} \\
\hline CAS \# 7440-44-0 & & \\
\hline
\end{tabular}

\section{CABOT}

\section{NORT ACTVAREO CARBO}




\section{САВOT}

NORIT ACTIVATED CARBON

\section{DARCO ${ }^{\circledR} \mathrm{KB}-\mathrm{M}$}

\section{WHY CABOT}

Cabot Norit Activated Carbon is a premier activated carbon manufacturer respected for experienced people diverse products and strong customer relationships. Cabot's history of innovation, product performance, technical expertise and customer focus ensure that you receive the right products and solutions for your specific purification needs.

\section{POWDERED ACTIVATED CARBON}

DARCO KB-M is a powdered activated carbon produced by chemical activation of wood under carefully controlled conditions using the phosphoric acid process. It has exceptional adsorptive properties for a wide range of applications particularly in the chemical and food industries. DARCO KB-M is especially effective in adsorbing high molecular weight organics such as proteins and large color bodies. DARCO KB-M is Kosher certified and meets NSF/ANSI Standard 61.

\begin{tabular}{|c|c|c|}
\hline \multicolumn{3}{|c|}{ SPECIFICATIONS } \\
\hline Molasses decolorizing efficiency & 160 min. & as packed \\
\hline Iron, Zacher method & 175 max. & $\mathrm{ppm}$ as $\mathrm{Fe}$ \\
\hline Moisture & $12 \max$ & $\%$ as packed \\
\hline $\mathrm{pH}$ & 2.0 to 4.0 & \\
\hline \multicolumn{3}{|c|}{ TYPICAL PROPERTIES* } \\
\hline lodine number & 800 & $\mathrm{mg} / \mathrm{g}$ \\
\hline Methylene blue adsorption & 23 & $\mathrm{~g} / 100 \mathrm{~g}$ \\
\hline \multirow[t]{2}{*}{ Apparent density, tamped } & 0.38 & $\mathrm{~g} / \mathrm{mL}$ \\
\hline & 24 & $\mathrm{lb} / \mathrm{ft}^{3}$ \\
\hline Ash & 5 & mass-\% \\
\hline \multicolumn{3}{|l|}{ Particle size, laser } \\
\hline d5 & 4 & $\mu \mathrm{m}$ \\
\hline d50 & 25 & $\mu \mathrm{m}$ \\
\hline d95 & 66 & $\mu \mathrm{m}$ \\
\hline Food Chemical Codex & Passes & \\
\hline \multicolumn{3}{|c|}{ *For general information only, not to be used as purchase specifications. } \\
\hline \multicolumn{3}{|c|}{ PACKAGING/TRANSPORTATION } \\
\hline \multicolumn{3}{|c|}{$\begin{array}{l}\text { Standard package is } 40 \mathrm{lb} \text { bags, } 25 \text { bags per pallet for a net pallet weight } \\
\text { of } 1000 \mathrm{lbs} \text {. }\end{array}$} \\
\hline \multicolumn{3}{|c|}{ Carbon, activated, 4.2, UN1362, PGIII } \\
\hline \multicolumn{3}{|c|}{ Import/Export classification: 3802.10 .0000 (HS Tariff Classification) } \\
\hline \multicolumn{3}{|c|}{ Domestic Freight Classification: NMFC 040560} \\
\hline CAS \# 7440-44-0 & & \\
\hline
\end{tabular}

\section{CABOT

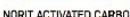




\section{САВOT}

NORIT ACTIVATED CARBON

DARCO ${ }^{\circledR} \mathrm{S}-51$

\section{WHY САВOT}

Cabot Norit Activated Carbon is a premier activated carbon manufacturer respected for experienced people, diverse products and strong customer relationships. Cabot's history of innovation, product performance, technical expertise and custome focus ensure that you receive the right products and solutions for your specific purification needs.

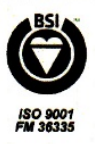

\section{POWDERED ACTIVATED CARBON}

DARCO S-51 is an acid washed powdered activated carbon produced by steam activation of lignite coal. It has high adsorptive capacity, good filterability, and above average purity. DARCO $\mathbf{5 - 5 1}$ is particularly suited for many purification applications such as treatment of sugar solutions, corn syrups, and fine chemicals. DARCO S-51 is Kosher certified and meets NSF/ANSI Standard 61.

\begin{tabular}{|c|c|c|}
\hline \multicolumn{3}{|c|}{ PRODUCT SPECIFICATIONS } \\
\hline Molasses decolorizing efficiency & $95 \mathrm{~min}$. & \\
\hline Moisture & 10 max. & $\%$ as packed \\
\hline $\mathrm{pH}$, water extract & 4.3 to 7.0 & \\
\hline \multicolumn{3}{|l|}{ Particle size, laser } \\
\hline d5 & $2.7 \mathrm{~min}$. & $\mu \mathrm{m}$ \\
\hline d50 & $30-42$ & $\mu \mathrm{m}$ \\
\hline d95 & 130 max. & $\mu \mathrm{m}$ \\
\hline \multicolumn{3}{|c|}{ TYPICAL PROPERTIES* } \\
\hline Iron, Zacher method & 250 & $\mathrm{ppm}$ as $\mathrm{Fe}$ \\
\hline Water solubles & 1.0 & $\%$ \\
\hline \multirow[t]{2}{*}{ Bulk density, tamped } & 0.51 & $\mathrm{~g} / \mathrm{mL}$ \\
\hline & 32 & $\mathrm{lb} / \mathrm{ft}^{3}$ \\
\hline Surface area & 650 & $\mathrm{~m}^{2} / \mathrm{g}$ \\
\hline Total pore volume & 1.0 & $\mathrm{~mL} / \mathrm{g}$ \\
\hline Food Chemical Codex & Passes & \\
\hline \multicolumn{3}{|c|}{ *For general information only, not to be used as purchase specifications. } \\
\hline \multicolumn{3}{|c|}{ PACKAGING/TRANSPORTATION } \\
\hline \multicolumn{3}{|l|}{ Standard packages: } \\
\hline \multicolumn{3}{|c|}{$40 \mathrm{lb}$ paper bag, 50 bags per pallet for a net pallet weight of $2000 \mathrm{lb}$} \\
\hline \multicolumn{3}{|c|}{$44.1 \mathrm{lb}$ paper bag, 50 bags per pallet for a net pallet weight of $2205 \mathrm{lb}$} \\
\hline \multirow{2}{*}{\multicolumn{3}{|c|}{$\begin{array}{l}\text { Woven polypropylene bulk bag with a plastic liner } 900 \text { or } 1200 \mathrm{lb} \text { net } \\
\text { Activated carbon (NOT REGULATED) }\end{array}$}} \\
\hline & & \\
\hline \multicolumn{3}{|c|}{ Exempt from DOT, IATA, and IMDG regulations } \\
\hline \multicolumn{3}{|c|}{ Import/Export classification: 3802.10 .0000 (HS Tariff Classification) } \\
\hline \multicolumn{3}{|c|}{ Domestic Freight Classification: NMFC 040560} \\
\hline CAS \# 7440-44-0 & & \\
\hline
\end{tabular}

\section{CABOT}




\section{APPENDIX D: SUWANNEE RIVER HUMIC ACID STANDARD (II) DATA PROVIDED BY THE INTERNATIONAL HUMIC SUBSTANCES SOCIETY}

\begin{tabular}{|l|l|l|l|l|l|l|l|l|l|}
\hline \multicolumn{1}{|l|}{ Chemical Characterization (International Humic Substances Society, 2015a) } \\
\hline $\mathrm{C}(\%)$ & $\mathrm{H}(\%)$ & $\mathrm{O}(\%)$ & $\begin{array}{l}\mathrm{N} \\
(\%)\end{array}$ & $\begin{array}{l}\mathrm{S} \\
(\%)\end{array}$ & $\mathrm{P}(\%)$ & & & \\
\hline 52.63 & 4.28 & 42.04 & 1.17 & 0.54 & 0.013 & & & & \\
\hline Functional Groups (International Humic Substances Society, 2015b) ** & \\
\hline Carboxyl & Phenolic & $\mathrm{Q}_{1}$ & $\begin{array}{l}\text { Log } \\
\mathrm{k}_{1}\end{array}$ & $\mathrm{n}_{1}$ & $\mathrm{Q}_{2}$ & $\begin{array}{l}\text { Log } \\
\mathrm{k}_{2}\end{array}$ & $\mathrm{n}_{2}$ & $\mathrm{~N}$ & $\mathrm{RMSE}$ \\
\hline 9.13 & 3.72 & 9.74 & 4.35 & 3.30 & 4.48 & 10.44 & 1.73 & 171 & 0.0815 \\
\hline
\end{tabular}

** The carboxyl functional groups are represented by the charge density $(\mathrm{meq} / \mathrm{gC})$ at $\mathrm{pH} 8$. The phenolic functional groups are measured by the change in charge density between $\mathrm{pH} 8$ and $10 . \mathrm{Q}_{1}$ and $\mathrm{Q}_{2}$ are the maximum charge densities of the carboxyl and phenolic functional groups, respectively, and $\log \mathrm{k}_{1}$ and $\log \mathrm{k}_{2}$ are the mean $\log \mathrm{k}$ values for binding of protons. The empirical parameters, $\mathrm{n}_{1}$ and $\mathrm{n}_{2}$, describe the width of the variability of the Log $\mathrm{k}$ values. $\mathrm{N}$ is the number of points used for the titration, and RMSE is the root of the mean square error for fitting the data to a Langmuir-Freundlich model. 
APPENDIX E: PARAMETER TESTING

\begin{tabular}{|l|l|l|l|l|}
\hline $\begin{array}{l}\text { E2 concentration } \\
(\mathrm{ppb})\end{array}$ & Batch & $\begin{array}{l}\text { Particle Mass } \\
(\mathrm{mg} / \mathrm{mL})\end{array}$ & Time & $\begin{array}{l}\text { percent } \\
\text { removal }\end{array}$ \\
\hline 2 & 1 & 5 & 5 & 99.93 \\
\hline 6 & 1 & 5 & 5 & 99.79 \\
\hline 10 & 1 & 5 & 5 & 99.89 \\
\hline 14 & 1 & 5 & 5 & 99.91 \\
\hline 2 & 1 & 10 & 5 & 99.36 \\
\hline 6 & 1 & 10 & 5 & 99.75 \\
\hline 10 & 1 & 10 & 5 & 99.85 \\
\hline 14 & 1 & 10 & 5 & 99.93 \\
\hline 2 & 1 & 15 & 5 & 99.80 \\
\hline 6 & 1 & 15 & 5 & 99.85 \\
\hline 10 & 1 & 15 & 5 & 99.94 \\
\hline 14 & 1 & 15 & 5 & 99.95 \\
\hline 2 & 1 & 20 & 5 & 99.73 \\
\hline 6 & 1 & 20 & 5 & 99.87 \\
\hline 10 & 1 & 20 & 5 & 99.93 \\
\hline 14 & 1 & 20 & 5 & 99.92 \\
\hline 2 & 1 & 5 & 7.5 & 99.43 \\
\hline 6 & 1 & 5 & 7.5 & 99.77 \\
\hline 10 & 1 & 5 & 7.5 & 99.82 \\
\hline 14 & 1 & 5 & 7.5 & 99.66 \\
\hline 2 & 1 & 10 & 7.5 & 99.75 \\
\hline 6 & 1 & 10 & 7.5 & 99.86 \\
\hline 10 & 1 & 10 & 7.5 & 99.77 \\
\hline 14 & 1 & 10 & 7.5 & 99.84 \\
\hline 2 & 1 & 15 & 7.5 & 99.71 \\
\hline 6 & 1 & 15 & 7.5 & 99.70 \\
\hline 10 & 1 & 15 & 7.5 & 99.90 \\
\hline 14 & 1 & 15 & 7.5 & 99.84 \\
\hline 6 & 1 & 20 & 7.5 & 99.75 \\
\hline 10 & 1 & 20 & 7.5 & 99.91 \\
\hline 14 & 1 & 20 & 7.5 & 99.88 \\
\hline 2 & 1 & 20 & 7.5 & 99.89 \\
\hline 6 & 1 & 5 & 10 & 99.73 \\
\hline 10 & 1 & 5 & 10 & 99.78 \\
\hline 14 & 1 & 5 & 10 & 99.91 \\
\hline & 1 & 5 & 10 & 99.87 \\
\hline 6 & & & & \\
\hline 6 & 15 & 5 & 5 & 5 \\
\hline
\end{tabular}




\begin{tabular}{|c|c|c|c|c|}
\hline 2 & 1 & 10 & 10 & 99.75 \\
\hline 6 & 1 & 10 & 10 & 99.86 \\
\hline 10 & 1 & 10 & 10 & 99.91 \\
\hline 14 & 1 & 10 & 10 & 99.94 \\
\hline 2 & 1 & 15 & 10 & 99.75 \\
\hline 6 & 1 & 15 & 10 & 99.88 \\
\hline 10 & 1 & 15 & 10 & 99.95 \\
\hline 14 & 1 & 15 & 10 & 99.96 \\
\hline 2 & 1 & 20 & 10 & 99.59 \\
\hline 6 & 1 & 20 & 10 & 99.84 \\
\hline 10 & 1 & 20 & 10 & 99.90 \\
\hline 14 & 1 & 20 & 10 & 99.94 \\
\hline 2 & 2 & 5 & 5 & 98.71 \\
\hline 6 & 2 & 5 & 5 & 99.50 \\
\hline 10 & 2 & 5 & 5 & 99.70 \\
\hline 14 & 2 & 5 & 5 & 99.62 \\
\hline 2 & 2 & 10 & 5 & 98.94 \\
\hline 6 & 2 & 10 & 5 & 99.67 \\
\hline 10 & 2 & 10 & 5 & 99.74 \\
\hline 14 & 2 & 10 & 5 & 99.68 \\
\hline 2 & 2 & 15 & 5 & 99.01 \\
\hline 6 & 2 & 15 & 5 & 99.65 \\
\hline 10 & 2 & 15 & 5 & 99.79 \\
\hline 14 & 2 & 15 & 5 & 99.81 \\
\hline 2 & 2 & 20 & 5 & 99.21 \\
\hline 6 & 2 & 20 & 5 & 99.54 \\
\hline 10 & 2 & 20 & 5 & 99.83 \\
\hline 14 & 2 & 20 & 5 & 99.88 \\
\hline 2 & 2 & 5 & 7.5 & 98.40 \\
\hline 6 & 2 & 5 & 7.5 & 99.57 \\
\hline 10 & 2 & 5 & 7.5 & 99.82 \\
\hline 14 & 2 & 5 & 7.5 & 99.90 \\
\hline 2 & 2 & 10 & 7.5 & 99.3 \\
\hline 6 & 2 & 10 & 7.5 & 99.70 \\
\hline 10 & 2 & 10 & 7.5 & 99.84 \\
\hline 14 & 2 & 10 & 7.5 & 99.84 \\
\hline 2 & 2 & 15 & 7.5 & 99.42 \\
\hline 6 & 2 & 15 & 7.5 & 99.75 \\
\hline 10 & 2 & 15 & 7.5 & 99.85 \\
\hline 14 & 2 & 15 & 7.5 & 99.87 \\
\hline
\end{tabular}




\begin{tabular}{|c|c|c|c|c|}
\hline 2 & 2 & 20 & 7.5 & 99.50 \\
\hline 6 & 2 & 20 & 7.5 & 99.82 \\
\hline 10 & 2 & 20 & 7.5 & 99.88 \\
\hline 14 & 2 & 20 & 7.5 & 99.90 \\
\hline 2 & 2 & 5 & 10 & 98.84 \\
\hline 6 & 2 & 5 & 10 & 99.63 \\
\hline 10 & 2 & 5 & 10 & 99.75 \\
\hline 14 & 2 & 5 & 10 & 99.73 \\
\hline 2 & 2 & 10 & 10 & 99.08 \\
\hline 6 & 2 & 10 & 10 & 99.76 \\
\hline 10 & 2 & 10 & 10 & 99.79 \\
\hline 14 & 2 & 10 & 10 & 99.66 \\
\hline 2 & 2 & 15 & 10 & 99.60 \\
\hline 6 & 2 & 15 & 10 & 99.81 \\
\hline 10 & 2 & 15 & 10 & 99.54 \\
\hline 14 & 2 & 15 & 10 & 99.92 \\
\hline 2 & 2 & 20 & 10 & 99.31 \\
\hline 6 & 2 & 20 & 10 & 99.24 \\
\hline 10 & 2 & 20 & 10 & 99.89 \\
\hline 14 & 2 & 20 & 10 & 99.89 \\
\hline 8.5 & 1 & 5 & 7.5 & 99.53 \\
\hline 8.5 & 1 & 5 & 7.5 & 99.26 \\
\hline 8.5 & 1 & 5 & 7.5 & 99.33 \\
\hline 8.5 & 1 & 12.5 & 7.5 & 98.83 \\
\hline 8.5 & 1 & 12.5 & 7.5 & 99.22 \\
\hline 8.5 & 1 & 12.5 & 7.5 & 99.71 \\
\hline 8.5 & 1 & 20 & 7.5 & 98.79 \\
\hline 8.5 & 1 & 20 & 7.5 & 99.71 \\
\hline 8.5 & 1 & 20 & 7.5 & 96.09 \\
\hline 8.5 & 2 & 5 & 7.5 & 95.17 \\
\hline 8.5 & 2 & 5 & 7.5 & 99.69 \\
\hline 8.5 & 2 & 5 & 7.5 & 95.84 \\
\hline 8.5 & 2 & 12.5 & 7.5 & 99.93 \\
\hline 8.5 & 2 & 12.5 & 7.5 & 99.82 \\
\hline 8.5 & 2 & 12.5 & 7.5 & 99.11 \\
\hline 8.5 & 2 & 20 & 7.5 & 99.53 \\
\hline 8.5 & 2 & 20 & 7.5 & 98.67 \\
\hline 8.5 & 2 & 20 & 7.5 & 99.86 \\
\hline
\end{tabular}




\section{E-1 ANOVA RESULTS}

ANOVA was conducted step-wise, first including all parameters, and then removing those which did not have a significant effect.

\section{Step 1:}

SUMMARY

OUTPUT

\begin{tabular}{lr}
\multicolumn{2}{c}{ Regression Statistics } \\
\hline Multiple R & 0.733027154 \\
R Square & 0.537328809 \\
Adjusted R Square & 0.516991614 \\
Standard Error & 0.001975243 \\
Observations & 96 \\
\hline
\end{tabular}

$\begin{array}{ll}\text { Pooled } & \\ \text { variance } & 0.00017175\end{array}$

ANOVA

\begin{tabular}{|c|c|c|c|c|c|}
\hline & $d f$ & SS & $M S$ & $F$ & $\begin{array}{c}\text { Significance } \\
F \\
\end{array}$ \\
\hline Regression & 4 & 0.000412335 & 0.000103084 & 26.42098891 & $1.49816 \mathrm{E}-14$ \\
\hline Residual & 91 & 0.000355044 & $3.90158 \mathrm{E}-06$ & & \\
\hline \multirow[t]{2}{*}{ Total } & 95 & 0.000767379 & & & \\
\hline & Coefficients & Lower 95\% & Upper 95\% & & \\
\hline Intercept & 0.995840448 & 0.995597732 & 0.996083164 & & \\
\hline concentration & 0.000359238 & 0.000116522 & 0.000601954 & & \\
\hline mass & $9.85951 \mathrm{E}-05$ & -0.000144121 & 0.000341311 & & \\
\hline time & 8.28747E-05 & -0.000159841 & 0.000325591 & & \\
\hline batch & 0.002350927 & $\underline{-0.002593643}$ & $\underline{-0.002108211}$ & & \\
\hline
\end{tabular}




\section{Step 2:}

SUMMARY OUTPUT

\begin{tabular}{|c|c|c|c|c|c|c|}
\hline \multicolumn{2}{|c|}{ Regression Statistics } & & & & \multirow[t]{2}{*}{$\begin{array}{l}\text { Pooled } \\
\text { variance }\end{array}$} & \multirow[t]{2}{*}{$\begin{array}{r}0.00017 \\
2\end{array}$} \\
\hline Multiple R & 0.704092 & & & & & \\
\hline R Square & 0.495745 & & & & & \\
\hline Adjusted R & & & & & & \\
\hline Square & 0.484901 & & & & & \\
\hline Standard Error & 0.00204 & & & & & \\
\hline Observations & 96 & & & & & \\
\hline \multicolumn{7}{|l|}{ ANOVA } \\
\hline & $d f$ & SS & $M S$ & $F$ & Significance $F$ & \\
\hline & & & & \multicolumn{2}{|l|}{45.7153} & \\
\hline \multirow{2}{*}{ Regression } & 2 & 0.00038 & 0.00019 & 2 & $1.49 \mathrm{E}-14$ & \\
\hline & & 0.00038 & & & & \\
\hline \multirow[t]{2}{*}{ Residual } & 93 & 7 & $4.16 \mathrm{E}-06$ & & & \\
\hline & & 0.00076 & & & & \\
\hline \multirow[t]{4}{*}{ Total } & 95 & 7 & & & & \\
\hline & Coefficient & Lower & Upper & & & \\
\hline & 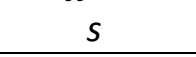 & $95 \%$ & $95 \%$ & & & \\
\hline & & 0.99745 & 0.99793 & & & \\
\hline \multirow[t]{2}{*}{ Intercept } & 0.997694 & 2 & 7 & & & \\
\hline & & 0.00011 & 0.00060 & & & \\
\hline concentration & 0.000359 & 7 & 2 & & & \\
\hline batch & -0.00235 & -0.00259 & -0.00211 & & & \\
\hline
\end{tabular}




\section{APPENDIX F: OTHER PHARMACEUTICAL RESULTS}

\begin{tabular}{|c|c|c|c|c|}
\hline Pharmaceutical & $\begin{array}{l}\text { Untreated } \\
\text { deionized } \\
\text { water }(\mathrm{ng} / \mathrm{L})\end{array}$ & $\begin{array}{l}\text { Treated } \\
\text { deionized water } \\
(\mathrm{ng} / \mathrm{L})\end{array}$ & $\begin{array}{l}\text { Untreated } \\
\text { wastewater } \\
(\mathrm{ng} / \mathrm{L})\end{array}$ & $\begin{array}{l}\text { Treated } \\
\text { wastewater } \\
(\mathrm{ng} / \mathrm{L})\end{array}$ \\
\hline azithromycin & 2.63 & 29.2 & 318 & 226 \\
\hline carbadox & 89.4 & 42.2 & 61.9 & 66 \\
\hline cefotaxime & $\mathrm{NQ}^{*}$ & 27.9 & 78.6 & 172 \\
\hline ciprofloxacin & 6.89 & ND & 3080 & ND \\
\hline clarithromycin & 10.8 & 27.9 & ND & 316 \\
\hline clinafloxacin & $\mathrm{ND}^{* *}$ & 20.6 & 2.65 & ND \\
\hline dehydronifedipine & ND & ND & 279 & 2.96 \\
\hline diltiazem & 31.9 & 11.6 & 7.5 & 163 \\
\hline digoxin & ND & ND & ND & 6.6 \\
\hline digoxigenin & ND & ND & 86.5 & ND \\
\hline erythromycin- $\mathrm{H} 20$ & 12.4 & 15.9 & ND & 92.7 \\
\hline flumequine & ND & ND & 82.4 & ND \\
\hline fluoxetine & 40.5 & ND & 10.1 & 2.3 \\
\hline miconazole & 10.7 & ND & ND & ND \\
\hline norfloxacin & ND & ND & ND & ND \\
\hline norgestimate & ND & ND & ND & ND \\
\hline ormetoprim & ND & ND & 445 & ND \\
\hline oxolinic acid & ND & ND & ND & ND \\
\hline penicillin $\mathrm{G}$ & ND & ND & 10.1 & 10.9 \\
\hline penicillin V & ND & ND & ND & ND \\
\hline roxithromycin & 3.49 & 7.12 & 426 & 35.6 \\
\hline sarafloxacin & ND & ND & ND & ND \\
\hline sulfachloropyridazine & ND & 4.63 & ND & $\mathrm{NQ}$ \\
\hline sulfadimethoxine & 76.1 & 8.95 & NQ & NQ \\
\hline sulfamerazine & ND & ND & ND & $\mathrm{NQ}$ \\
\hline sulfamethazine & 266 & 222 & 134 & 222 \\
\hline sulfamethoxazole & ND & ND & 175 & 616 \\
\hline thiabendazole & ND & ND & 8.66 & 9.26 \\
\hline trimethoprim & 46.7 & 7.07 & 113 & 53.4 \\
\hline tylosin & 18.4 & 24.2 & 3060 & 303 \\
\hline virginiamycin M1 & ND & 218 & ND & 593 \\
\hline 1,7-dimethylxanthine & ND & ND & 77.5 & ND \\
\hline
\end{tabular}




\section{APPENDIX G: DISTRIBUTION OF HYDROLYSIS PRODUCTS FOR LEAD, COPPER, ZINC, AND NICKEL (BAES AND MESMER, 1976)}
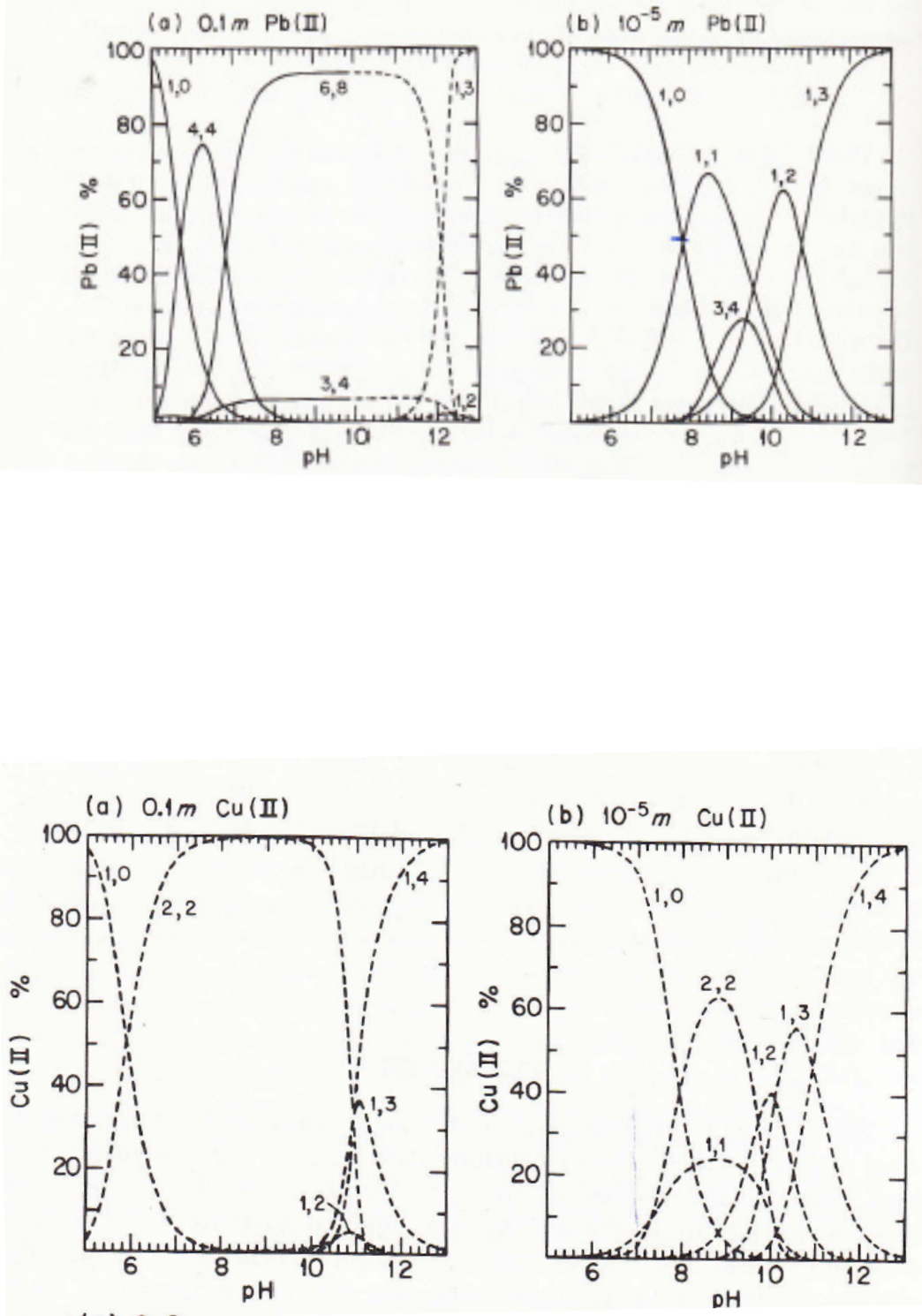

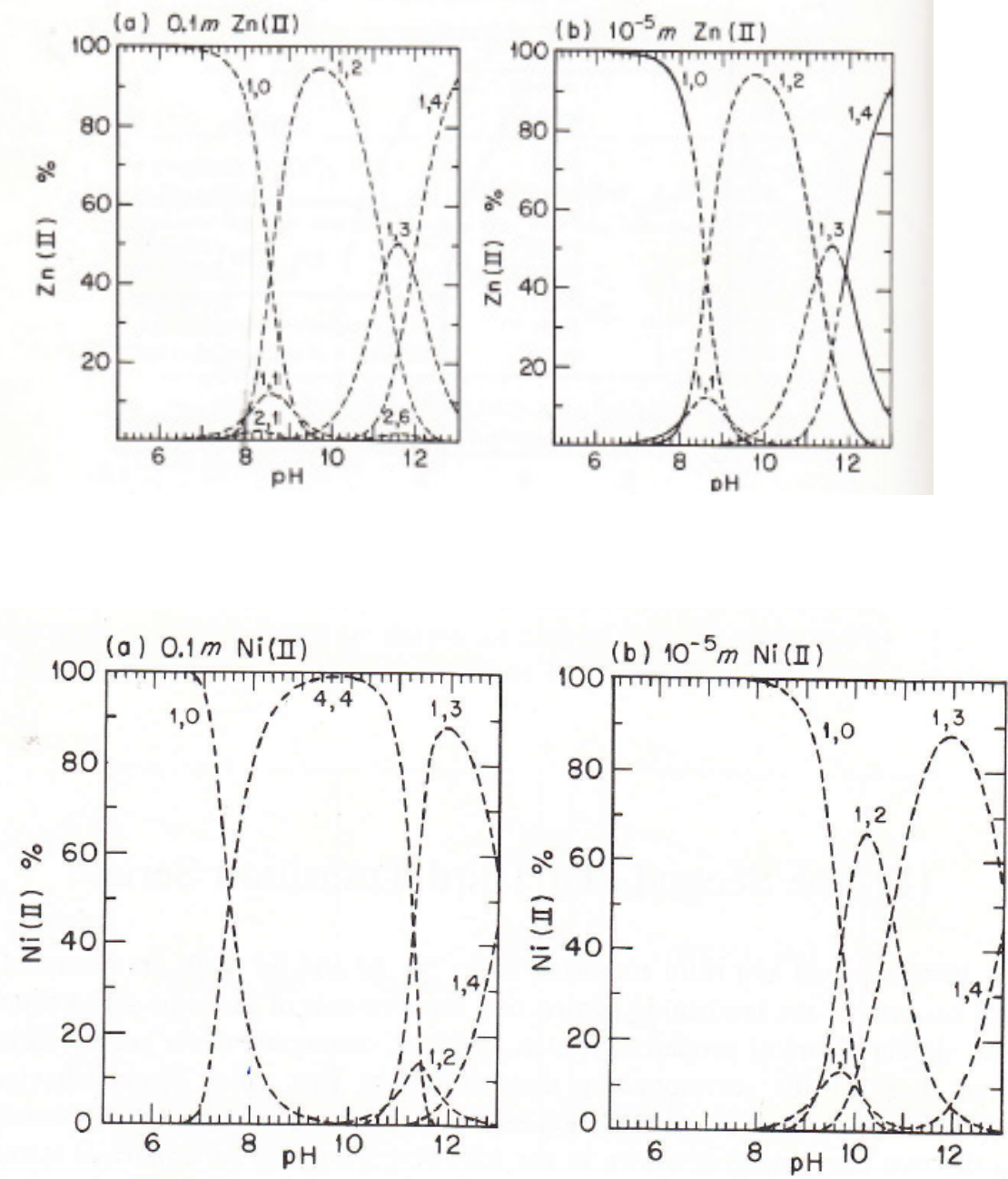


\section{APPENDIX H: BALLASTED FLOCCULATION ANOVA}

ANOVA was conducted step-wise, first including all parameters, and then removing those which did not have a significant effect.

\section{Step 1: \\ SUMMARY \\ OUTPUT}

\begin{tabular}{|c|c|c|c|c|c|}
\hline \multicolumn{2}{|c|}{ Regression Statistics } & & & & \\
\hline Multiple R & 0.9351 & & & & \\
\hline R Square & 0.8744 & & & & \\
\hline Adjusted R Square & 0.7309 & & & & \\
\hline Standard Error & 0.6936 & & & & \\
\hline Observations & 16.0000 & & & & \\
\hline \multicolumn{6}{|l|}{ ANOVA } \\
\hline & $d f$ & SS & $M S$ & $F$ & $\begin{array}{c}\text { Significance } \\
F\end{array}$ \\
\hline Regression & 8.0000 & 23.4437 & 2.9305 & 6.0917 & 0.0139 \\
\hline Residual & 7.0000 & 3.3674 & 0.4811 & & \\
\hline Total & 15.0000 & 26.8111 & & & \\
\hline
\end{tabular}

\begin{tabular}{|c|c|c|c|c|c|c|}
\hline & Coefficients & Standard Error & t Stat & $\begin{array}{c}P- \\
\text { value }\end{array}$ & Lower 95\% & $\begin{array}{c}\text { Upper } \\
95 \%\end{array}$ \\
\hline Intercept & 9.5464 & 2.7307 & 3.4960 & 0.0100 & 3.0894 & 16.0035 \\
\hline X Variable 1 & -0.4724 & 0.1161 & 4.0685 & 0.0048 & -0.7470 & -0.1978 \\
\hline X Variable 2 & 0.0322 & 0.0437 & 0.7365 & 0.4853 & -0.0712 & 0.1356 \\
\hline X Variable 3 & 0.0128 & 0.0087 & 1.4669 & 0.1858 & -0.0079 & 0.0335 \\
\hline X Variable 4 & -0.0990 & 0.1841 & 0.5378 & 0.6074 & -0.5342 & 0.3362 \\
\hline X Variable 5 & -0.0059 & 0.0023 & 2.5246 & 0.0395 & -0.0114 & -0.0004 \\
\hline X Variable 6 & -0.4804 & 0.1908 & 2.5175 & 0.0400 & -0.9317 & -0.0292 \\
\hline X Variable 7 & -0.1419 & 0.1554 & - & 0.3915 & -0.5092 & 0.2255 \\
\hline X Variable 8 & -0.0296 & 0.1280 & 0.2311 & 0.8239 & -0.3321 & 0.2730 \\
\hline
\end{tabular}




\section{Step 2:}

SUMMARY OUTPUT

\begin{tabular}{lr}
\hline \multicolumn{2}{c}{ Regression Statistics } \\
\hline Multiple R & 0.896136 \\
R Square & 0.80306 \\
Adjusted R & \\
Square & 0.753825 \\
Standard Error & 0.663336 \\
Observations & 16 \\
\hline
\end{tabular}

ANOVA

\begin{tabular}{|c|c|c|c|c|c|c|}
\hline & $d f$ & SS & MS & $F$ & $\begin{array}{c}\text { Significanc } \\
e F\end{array}$ & \\
\hline & & & 7.17697 & 16.3107 & & \\
\hline \multirow[t]{2}{*}{ Regression } & 3 & 21.53091 & 1 & 4 & 0.000156 & \\
\hline & & & 0.44001 & & & \\
\hline Residual & 12 & 5.280181 & 5 & & & \\
\hline \multirow[t]{4}{*}{ Total } & 15 & 26.81109 & & & & \\
\hline & Coefficient & Standard & & & & Upper \\
\hline & $s$ & Error & t Stat & P-value & Lower 95\% & $95 \%$ \\
\hline & & & 9.21782 & & & 10.0006 \\
\hline Intercept & 8.08875 & 0.877511 & 9 & 8.57E-07 & 6.176817 & 8 \\
\hline \multirow{2}{*}{ X Variable 1} & -0.39225 & 0.066334 & -5.91329 & 7.11E-05 & -0.53678 & -0.24772 \\
\hline & & & & 0.01801 & & \\
\hline X Variable 2 & -0.00605 & 0.002211 & -2.73742 & 7 & -0.01087 & -0.00124 \\
\hline X Variable 3 & -0.42188 & 0.165834 & -2.54396 & 0.02575 & -0.7832 & -0.06055 \\
\hline
\end{tabular}

